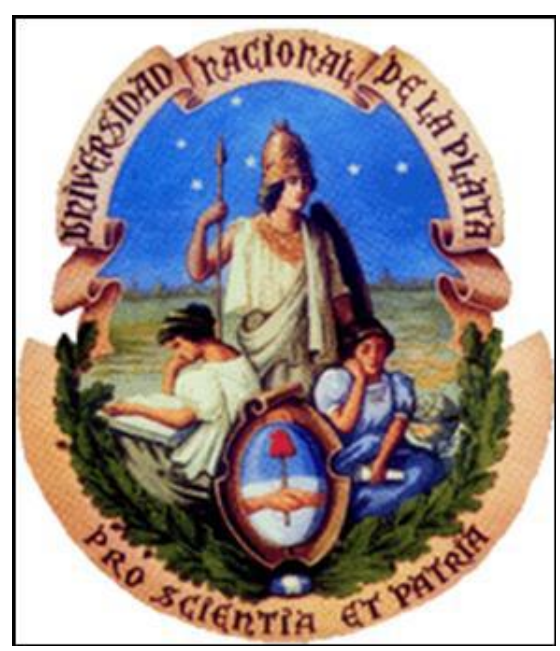

Universidad Nacional de La Plata

Facultad de Ciencias Exactas

Departamento de Química.

Trabajo de tesis doctoral:

\title{
Estudio Espectroelectroquímico de Compuestos de Coordinación Para Aplicaciones Catalíticas.
}

Autor: Lic. Jackeline Muñoz Zúñiga

Director: Dr. Reynaldo O. Lezna

Codirector: Dr. Ezequiel Wolcan

2012 
El presente trabajo de tesis, para optar por el título de Doctor de la Facultad de Ciencias Exactas de la Universidad Nacional de La Plata, fue realizado en el Instituto de Investigaciones Fisicoquímicas Teóricas y Aplicadas (INIFTADepartamento de Química) de la Facultad de Ciencias Exactas, Universidad Nacional de La Plata.

I $\mathrm{N}$ I $\mathrm{F}$ T A

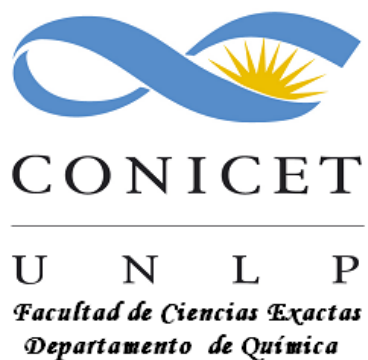



A mi madre y a Luza, por el valor mostrado para salir adelante y por su amor. 


\section{Agradecimientos}

La presente Tesis Doctoral, es el producto de varios años de trabajo donde culmina una etapa de mi vida. En la elaboración de la misma participaron directa e indirectamente personas e instituciones a quienes quiero agradecer.

A la Facultad de Ciencias Exactas y a la Universidad Nacional de la Plata, por brindar educación pública y gratuita de excelencia.

Al Dr. Reynaldo Lezna por su dirección, su enseñanza sobre la ciencia, por darme la oportunidad de formar parte del grupo de investigación a su cargo y por permitir desarrollar mi trabajo de tesis.

A la Dra. Norma de Tacconi por su permanente colaboración y sus valiosos aportes en la realización de este proyecto.

A mi codirector Dr. Ezequiel Wolcan por su buena disposición y colaboración.

A mis jurados de tesis por dedicar su valioso tiempo a la lectura de la misma, por sus sugerencias y por sus importantes aportes.

A mis compañeros del cuarto piso (investigadores y becarios) por hacer más placentera mi estadía allí. 
A Silvina, Mavi, Mariela, Yeimi y Juliana por su apoyo constante, confidencialidad y sensibilidad ante cada situación. Pero principalmente por su amistad.

Quiero agradecer a mi familia, porque a pesar de la distancia, siempre estuvieron alentándome y nunca dejaron de creer en mí, especialmente a Luza y Yony por su apoyo incondicional y su marcado interés en mis proyectos.

A mi madrecita, quien con sus comentarios y risas alegra siempre cualquier momento tenso y me llena de fuerza para continuar.

A mi papi, por tratar demostrar que la prudencia es una buena aliada y por su constante lucha frente a la vida.

A mis abuelos Clelia y Alfredo, por haberme dado las raíces y el ejemplo de vida.

A mis hermanos, Ximena, Yony y David quienes junto a los angelitos que llegaron a mi vida, Isabel y Gabriela, son y seguirán siendo mi motivo de inspiración.

A las anfetaminas de Unicauca, aunque un poco dispersas, siempre juntas de corazón.

A Patricia Rodriguez, Vicky Ortiz, Aleyda Camacho, Ceyda Benavides, Rosa Macias, Norita Rivera, Mayra Rubio, Rocio Chilito, Yolima Ledezma, David Lozada, Alexander Garcés, Julián Sánchez, Oscar Bermeo, Julián Garcés, Luis Alexander Navia, Diego Barbosa, Mario Guzmán y mis demás amigos de Colombia, por su apoyo, cariño y por alegrarse con mis logros.

A Carolina Mendoza, Nancy Lovera, Adriana Trujillo, Selma Martinez, Mavi Humana, mis compañeras de vida, las que iban siendo mi familia y cumplieron muy bien su rol. Gracias chicas por el aguante y por soportar tantas horas a mi lado. 
A estas personas con corazón colombiano que me crucé un día en calles argentinas y sentí que las conocía de toda la vida, por ser mi soporte, mi familia, y mis amigos.

A Cecilia y su familia, por depositar tanta confianza en mí y así facilitar mi estadía en la Argentina

A Silvina, por su compañerismo, su ayuda, apoyo. Por las interminables charlas y risas... pero principalmente por su amistad.

A Mariela (y su familia), por abrirme las puertas de su casa, por los asados, por la caminata y fundamentalmente por su cariño sincero.

A Adriana Rodriguez, Federico Degrange, Dafne Amaya, Carla Berghoff, Ezequiel López, Roberto Urcuyo y Natalia Fagali por las lindas charlas, las risas y fundamentalmente por su amistad.

A los chicos del taller cultural por la buena onda siempre, por su alegría y compañerismo.

A Ofelia, por su profesionalismo, responsabilidad y sobre todo por abrirme las puertas de su casa y brindarme su amistad.

A Guille, por todo su cariño.

A Mónica Moreno, Cristina Ruales, Carmen Mosquera, Cristian Buendía, Vaneza Lorett, Oscar Amelines, Roberto Castillo y a todos quienes me ayudaron en la búsqueda bibliográfica.

A la gente de Ciencia ConCiencia, con quienes comparto sueños probablemente no tan lejanos. 
A todo el personal del INIFTA por la buena predisposición y ayuda.

Finalmente, a la ANPCyT y al CONICET por otorgarme las becas de postgrado para realizar este trabajo de Tesis. 



\section{Resumen}

Los estudios con compuestos de coordinación han contribuido con aportes relevantes a la catálisis. Los catalizadores de este tipo más estudiados en las últimas cuatro décadas incluyen complejos de Re (I), Ru (II), Os (II), Ir (III). Investigaciones catalíticas con complejos de coordinación comprenden sistemas tanto homogéneos como heterogéneos e involucran reacciones de reducción de $\mathrm{CO}_{2}$, reacciones de aminación de azidas orgánicas con ligandos coordinados, degradación de contaminantes ambientales, etc.

El interés por el estudio de complejos de metales de transición como catalizadores ha venido creciendo en los últimos años, Lehn et al., fueron los primeros en reportar la reducción fotocatalítica de $\mathrm{CO}_{2}$ por un complejo de $\mathrm{Re}$, empleando complejos de la forma $\operatorname{Re}(\mathrm{L})(\mathrm{CO})_{3} \mathrm{X}$ (donde $\mathrm{L}: 2,2^{\prime}$ - bpy o 1,10-phen y $\mathrm{X}$ : $\mathrm{Cl}$ o $\mathrm{Br}$ ) utilizando TEA (trietanolamina) como reductor de sacrificio.

Otro ejemplo analizado por Lehn y colaboradores es el estudio de tres sistemas en la reducción fotocatalítica de $\mathrm{CO}_{2}$. El primer sistema contiene $\left[\mathrm{Ru}(\mathrm{bpy})_{3}\right]^{2+}$ y $\left[\mathrm{Co}(\mathrm{bpy})_{3}\right]^{2+}$ o iones Co (II) (como mediador electrónico y como catalizador de las reducciones de $\mathrm{H}_{2} \mathrm{O}$ y $\mathrm{CO}_{2}$ ), dando lugar a la generación de $\mathrm{H}_{2}$ y $\mathrm{CO}$. El segundo sistema, basado en el complejo $f a c-\left[\operatorname{Re}(b p y)(C O){ }_{3} \mathrm{Cl}\right]$ ha producido altos rendimientos de $\mathrm{CO}$. El tercer sistema, involucra complejos de $\mathrm{Ru}$ y produjo cantidades catalíticas, pequeñas, de formiato.

En este trabajo se describe el estudio espectroelectroquímico de complejos de coordinación con el objetivo de relacionar sus propiedades electroquímicas y 
espectroscópicas con su comportamiento fotoquímico y fotofísico. Fueron objeto de esta investigación compuestos de coordinación de $\mathrm{Ru}, \mathrm{Re}$ y grupos piracínicos (tatpp). La potencialidad de estos compuestos como catalizadores de procesos de eliminación de contaminantes, e.g. $\mathrm{CO}_{2}$, como agentes de clivaje de la molécula de ADN y para la generación de hidrógeno solar, fue investigada con técnicas electroquímicas y fotoasistidas.

El primer capítulo introduce los complejos de coordinación estudiados analizando sus estructuras en comparación con las de sistemas fotosintéticos artificiales. En el segundo capítulo se desarrolla la teoría de los diferentes temas a tratar a lo largo de la tesis, además de la descripción de las técnicas utilizadas.

En el tercer y cuarto capítulo se detalla el estudio espectroelectroquímico de los complejos de $\mathrm{Ru}\left[(\text { phen })_{2} \mathrm{Ru}(\text { tatpp } \alpha) \mathrm{Ru}(\text { phen })_{2}\right]^{4+}\left(1^{4+}\right)$ y $\left[(\text { bpy })_{2} R u(\text { tatpp })\right]^{2+}$ $\left(3^{2+}\right)$ respectivamente. Ambos complejos poseen el grupo tatpp como ligando central y varían en el tipo y número de cromóforos presentes en cada especie.

Los estudios electroquímicos se realizaron utilizando técnicas de voltamperometría de contínua (CV) y de alterna (ACV) con el fin de identificar los perfiles de electroreducción del complejo a analizar y la electrooxidación del $\mathrm{Ru}$ en cada sistema.

Los espectros UV-Vis tanto del complejo como de su ligando central (en este último caso con exceso de iones $\mathrm{Zn}$ (II) para lograr su solubilización) se obtuvieron de manera convencional.

La identificación de las especies reducidas en los estudios espectroelectroquímicos se realizó con la ayuda de titulaciones químicas con cobaltoceno, monitoreada a través de espectros de absorción convencionales a diferentes tiempos. Las medidas espectroelectroquímicas comprendieron principalmente dos métodos complementarios, espectros integrales de absorción UV-Vis in-situ a potenciales estacionarios con resolución temporal (con un OMA capítulo 2 ) y reflectancia diferencial $(\delta \mathrm{R} / \mathrm{R})$ a longitudes de onda fijas. 
En el quinto capítulo se realizó el estudio espectroelectroquímico de polímeros de renio $\mathrm{CF}_{3} \mathrm{SO}_{3}\left[\mathrm{Re}(\mathrm{CO})_{3}\left(\mathrm{NO}_{2}\right.\right.$-phen)-P4VPy] $(\boldsymbol{P V}-5 \mathrm{~N})$ y $\mathrm{CF}_{3} \mathrm{SO}_{3}$ $\left[\mathrm{Re}(\mathrm{CO})_{3}\right.$ (tmphen)-P4VPy] (PV-TM) y de sus complejos relacionados $\left[\mathrm{CF}_{3} \mathrm{SO}_{3}\right.$ $\operatorname{Re}^{\prime}(\mathrm{CO})_{3}\left(\mathrm{NO}_{2}\right.$-phen)] (TF-5N), [ $\mathrm{CF}_{3} \mathrm{SO}_{3} \operatorname{Re}^{\prime}(\mathrm{CO})_{3}($ tmphen $\left.)\right]$ (TF-TM),

Para identificar las especies generadas electroquímicamente asociadas a las ondas medidas por ACV, se tomaron, en este caso (complejos de Re), espectros (integrales) in-situ, utilizando un OMA (capítulo 2) que permitieron identificar las fracciones reducidas en cada complejo/polímero.

La utilización de los métodos espectroelectroquímicos ha facilitado significativamente la deducción del mecanismo de reducción para el complejo $3^{2+} y$ la identificación de reacciones colaterales en $\mathbf{1}^{4+}$. 


\section{"Estudio Espectroelectroquímico de Compuestos de Coordinación para Aplicaciones Catalíticas".}

Dedicatoria.

Agradecimiento.

Resumen.

\section{Índice de contenido}

Acrónimos.

Capítulo 1. Introducción

pág

$\begin{array}{lll}1.1 & \text { Antecedentes } & 4\end{array}$

$\begin{array}{lll}1.2 & \text { Fotosíntesis artificial } & 7\end{array}$

1.2.1 Ensamblaje molecular para fotosíntesis artificial 10

1.3 Compuestos de coordinación de Ru para reacciones de interés $\begin{array}{ll}\text { ambiental } & 14\end{array}$

1.3.1 ¿Cómo actúan los complejos estudiados? 16

1.4 Otros diseños y usos de complejos con aplicaciones similares $\quad 19$

1.5 Referencias 21 


\section{Capítulo 2. Materiales y métodos.}

2.1 Parte experimental $\quad 5$

$\begin{array}{lll}2.2 & \text { Medidas electroquímicas } & 7\end{array}$

2.2.1 La doble capa eléctrica $\quad 8$

2.2.2 Cinética electroquímica 9

2.2.3 Determinación de la cinética de electrodos 14

2.2.4 Pulsos de potencial $\quad 15$

$\begin{array}{lll}2.2 .5 & \text { Voltamperometría } & 18\end{array}$

2.2.5.1 Voltamperometría de corriente continua - DCV 18

2.2.5.2 Voltamperometría de corriente alterna - ACV 24

2.3 Medidas Ópticas In-Situ 29

2.3.1 Espectroscopía UV-vis - (OTTLEs) 31

2.3.2 Reflectancia Total Atenuada (ATR) 32

2.3.3 Espectroscopía de reflectancia modulada especular 35

2.3.3.1 Reflectancia externa $\quad 35$

2.3.4 Espectroscopía de reflectancia para especies adsorbidas $\begin{array}{ll}\text { sobre la superficie del electrodo } & 37\end{array}$

2.3.5 Espectroscopía de reflectancia de especies en solución $\quad 40$

2.3.6 Medidas diferenciales $\quad 41$

2.3.7 Medidas integrales 44 
Capítulo 3. Electroquímica y espectroelectroquímica del complejo $\left[(\text { phen })_{2} \operatorname{Ru}(\text { tatpp } \alpha) \operatorname{Ru}(\text { phen })_{2}\right]^{4+}\left(1^{4+}\right)$

3.1 Introducción

3.2 Electroquímica de $\left[(\text { phen })_{2} R u(\operatorname{tatpp} \alpha) \operatorname{Ru}(\text { phen })_{2}\right]^{4+}\left(1^{4+}\right)$

3.3 Espectro electrónico de $\mathbf{1}^{4+}$

3.4 Características de la forma oxidada y reducida de $\mathbf{1}^{4+}$

3.5 Espectroelectroquímica del complejo

$\left[(\text { phen })_{2} \mathrm{Ru}(\text { tatpp } \alpha) \mathrm{Ru}(\text { phen })_{2}\right]^{4+}\left(\mathbf{1}^{4+}\right)$

3.6 Discusión

3.7 Conclusiones

3.8 Referencias

Capítulo 4. Reducción reversible multielectrónica del 9,11,20,22tetraaza-tetrapiridopentaceno (tatpp) como ligando axial en el complejo $\left[(\text { bpy })_{2} \mathrm{Ru}(\right.$ tatpp) $] \mathrm{Cl}_{2}$.

4.1 Introducción 4

$\begin{array}{lll}4.2 & \text { Parte experimental } & 7\end{array}$

4.3 Electroquímica de $\left[(\text { bpy })_{2} R u(\text { tatpp })\right]^{2+}\left(3^{2+}\right)$ y del aducto Zn-tatpp 9 
4.4 Espectro de absorción para $3^{2+}$ y de las especies relacionadas formadas químicamente

4.5 Espectroelectroquímica del complejo $\left[(\text { bpy })_{2} R u(\text { tatpp })\right]^{2+}\left(3^{+2}\right)$ y del aducto Zn-tatpp

$\begin{array}{lll}4.6 & \text { Discusión } & 22\end{array}$

4.7 Mecanismo de electroreducción 24

$\begin{array}{llr}4.8 & \text { Conclusiones } & 29\end{array}$

4.9 Referencias 31

Capítulo 5. Espectroelectroquímica de radicales aniones generados en la electroreducción de $-\left[\operatorname{Re}^{\prime}(\mathrm{CO})_{3}(5-N i t r o-1,10-f e n a n t r o l i n a)\right]^{+}$y $\left[\operatorname{Re}^{\prime}(\mathrm{CO})_{3}(3,4,7,8-t e t r a m e t i l-1,10-\text { fenantrolina }]^{+}\right.$en polímeros de poli- 4vinilpiridina.

5.1 Medidas electroquímicas

5.2 Espectroelectroquímica de los complejos TF-5N, TF-TM, PV-5N, PV-TM

5.3 Discusión

5.4 Conclusiones

5.5 Referencias

Trabajos publicados y presentaciones a congresos en el marco del trabajo de tesis. 


\section{Listado de Acrónimos}

\begin{tabular}{|c|c|c|}
\hline $\mathrm{ACV}$ & : & Voltamperometría de corriente alterna. \\
\hline DCV & : & Voltamperometría de corriente contínua. \\
\hline CV & : & Voltamperometría cíclica. \\
\hline AC & : & Corriente alterna. \\
\hline DC & : & Corriente continua. \\
\hline $\mathrm{z}$ & : & Número de electrones. \\
\hline $\mathrm{HOMO}$ & : & Orbital molecular ocupado de más alta energía. \\
\hline LUMO & : & Orbital molecular desocupado de más baja energía. \\
\hline LC & : & Transferencia de carga centrada en el ligando. \\
\hline MLCT & : & Transferencia de carga metal - ligando. \\
\hline Phen & : & Fenantrolina. \\
\hline Bpy & : & Bipiridina. \\
\hline Tatpp $\alpha$ & : & $\begin{array}{l}\text { Ligando puente central (doblado) de complejos } 1^{4+} y \\
4^{2+} \text {. }\end{array}$ \\
\hline Tatpp $\beta$ & : & Ligando puente central (lineal) de complejos $2^{4+}$ y $3^{2+}$ \\
\hline $\mathrm{MeCN}$ & : & Acetonitrilo. \\
\hline TBAPF $_{6}$ & : & Hexafluorofosfato de tetrabutilamonio. \\
\hline LIA & : & Lock-in amplifier. \\
\hline IRS & : & Espectroscopía de reflexión interna. \\
\hline ATR & : & Reflectancia total atenuada. \\
\hline$\varepsilon$ & : & Coeficiente de absortividad molar. \\
\hline$I$ & : & Corriente. \\
\hline$j$ & : & Densidad de corriente. \\
\hline$j_{0}$ & : & Densidad de corriente de intercambio \\
\hline$v$ & : & Velocidad de barrido de potencial. \\
\hline$t$ & : & Tiempo. \\
\hline
\end{tabular}




\begin{tabular}{|c|c|c|}
\hline$I_{d}$ & : & Corriente de difusión. \\
\hline$D_{0}$ & : & Coeficiente de difusión de la especie $O$. \\
\hline$n$ & : & Índice de refracción real. \\
\hline$\hat{n}$ & : & Forma compleja del índice de refracción. \\
\hline $\mathrm{C}^{*} \mathrm{O}$ & : & Concentración de la especie $O$ en solución. \\
\hline $\mathrm{Co}_{\mathrm{O}}$ & : & Concentración de la especie O en la interfase. \\
\hline$E_{i}$ & : & Potencial inicial. \\
\hline$E_{\lambda}$ & : & Potencial de inversión del barrido de potencial en CV. \\
\hline$E^{o}$ & : & Potencial en condiciones estándar. \\
\hline$F$ & : & Constante de Faraday. \\
\hline $\mathrm{T}$ & : & Temperatura, ${ }^{\circ} \mathrm{K}$. \\
\hline$u(t)$ & : & Potencial sinusoidal \\
\hline$\eta$ & : & Sobrepotencial. \\
\hline$\alpha_{A}$ & : & $\begin{array}{l}\text { Coeficiente de transferencia de la hemireacción } \\
\text { anódica. }\end{array}$ \\
\hline$\alpha_{C}$ & : & $\begin{array}{l}\text { Coeficiente de transferencia de la hemireacción } \\
\text { catódica. }\end{array}$ \\
\hline$\hat{\in}_{n}$ & : & Constante dieléctrica compleja de la fase $n$. \\
\hline$k$ & : & Constante de velocidad de las especies electroactivas. \\
\hline $\mathrm{R}_{\mathrm{s}}$ & : & Resistencia óhmica de la solución. \\
\hline $\mathrm{R}_{\mathrm{ct}}$ & : & Resistencia de la transferencia de carga. \\
\hline$Z_{w}$ & : & Impedancia de Warburg. \\
\hline $\mathrm{C}_{\mathrm{d}}$ & : & Doble capa - capacitancia. \\
\hline UPD & : & Deposición a subpotenciales. \\
\hline ET & : & Transferencia electrónica. \\
\hline
\end{tabular}


1. Introducción 
Introducción 2 Capítulo 1. 
Capítulo 1. Introducción

1.1 Antecedentes

1.2 Fotosíntesis artificial

1.2.1. Ensamblaje molecular para fotosíntesis artificial.

1.3 Compuestos de coordinación de Ru para reacciones de interés ambiental

1.3.1 ¿Cómo actúan los complejos estudiados?

1.4 Otros diseños y usos de complejos con aplicaciones similares.

1.5 Referencias. 


\section{$1.1 \quad$ Antecedentes}

El consumo energético global ha ido creciendo rápidamente, haciendo indispensable la búsqueda de nuevas formas de energía que garanticen a las próximas generaciones una mejor calidad de vida. En la figura 1, se observa una proyección del incremento de la demanda mundial de energía entre los años 2010 y 2035 (World Energy Outlook, 2011).

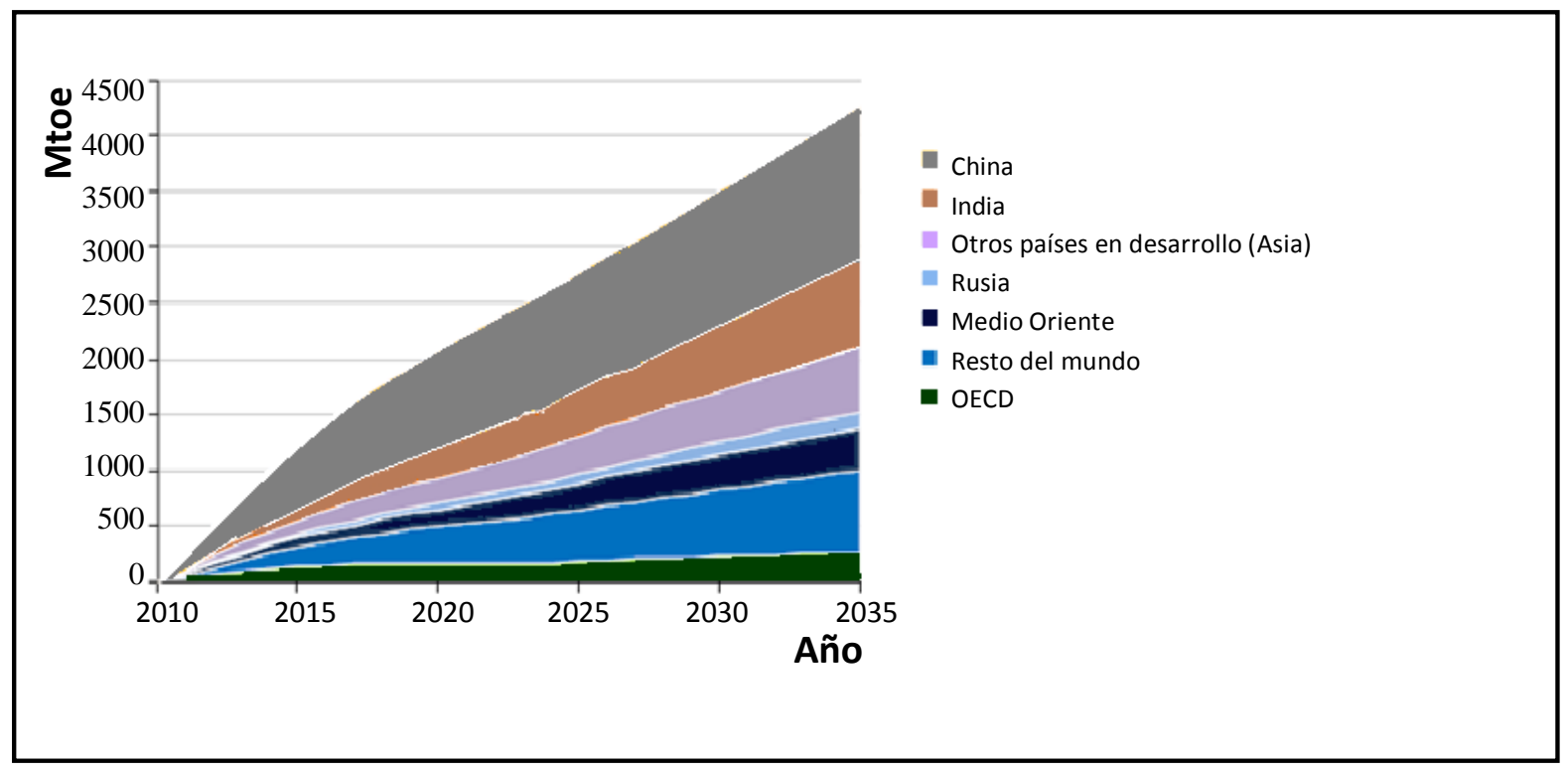

Figura 1. Proyección del incremento de la demanda mundial de energía entre los años 2010 y 2035. (World Energy Outlook, 2011). Mtoe: millones de toneladas equivalentes de petróleo. OECD: países miembros de la Organización Para Cooperación y el Desarrollo Económico

En la figura 2, se observa la distribución de las diferentes fuentes de abastecimiento energético a nivel mundial hasta el año 2009. La energía 
renovable utilizada fue aproximadamente $16 \%$ del consumo mundial de energía, contando con la biomasa tradicional y moderna, las energías hidroeléctrica, eólica, solar, geotérmica y los biocombustibles. La biomasa tradicional utilizada en países poco industrializados, representa aproximadamente el $10 \%$ de la cuota de energía renovable total. La energía hidroeléctrica corresponde a un $3.4 \%$ y crece lentamente. Otras energías renovables representan c.a. $2.8 \%$ con porcentaje creciendo rápidamente (REN21, 2011).

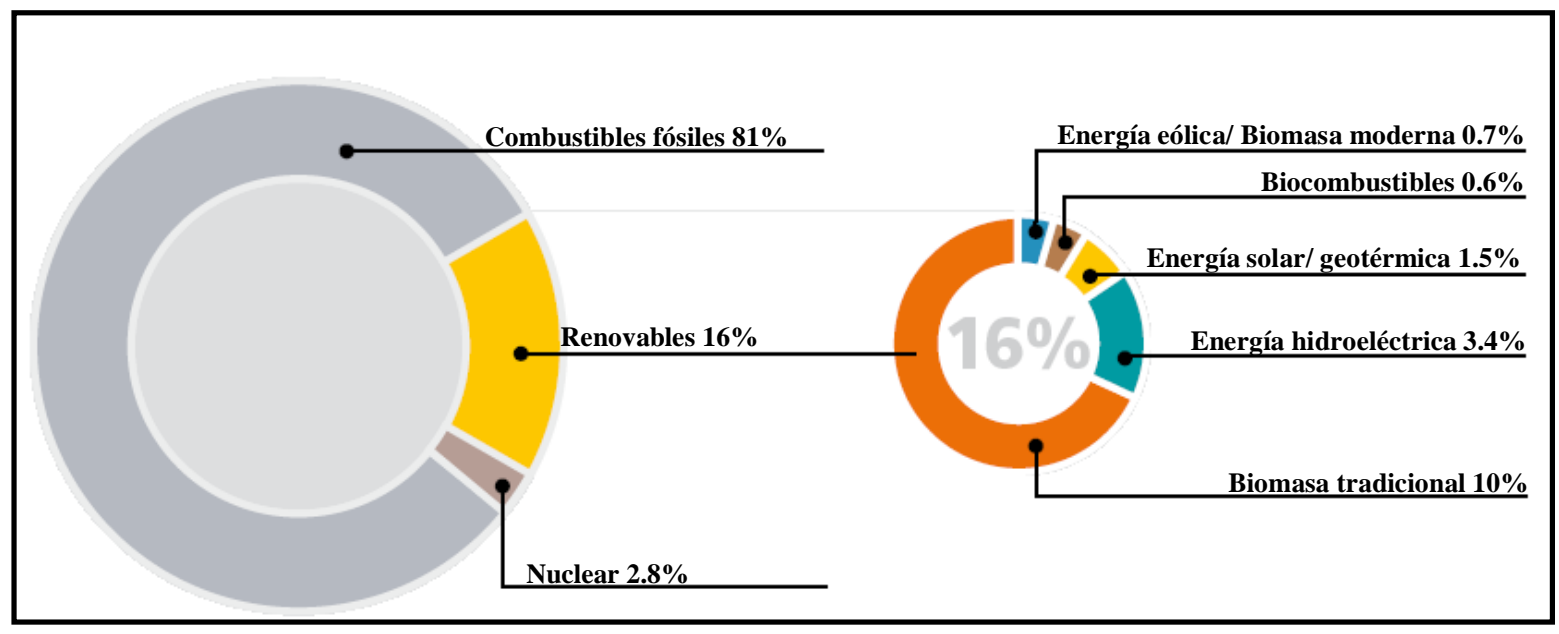

Figura 2. Fuentes que abastecen el consumo mundial de energía a 2009. (REN21, 2011)

El aumento de la calidad de vida en la población mundial en crecimiento, implica un incremento proporcional en el consumo energético. La demanda de energía y las limitaciones (prácticas y medioambientales) insalvables que poseen las fuentes tradicionales, conducen a que los seres humanos enfrenten problemas serios y urgentes como la variación global del clima, la contaminación ambiental y el agotamiento de los recursos naturales que se originan principalmente por el consumo de combustibles fósiles (Inagaki, A. et al., 2010). Bajo estas circunstancias los estudios sobre nuevos dispositivos para la conversión de energía se ha considerado una de las áreas de investigación más importantes, por lo tanto, un gran desafío tecnológico que enfrenta nuestro futuro global es el 
desarrollo de energías renovables (Dempsey, J. L. et al., 2005, Macor, L. et al., 2009).

En la búsqueda de este tipo de energías, el uso de energía solar es relevante debido a que es una fuente limpia, prácticamente inagotable y de libre disponibilidad. La luz solar incidente sobre la superficie de la Tierra $\left(\sim 1.2 \times 10^{5}\right.$ $\mathrm{TW} / \mathrm{h}$ ) es mucho mayor que la utilizada para todas las actividades humanas a nivel mundial durante un año. El sol es fuente de energía natural, impulsa la circulación de la energía eólica mundial, las corrientes oceánicas, el ciclo de evaporación del agua, la condensación que crea ríos y lagos, además de los ciclos biológicos de la fotosíntesis y la vida (Rajeshwar, K. et al., 2008).

La energía solar puede ser aprovechada de muchas maneras, especialmente para la conversión a energía eléctrica, química y térmica. El contenido energético de la radiación solar puede ser capturado como pares de electrones excitados - agujeros en un semiconductor, un colorante 0 un cromóforo, o en forma de calor en un medio de almacenamiento térmico. Los electrones excitados y los agujeros pueden ser aprovechados para la conversión inmediata a energía eléctrica, o transferidos a moléculas biológicas o químicas para la conversión a combustibles. La energía solar se fija en los vegetales a través del proceso fotosintético. Estas plantas están disponibles como biomasa para la conversión a combustibles primarios o para la formación a combustibles secundarios tales como etanol o hidrógeno. (Rajeshwar, K. et al., 2008).

La fotosíntesis natural muestra que es posible construir sistemas moleculares o supramoleculares que pueden capturar y almacenar energía en moléculas tales como ATP (adenosín trifosfato), NADPH (Nicotinamida-AdeninaDinucleótido-Fosfato) y glucosa. Sin intentar replicar la complejidad de los sistemas naturales, es posible diseñar sistemas fotosintéticos artificiales que 
mimeticen los procesos básicos de la naturaleza con moléculas mucho más simples que ATP, NADPH o glucosa; por ejemplo, con compuestos de coordinación diseñados específicamente para la "descomposición del agua" en los productos deseados $\mathrm{H}_{2}$ y $\mathrm{O}_{2}$. El hidrógeno renovable es ampliamente considerado como un posible vector de energía para la era de combustibles post-fósiles aunque aún restan numerosos problemas prácticos a resolver para que una economía de hidrógeno sea posible, particularmente con relación a la generación en forma eficiente y barata a partir de $\mathrm{H}_{2} \mathrm{O}$ mediante radiación solar.

\subsection{Fotosíntesis Artificial}

El objetivo de la fotosíntesis artificial es simular el funcionamiento de plantas verdes y otros organismos fotosintéticos que utilizan luz solar para producir sustancias químicas de alta energía. Esta es una meta difícil, porque el éxito requiere de la integración de funciones químicas múltiples en una arquitectura química estable. Como resultado, la fotosíntesis artificial se ha desarrollado más lentamente que otros enfoques de la conversión de energía solar, es decir, celdas fotovoltaicas, celdas solares, y dispositivos orgánicos de película delgada (Alstrum - Acevedo, J.H., Meyer, T. et al., 2005).

La fotosíntesis natural de plantas verdes superiores posee un esquema de reacción complejo que utiliza energía solar para obtener $\mathrm{O}_{2}$ a partir de $\mathrm{H}_{2} \mathrm{O}$ y equivalentes de reducción que aparecen como NADPH. En el llamado fotosistema I (Alstrum - Acevedo, J.H., Meyer, T. et al., 2005, Rajeshwar, K. et al., 2008) los equivalentes de reducción NADPH se utilizan para reducir $\mathrm{CO}_{2}$ a carbohidratos (reacción 1). El interés en fotosíntesis artificial, es el aprovechamiento de la energía solar para impulsar reacciones de moléculas pequeñas de alta energía como la descomposición del agua (reacción 2) o la reducción de $\mathrm{CO}_{2}$ para 
producir carbohidratos y $\mathrm{O}_{2}$ (reacción 3) (Alstrum - Acevedo, J.H., Meyer, T. et al., 2005, Rajeshwar, K. et al., 2008).

$$
\begin{aligned}
& 6 \mathrm{H}_{2} \mathrm{O}+6 \mathrm{CO}_{2}+48 h v \rightarrow \mathrm{C}_{6} \mathrm{H}_{12} \mathrm{O}_{6}+6 \mathrm{O}_{2} \\
& \mathrm{H}_{2} \mathrm{O}_{(l)}+2 h v \stackrel{\text { Fotocataliador }}{\rightarrow} \mathrm{H}_{2(g)}+1 / 2 \mathrm{O}_{2(g)} \\
& 2 \mathrm{H}_{2} \mathrm{O}+2 \mathrm{CO}_{2}+4 h v \rightarrow 2 \mathrm{HCOOH}+\mathrm{O}_{2}
\end{aligned}
$$

Los productos químicos de alta energía que se forman en estas reacciones pueden ser recombinados para extraer su energía química almacenada.

La reacción 2 que corresponde a la descomposición fotocatalítica del agua, es tal vez, una de las reacciones más importantes de la fotosíntesis artificial ya que utiliza energía solar (fuente limpia y renovable de energía) para generar hidrógeno y oxígeno, los que luego se emplean como combustibles en celdas para generar electricidad (Esquema 1).

$$
\begin{aligned}
& \text { Fotoquímica: } \quad 2 \mathrm{H}_{2} \mathrm{O}+4 h v \stackrel{\text { Fotocataliador }}{\rightarrow} 2 \mathrm{H}_{2}+\mathrm{O}_{2} \\
& \text { Celda de combustible : } \quad 2 \mathrm{H}_{2} \rightarrow 4 \mathrm{H}^{+}+4 e^{-} \text {(ánodo) } \\
& \frac{\mathrm{O}_{2}+4 \mathrm{H}^{+}+4 e^{-} \rightarrow 2 \mathrm{H}_{2} \mathrm{O}(\text { cátodo })}{2 \mathrm{H}_{2}+\mathrm{O}_{2} \rightarrow 2 \mathrm{H}_{2} \mathrm{O}: \Delta G=-4.92 \mathrm{eV}(-113 \mathrm{kcal} / \mathrm{mol})}
\end{aligned}
$$

Esquema 1. Descomposición del agua acoplada a una celda de combustible hidrógeno/oxígeno.

Las reacciones de almacenamiento de energía (reacciones 1-3) son del tipo de oxido-reducción (redox) y pueden dividirse en hemirreacciones. Cada 
hemirreacción implica un intercambio múltiple de electrones. La energía correspondiente a la intervención de $1 \mathrm{e}^{-}$en intermediarios tales como $\mathrm{CO}_{2}{ }^{\circ-} \mathrm{o}$ $\cdot \mathrm{OH}$, muestra que tanto las reacciones fotoquímicas para la formación de combustible como las recombinaciones posteriores, son procesos lentos en condiciones ambientales. Ambas hemirreacciones deben ser catalizadas para que puedan producirse a mayor velocidad o a una temperatura cercana a la ambiente (Alstrum - Acevedo, J.H., Meyer, T. et al., 2005).

Las hemirreacciones descriptas, proporcionan la base para proponer el modelo de fotosíntesis artificial (Esswein, A. J. et al., 2007). Al igual que en la fotosíntesis natural, cada hemirreacción se puede conducir por separado y en una etapa posterior se pueden combinar en un único dispositivo. La complejidad para generar este acoplamiento es inevitable debido a los requerimientos multifuncionales (absorción de la luz, transferencia de energía, transferencia de electrones, catalizadores redox) (Amouyal, E., 1995), por lo tanto se requiere organizar e integrar los grupos funcionales y proporcionar una estructural global ordenada (Alstrum - Acevedo, J.H., Meyer, T. et al., 2005).

La búsqueda de esta estructura, conduce al concepto de "ensamble molecular integrado" como se describe en Meyer, T. J., 1989 y más recientemente en Sykora, M. et al., 2000, Alstrum - Acevedo, J.H., Meyer, T. et al., 2005, Huynh, M H. V. et al., 2005, MacDonnell, F. M., 2008, Inagaki, A. et al., 2010. Las investigaciones realizadas en esta área poseen dos enfoques; uno trata de estudiar las porfirinas y metaloporfirinas como cromóforos y su incorporación en ensambles moleculares (Gust, D. et al., 1999, 2001, Fungo, F. et al., 2001) y el segundo se basa en los estados excitados de transferencia de carga metalligando (MLCT) de complejos metálicos polipiridilos (Alstrum - Acevedo, J.H., Meyer, T. et al., 2005, Huynh, M H. V. et al., 2005, Sykora, M. et al., 2000, Campagna, S. et al., 2007, MacDonnell, F. M., 2008, Inagaki, A. et al., 2010, 
Matsubara, Y. et al. 2010); ambos métodos utilizan los mismos principios físicos y técnicas similares para el ensamblaje molecular.

\subsubsection{Ensamblaje molecular para fotosíntesis artificial.}

Hay muchas formas para organizar los distintos componentes dentro del ensamblaje para fotosíntesis artificial. Una de ellas es utilizar soportes de polímero (Sykora, M. et al., 2000) incluyendo proteínas (Cristian, L. et al., 2003), para sostener y organizar los diversos componentes. Otra es utilizar una celda de síntesis fotoelectroquímica (PES) (Alstrum - Acevedo, J.H., Meyer, T. et al., 2005) (Figura 4) para realizar las hemirreacciones por separado. En una celda PES, el potencial es proporcionado por la luz donde la absorción y la posterior transferencia de electrones en el estado excitado se producen en un dispositivo molecular fotoactivo que vincula a los dos electrodos. El material fotoactivo puede estar hecho de un semiconductor (Fujishima, A. et al., 1972, Fujihara, K. et al., 1998, Khaselev, O. et al., 1998), un complejo de metales de transición o una combinación de ambos (Grätzel, M., 2001). Otro método es organizar los diferentes grupos funcionales en un ensamble supramolecular unidos por fuerzas de atracción intra o intermoleculares.

La figura 3 muestra elementos y procesos requeridos en un ensamble molecular para fotosíntesis artificial y la secuencia de eventos que ocurren después de la absorción de luz.

Los elementos utilizados son:

- Una antena de absorción de luz.

- Un sitio sensibilizado por la antena llamado cromóforo (C)

- Un donor (D) y un aceptor (A), para la transferencia de electrones. 
- Catalizadores moleculares para la reducción (Cat ${ }_{\text {red }}$ y oxidación (Cat ox $_{\text {) }}$ de la reacción de interés. (La reacción mostrada en la figura 3 ilustra la descomposición fotoquímica del agua en $\mathrm{H}_{2}$ y $\mathrm{O}_{2}$.). (Konduri, $R$. et al., 2002, Huynh, M H. V. et al., 2005)

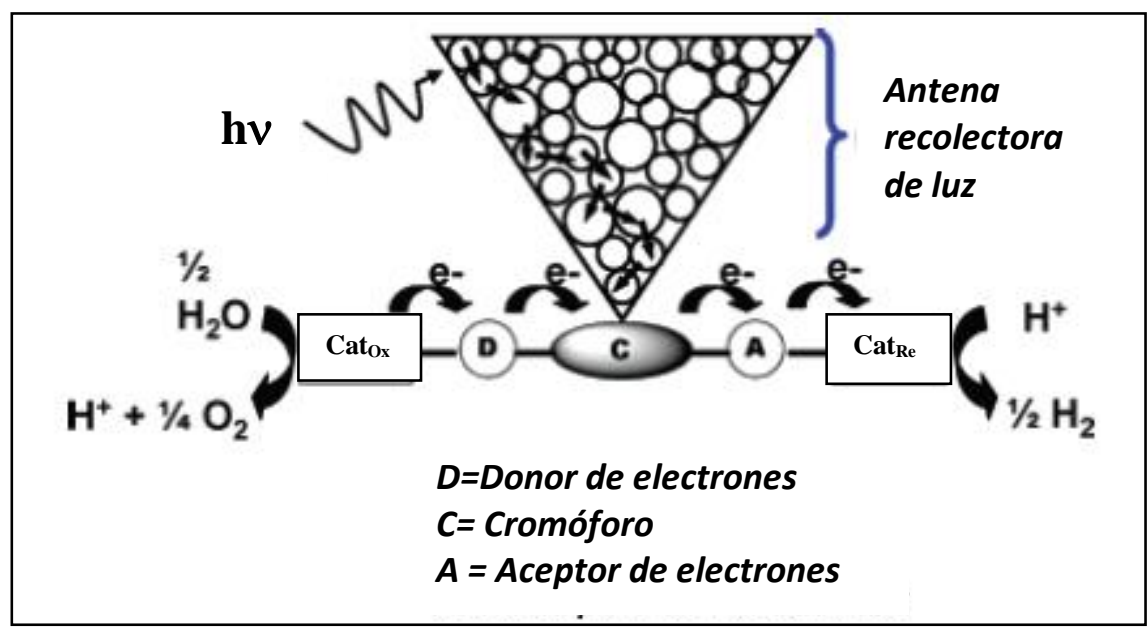

Figura 3. Diagrama básico de un ensamble molecular para fotosíntesis artificial.

Aunque el diseño y fabricación de este tipo de sistemas es una tarea bastante compleja, hay que tener en cuenta que mediante fotosíntesis natural, las plantas superiores realizan procesos como oxidación del agua y reducción de $\mathrm{CO}_{2}$, lo cual es motivo de inspiración y motivación para su estudio (Huynh, $M \mathrm{H}$. V. et al., 2005).

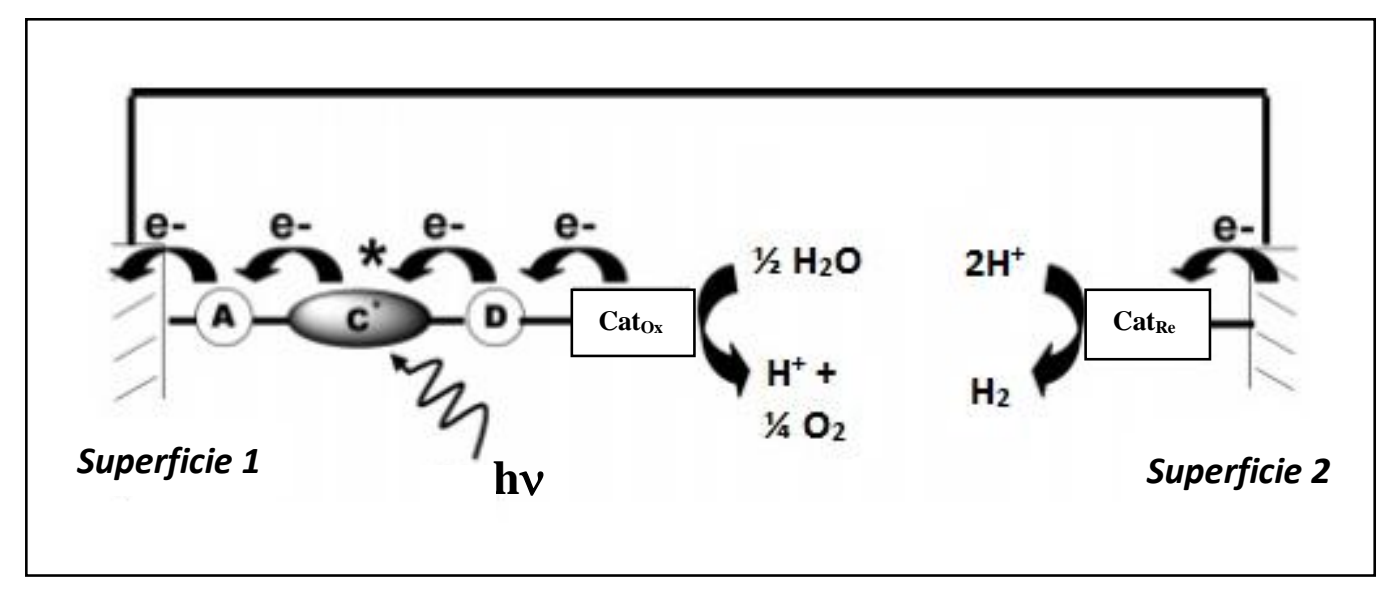

Figura 4. Diagrama esquemático para una celda PES. 
Como se mencionó anteriormente, no se intenta replicar la complejidad de los sistemas naturales, una propuesta más simple podría ser obtener $\mathrm{H}_{2}$ y $\mathrm{O}_{2}$, idealmente a partir de $\mathrm{H}_{2} \mathrm{O}$ como sustrato, i.e. en la descomposición del agua (reacción 2). A pesar de que el $\mathrm{H}_{2}$ es considerado un combustible ideal para la era post-fósil, por no poseer carbono y por su alto contenido de energía por unidad de masa (hidrógeno + aire $\rightarrow$ celda de combustible), existen numerosos inconvenientes para que una economía de hidrógeno sea posible, debido particularmente al requerimiento de generación en forma eficiente y barata de hidrógeno a partir de $\mathrm{H}_{2} \mathrm{O}$ mediante radiación solar (utilizando fotosíntesis artificial).

Desde el punto de vista de la fotocatálisis, el interés se ha enfocado en el uso de Pt unido a partículas semiconductoras. Sin embargo, éste material posee varios inconvenientes (Reisner, E. et al., 2009) a saber:

- Pobre selectividad para la generación de hidrógeno, debido a la competencia que existe a potenciales negativos con la reacción de reducción de $\mathrm{O}_{2}$.

- Envenenamiento difícil de tratar, por trazas de inhibidores (e.g. CO adsorbido).

- Alto costo y fuentes limitadas para aplicaciones a gran escala (Linsebigler, A. L. et al., 1995).

Los fotocatalizadores que pueden promover la descomposición del agua (reacción 2) están aun en desarrollo, sin embargo, se puede minimizar el problema si se divide esta reacción en dos hemirreacciones (de manera similar al mostrado en el esquema 1), donde HER corresponde a la reacción de desprendimiento de hidrógeno y OER al desprendimiento de oxígeno (esquema 2). 


$$
\begin{aligned}
& \text { Descomposición del agua: } 2 \mathrm{H}_{2} \mathrm{O} \stackrel{\text { Fotocatalzador }}{\rightarrow} 2 \mathrm{H}_{2}+\mathrm{O}_{2} \quad(+113 \mathrm{kcal} / \mathrm{mol}) \\
& \text { HER: } \quad 2 \mathrm{H}^{+}+2 e^{-} \rightarrow \mathrm{H}_{2} \quad(+19,08 \mathrm{kcal} / \mathrm{mol}) \\
& \text { OER: } \quad 2 \mathrm{H}_{2} \mathrm{O} \rightarrow \mathrm{O}_{2}+4 \mathrm{H}^{+}+4 e^{-}(+75,21 \mathrm{kcal} / \mathrm{mol})
\end{aligned}
$$

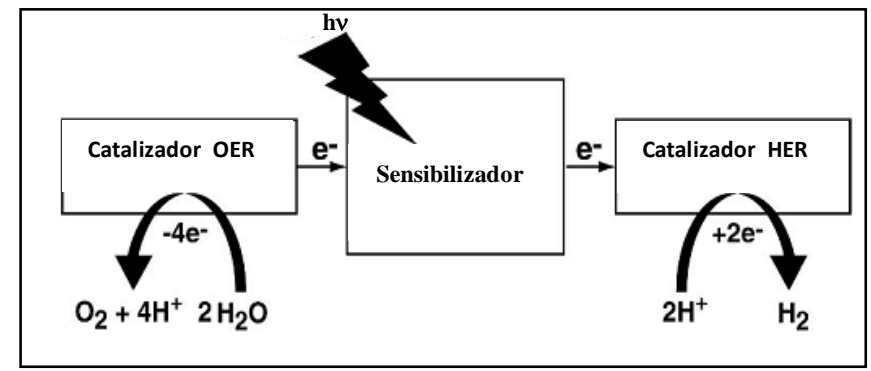

Esquema 2. Esquema de un sistema fotosintético artificial relacionado con la descomposición del agua.

Un fotocatalizador básico para este proceso tendría las funciones descriptas en la figura 3 , donde un fotosensibilizador captura fotones y da lugar a la separación de cargas, $\mathrm{e}^{-} / \mathrm{h}^{+}$. Los electrones son tomados por un aceptor $\mathbf{A}$ y los huecos son llenados por un donor $\mathbf{D}$. Por lo tanto, Las especies $\mathbf{A}^{-}$y $\mathbf{D}^{+}$se acoplan a los catalizadores para promover las reacciones (HER) y (OER) respectivamente (esquema 2 ).

Nuestro interés está dirigido a una de las hemirreacciones, la HER. La naturaleza multielectrónica de la reacciones HER y OER presentes en el esquema 2, no coinciden con las propiedades de la mayoría de los fotosensibilizadores estudiados (por ejemplo porfirinas, metaloporfirinas y ftalocianinas (Gust, D. et al., 1999, 2001), complejos de metales de transición de Ru, Os, Re, Rh, Pt, Cu, etc.), los cuales generalmente en la mayoría de las excitaciones moleculares están limitados a la creación de un único par $\mathrm{e}^{-} / \mathrm{h}^{+}$(Cukier, R. I. et al., 1998, Heyduk, A. F. et al., 1999, 2001). Por lo tanto es esencial diseñar aceptores, para el caso 
de la HER, que sean capaces de almacenar al menos 2 electrones si se pretende generar hidrógeno.

\subsection{Compuestos de coordinación de $\mathrm{Ru}$ para reacciones de interés ambiental.}

En trabajos previos se han investigado complejos de Rutenio (II) con ligandos polipiridilos (Figura 5), que poseen cromóforos muy estudiados debido a su estabilidad química y a sus favorables propiedades electroquímicas y fotofísicas (Kim, M-J. et al., 2002, Chao, H. et al., 2003, Gut, D. et al., 2003, Konduri, R. et al., 2004, Tacconi, N. R., Lezna, R.O. et al., 2005). Este tipo de complejos juega un papel importante en el desarrollo de sistemas artificiales de múltiples componentes (supramoleculares) para la conversión de energía solar a energía eléctrica. (Kim, M-J. et al., 2002, Gut, D. et al., 2003).

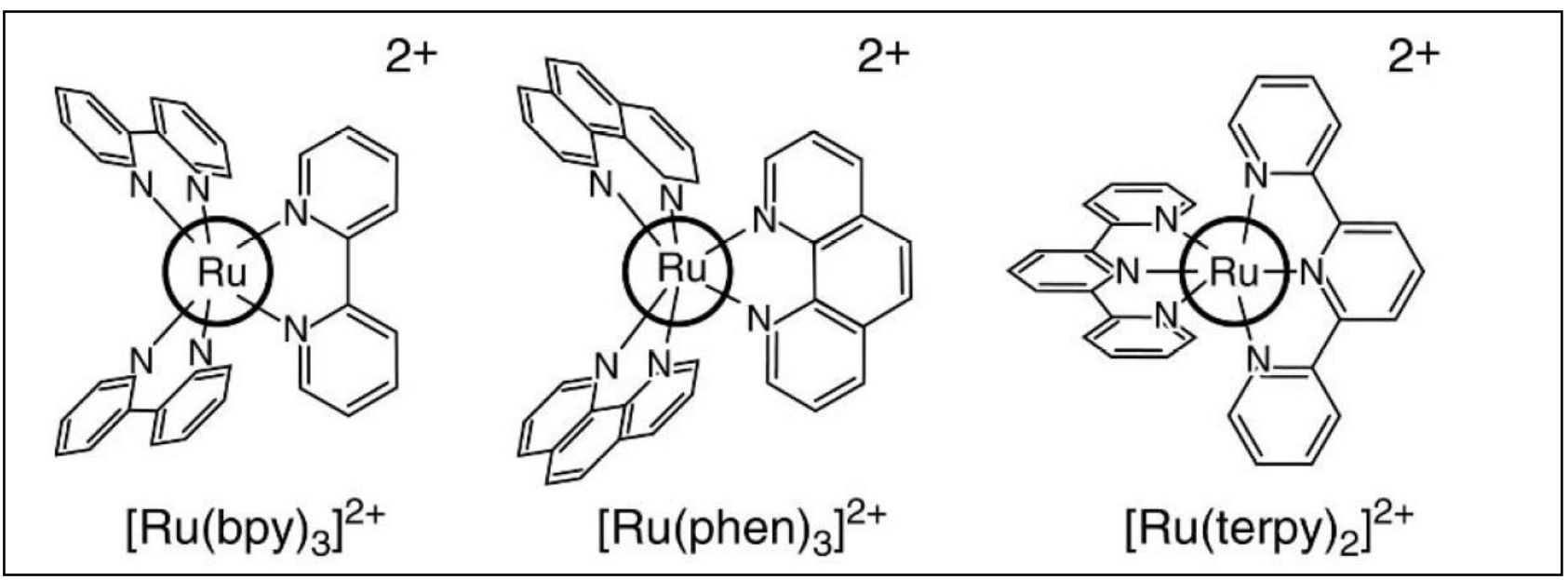

Figura 5. Estructura química de tres complejos de $R u(I I)$ polipiridil comúnmente usados.

(MacDonnell, F. M., 2008)

En los últimos años, se ha desarrollado una variedad de ligandos puente para ensamblarlos a las estructuras de polipiridil Ru (II) (De Cola, L. et al., 1998, 
Barigelletti, F. et al., 2000). Sin embargo, el interés se ha centrado en los sistemas que contienen ligandos puentes simétricos (Chao, H. et al., 2003).

En la mayoría de los casos, las actividades redox y las propiedades del estado excitado de los complejos estudiados, son dependientes del tamaño, forma y naturaleza electrónica del ligando puente. Así, el diseño sintético de éste ligando es uno de los pasos claves en la realización de dispositivos moleculares basados en complejos polinucleares de Ru (II) (Chao, H. et al., 2003).

En nuestro grupo de investigación se ha realizado últimamente el estudio de complejos mono y dinucleares de $\mathrm{Ru}$, con características estructurales similares a las mostradas en la figura 6. Las propiedades de estos complejos permiten compararlos, en general, con los procesos fotosintéticos artificiales, debido a que poseen distintos grupos funcionales que actúan como antenas (Ruphen) encargadas de absorber fotones y como almacenadores electrónicos (ligando central) como describe la figura 6.
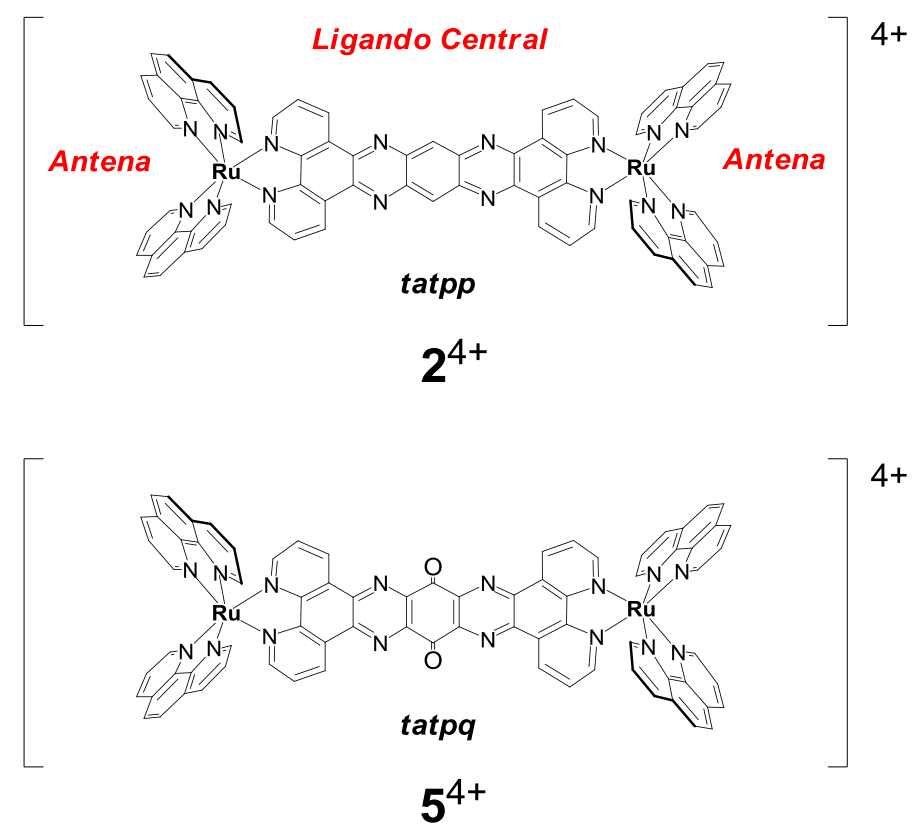

Figura 6. Estructura de dos complejos modelo $2^{4+}$ y $5^{4+}$. 
Los complejos de $\mathrm{Ru}$ (II), mostrados en la figura 6 corresponden a $2^{4+}$ y $5^{4+}$ que bajo reducciones fotoinducidas (irradiación con luz visible) en acetonitrilo y en presencia de un reductor de sacrificio son capaces de almacenar reversiblemente 2 y 4 electrones respectivamente (Konduri, $R$. et al., 2002, 2004). $2^{4+}$ y $5^{4+}$ están compuestos por 2 grupos trisfenantrolina rutenio (II) unidos por el ligando puente central tetraazapentaceno (tatpp) o tetraazapentaceno quinona (tatpq) respectivamente.

A partir de las investigaciones realizadas con estos complejos, se demostró que el ligando puente central (tatpp y tatpq) juega el papel de subunidad aceptora de electrones de los procesos de separación de carga fotoinducida y también asume el rol de unidad de almacenamiento multielectrónico (Konduri, R. et al., 2004).

\subsection{1 ¿Cómo actúan los complejos estudiados?}

Los complejos mencionados poseen antenas y un ligando puente central. La antena actúa como cromóforo, capturando luz visible ( $\lambda_{\operatorname{máx}} 480 \mathrm{~nm}$, MLCT) y los ligandos puente (tatpp y tatpq) poseen aceptores internos o sitios de almacenamiento multielectrónico (figura 6); estos ligandos puente, exhiben similitudes estructurales con componentes de sistemas naturales que sustentan procesos fotosintéticos fundamentales.

El estudio de la capacidad de almacenamiento electrónico para cada complejo se realiza, en los experimentos fotoquímicos, en presencia de agentes reductores de sacrificio e.g. trietilamina (TEA). McDonnell y colaboradores han demostrado que los complejos $2^{4+}$ y $5^{4+}$ pasan por 2 y 4 reducciones para dar $\mathrm{H}_{2} 2^{4+}$ y $\mathrm{H}_{4} 5^{4+}$ respectivamente (figura 7). Las fotoreducciones ocurren en pasos 
que involucran un solo electrón bajo condiciones normales (MacDonnell, F. M., 2008).
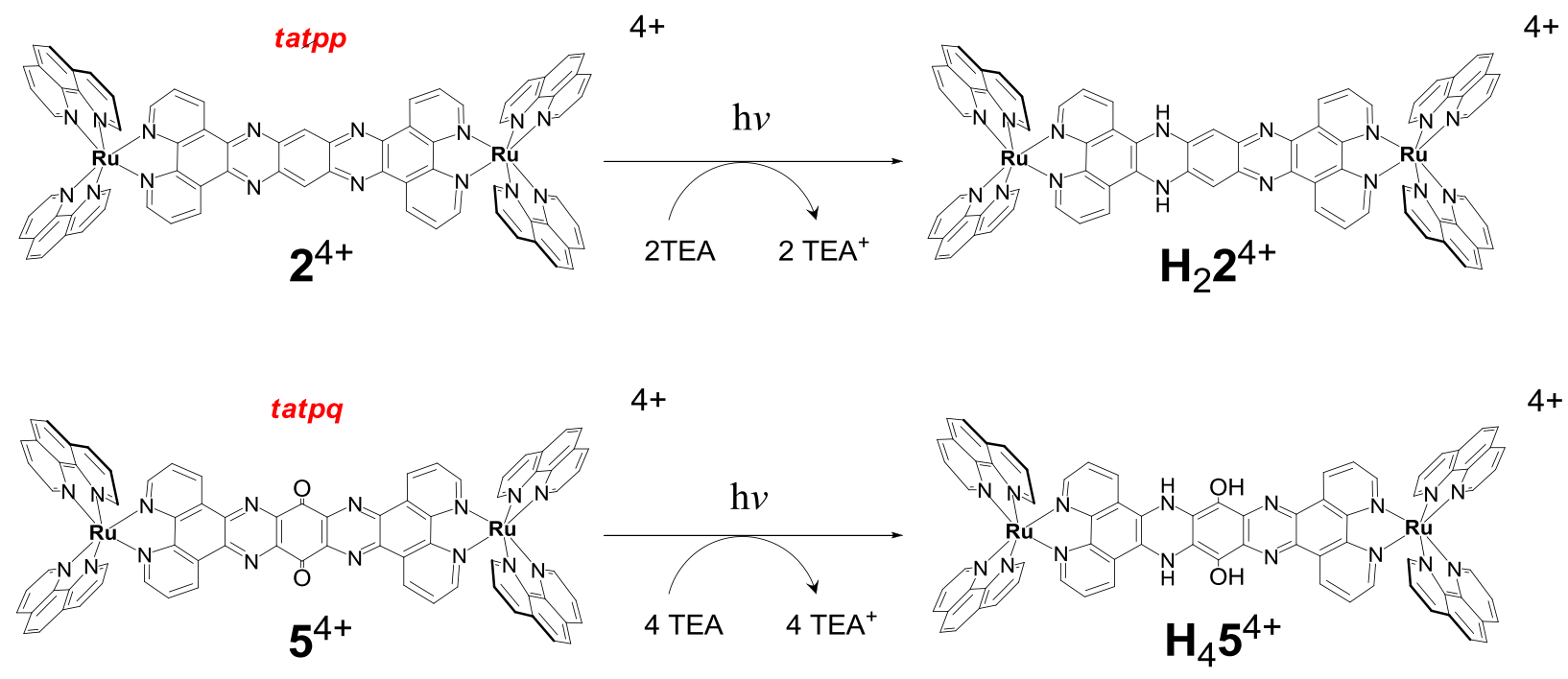

Figura 7. Fotoreducciones para $2^{4+}$ y $5^{4+}$ (MacDonnell, F. M., 2008)

Bajo luz visible y en presencia de un reductor de sacrificio, estos complejos se reducen, incorporando hidrógeno en sus estructuras (figura 7). En la figura 8 se muestra el proceso, ejemplificado para el complejo $2^{4+}$, en donde luego de su reducción y en presencia de un co-catalizador metálico como $\mathrm{Pt}$, se genera $\mathrm{H}_{2}$.

Experimentalmente, lo que se realiza en el laboratorio es la reducción electroquímica (por diferentes métodos), del complejo a estudiar, en medio orgánico (acetonitrilo) utilizando hexafluorofosfato de tetrabutilamonio como electrolito soporte. 


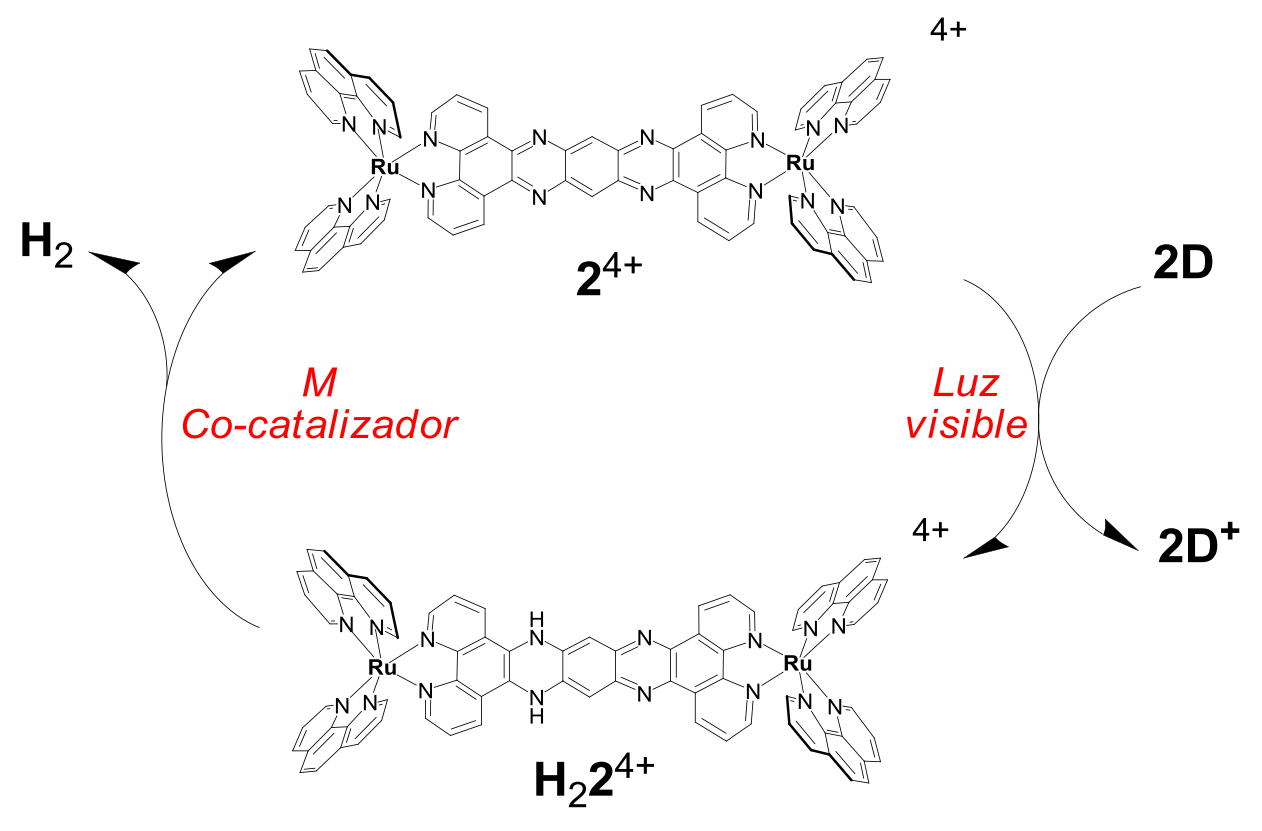

Figura 8. Esquematización de la catálisis del desprendimiento de $\mathrm{H}_{2}$

En nuestro grupo de investigación, se estudian las propiedades de complejos de Ru que contienen ligandos puente tatpp, tatpq y de otros complejos relacionados. En el contexto de este trabajo se analizaron los complejos y el ligando listados en la tabla 1.

Tabla 1. Compuestos de coordinación de Ru estudiados

\begin{tabular}{c|c|c|c}
\hline Antena & $\begin{array}{c}\text { Ligando } \\
\text { puente }\end{array}$ & Denominación & Estructura \\
\hline $\begin{array}{c}2[\mathrm{Ru}- \\
\left.\text { phen }_{2}\right]\end{array}$ & tatppo & &
\end{tabular}




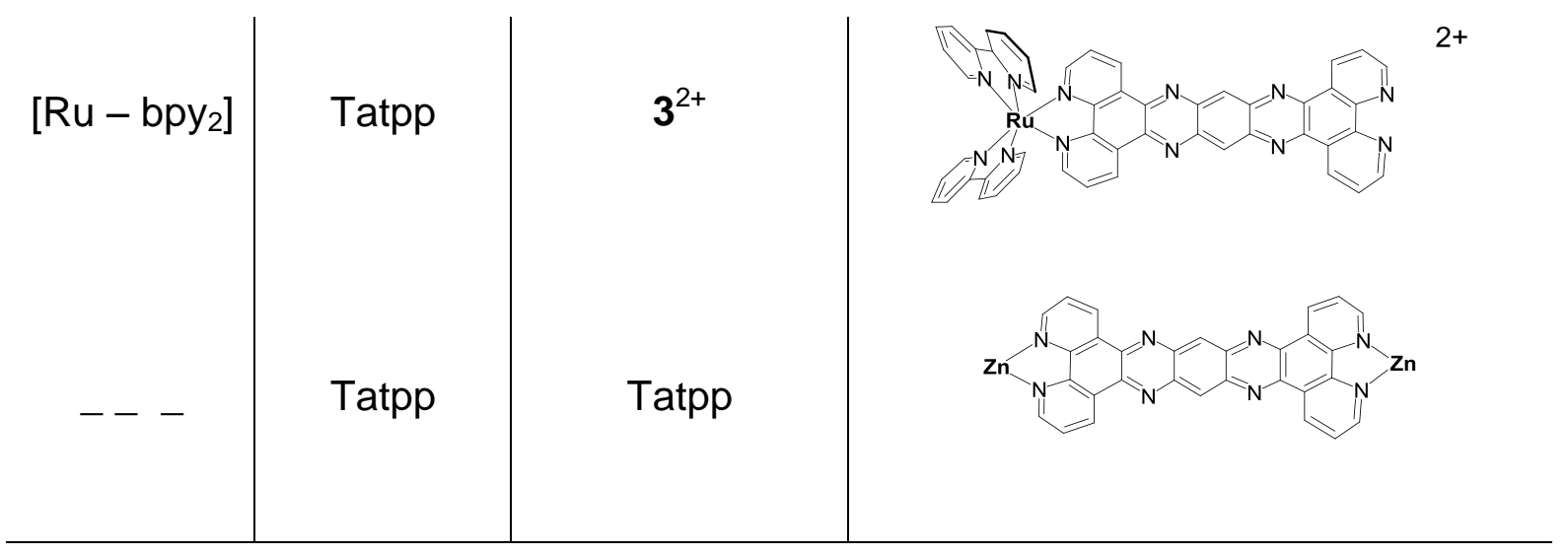

\subsection{Otros diseños y usos de complejos con aplicaciones similares.}

Como se citó anteriormente, estos complejos pueden actuar como fotocatalizadores para la generación de hidrógeno; además de esto, se ha demostrado que complejos con características similares, catalizan la electroreducción de $\mathrm{CO}_{2}$. La función que puede llegar a cumplir cada complejo depende principalmente de su diseño.

El diseño de los sistemas a estudiar se realiza con la intención de adaptarlo a un fin perdeterminado con la mayor eficiencia posible. Por ejemplo, actualmente se están rediseñando estructuras respecto a las mostradas en la figura 6 , adicionando grupos donores al ligando central como por ejemplo $-\mathrm{C} \equiv \mathrm{N}^{-}$(figura 9), con el fin de disminuir el potencial (hacerlo menos negativo) para reducir $\mathrm{CO}_{2}$ (Cole, E.B., Bocarsly, A.B. et. al., 2010 a y b). 


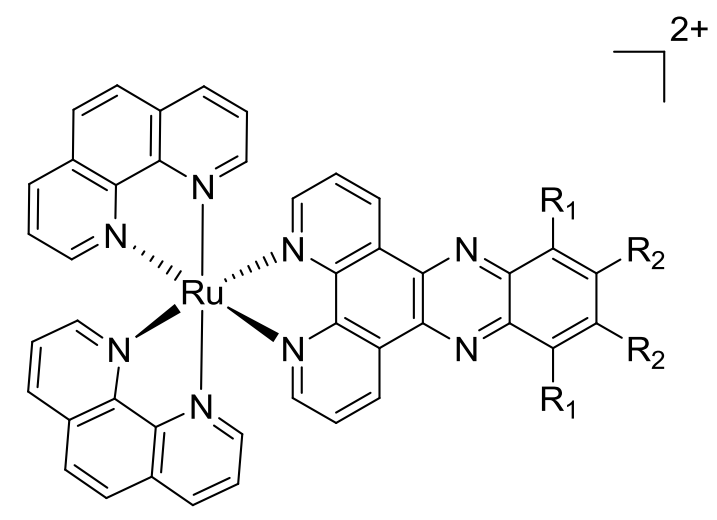

Figura 9. Diseño de nuevas estructuras donde $R_{1}$ y $R_{2}$ corresponden a grupos $\mathrm{CN}^{-}$

En adición al estudio de estos complejos, también se realizaron medidas de polímeros inorgánicos con propiedades diferentes a los mostrados anteriormente. En el capítulo 5 se analizan este tipo de complejos, en su función como fotocatalizadores, principalmente en dispositivos ópticos y en el proceso de reducción de $\mathrm{CO}_{2}$. 


\section{Referencias}

Alstrum - Acevedo, J.H.; Brennaman, M. K.; Meyer, T. J. Inorg. Chem. 2005, 44, $6802-6827$.

Amouyal, E. Solar Energy Materials and Solar Cells, 1995, 38, 249 - 276.

Barigelletti, F.; Flamingni, L. Chem Soc. Rev. 2000, 29, 1 - 12.

Campagna, S.; Puntoriero, F.; Nastasi, F.; Bergamini, G.; Balzani, V. Top. Curr. Chem. 2007, 280, 117 - 214.

Chao, H.; Qiu, Z-R.; Cai, L-R.; Zhang, H.; Li, X-Y.; Wong, K-S. y Ji, L-N. Inorg. Chem. 2003, 42, $8823-8830$.

Cole, E.B.; Lakkaraju, P.S.; Rampulla, D. M.; Morris, A. J.; Abelev, E. y Bocarsly A.B. Journal American Chemistry. 2010 a,132, 11539 - 11551.

Cole, E.B. y Bocarsly A.B. Photochemical, Electrochemical and Photoelectrochemical Reduction of Carbon Dioxide, en "Carbon Dioxide as Chemical Feedstock, 2010 b, editado por Michele Aresta, WILEY-VCH, Cap. 11.

Cristian, L.; Piotrowiak, P. y Farid, R. S. J. Am. Chem. Soc. 2003, 125, 11814 $-11815$.

Cukier, R. I.; Nocera, D. G. Ann. Rev. Phys. Chem. 1998, 49, 337 - 369. 
De Cola, L.; Belser, P. Coord. Chem. Rev. 1998, 177, 301- 346.

Dempsey, J. L.; Esswein, A. J.; Manke, D. R.; Rosenthal, J.; Soper, J. D. y Nocera, D. G. Inorg. Chem. 2005, 44, 6879 - 6892.

Esswein, A. J. y Nocera, D. G. Chemical Reviews. 2007, 107, 4022 - 4047.

Fujishima, A.; Honda, K. Nature 1972, 238, (5358), 37 - 38.

Fujihara, K.; Ohno, T. y Matsumura, M. J. Chem. Soc., Faraday Trans. 1998, 94 (24), 3705 - 3709.

Fungo, F.; Otero, L.; Durantini, E. N.; Silber, J. J.; Sereno, L.; Mariño-Ochoa, E.; Moore, T. A.; Moore, A. L. y Gust, D. J. Phys. Chem. B. 2001, 105, 4783 4790.

Grätzel, M. Nature 2001, 414, 338 - 344.

Gust, D.; Moore, T. A. Intramolecular photoinduced electron-transfer reactions of porphyrins. In The Porphyrin Handbook; Academic Press: New York, 1999, $153-190$.

Gust, D.; Moore, T. A.; Moore, A. L. Acc. Chem. Res. 2001, 34, 40 - 48.

Gut, D.; Goldberg, I. y Kol, M. Inorg. Chem. 2003,42, 3483 - 3491.

Heyduk, A. F.; Macintosh, A. M.; Nocera, D. G. J. Am. Chem. Soc. 1999, 121, $5023-5032$.

Heyduk, A. F.; Nocera, D. G. Science, 2001, 293, 1639 - 1641.

Huynh, MH. V.; Dattelbaum, D. M.; Meyer. J. Coordination Chemistry Reviews. 2005, 249, 457 - 483. 
Inagaki, A. y Akita, M. Coordination Chemistry Reviews, 2010, 254, 1220 1239.

Khaselev, O. y Turner J. A. Science. 1998, 280, 425 - 427.

Kim, M-J.; Konduri, R.; Ye, H.; MacDonnell, F.M.; Puntoriero, F.; Serroni,S.; Campagna, S.; Holder, T.; Kinsel, G. y Rajeshwar, K. Inorg. Chem. 2002, 41, $2471-2476$.

Konduri, R.; Ye, H.; MacDonnell, F. M.; Serroni,S.; Campagna,S. y Rajeshwar, K. Angew. Chem. 2002, 41,(17) 3185 - 3187.

Konduri, R.; de Tacconi, N. R.; Rajeshwar,K.; y MacDonnell, F. M. J. Am. Chem. Soc. 2004, 126, 11621-11629.

Linsebigler, A. L.; Lu,G. y Yates, J.T. Jr. Chemical Reviews, 1995, 95, (3) $735-758$.

Macor, L.; Natera, J.; Heredia, D.; Fernandez, L.; Santos, M.; Gervaldo, M.; Fungo, F. y Otero, L. Avances en Energías Renovables y Medio Ambiente, 2009, 13, $04.63-04.60$.

MacDonnell, F. M., Solar Hydrogen Generation - Toward a Renewable Energy Future, 2008, editorial: Springer: New York, capitulo 6.

Matsubara, Y.; Koga, K.; Kobayashi,A.; Konno,H.; Sakamoto, K.; Morimoto, T. y Ishitani, O. J. Am. Chem. Soc. 2010, 132, 10547 - 10552.

Meyer, T. J. Acc. Chem. Res, 1989, 22, 163. 
Sykora, M.; Maxwell, K. A.; DeSimone, J. M.; y Meyer, T. J. Proc. Nat. Acad. Sci. 2000, 97,(14) 7687 - 7691.

Rajeshwar, K.; McConnell, R.; Harrison, K. y Licht, S. Solar Hydrogen Generation -Toward a Renewable Energy Future, 2008, Springer: New York, capítulo 1.

Reisner, E.; Fontecilla - Camps, J.C. y Armstrong, F.A. Chem. Commun., 2009, $550-552$.

Ren21, Renewables 2011 global status report. 2011, Paris: REN21 secretariat. Ver también referencias incluidas.

http://www.ren21.net/Portals/97/documents/GSR/REN21 GSR2011.pdf

Tacconi, N. R.; Lezna, R. O.; Konduri, R.; Ongeri, F.; Rajeshwar, K. y MacDonnell, F. M. Chem. Eur. J. 2005, 11, 4327 - 4339.

World Energy Outlook, International Energy Agency, noviembre de 2011. http://www.worldenergyoutlook.org/media/weowebsite/2011/WEO2011 Press Launch London.pdf 
2. Materiales y métodos 
Materiales y Métodos 2 Capítulo 2. 
Capítulo 2. Materiales y Métodos.

2.1 Parte experimental

$2.2 \quad$ Medidas electroquímicas

2.2.1 La doble capa eléctrica

2.2.2 Cinética electroquímica

2.2.3 Determinación de la cinética de electrodos

2.2.4 Pulsos de potencial

2.2.5 Voltamperometría

2.2.5.1 Voltamperometría de corriente continua - DCV

2.2.5.2 Voltamperometría de corriente alterna - ACV

$2.3 \quad$ Medidas Ópticas In-Situ

2.3.1 Espectroscopía UV-vis - (OTTLEs)

2.3.2 Reflectancia Total Atenuada (ATR)

2.3.3 Espectroscopía de reflectancia modulada especular

2.3.3.1 Reflectancia externa especular

2.3.4 Espectroscopía de reflectancia para especies adsorbidas sobre la superficie del electrodo

2.3.5 Espectroscopía de reflectancia de especies en solución

2.3.6 Medidas diferenciales

2.3.7 Medidas integrales.

$2.4 \quad$ Referencias. 
La tesis se desarrolló empleando principalmente métodos ópticos in-situ en electroquímica además de técnicas convencionales, siguiendo con la línea de trabajo del grupo de investigación donde se han venido realizando diversos estudios en esta área durante varios años (Lezna, R.O. et al.,1986,1988a,1988b,1992,1994, 2009, Tacconi, N.R. et al.,2003, 2005, 2008, Juliarena, M. P. et al.,2006, Ruiz, G. T. et al.,2006, Wouters, K.L et al.,2006, Bracco, L.L.B., Muñoz Zúniga, J. et al.,2011, Shreeyukta, S. et al.,2011).

En el desarrollo de estos estudios se emplearon técnicas espectroelectroquímicas, UV-vis convencional y voltamperometrías (de corriente alterna (ACV) y de corriente continua (DCV)).

Dentro de las técnicas espectroelectroquímicas se utilizaron: espectroscopía diferencial (con modulación mínima de potencial), integral (modulación con amplitudes de potencial significativas), acompañando barridos lentos de potencial (estado "cuasi" estacionario, ca. $\mathrm{v} 1-3 \mathrm{mV} / \mathrm{s}$ ), medidas a potenciales fijos, y pulsos de potencial (espectros en función del tiempo con resolución temporal). También se empleó desconexión programada del control de potencial de la celda electroquímica (circuito abierto) manteniendo el monitoreo espectral, que hace posible verificar la existencia y/o el comportamiento de eventuales reacciones químicas (Bewick, A. et al.,1980, Lezna, R.O., Muñoz Zúniga J. et al.,2009). 


\subsection{Parte experimental}

Las medidas se realizaron a temperatura ambiente $\left(\sim 22^{\circ} \mathrm{C}\right)$ y las soluciones se prepararon con acetonitrilo (MeCN) - Biotech Grade 99,93\% de Aldrich. Como electrolito soporte se utilizó $0.1 \mathrm{M}$ hexafluorofosfato de tetrabutilamonio (TBAPF $)$ - Aldrich, el cual fue sometido a secado previo en una estufa de vacío a $60{ }^{\circ} \mathrm{C}$ durante $24 \mathrm{~h}$ aproximadamente.

Como electrodos de trabajo, se utilizaron discos de Pt u Au $(6 \mathrm{~mm}$ de diámetro) encapsulado en Kel-F o soldados en vidrio como muestra la figura 1. El electrodo de trabajo fue pulido mecánicamente con acabado especular, empleando $\mathrm{Al}_{2} \mathrm{O}_{3}$ de diferentes tamaños $(3.0 \mu \mathrm{m}, 1.0 \mu \mathrm{m}, 0.3 \mu \mathrm{m}, 0.05 \mu \mathrm{m}$ ); seguido de un baño ultrasónico para remover las partículas de alúmina de la superficie del electrodo. Cabe destacar que esta cuidadosa preparación de la superficie del electrodo y la limpieza de la solución, se hace necesaria con el fin de obtener medidas reproducibles y confiables (electroquímicas y ópticas) (Southampton Electrochemistry Group, 1985). El contraelectrodo utilizado fue una malla de $\mathrm{Pt}$, mientras que el electrodo de referencia fue $\mathrm{Ag} / \mathrm{AgCl}$ o $\mathrm{SCE}$, por lo tanto, todos los potenciales se midieron y están referidos respecto a estos electrodos. Para estudiar las reacciones que ocurren en la interfase (electrodo/solución) tanto en las medidas ópticas como electroquímicas, se utilizó una celda óptica cuyo diseño se muestra en la figura 2, y eventualmente para medidas electroquímicas se utilizó una celda convencional de tres electrodos, no óptica. 


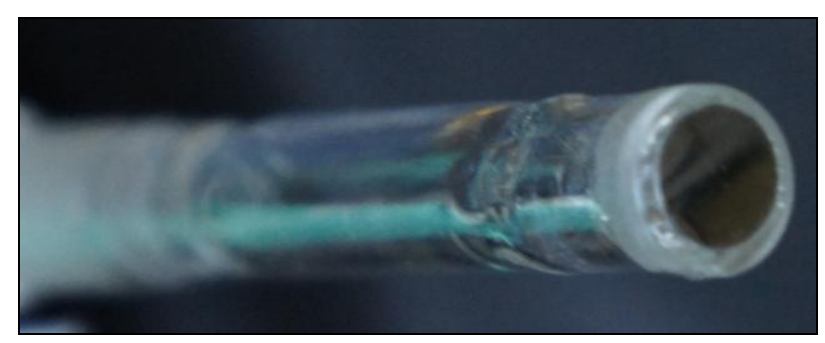

Figura 1. Electrodo de trabajo Pt

Antes y durante las medidas con solventes orgánicos se tuvo la precaución de mantener el sistema en general (electrolito soporte, complejo a utilizar, solvente y todo el material de vidrio) en un ambiente seco para disminuir la contaminación con agua.

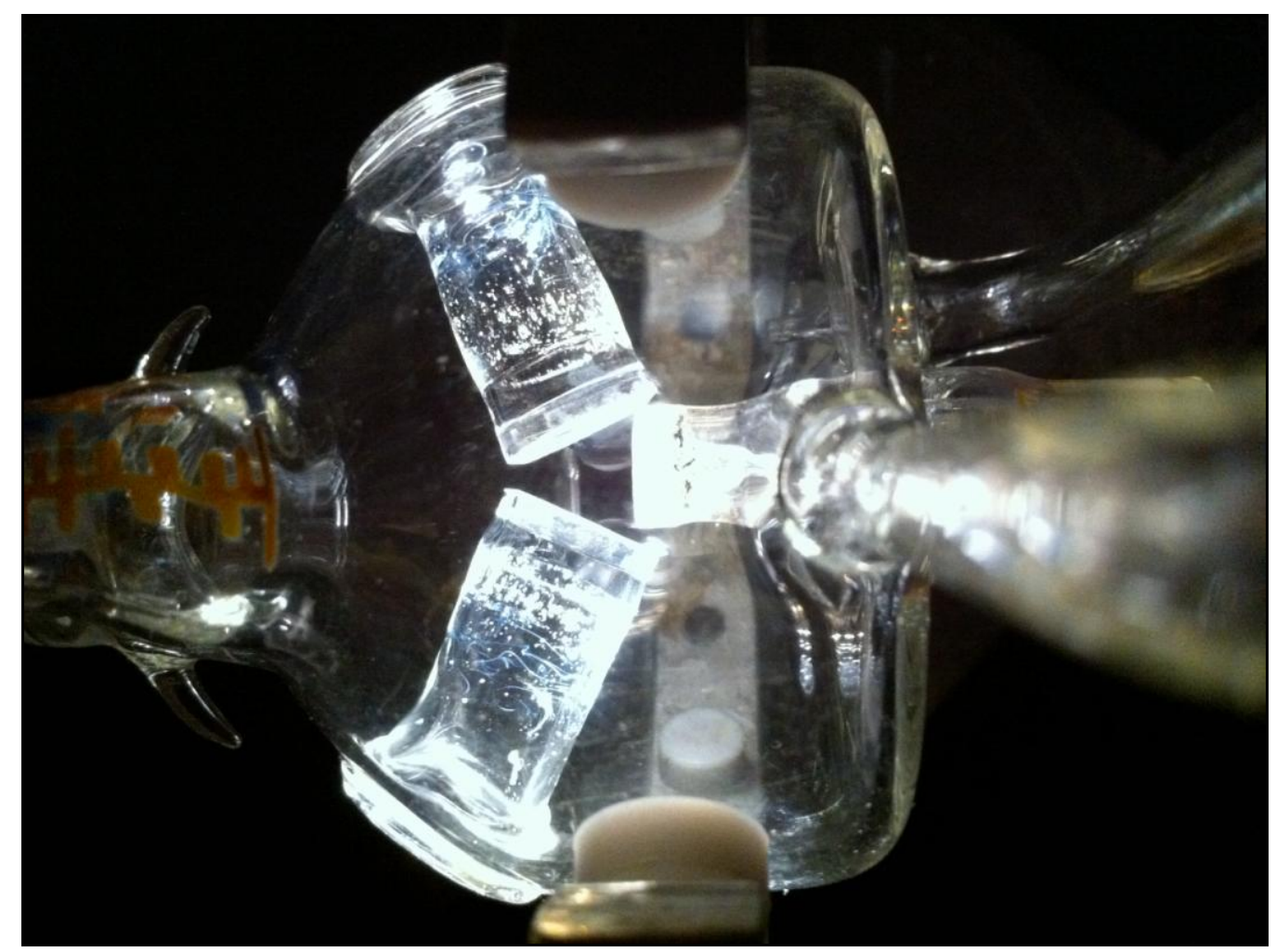

Figura 2. Celda electroquímica empleada en los estudios de ACV. 


\subsection{Medidas electroquímicas}

Las medidas electroquímicas convencionales se llevaron a cabo con un equipo computarizado con programas de control hechos en el laboratorio. El sistema está formado por un potenciostato PAR 173 con una interfaz IEEE-M276, un generador de formas de ondas y un osciloscopio Nicolet para almacenar respuestas en forma digital (Figura 3 ).

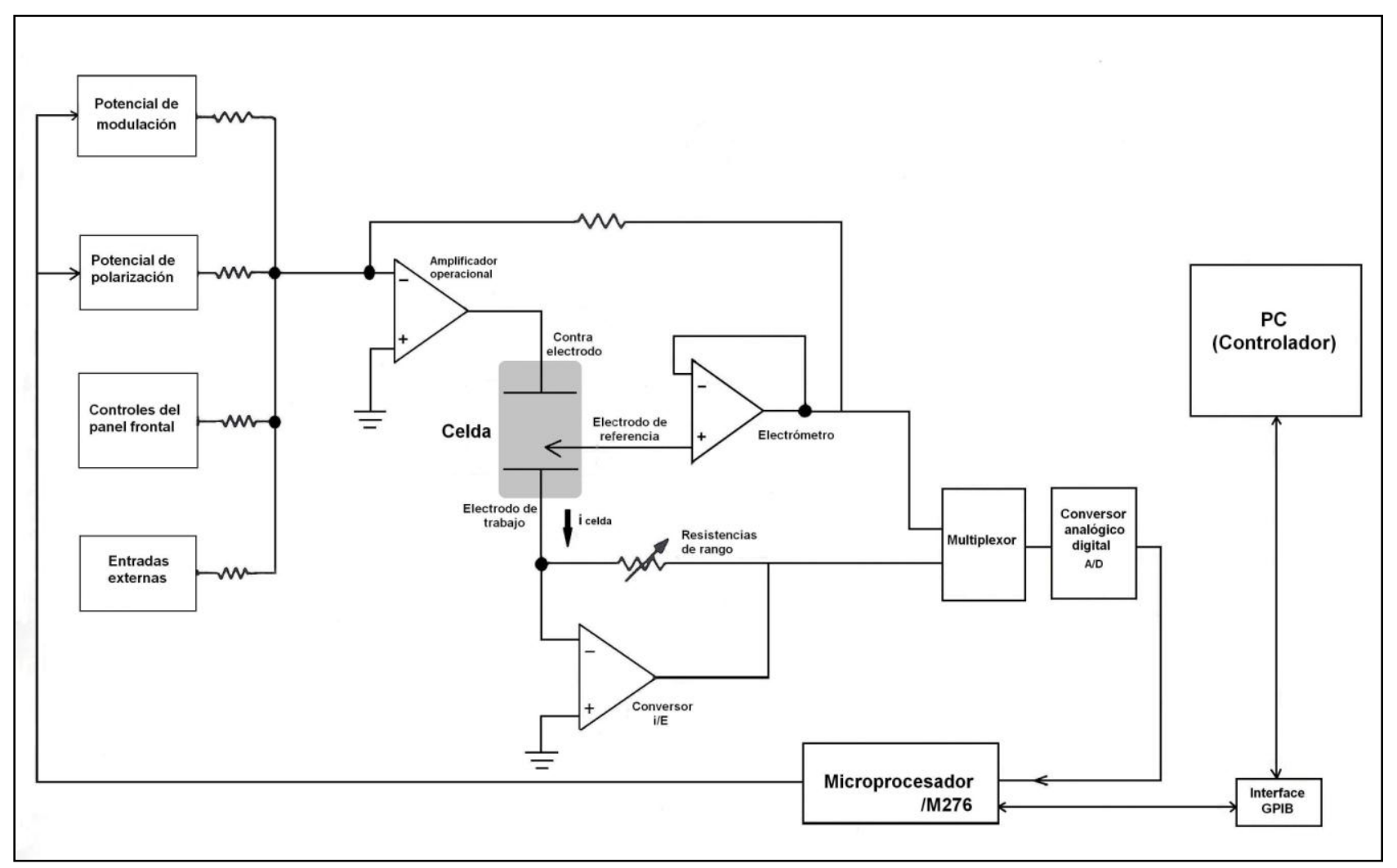

Figura 3. Sistema utilizado para medidas electroquímicas convencionales.

La unidad M276, permite interfaciar el potenciostato con el resto de los equipos que se encuentren sobre la interfaz GPIB (IEEE488). Consta de un microprocesador, memorias y programas internos que le permiten actuar como 
controlador de experimentos incluyendo un generador de funciones. Tiene también la capacidad de almacenar internamente datos los cuales posteriormente pueden ser transferidos al controlador principal (PC).

\subsubsection{La doble capa eléctrica}

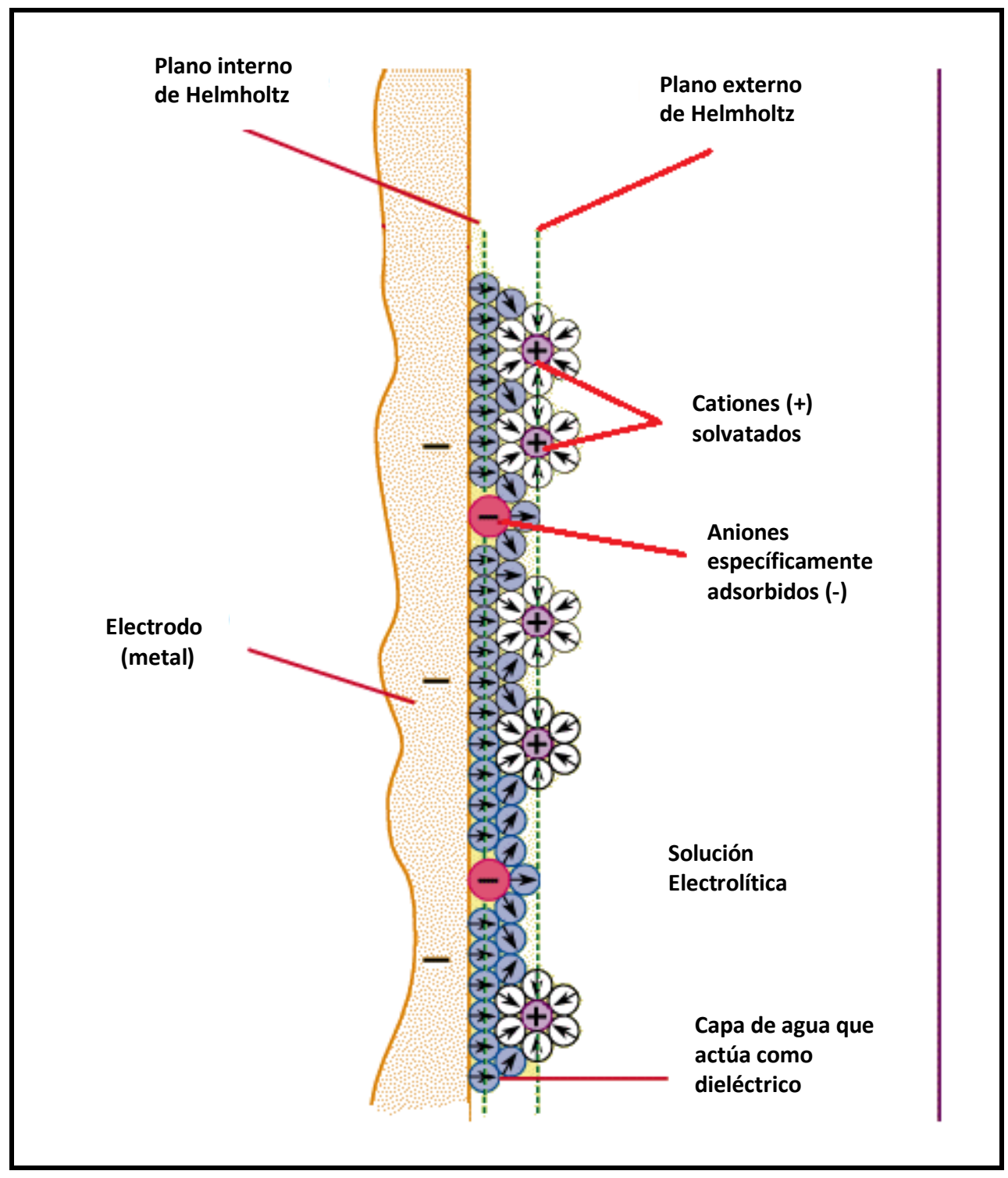

Figura 4. Doble capa eléctrica. 
La doble capa es un término que describe la configuración, integralmente neutra, que presentan los iones y moléculas en la interfase electrodo-solución que puede ser conductora como en nuestro caso (electrodo metálico), o semiconductora. La existencia de la doble capa se debe a la acumulación de carga en las dos fases interfaciadas que da lugar a un comportamiento capacitivo.

En sistemas electroquímicos, en la fase del electrodo (conductor metálico) los electrones libres responden al campo producido por la acumulación de carga en la región de la interfase. De esta manera se induce una carga de signo opuesto e igual magnitud a la fase electrolito (de la interfase), produciendo una separación de cargas en la interfase electrodo/electrolito (Figura 4). Sin embargo la región total a la cual llamamos interfase es eléctricamente neutra. Cuando se separan las cargas, se genera una diferencia de potencial a través de la interfase.

Las cargas eléctricas que operan entre el metal y la solución constituyen el campo eléctrico a través del límite electrodo-electrolito. Aún cuando la diferencia de potencial a través de la interfase no es grande $(\sim 1 \mathrm{~V})$, las dimensiones de la región que constituye la interfase son muy pequeñas $(\sim 10 \AA)$ y, por lo tanto, la fuerza del campo (gradiente de potencial) es enorme, del orden de $10^{7} \mathrm{~V} \mathrm{~cm}^{-1}$ (Bockris, J.O'M et al.,2006).

\subsubsection{Cinética electroquímica}

Se considera la reacción 1, asumiendo una solución que inicialmente contiene solamente la especie $\mathrm{O}$, en un experimento en el que al comienzo no fluye corriente 


$$
O+z e^{-} \Leftrightarrow R
$$

Como se supone que la cinética de transferencia de carga es muy rápida, según la ecuación de Nernst, tenemos:

$$
E=E^{0^{\prime}}+\frac{R T}{z F} \ln \frac{C_{O}(0, t)}{C_{R}(0, t)}
$$

Donde,

$E^{\circ} \quad$ : Potencial en condición estándar.

$C_{o}$ : Concentración de la especie $\mathrm{O}$ en la interfase electrodo/ solución.

$C_{R} \quad$ : Concentración de la especie $\mathrm{R}$ en la interfase electrodo/ solución.

$F \quad$ : Constante de Faraday.

$R \quad$ : Constante de los gases.

$T$ : Temperatura (en ${ }^{\circ} \mathrm{K}$ ).

$z \quad$ : Número de electrones intercambiados en la reacción.

$E^{\circ}$, es el potencial cuando las actividades de $\mathrm{O}$ y $\mathrm{R}$ en la interfase del electrodo son iguales a la unidad. Al principio de la medida las concentraciones de $\mathrm{O}$ y $\mathrm{R}$ son iguales en la interfase del electrodo y en el seno de la solución, de aquí que se puede escribir:

$$
\begin{gathered}
\mathrm{E}_{\mathrm{celda}}=\mathrm{E}_{\mathrm{WE}}-\mathrm{E}_{\mathrm{NHE}}=\mathrm{E}_{\mathrm{WE}}-0 \\
E=E^{0^{\prime}}+\frac{R T}{z F} \ln \frac{C_{O}^{*}(0, t)}{C_{R}^{*}(0, t)}
\end{gathered}
$$

Donde,

$C_{O}^{*} \quad$ : Concentración de la especie $\mathrm{O}$ en la solución.

$C_{R}^{*} \quad$ : Concentración de la especie $\mathrm{R}$ en la solución. 
Aunque no fluya una corriente neta y no haya cambios químicos en la celda, existe un equilibrio dinámico en la superficie del electrodo de trabajo, es decir ocurre tanto la reducción de $\mathrm{O}$ como la oxidación de $\mathrm{R}$, pero los procesos son de igual velocidad y opuestos:

$$
-\vec{j}=\overleftarrow{j}=j_{0} \quad\left(\text { referida también como } i_{0}\right)
$$

Donde $j_{0}$ es una importante característica cinética en un proceso de transferencia electrónica conocida como densidad de corriente de intercambio. $\vec{j}$ y $\underset{j}{ }$ son las densidades de corriente de intercambio parciales para las reacciones directa e inversa. El signo negativo convencionalmente es para indicar la corriente catódica.

Si el potencial aplicado al electrodo de trabajo se hace más negativo que el potencial de equilibrio determinado por las concentraciones de $O$ y $R$ en la interfase, las concentraciones de $\mathrm{O}$ y $\mathrm{R}$ evolucionan hacia el nuevo valor exigido por la ecuación de Nernst (Ec. 1). Para ello es necesario que fluya una corriente neta a través de la interfase electrodo/solución. De hecho, es necesaria una disminución en la relación $\mathrm{C}_{0} / \mathrm{C}_{\mathrm{R}}$ y esto puede llevarse a cabo por el paso de corriente catódica que convierte $\mathrm{O}$ en $\mathrm{R}$. Por el contrario, si el potencial aplicado al electrodo de trabajo es más positivo que el potencial de equilibrio $\mathrm{E}$, se observa una corriente anódica.

Estas predicciones sencillas sobre la relación $I-E$ en una celda electroquímica, se basan únicamente en la termodinámica (equilibrio, sin limitaciones cinéticas) sin embargo en sistemas reales, la magnitud de la corriente que fluye a cualquier potencial dependerá también de la cinética de transferencia de electrones a un potencial dado:

$$
j=\vec{j}+\overleftarrow{j}
$$


(Donde $\vec{j}$ tiene signo negativo). Estas densidades de corrientes parciales son cada una dependiente de la constante de velocidad $(k)$ y de la concentración de las especies electroactivas en el sitio de transferencia de electrones (electrodo).

$$
\overleftarrow{j}=z F k C_{R} \quad \text { y } \quad \vec{j}=-z F k C_{O}
$$

Sin embargo, la constante de velocidad tiene, en este caso, una propiedad particular, varía con el potencial aplicado al electrodo (es decir, varía con la diferencia de potencial en la interfase -electrodo/solución- durante la transferencia de electrones) de acuerdo a la siguiente relación:

$$
\vec{k}=\vec{k}_{0} \exp \left(-\frac{\alpha_{C} F}{R T} E\right) \text { y } \quad \stackrel{\leftarrow}{k}=\overleftarrow{k}_{0} \exp \left(\frac{\alpha_{A} F}{R T} E\right) \quad \text { Ec. } 7
$$

donde, $\alpha_{A}$ y $\alpha_{C}$ son constantes conocidas como coeficientes de transferencia de la reacción anódica y catódica respectivamente.

Operando algebraicamente las ecuaciones 5,6,7 y definiendo el sobrepotencial como la desviación del potencial del valor de equilibrio:

$$
\eta=E-E_{e}
$$

y la densidad de corriente de intercambio como $j_{0}=-\vec{j}=\overleftarrow{j}$ a $\eta=0$, se tiene la ecuación de Butler - Volmer:

$$
j=j_{o}\left[\exp \left(\frac{\alpha_{A} F}{R T} \eta\right)-\exp \left(-\frac{\alpha_{C} F}{R T} \eta\right)\right]
$$

donde,

j : : Densidad de corriente. 
$\eta \quad$ : Sobrepotencial.

$\alpha_{A}, \alpha_{C}$ : Coeficientes de transferencia de la reacción anódica y catódica respectivamente.

Hay tres simplificaciones para la ecuación 9, las primeras dos ocurren a altos sobrepotenciales. En sobrepotenciales altamente positivos $|\overleftarrow{j}|>>|\vec{j}|$, el segundo término puede ser ignorado, y la densidad de corriente anódica está dada por:

$$
\log j=\log j_{0}+\frac{\alpha_{A} F}{2.3 R T} \eta
$$

por el contrario, a sobrepotenciales altamente negativos $|\vec{j}|>>|\overleftarrow{j}|$, la densidad de corriente catódica está dada por:

$$
\log j=\log j_{0}-\frac{\alpha_{C} F}{2.3 R T} \eta
$$

La tercera simplificación se aplica a muy bajos valores de $\eta$ donde $\eta<<$ $\left(\frac{R T}{\alpha_{C} F}\right)$ y $\eta<<\left(\frac{R T}{\alpha_{A} F}\right)$. Los dos términos exponenciales de la ecuación 9 , pueden ser desarrollados en serie, y despreciando los términos de orden superior la ecuación se reduce a la forma lineal:

$$
j=j_{0} \frac{F}{R T} \eta
$$

La ecuación 9, debe ser considerada como una ecuación fundamental en la cinética de los electrodos ya que muestra la forma en que la densidad de corriente varía con la densidad de corriente de intercambio, el sobrepotencial y los coeficientes de transferencia (Southampton Electrochemistry Group,1985, Bockris, J.O'M et al.,2006). 


\subsubsection{Determinación de la cinética de electrodos}

Para medir los parámetros cinéticos de los procesos de transferencia de electrones como, por ejemplo: la constante de velocidad heterogénea, $\mathrm{k}$, el coeficiente de transferencia de reacción (catódica o anódica), $\alpha$, entre otros, se hace necesario estudiar el mecanismo del proceso electroquímico de un sistema, el cual, en general, incluye 3 pasos sucesivos:

1. La difusión del reactivo a la interfase electrodo/solución.

2. Transferencia heterogénea de electrones.

3. La difusión de los productos hacia el seno de la solución.

La cinética resultante de esos procesos, que incluyen varios pasos consecutivos, está determinada por el paso más lento cuya velocidad es lo que se mide experimentalmente.

En la Figura 5 se puede ver un modelo simplificado de un circuito equivalente correspondiente a la interfase electrodo/solución.

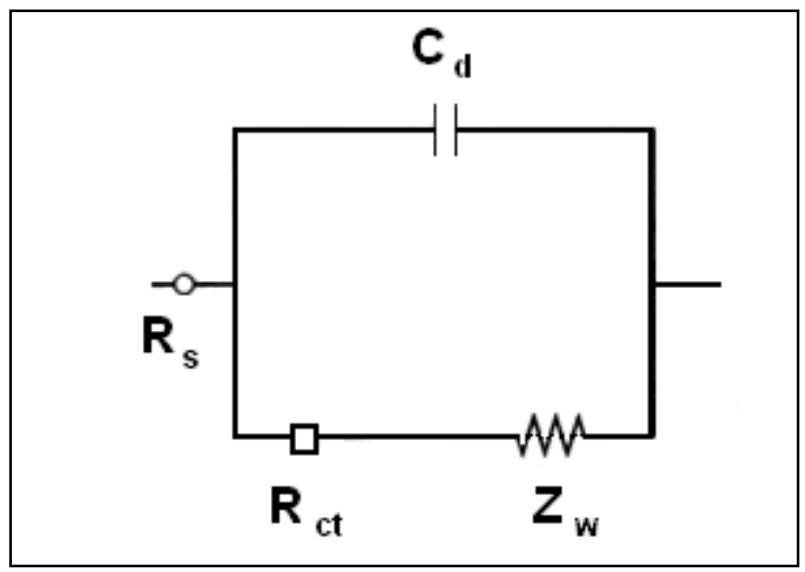

Figura 5. Circuito equivalente correspondiente a la interfase electrodo/solución. 
donde,

$\mathrm{R}_{\mathrm{s}}$ : resistencia de la solución (óhmica)

$\mathrm{R}_{\mathrm{ct}}$ : resistencia de transferencia de carga

$Z_{\mathrm{w}}$ : impedancia de Warburg

$\mathrm{C}_{d}$ : doble capa (capacitancia)

Los electrones son transferidos a través de la interfase vía dos caminos paralelos:

1. El proceso faradaico representado por $R_{c t} y Z_{w}$ conectados en serie.

2. La carga/descarga de la doble capa eléctrica.

En ambos casos, el flujo de corriente atraviesa la solución, cuya resistencia óhmica está representada por $R_{\mathrm{s}}$ (sin compensación). El segundo camino es importante solamente si el potencial del electrodo es cambiado rápidamente (perturbación rápida o transitorio) a altas frecuencias, siendo relevante en técnicas como cronoamperometría, pulsos de potencial a tiempos cortos, etc.

\subsubsection{Pulsos de potencial}

En experimentos de pulsos, el potencial del electrodo de trabajo es cambiado rápidamente registrándose la respuesta en la forma de corriente tiempo o carga - tiempo.

Considerando la reacción 1, para una perturbación potencial - tiempo como la mostrada en la figura 6 , se elige $E_{1}$, de modo que no haya reducción de la especie $O$. Luego en el momento $t=0$, el potencial es rápidamente cambiado a un 
nuevo valor $\mathrm{E}_{2}$, donde la reducción de $\mathrm{O}$ ocurre a una velocidad controlada por difusión.

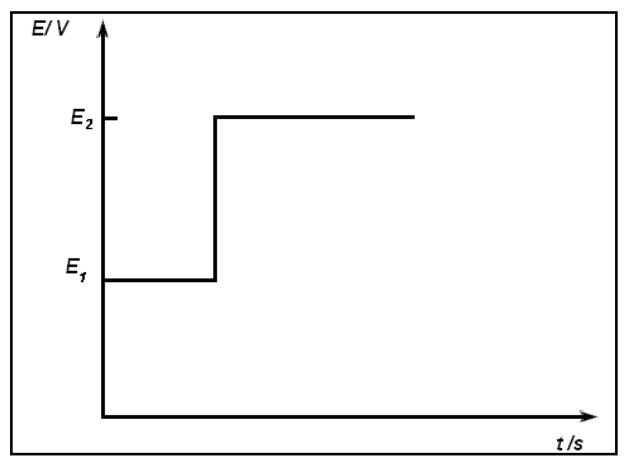

Figura 6. Perfil potencial-tiempo para un pulso de potencial.

Considerando una cinética rápida de transferencia de carga, para la reacción de la ecuación 1 , se puede deducir también que las ecuaciones que gobiernan en este caso la transferencia de masa, son las leyes de Fick, ecuaciones $13,14,15$.

$$
\frac{\partial C_{O}(x, t)}{\partial t}=D_{O} \frac{\partial^{2} C_{O}(x, t)}{\partial x^{2}} \quad \frac{\partial C_{R}(x, t)}{\partial t}=D_{R} \frac{\partial^{2} C_{R}(x, t)}{\partial x^{2}}
$$

Donde $D_{O}$ y $D_{R}$ corresponden a los coeficientes de difusión de las especies O y $\mathrm{R}$ respectivamente.

Las condiciones iniciales son:

1. Homogeneidad en la solución antes de iniciar la medida a $t=0$, ecuación 14.

$$
\begin{array}{lll}
C_{O}(x, 0)=C_{O}^{*} & C_{R}(x, 0)=0 & \text { Ec. } 14
\end{array}
$$

2. Condición de difusión semi-infinita (Ec. 15): se supone que en el experimento, las regiones alejadas de los electrodos no son perturbadas. 


$$
\lim _{x \rightarrow \infty} C_{O}(x, 0)=C_{O}^{*}
$$

$\lim _{x \rightarrow \infty} C_{R}(x, 0)=0$

Ec. 15

3. La ecuación 16 describe la condición de contorno en la interfase (electrodo/ solución), después de la aplicación del potencial.

$$
C_{O}(0, t)=0 \quad(\text { para } t>0)
$$

Ec. 16

Utilizando cálculos matemáticos para resolver las ecuaciones de Fick teniendo en cuenta las condiciones de contorno, se llega a la relación I-t (transitorio), ecuación 17, denominada ecuación de Cottrell, la cual rige en condiciones de control difusional (Bard, A. J. y Faulkner, L. R. 2001).

$$
I(t)=I_{d}(t)=\frac{z A F D_{o}^{1 / 2} C_{O}^{*}}{(\pi t)^{1 / 2}}
$$

Donde $I_{d}$ corresponde a la corriente de difusión, A al área del electrodo y $t$ al tiempo.

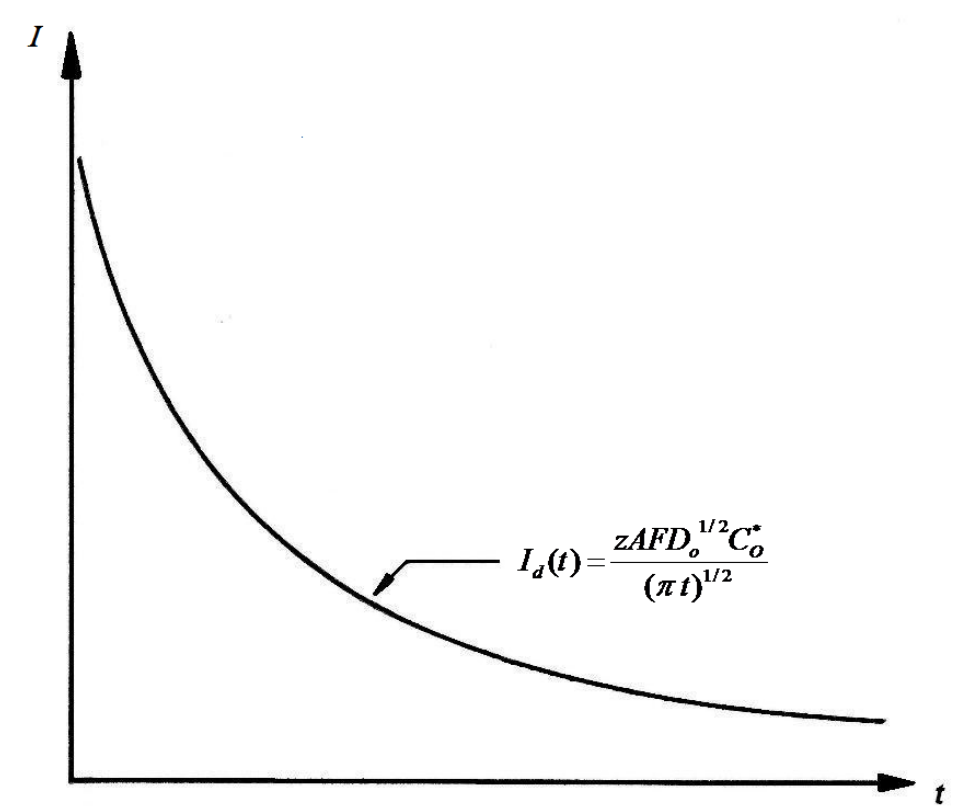

Figura 7. Respuesta de un proceso difusional a un pulso de potencial 
La respuesta típica de corriente vs tiempo para procesos difusionales se observa en la figura 7 (Southampton Electrochemistry Group, 1985 ).

Utilizando pulsos de potencial, se pueden realizar análisis en diferentes sistemas que incluyen además del caso descripto (proceso difusional), estudios cinéticos, de adsorción, etc. Esta técnica también puede ser usada para caracterizar procesos de electrocristalización (formación de nuevas fases).

\subsubsection{Voltamperometría}

La voltamperometría es un conjunto de técnicas transitorias, basadas en la relación que existe entre corriente y potencial en un proceso electroquímico (Harris, D.C., 1992). Esta técnica se ha convertido en un procedimiento esencial para los estudios electroquímicos iniciales de nuevos sistemas y ha demostrado ser muy útil para obtener información acerca de reacciones en la interfase y en solución (Bard, A. J. y Faulkner, L. R. 2001). En el desarrollo de esta tesis se llevaron a cabo medidas voltamperométricas DCV y ACV.

\subsubsection{Voltamperometría de corriente continua - DCV}

La voltamperometría cíclica (CV), es una técnica usada para estudiar mecanismos de reacciones electroquímicas. El método consiste en aplicar (al electrodo de trabajo) una variación lineal de potencial a una velocidad específica y medir la corriente resultante. La respuesta, conocida como voltamperograma, 
proporciona en general una buena información cualitativa y cuantitativa sobre el mecanismo de reacción.

Consideramos nuevamente la reacción $1 O+z e^{-} \Leftrightarrow R$ asumiendo difusión lineal semi-infinita, teniendo al inicio solamente la especie $\mathrm{O}$ y al electrodo en un potencial $E_{i}$, donde no se producen reacciones (condiciones iniciales similares a las empleadas para la técnica de pulsos de potencial).

El potencial se barre linealmente a una velocidad $v,(\mathrm{~V} / \mathrm{s})$, de modo que en cualquier momento $t$, estaría dado por la ecuación 18.

$$
E=E_{i}-v t, \quad 0<t<\lambda
$$

Donde, $\lambda$ corresponde al tiempo donde ocurre la inversión del barrido. Una vez que el potencial alcanza el valor máximo $E_{\lambda}$ se invierte el barrido y el potencial retorna al valor inicial $E_{i}$ figura 8 . En el barrido de retorno, el potencial viene dado por la ecuación 19.

$$
E=E_{\lambda}-2 v \lambda+v t, \quad t>\lambda
$$

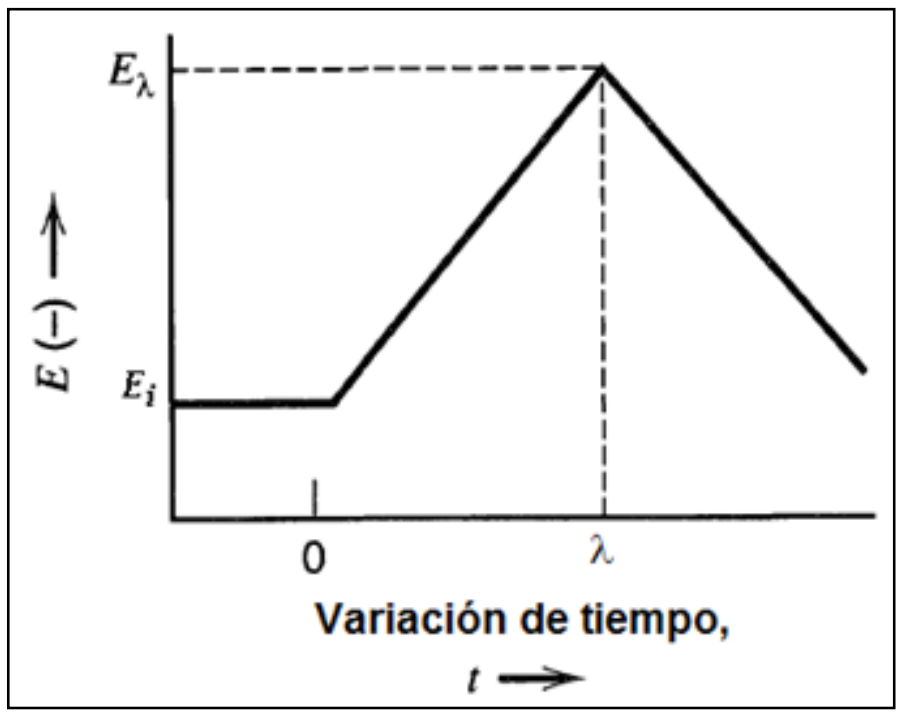

Figura 8. Barrido lineal de potencial para CV. 
Si se asume que la velocidad de transferencia de electrones en la interfase electrodo/solución, es rápida, las concentraciones de las especies $\mathrm{O}$ y $\mathrm{R}$ se ajustan inmediatamente a la relación dada por la ecuación de Nernst (Ec. 1), y siguen siendo válidas las ecuaciones 13,14 y 15.

Resolviendo la ecuación de Fick, se llega a la ecuación matemática que cuantifica la corriente máxima (o intensidad máxima) en un sistema reversible dada por la ecuación de Randles-Sevčik, (a $25^{\circ}$ C), ecuación 20

$$
I_{p}=\left(2.69 \times 10^{5}\right) z^{3 / 2} A C_{O}^{*} D_{o}^{1 / 2} v^{1 / 2}
$$

Donde $I_{p}$ es la corriente de pico, D es el coeficiente de difusión en $\mathrm{cm}^{2} \cdot \mathrm{s}^{-1}, v$ es la velocidad de barrido en $\mathrm{V}^{-\mathrm{S}^{-1}}$ y $\mathrm{C}^{*}$ o es la concentración de la especie $\mathrm{O}$ en mol. $\mathrm{cm}^{-3}$. Como podemos ver la corriente de pico es proporcional a la concentración de la especie electroactiva, a la raíz cuadrada de la velocidad de barrido y al coeficiente de difusión.

En la figura 9 se muestra un voltamperograma como respuesta a la aplicación de un barrido lineal de potencial a una velocidad constante (por ejemplo, $50 \mathrm{mV} / \mathrm{s}$; entre $+100 \mathrm{mV}$ y $-1500 \mathrm{mV}$ (ida y vuelta)). Para este caso específico (figura 9) se analiza el comportamiento electroquímico de la especie $\mathbf{0}$; la medida se inicia a un potencial más positivo que $\mathrm{E}^{\mathrm{o}^{\prime}}\left(\mathrm{E}_{\mathrm{i}}\right)$. Antes de la reducción de $\mathbf{O}$, se registra en el barrido (como transitorio) solamente el flujo de corriente no faradaica (capacitiva). Cuando el potencial llega a valores alrededor de $E^{o^{\prime}}$, se inicia el flujo de electrones desde el electrodo hacia la solución debido a la reducción de $\mathbf{O}$ para dar $\mathbf{R}$ (corriente faradaica). A medida que el potencial sigue creciendo negativamente, la corriente (velocidad de reducción) aumenta y esto genera disminución de la concentración de la especie $\mathbf{O}$ en la interfase (electrodo/solución). 
A medida que el potencial va sobrepasando $\mathrm{E}^{\mathrm{o}^{\prime}}$, la concentración de $\mathbf{O}$ en la interfase cae a valores cercanos a cero y la reacción aumenta su velocidad. La cinética de este proceso es dominada por la difusión de $\mathbf{O}$ desde el seno de la solución a la superficie del electrodo llegando a un valor máximo de corriente, el cual luego decae debido al agotamiento de la concentración de $\mathbf{O}$ en la interfase.

Si se invierte la dirección del barrido de potencial, desde $E_{\lambda}$ hasta $E_{i}$, se obtiene el proceso de oxidación donde se forma la especie $\mathrm{O}$ y se consume la especie $R$.

La figura 9, describe el resultado de la presencia de dos procesos que generan resultados inversos, el aumento de potencial (que aumenta la corriente) y la disminución de concentración (que disminuye la corriente) en la superficie del electrodo, como consecuencia la corriente pasa por un máximo.

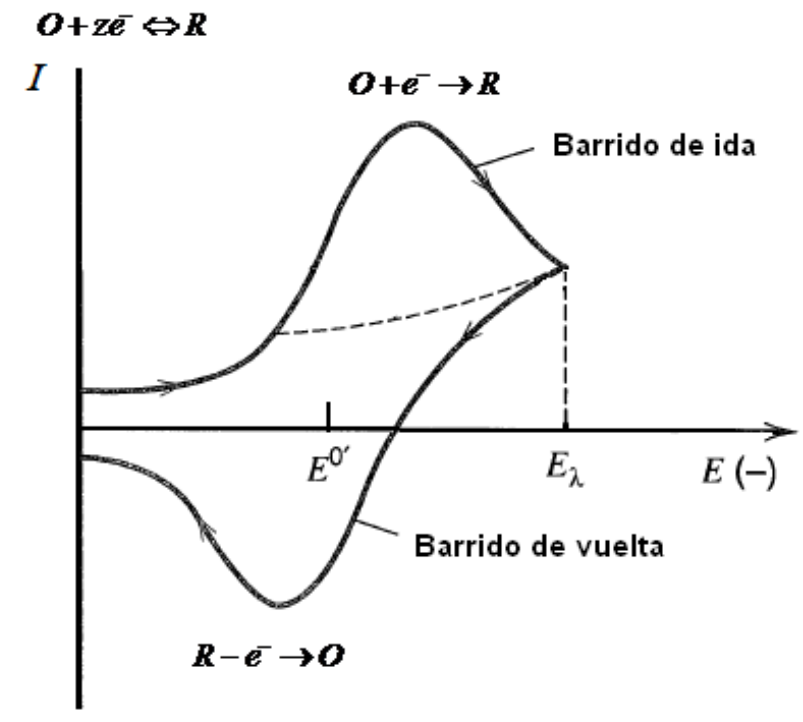

Figura 9. Voltamperograma para un sistema reversible tipo $O+z e^{-} \Leftrightarrow R$ 
En el caso en el que se aplica un barrido lineal de potencial con una velocidad muy lenta, i.e. $1 \mathrm{mV} / \mathrm{s}$ a un sistema reversible como el de la reacción 1 (primer barrido de la figura 10), el voltamperograma resultante es similar al que se obtiene en estado estacionario (por medidas punto a punto). Es decir los fenómenos que ocurren en la interfase son independientes del tiempo aunque exista flujo de una corriente neta indicando actividad a lo largo de este proceso. Las concentraciones de los productos intermediarios de la reacción permanecen constantes en el transcurso del tiempo, la carga que penetra en la interfase es igual a la que sale de la misma. De esta forma no varía la respuesta de la interfase, ni la diferencia de potencial a través de la misma (Bockris, J.O'M et al.,2006). Sin embargo, al aumentar la velocidad de barrido la curva evoluciona hacia la de un transitorio observándose un incremento en la corriente como se muestra en la figura 10. Aparece un máximo de corriente que se hace más prominente a partir de los efectos combinados de mayor velocidad de transferencia de carga y agotamiento progresivo de $O$ (de la reacción 1) en la capa difusional (Southampton Electrochemistry Group, 1985).

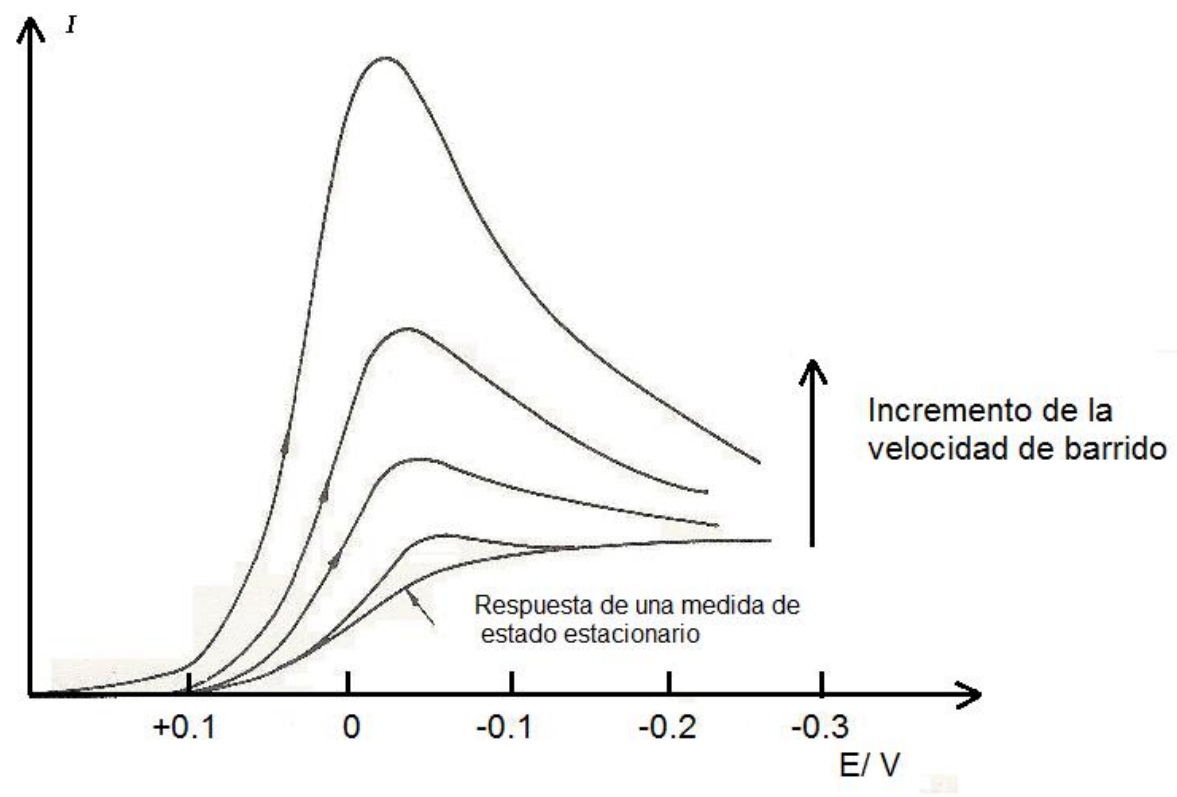

Figura 10. Serie de voltamperogramas de barrido lineal para una reacción tipo $O+z e^{-} \Leftrightarrow R$, utilizando varias velocidades de barrido. 
Mediante DCV también se estudia el comportamiento de sustancias adsorbidas sobre electrodos sólidos, este tema resulta importante debido a la relevancia tecnológica de las especies adsorbidas. Esto es especialmente cierto para metales nobles empleados como electrodos 0 como partes de electrocatalizadores en celdas de combustible y otras aplicaciones (Bard, A. J. y Faulkner, L. R. 2001). Un ejemplo clásico de sistema cuyo resultado muestra fuerte electroadsorción es la respuesta de un electrodo de Pt policristalino en solución acuosa ácida; la curva voltamperométrica corriente - potencial muestra picos correspondientes a la formación y remoción tanto de hidrógeno como de oxígeno adsorbidos (Figura 11) (Southampton Electrochemistry Group, 1985).

En la figura 11 se presenta un voltamperograma cíclico utilizando un electrodo de $\mathrm{Pt}$ en $\mathrm{H}_{2} \mathrm{SO}_{4}$ 0,5 M. La región 1 corresponde a la zona previa al desprendimiento de hidrógeno molecular; hacia potenciales más positivos se observan dos picos, con comportamiento reversible, denominados $\mathrm{H}_{\mathrm{f}}$ hidrógeno fuertemente adsorbido y $H_{d}$ hidrógeno débilmente adsorbido. La expresión (A) y (C) en la nomenclatura de los picos, indican la oxidación (anódica, A) y la formación (catódica, $\mathrm{C}$ ) del hidrógeno adsorbido respectivamente. En la región 2 se muestra la zona previa al desprendimiento de oxígeno, la reacción de formación de óxidos es compleja (no puramente electroquímica) y muestra un comportamiento irreversible. Los picos $\mathrm{O}_{(A)}$ y $\mathrm{O}_{(\mathrm{C})}$ indican la formación de una capa de oxido de Pt y la reducción de la misma respectivamente. Estas dos regiones, de hidrógeno y oxígeno, son ejemplos de deposición a subpotenciales, (UPD).

La respuesta presente en la región intermedia, entre el UPD de hidrógeno y el de oxígeno, corresponde a la zona de carga de la doble capa y es por lo tanto una corriente capacitiva. 


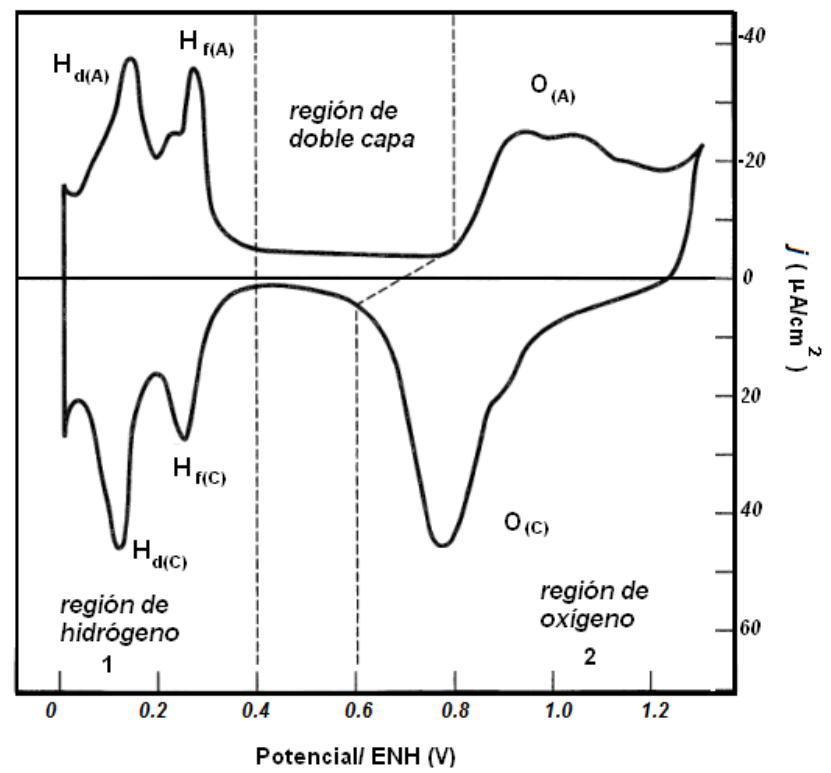

Figura 11. Voltamperograma cíclico utilizando un electrodo de Pt en $\mathrm{H}_{2} \mathrm{SO}_{4}$.

La forma, el número y tamaño de los picos de hidrógeno adsorbido dependen de las caras cristalinas expuestas del Pt policristalino, el pretratamiento del electrodo, las impurezas, si las hubiere, de la solución y el electrolito soporte (Bard, A. J. y Faulkner, L. R. 2001).

\subsubsection{Voltamperometría de corriente alterna ACV}

Las técnicas de corriente alterna $A C$, poseen una amplia aplicación en diversos campos de la electroquímica, e.g. electroanalítica, espectroscopía de impedancia, estudios de corrosión y en voltamperometría (William T. Y. et al., 1998). 
La voltamperometría de corriente alterna consiste en la aplicación de un potencial sinusoidal de amplitud pequeña $<10 \mathrm{mV}_{\mathrm{p}-\mathrm{p}}$, como perturbación a un sistema electroquímico, la respuesta del mismo, en corriente, también de forma sinusoidal (aunque atenuada y desfasada), es analizada con un detector sincrónico ((LIA) lock-in amplifier) o analizador de frecuencias que aumenta notablemente la sensibilidad de la respuesta que se observa como una señal rectificada (continua). ACV también revela información mecánistica y cinética que no es fácilmente accesible por técnicas electroquímicas tradicionales (por ejemplo: estacionarias).

La corriente alterna se mide a diferentes valores de potencial estacionario los que se pueden aplicar manualmente o a través de un barrido lento de potencial ( $v$ menor de ca. $2 \circ 3 \mathrm{mV} / \mathrm{s}$ ). La perturbación aplicada es de la forma señalada en la ecuación 21 y se representa en la figura 12.

$$
u(t)=A_{o} \operatorname{sen}(\omega t+\beta)
$$

Donde,

$u(t) \quad$ : Potencial sinusoidal aplicado.

$\mathrm{A}_{0} \quad$ : Amplitud (mV)

$\omega \quad$ : Frecuencia (rad/s).

$\beta \quad$ : Ángulo de fase inicial.

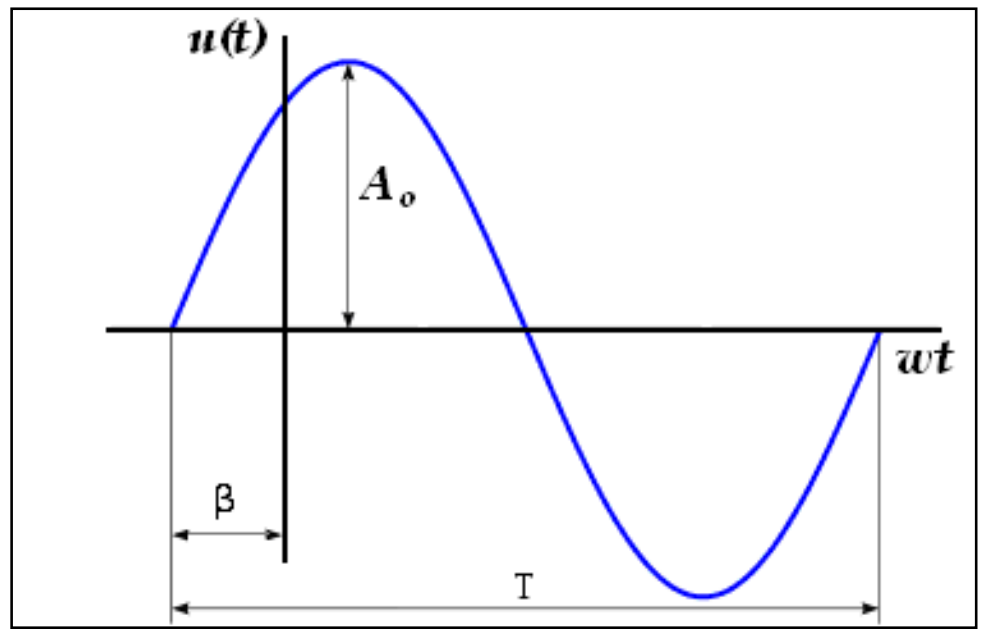

Figura 12. Perturbación sinusoidal 
La respuesta generada en ACV, se muestra en la figura 13

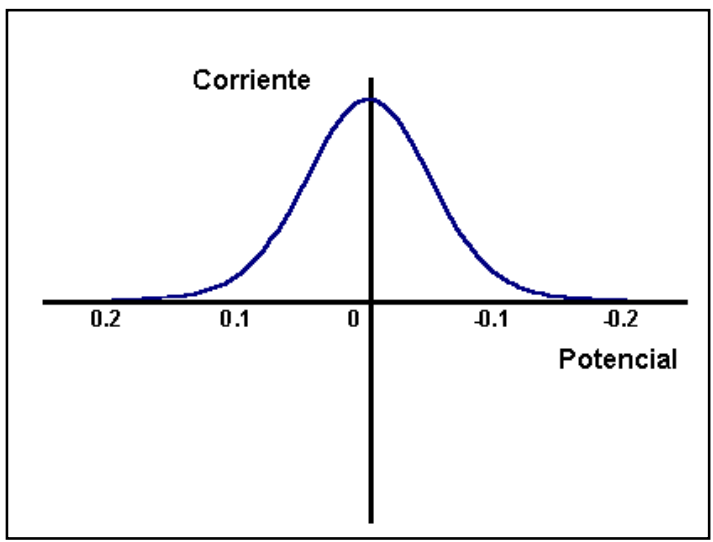

Figura 13. Respuesta corriente vs potencial en ACV después de pasar por el LIA.

Un detalle importante de la Figura 12 es la magnitud de la perturbación $\mathrm{AC}$, la cual típicamente debe tener una amplitud siempre menor que $10 \mathrm{mV}_{\mathrm{p}-\mathrm{p}}$ para asegurar linealidad en la respuesta en este tipo de medidas.

Esta pequeña perturbación asegura sólo variaciones leves en las concentraciones cerca de la interfase (electrodo/solución) que conduce a resultados lineales,(como sugiere la ecuación 12) a pesar de que la cinética de los electrodos tiene una dependencia exponencial con el potencial aplicado (observable a mayores amplitudes).

Utilizando voltametría de corriente alterna se pueden estudiar diferentes tipos de sistemas con la condición de que sean suficientemente reversibles como para seguir la modulación sin degradarse. Un ejemplo de sistema reversible, se puede observar en la figura 14, correspondiente a una medida de ACV de ferroceno en MeCN (realizada en nuestro laboratorio), 


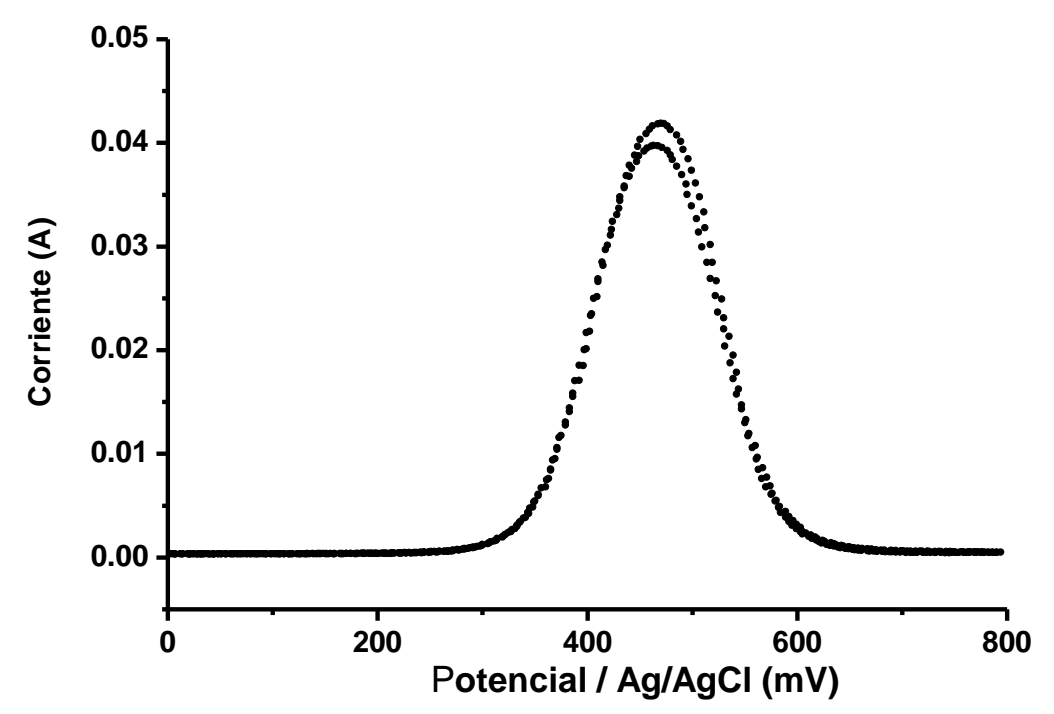

Figura 14. Medida $\mathrm{ACV}$ de ferroceno en $\mathrm{MeCN}, 6 \mathrm{~Hz}$ de frecuencia, utilizando Pt como electrodo de trabajo.

Las mediciones voltamperométricas AC realizadas en esta tesis para los diferentes sistemas analizados, se llevaron a cabo con una pequeña onda sinusoidad generalmente de $5 \mathrm{mV}$ pico a pico de amplitud sumada a una velocidad de barrido lineal muy lenta de aproximadamente $5 \mathrm{mV} / \mathrm{s}$ para realizar la medida en varios potenciales. La corriente, respuesta de la interfase electroquímica a la perturbación sinusoidal, se analiza con un detector sincrónico (lock-in amplifier) que la rectifica para dar una señal de continua en función del potencial, ver e.g. (Lezna, R.O. et al.,1988a, 2009). Este sistema de medida se representa en la Figura 15. 


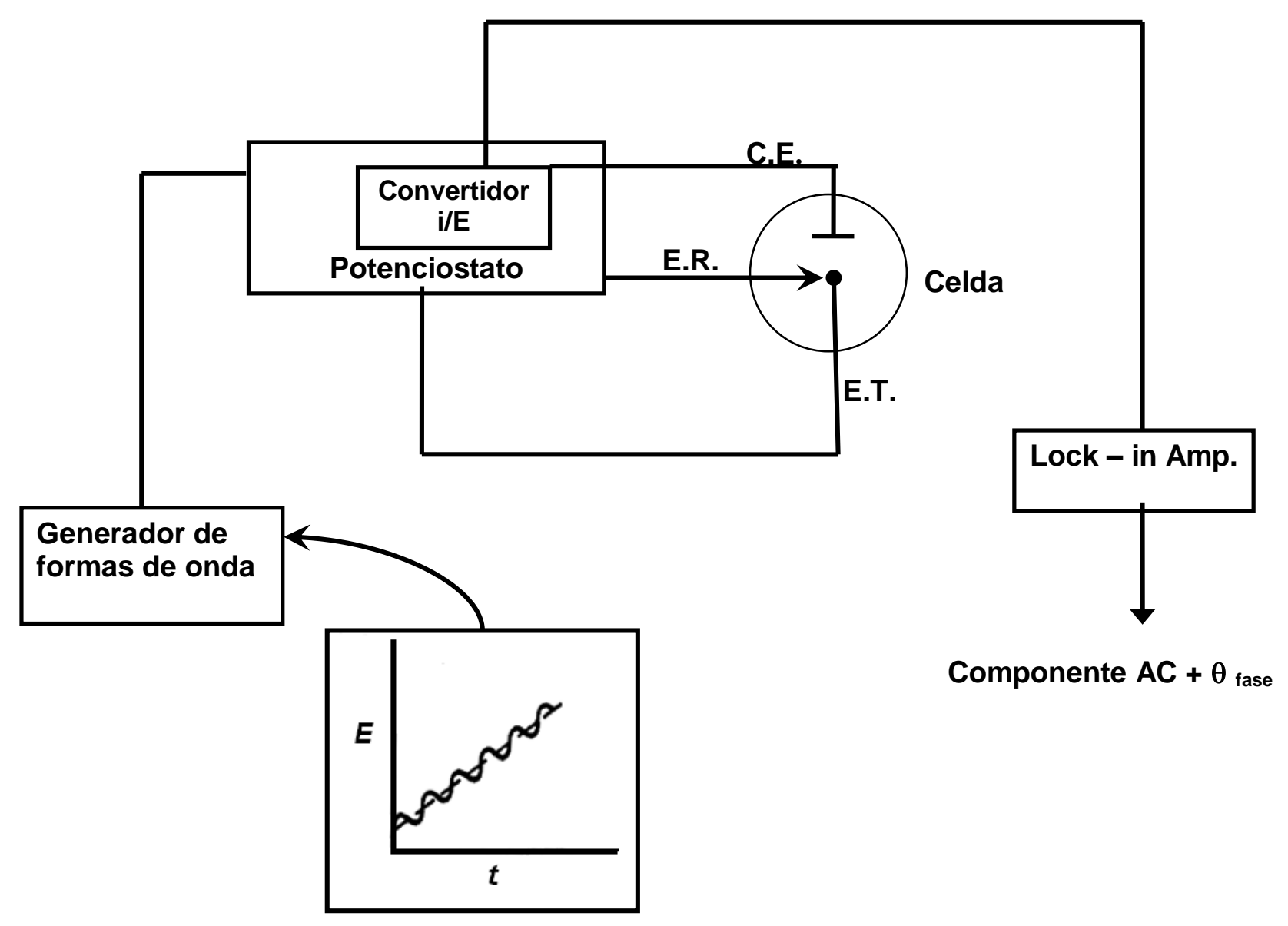

Figura 15. Esquema del equipo utilizado para realizar la voltametría de alterna ACV 


\subsection{Medidas Ópticas In-Situ}

Dentro de los métodos ópticos, convencionalmente se utilizan técnicas espectroscópicas como: UV-Vis, IR y Raman, entre otras. Desde la década de 1960 se ha desarrollado la adaptación de algunas de estas técnicas espectroscópicas para ser aplicadas in situ en una celda electroquímica, a este campo de aplicación se le denomina espectroelectroquímica y constituye una de las áreas activas de la investigación electroquímica (Southampton Electrochemistry Group, 1985).

Las técnicas espectroelectroquímicas son capaces de estudiar la interfase electrodo/solución con el fin de identificar productos e intermediarios de reacción, monitorear la concentración de las diferentes especies presentes y además, determinar propiedades de la superficie misma del electrodo.

Los métodos espectroelectroquímicos son de especial valor en las investigaciones de mecanismos y cinética de procesos electroquímicos, ya que, en principio, permiten la detección y seguimiento de las especies involucradas (o parte de las mismas) a través de su absorción espectral. De esta manera complementan los métodos electroquímicos convencionales para el análisis mecanístico de los procesos debido a que:

(a) Las especies intermediarias se pueden caracterizar mediante las longitudes de onda a las cuales absorben (sujeto a las limitaciones de la superposición de bandas espectrales). 
(b) Permiten detectar cantidades muy pequeñas de intermediarios (cambios de absorbancia de una parte en $\left.10^{5},\left(\Delta \mathrm{R} / \mathrm{R} \sim 1 \times 10^{-5}\right)\right)$.

(c) Las especies involucradas en reacciones químicas que siguen o forman parte de procesos electroquímicos, también pueden ser monitoreadas empleando condiciones de circuito abierto (corriente nula), (Bewick, A. et al.,1980), tal como se utilizó en el análisis del complejo [(bpy) ${ }_{2} \mathrm{Ru}(\operatorname{tatpp}) \mathrm{Cl}_{2}$ $\left(3^{2+}\right)$ en el capítulo 4 (Lezna, R.O., Muñoz Zúñiga, J. et al., 2009), donde se detectó las presencia de una reacción química, mediante la apertura del circuito inmediatamente después de un barrido lineal de potencial sin detener las medidas espectroscópicas.

Las medidas de reflexión en sistemas donde la interfase se puede asimilar a un conjunto de capas ópticamente planas se realizan principalmente con dos configuraciones básicas: reflexión externa e interna. En el caso de la reflexión externa, la luz se propaga en medios ópticos donde en la fase incidente el índice de refracción $\mathbf{n}_{1}$ es menor que el índice de refracción en el medio de transmisión $\mathbf{n}_{\mathbf{2}}\left(\mathrm{n}_{1}<\mathrm{n}_{2}\right)$, mientras que en el caso de la reflexión interna, RI, (generalmente reflexión total atenuada $(A T R))$ el medio de transmisión es ópticamente menos denso que el medio de incidencia $\mathrm{n}_{1}>\mathrm{n}_{2}$ (Steiner, G.,2003, Hecht, E., 1975).

A continuación se describen técnicas espectroelectroquímicas: espectroscopía UV-vis utilizando celdas con electrodos de capa fina ópticamente transparentes - (OTTLEs), reflectancia total atenuada (ATR) y espectroscopía de reflectancia modulada especular externa. La última es utilizada en este trabajo para estudiar los sistemas investigados en el desarrollo de esta tesis. 


\subsubsection{Espectroscopía UV-vis - (OTTLEs)}

El uso de celdas con electrodos de capa fina, ópticamente transparentes (OTTLEs), consiste en una celda de placas paralelas que están separadas por un espaciador de ca. $100 \mu \mathrm{m}$ (utilizado para asegurar el cierre), como se muestra en la figura 16; estas placas contienen un electrodo semitransparente en forma de grilla (una hoja delgada que puede ser de oro con 100 / 2000 perforaciones por pulgada capaz de transmitir entre el $20 \%$ y el $80 \%$ de luz visible) (Southampton Electrochemistry Group,1985). Aunque las grillas son en sí mismas bastante frágiles, una vez montado el sistema resulta robusto (Murray, R.W. et al., 1967).

La intensidad luminosa emergente medida, aparece atenuada por la concentración de las especies absorbentes en el camino óptico, ya sean reactivos, productos y/o intermediarios (Bard, A. J. y Faulkner, L. R. 2001).

El llenado de la celda puede hacerse por acción capilar o mediante la aplicación de vacío en la parte superior. En la figura 16, podemos observar que el recipiente (h) contiene el electrodo de trabajo, el contraelectrodo y el electrodo de referencia. Las características de la celda electrolítica son las de sistemas convencionales de capa fina (Bard, A. J. y Faulkner, L. R. 2001). 


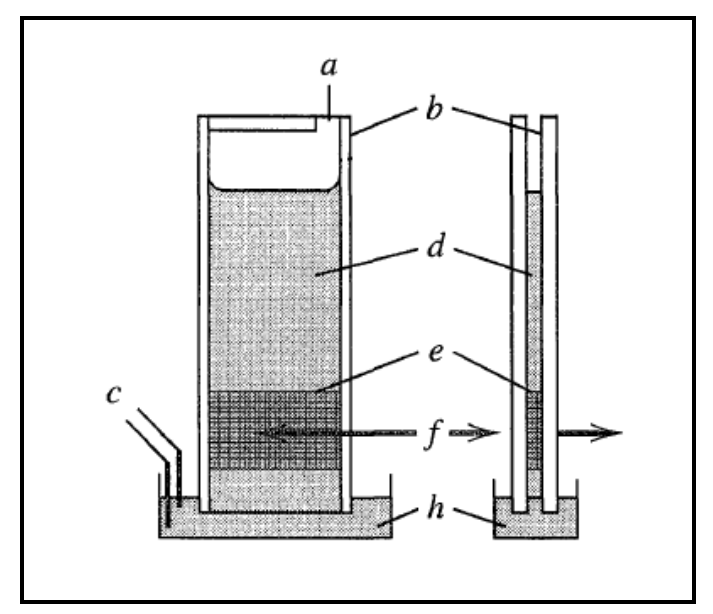

Figura 16. Celda de capa fina con un electrodo de grilla ópticamente transparente; a. Lugar por donde se adiciona la solución, b. Espaciador de teflón, c. Electrodos de referencia y auxiliar, $d$. Solución para la medida, e. Grilla de oro, $1 \mathrm{~cm}$ de alto, f. Dirección del haz óptico

Los materiales para construir OTTLEs comprenden principalmente: grillas de oro, películas de metal evaporado, grillas de níquel y cobre, mercurio electrodepositado en grillas, y carbono vítreo reticulado. Las principales ventajas de los OTTLEs incluyen su construcción en forma rápida y barata, además de su uso directo en un espectrómetro convencional (Southampton Electrochemistry Group, 1985).

\subsubsection{Reflectancia Total Atenuada (ATR)}

Matemáticamente la absorbancia es definida como el logaritmo de la relación entre las intensidades de la luz incidente $I_{O}$ y la de la luz transmitida $I_{T}$ (Ec. 22). 


$$
A=\log \left(\frac{I_{o}}{I_{T}}\right)
$$

La ley de Lambert-Beer (Ec. 23) relaciona la absorbancia de las especies a una determinada longitud de onda con su concentración $C$, su coeficiente de absortividad molar $\varepsilon$, y el camino óptico $l$.

$$
A_{(\lambda)}=\varepsilon_{(\lambda)} C l
$$

El principio de ATR consiste en estudiar la atenuación de la radiación incidente en una interfase entre un material transparente con un índice de refracción $n_{1}$ (es decir, el cristal ATR de medida) y un medio transparente con un índice de refracción $n_{2}$ (la solución), $n_{1}>n_{2}$.

El ángulo a partir del cual no existe refracción y toda la luz es reflejada al medio incidente, es llamado ángulo crítico $\theta_{\mathrm{c}}$. La ley de Snell define a $\theta_{\mathrm{c}}$ representándolo en la ecuación 24: (Fa, K. et al.,2004, Hecht, E., 2003)

$$
\theta_{c}(\lambda)=\operatorname{sen}^{-1}\left(\frac{n_{2}(\lambda)}{n_{1}(\lambda)}\right)
$$

En el caso de un ángulo de incidencia $\theta_{1}$ mayor que $\theta_{c}$, se produce una reflexión interna total. La radiación es completamente reflejada en el cristal en la fase $n_{1}$ ópticamente más densa (Figura 17 a), sin embargo, una onda evanescente penetra en la solución con una profundidad $d_{p}$ del orden de $\lambda / 10$.

Contrario a la espectroscopía de transmisión, en la espectroscopia ATR el camino óptico $l$ no es constante porque la intensidad de la penetración $d_{p}$ de la onda evanescente en la solución es una función del ángulo de incidencia $\theta$, de la 
longitud de onda $\lambda$, y de los índices de refracción $n_{1}$ y $n_{2}$ ( $n_{2}$ varía con la temperatura y longitud de onda) (Billot, P. et al.,2010).

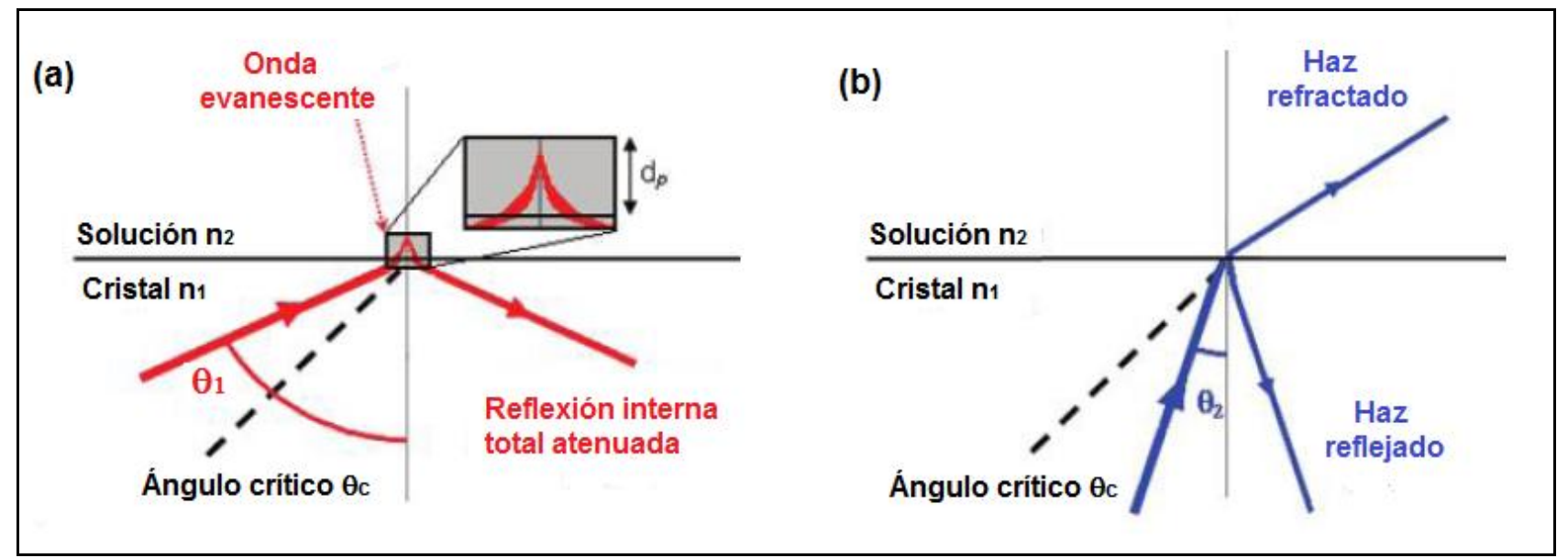

Figura 17. (a) Reflexión interna total. (b) Reflexión externa.

La interacción (absorción) entre la onda evanescente y las especies presentes en solución, resulta en una atenuación del haz reflejado, proporcional a la intensidad de absorción de las especies. Las bandas obtenidas por ATR aparecen distorsionadas respecto a las normales por características de la técnica, la corrección por estas distorsiones se realiza normalmente por computación.

En el caso de un ángulo menor de incidencia $\theta_{2}$ (figura 17 b), la radiación incidente es parcialmente reflejada y refractada. Este fenómeno es llamado reflexión externa.

Para un dispositivo comercial de ATR, el ángulo de incidencia y el índice de refracción del cristal $n_{1}$ son característicos del sistema. Por lo tanto, en lugar de un ángulo crítico, se puede definir un índice de refracción crítico para la solución $n_{\text {crit }}$ (Ec. 25) por encima del cual la reflexión interna total no se observa y domina la reflexión externa. 


$$
n_{\text {crit }}=n_{1} \operatorname{sen} \theta
$$

La atenuación del haz reflejado se mide con un espectrofotómetro UV.

\subsubsection{Espectroscopía de reflectancia modulada especular}

\subsubsection{Reflectancia externa: Figura 18}

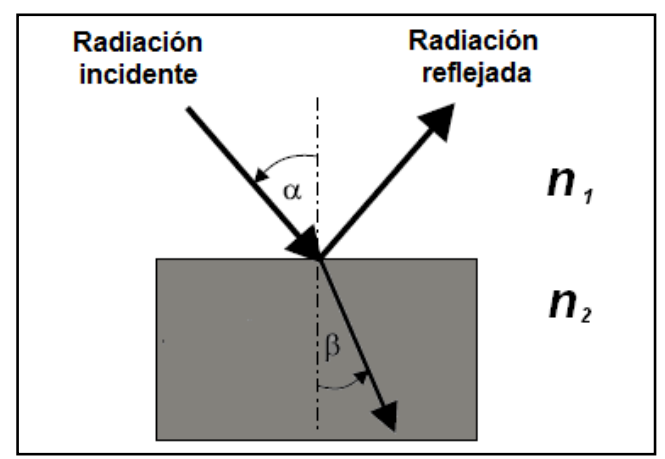

Figura 18. Esquema de la medición de reflectancia externa, $\alpha$ ángulo de incidencia y $\beta$ ángulo de refracción.

La distribución de la intensidad entre la luz transmitida y reflejada en la interfase (aproximada como un plano óptico) se basa en la teoría de Maxwell y en las ecuaciones de Fresnel. La reflectancia $R$ es la relación entre la intensidad $I_{R}$ de la luz reflejada y la intensidad lo de la luz incidente.

$$
R=|r|^{2}=\frac{I_{R}}{I_{O}}
$$

Donde $r$ (número complejo) es el coeficiente de amplitud de reflexión. (Hecht, E. al.,2003) 
La medida de la reflectancia $\mathrm{R}$ depende de la polarización de la radiación, los coeficientes de amplitud para la polarización paralela $r_{\|}$y perpendicular $r_{\perp}$ se encuentran dados en la ecuación 27 y 28 respectivamente:

$$
\begin{array}{r}
r_{\|}=\frac{n_{2} \cos \alpha-n_{1} \cos \beta}{n_{2} \cos \beta+n_{1} \cos \alpha} \\
r_{\perp}=\frac{n_{1} \cos \alpha-n_{2} \cos \beta}{n_{2} \cos \beta+n_{1} \cos \alpha}
\end{array}
$$

Para calcular la intensidad transmitida $T=(1-R)$, se realiza el cálculo de manera similar al de la ecuación 26 donde se tienen en cuenta las intensidades en los diferentes medios (Ec. 29).

$$
I_{T}=(1-R) I_{O}
$$

El índice de refracción se define como:

$$
n=\frac{c}{v_{p}}
$$

Donde $c$ es la velocidad de la luz en el vacío y $v_{p}$ es la velocidad de fase de la luz en el medio en estudio (la velocidad de transmisión de energía está dada por la velocidad de grupo, $\mathrm{v}_{\mathrm{g}}$ ).

Los índices $\mathrm{n}_{\mathrm{i}}$ son números reales (Ec. 30), que pueden ser reemplazados por su forma compleja $(\hat{n})$ cuando el medio en estudio absorbe (disipa) radiación, de esta manera se conserva la forma y la validez de las ecuaciones de Fresnel (Ec. 31):

$$
\hat{n}=n-i k
$$


Donde $k$ es el índice de absorción, que se relaciona con el coeficiente de absortividad molar $\varepsilon$, normalmente empleado en la ley de Lambert-Beer (Ec. 32) (Steiner, G.,2003) de acuerdo a:

$$
k=\frac{\varepsilon C \lambda \ln 10}{4 \pi}
$$

Donde $C$ es la concentración.

\section{Reflectancia especular}

Es una técnica de reflexión externa donde el rayo de luz incide desde la solución y es reflejado en el electrodo (Kuwana,T.,1973). El haz incidente, generalmente monocromático (en el OMA es policromático), está usualmente polarizado en un plano paralelo $(\| \circ \mathrm{p})$ o perpendicular $(\perp \circ \mathrm{s})$ al plano de incidencia; se registra la intensidad del rayo reflejado en función de la longitud de onda $\mathrm{y} / \mathrm{o}$ del potencial $\mathrm{y} / \mathrm{o}$ del tiempo (Southampton Electrochemistry Group, 1985).

\subsubsection{Espectroscopía de reflectancia para especies adsorbidas sobre} la superficie del electrodo

La teoría general de la reflectancia de películas adsorbidas en la interfase, modelada o aproximada como sistema de tres fases, permite que las expresiones para $\Delta R / R$ en sistemas multifásicos se puedan reducir a una forma más sencilla en el caso de películas delgadas (respecto a $\lambda$ ) vide infra Ec. 33 (Mclntyre, J.D.E. et al., 1971).

Utilizando espectroscopía de reflectancia se realizan, entre otras investigaciones, estudios de propiedades ópticas de sólidos, propiedades de 
películas delgadas, productos e intermediarios de una reacción (química y/o electroquímica), etc. Si medimos $\Delta R / R$ durante el curso real de un experimento, se puede determinar el cubrimiento de la superficie por una especie adsorbida, incluso en presencia de otra reacción en la superficie. La técnica es de importancia también para los estudios de mecanismos en catálisis heterogénea y de electroreflectancia de semiconductores.

Consideremos las propiedades ópticas de películas delgadas cuyo espesor, $d$, sea mucho menor que la longitud de onda, $\lambda$, de la radiación incidente sobre la superficie. Se ha demostrado que mediante una aproximación lineal válida para $\mathrm{d}<<\lambda$ (c.a 1/1000), las expresiones complejas de reflexión para sistemas multifásicos, ecuaciones de Fresnel, (Figura 19) se pueden reducir a una forma más simple que aporta una idea física más directa de las propiedades de estos sistemas. El cambio de reflectancia producido por la formación de una película delgada sobre la superficie del sustrato (electrodo) está relacionado con el espesor de la película, la cobertura de la superficie, la longitud de onda, la constante dieléctrica de la solución, del sustrato y de la película misma (Mclntyre, J.D.E. et al., 1971).

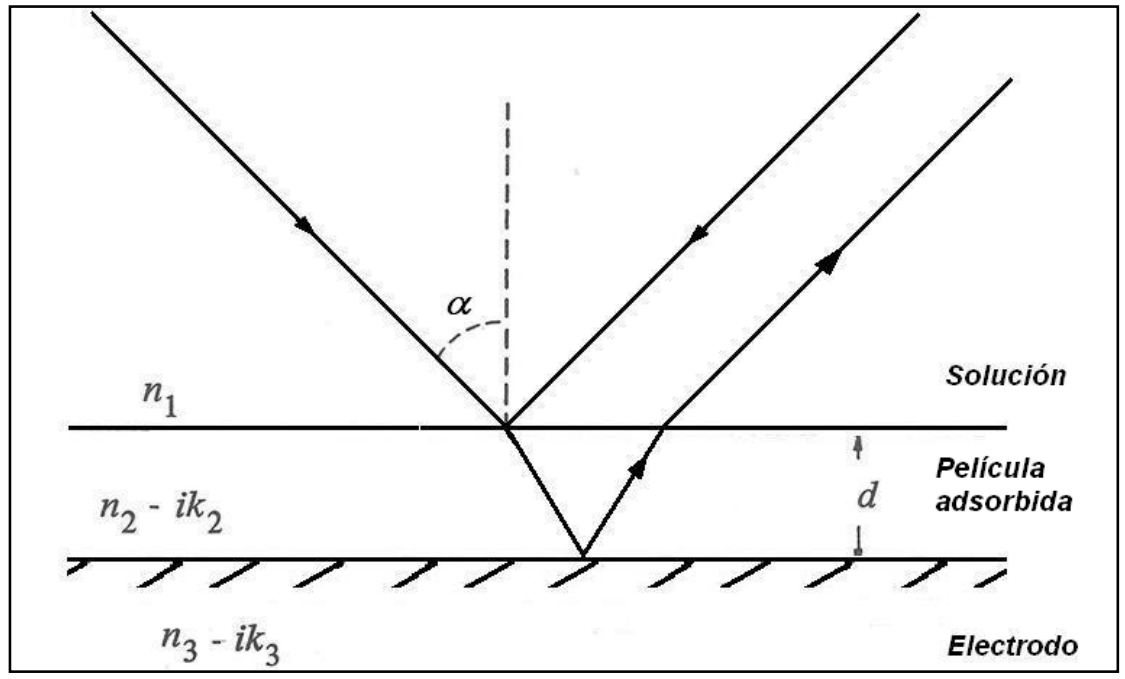

Figura 19. Modelo de tres capas ópticas para la interfase electrodo-solución. 
En este caso, cuando $d<<\lambda$, pueden hacerse ciertas aproximaciones, que simplifiquen el análisis del cambio de reflectividad y que conducen a la forma:

$$
\begin{aligned}
& \left(\frac{\Delta R}{R}\right)_{\perp}=\frac{8 \pi d n_{1} \cos \alpha}{\lambda} \operatorname{Im}\left(\frac{\left(\hat{\epsilon}_{2}-\hat{\epsilon}_{3}\right)}{\left(\epsilon_{1}-\hat{\epsilon}_{3}\right)}\right) \\
& \left(\frac{\Delta R}{R}\right)_{\|}=\frac{8 \pi d n_{1} \cos \alpha}{\lambda} \operatorname{Im}\left(\frac{\left(\hat{\epsilon}_{2}-\hat{\epsilon}_{3}\right)}{\left(\frac{\epsilon_{1}}{\hat{\epsilon}_{1}-\hat{\epsilon}_{3}}\right)} \frac{1-\left(\hat{\epsilon}_{3}+\hat{\epsilon}_{3}\right) \operatorname{sen}^{2} \alpha}{1-\left(\frac{1}{\hat{\epsilon}_{3}}\right)\left(\epsilon_{1}+\hat{\epsilon}_{3}\right) \operatorname{sen}^{2} \alpha}\right)
\end{aligned}
$$

Donde $\hat{\epsilon}_{n}$ corresponde a la constante dieléctrica compleja de la fase $n, n_{1}$ es el índice de refracción de la fase incidente transparente, e $\operatorname{Im}(x)$ se refiere a la parte imaginaria de la variable $(\mathrm{x})$.

$\hat{\epsilon}_{n}$ se define por la ecuación 35,

$$
\hat{\epsilon}=\epsilon^{\prime}-i \epsilon "
$$

$\mathrm{Y}$, a su vez, $\in^{\prime} \mathrm{y} \in$ "se relacionan con $n$ y $k$ de acuerdo a

$$
\begin{aligned}
& \epsilon^{\prime}=\frac{n^{2}-k^{2}}{\mu} \\
& \epsilon^{\prime \prime}=\frac{2 n k}{\mu}
\end{aligned}
$$

donde $\mu$ es la permeabilidad magnética (Southampton Electrochemistry Group, 1985). 
En nuestro trabajo los sistemas estudiados son especies en solución, supuestamente no adsorbidas, y por consiguiente no se han empleado las ecuaciones de Fresnel.

\subsubsection{Espectroscopía de reflectancia de especies en solución:}

Una de las ventajas de la espectroscopía de reflectancia con modulación de potencial es que se adapta a los estudios tanto de especies en solución como de procesos en la superficie del electrodo, característica no compartida por otras técnicas ópticas.

Los datos obtenidos para especies en solución son analizados de manera similar a la utilizada para investigaciones usando OTE (electrodos ópticamente transparentes) en la cual la absorbancia $A(t, \lambda)$, está dada por la integración de los perfiles de concentración $[C(x, t)]$, ecuación 38 ,

$$
A(t, \lambda)=\varepsilon(\lambda) \int_{0}^{\infty}(C(x, t)) d x
$$

donde $\varepsilon$ es la absortividad molar a una determinada $\lambda$ definido en la ecuación 32 que representa la ecuación de Lambert-Beer para el caso donde $C(x, t)$ es función del tiempo y de la distancia al electrodo formalmente similar al caso de pulsos de potencial.

Para realizar los cálculos de absorbancia total para especies en solución, el valor dado en la ecuación 38 debe ser incrementada por el factor $2 / \cos \alpha$ (que tiene en cuenta el camino óptico de la radiación en el interior de la celda), donde $\alpha$ 
es el ángulo de incidencia usualmente elegido como $45^{\circ}$ o $60^{\circ}$ fijado por el diseño de la celda óptica.

$45^{\circ}$ es el ángulo de Brewster de la interfase (Mclntyre, J.D.E. et al.,1971), es decir aquel que presenta la contribución mínima de la doble capa al cambio de reflectividad. Una característica adicional útil en estudios de reflectancia es que los datos pueden ser obtenidos con polarización de luz $s$ ó p ( $p$ es más intensa en la interfase). Sin embargo para el estudio de especies libres en solución no hay dependencia con el estado de polarización de la luz, mientras que si se estudian procesos en la superficie tales como adsorción los valores de $\Delta R / R$ o $\delta R / R$, dependen del estado de polarización de la radiación (Southampton Electrochemistry Group,1985). Estas propiedades permiten, en principio, diferenciar respuestas de especies en solución de aquellas sobre la superficie.

\subsubsection{Medidas diferenciales}

Las mediciones ópticas diferenciales se realizaron con un espectrómetro computarizado montado en el laboratorio especialmente para estudios ópticos insitu de sistemas electroquímicos. Una lámpara de cuarzo-halógeno de $250 \mathrm{~W}$ es alimentada por una fuente de tensión continua altamente regulada, acoplada a un monocromador doble (para reducir al mínimo la luz parásita/dispersada). La radiación monocromática es llevada a la celda a través de una ventana de cuarzo con un ángulo de incidencia de $45060^{\circ}$. Todas las lentes y las ventanas de las

celdas ópticas están hechas de cuarzo (Spectrosil). Órdenes de difracción superiores son suprimidos por medio de filtros adecuados (Lezna, R.O. et al., 1988 a). 
Las principales contribuciones de la espectroscopía de reflectancia electroquímica han sido el aporte a la dilucidación de mecanismos de reacción, estudios de doble capa, investigaciones de deposición a subpotenciales (UPD), y la investigación del efecto de electroreflectancia (contribución a la reflectancia de los electrones del sustrato (electrodo), ER) en metales y semiconductores (Southampton Electrochemistry Group 1985, Cardona M., 1969).

La figura 20 describe esquemáticamente el equipo de reflectancia con modulación de potencial utilizado tanto para medidas ópticas integrales como diferenciales en la elaboración de esta tesis. La luz monocromática seleccionada mediante un monocromador doble es reflejada por el electrodo de trabajo (en nuestro caso, principalmente, $\mathrm{Pt}, \mathrm{Au}$, etc). Al mismo tiempo el potencial del electrodo es modulado con una señal sinusoidal de ca. $50 \mathrm{mV}$ pico a pico que a su vez modula la intensidad de la luz sobre el electrodo. La radiación reflejada es luego conducida a un fototubo, con sensibilidad hasta el rojo del espectro, que la convierte en una señal eléctrica amplificada de alterna (a.c), esta señal va a un lock-in amplifier que la rectifica a una señal de continua. El tamaño significativo de la amplitud de la modulación, $50 \mathrm{mV}_{\mathrm{pk}-\mathrm{pk}}$, está impuesto por la necesidad de obtener una respuesta óptica (siempre muy pequeña, $10^{-4}-10^{-5}$ ) medible frente al nivel de ruido de fondo.

Los espectros diferenciales permiten ver especies de vida corta, empleando típicamente frecuencias entre $6 \mathrm{~Hz}$ y $33 \mathrm{~Hz}$ trabajando a longitudes de onda fijas. Por ejemplo, en la figura 21, se muestra un espectro para el complejo dinuclear $\left[(\text { phen })_{2} \operatorname{Ru}(\text { tatppa }) \operatorname{Ru}(\text { phen })_{2}\right]^{4+}$ denominado $\mathbf{1}^{+4}$, medido experimentalmente en nuestro laboratorio que será analizado posteriormente en el capítulo 3. 


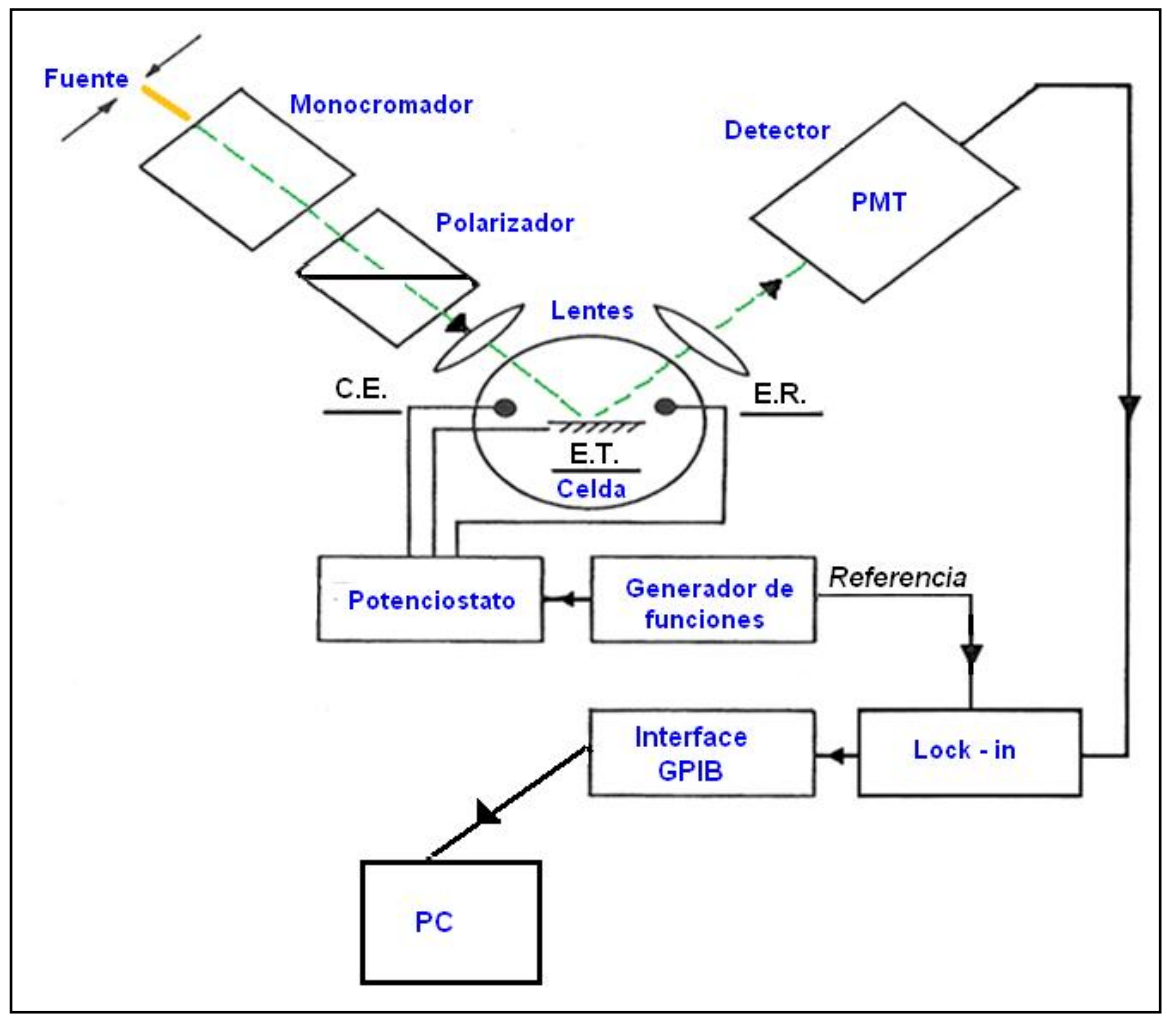

Figura 20. Esquema del equipamiento utilizado en las medidas de reflectancia diferencial.

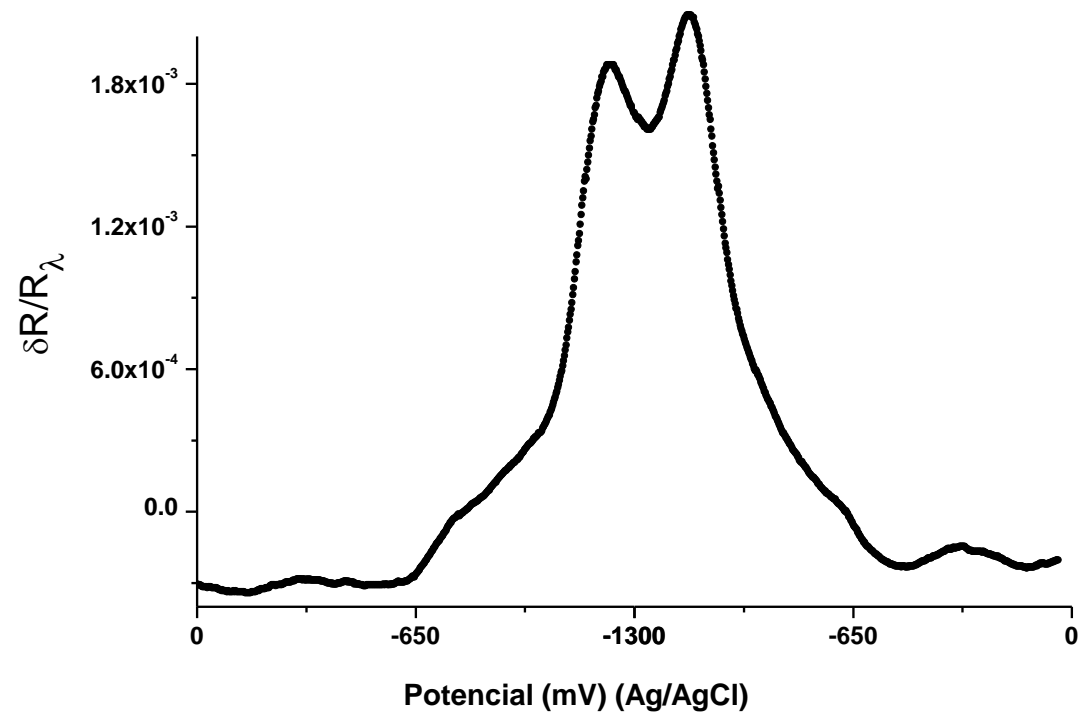

Figura 21. Espectro diferencial para $1^{+4}, \lambda=650 \mathrm{~nm}$, frecuencia $6 \mathrm{~Hz}$. 


\subsubsection{Medidas integrales}

En la figura 22 a, se muestra un ejemplo de un espectro integral para $1^{+4}$, esta medida fue realizada tomando espectros cada $20 \mathrm{mV}$ en estado estacionario utilizando el OMA (descripto en la figura 23) en el intervalo entre $0 \leftrightarrow-1200 \mathrm{mV}$ $(\mathrm{Ag} / \mathrm{AgCl})$.

La respuesta observada en la figura 22 a., se obtuvo a partir de una medida espectroelectroquímica, donde cada uno de los espectros presentes se midieron a un potencial estacionario. A medida que aumenta el potencial (negativamente), se observa el crecimiento de una banda cuyo signo $(\downarrow)$ indica aparición de nuevas especies producto de la reacción de reducción, en este caso la banda principal se detecta aproximadamente a $500 \mathrm{~nm}$. Es decir, se observa un aumento en la intensidad de una banda proporcional a la concentración de una nueva especie generada. Este aumento es medido de acuerdo a la relación descripta por la ecuación 39,

$$
\frac{\Delta R}{R}=\frac{\left(R_{i}-R_{O}\right)}{R_{O}}
$$

donde, $\mathrm{R}_{0}$ corresponde al espectro de referencia (a un potencial donde no ha comenzado la reducción). $R_{i}$ corresponde a los espectros medidos a los diferentes potenciales estacionarios.

En la figura 22 b., se muestra, en negro, la intensidad de la respuesta generada a longitudes de onda seleccionadas, medida como la altura de la banda, 
en función de las especies que se investigan (en este caso $505 \mathrm{~nm}$ ). Este gráfico (figura 22 b) es útil para medir la variación de las concentraciones de las especies formadas en función del potencial o, eventualmente, en función del tiempo (en caso de apertura de circuito).

Para realizar las medidas mostradas en la figura 22 se utiliza un OMA (optical multichannel analizar) figura 23 , que consiste en una lámpara de cuarzohalógeno que ilumina con luz blanca el electrodo de trabajo pulido a espejo (por ejemplo $\mathrm{Pt}$, Au policristalinos), la luz reflejada es llevada al policromador que dispersa la luz en sus colores desde el UV hasta el IR cercano sobre una matriz de diodos que cuantifica la intensidad de cada $\lambda$.

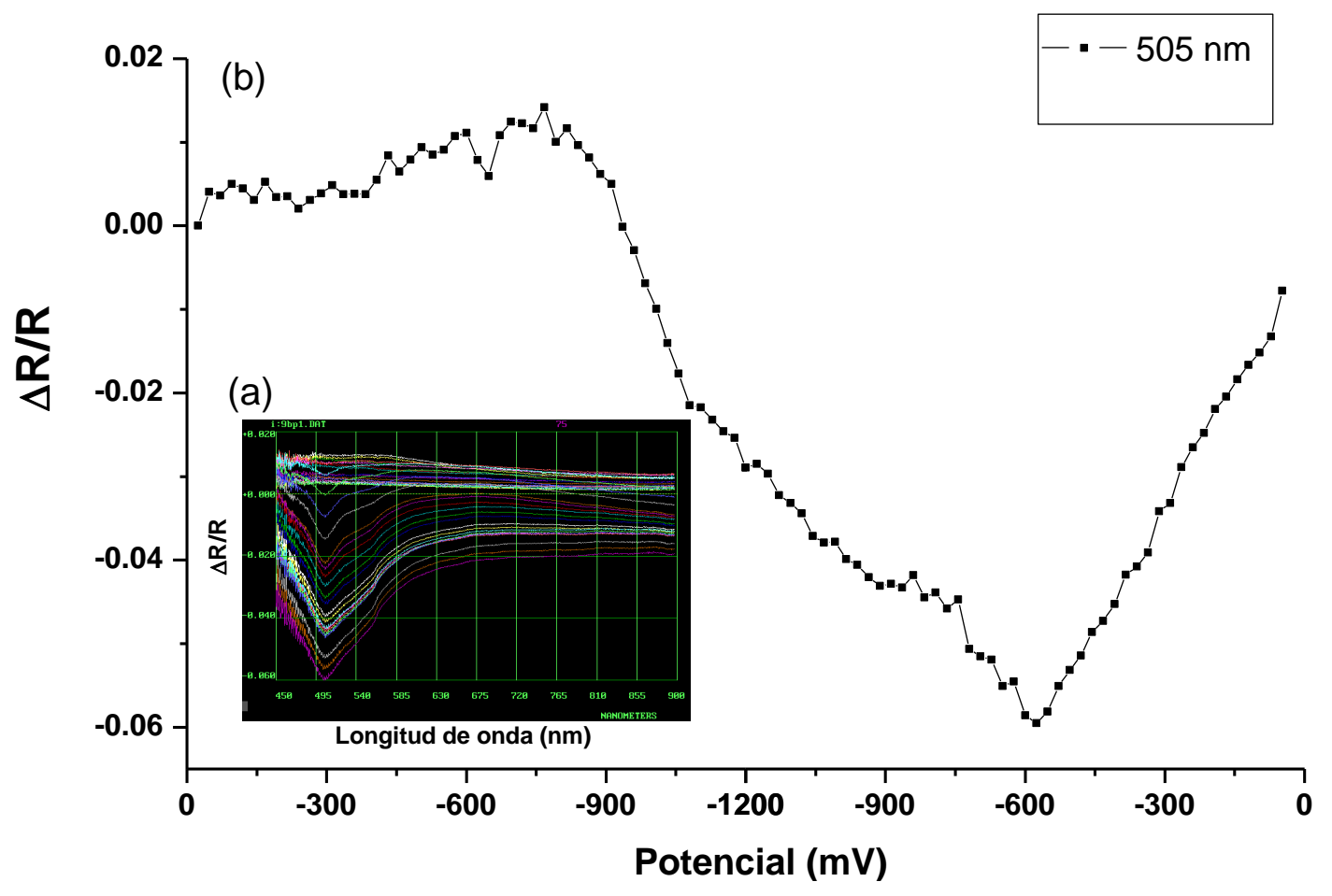

Figura 22. (a) Espectro integral para $1^{+4}$, cada $20 m V, 0 \leftrightarrow-1200 m V$.

(b) Relaciones intensidad vs potencial para $505 \mathrm{~nm}$. 


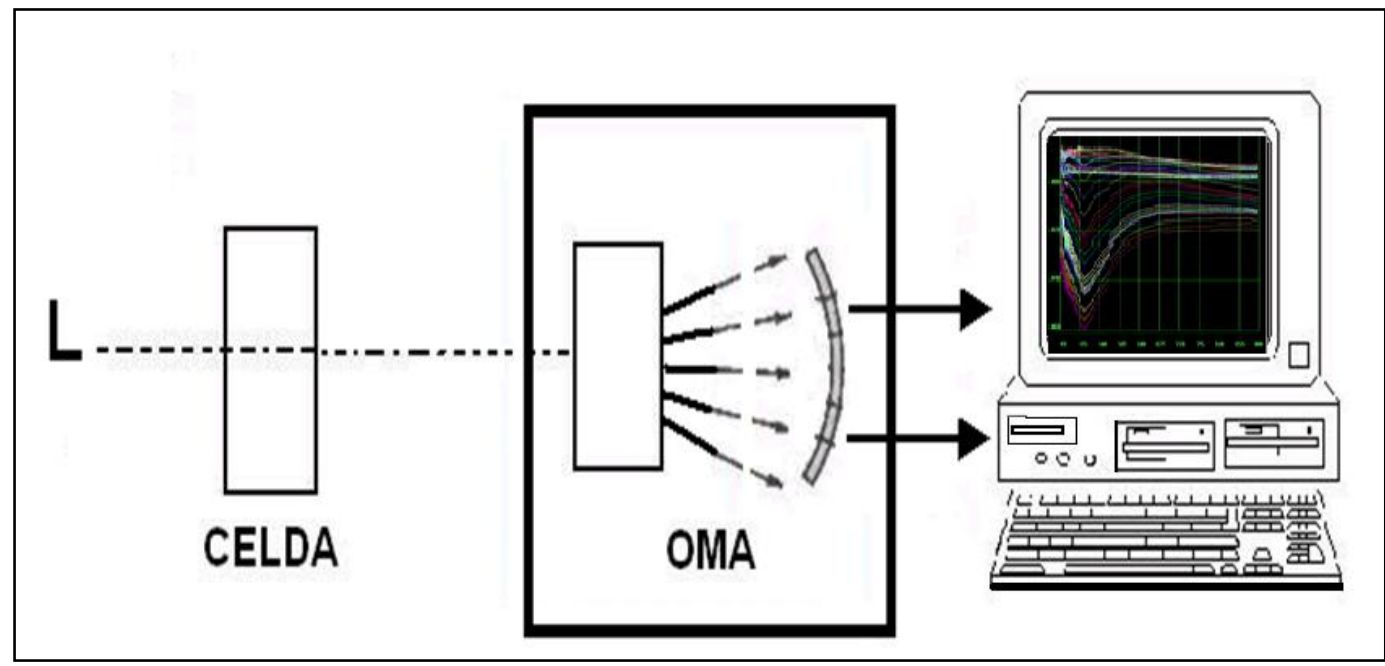

Figura 23. Esquema representativo del equipamiento (utilizando OMA) para realizar medidas integrales. 


\subsection{Referencias}

Bard, A. J. y Faulkner, L. R; ELECTROCHEMICAL METHODS FUNDAMENTALS AND APPLICATIONS. 2001, 2da. Ed. Wiley \& Sons. Cap 6.

Bewick, A.; Mellor, J. M., Pons, B. S. Electrochim. Acta, 1980, 25 (7), 931 941.

Billot, P.; Couty, M. y Hosek, P. Organic Process Research \& Development, 2010, 14, 511 - 523.

Bockris, J.O'M. y Reddy, A.K.N.; ELECTROQUÍMICA MODERNA. 2006, Vol 2. Reverté. España. 665-671.

Bracco, L.L.B.; Lezna, R. O.; Muñoz Zuñiga, J.; Ruiz, G.T.; Féliz, M.R.; Ferraudi, G.J.; Einschlag García, F. S.; Wolcan, E. Inorganica Chimica Acta. 2011, 370, 482-491.

Cardona, M. MODULATION SPECTROSCOPY . 1969. Academic Press.

Fa, K.; Jiang, T.; Nalaskowski, J. y Miller, J.D. Langmuir, 2004, 20, 53115321.

Harris, D.C. Análisis Químico Cuántitativo. 1992, Iberoamericana S.A. Mexico D.F. $451-475$. 
Hecht, E.; Schaum's Outline Of Theory And Problems Of Optics. 1975, Ed. McGraw-Hill. 33-47.

Hecht, E. Optics. 2003, 4ta edición, Ed. Adisson - Wesley.

Juliarena, M. P.; Lezna, R. O.; Feliz, M. R.; Ruiz, G. T.; Thomas, S.; Ferraudi, G. y Carmichael I. J. Org. Chem. 2006, 71, 2870-2873.

Kuwana, T., Berichte der Bunsengesellschaft für Physikalische Chemie, 1973, $77,858-871$.

Lezna, R.O.; de Tacconi, N.R.; Hahn F. y Arvia, A.J. J. Electroanal. Chem. 1986, 306, 259-269.

Lezna, R.O.; de Tacconi, N.R. y Arvia, A.J. J. Electroanal. Chem. 1988a, 255, 251-266.

Lezna, R.O. ; de Tacconi, N.R. ; Rapallini, J.A. y Arvia, A.J. Anal. Asoc. Quím. Argent., 1988b, 76(1), 25.

Lezna, R.O. ; Rajeshwar, K. y de Tacconi, N.R. Analytical Chemistry, 1992, 64 (7), 429A.

Lezna, R.O., Anal. Asoc. Quím. Argent., 1994, 82(4), 293-304.

Lezna, R. O.; de Tacconi, N. R.; Janaratne, T.; Muñoz Zúñiga, J. y MacDonnell F. M. J. Argent. Chem. Soc., 2009, 97(1), 273-288.

Mcintyre, J.D.E. y Aspnes, D. E. Surface Science, 1971, 24, 417-434

Murray, R.W.; Heineman, W. R. y O'Dom, G. W. Anaytical Chemistry, 1967, 39, 1666-1668. 
Ruiz, G. T.; Juliarena, M. P.; Lezna, R. O.; Feliz, M. R.; Ferraudi, G. Journal of Photochemistry and Photobiology A: Chemistry. 2006, 179, $289-297$.

Shreeyukta, S.; de Tacconi, N.R.; Diaz, N.R.G.; Lezna, R. O.; Muñoz Zúñiga, J.; Abayan, K. y MacDonnell, F.M. Inorg. Chem. 2011, 50 (19), 9318-9328.

Southampton Electrochemistry Group. Instrumental Methods In Electrochemistry, Ellis Horwood Limited 1985, Cap. 10.

Steiner, G. Handbook of Spectroscopy. Editado por Günter Gauglitz y Tuan Vo-Dinh, 2003, (1) 70-87.

Tacconi, N.R.; Rajeshwar, K. y Lezna, R.O. Chem. Mat, 2003, 15(16) 3046 3062; (Review)

Tacconi, N. R.; Lezna, R. O.; Konduri, R.; Ongeri, F.; Rajeshwar, K. y MacDonnell, F. M. Chem. Eur. J. 2005, 11, 4327-4339.

Tacconi, N. R.; Lezna, R. O.; Chitakunye, R. y MacDonnell, F.M. Inorg. Chem. 2008, 47,8847-8858.

Wouters, K.L.; de Tacconi, N.R.; Konduri,R.; Lezna, R.O. y MacDonnell, F.M. Photosynthesis Research, 2006, 87, 41-55.

William T. Y. Journal of Electroanalytical Chemistry, 1998, 454, 33-38. 


\section{Electroquímica y}

espectroelectroquímica del complejo

$\left[(\text { phen })_{2} \operatorname{Ru}(\text { tatpp } \alpha) \operatorname{Ru}(\text { phen })_{2}\right]^{4+}$

$\left(1^{4+}\right)$ 
Electroquímica y Espectroelectroquímica de $1^{4+} \mid 2$

Capítulo 3. 
Capítulo 3. Electroquímica y espectroelectroquímica del complejo $\left[(\text { phen })_{2} \operatorname{Ru}(\text { tatpp } \alpha) \operatorname{Ru}(\text { phen })_{2}\right]^{4+}\left(1^{4+}\right)$

3.1 Introducción

3.2 Electroquímica de $\left[(\text { phen })_{2} R u(\text { tatpp } \alpha) R u(\text { phen })_{2}\right]^{4+}\left(1^{4+}\right)$

3.3 Espectro electrónico de $\mathbf{1}^{4+}$

3.4 Características de la forma oxidada y reducida de $\mathbf{1}^{4+}$

3.5 Espectroelectroquímica del complejo $\left[(\text { phen })_{2} R u(\text { tatpp } \alpha) R u(\text { phen })_{2}\right]^{4+}$ $\left(1^{4+}\right)$

3.6 Discusión

3.7 Conclusiones.

3.8 Referencias. 


\subsection{Introducción}

Los complejos de rutenio polipiridilo pueden actuar potencialmente como cromóforos en procesos similares a una fotosíntesis artificial, con el objetivo de producir combustibles útiles a partir de agua, $\mathrm{CO}_{2}$, etc., con la ayuda de energía solar. (Meyer, T., 1989, Amouyal, E. et al., 1995, Campagna, S. et al., 2007). Las reacciones de desprendimiento de hidrógeno y oxígeno están entre las más promisorias de los esquemas de generación práctica de combustibles. (MacDonnell, F. M., 2008). Las reacciones de formación de combustibles de interés son procesos multielectrónicos, sin embargo la mayoría de los fotocatalizadores desarrollados hasta el presente son sólo capaces de excitar y almacenar un electrón por fotoexcitación (Watts, R. J. et al., 1991). Si bien es posible conducir estas reacciones multielectrónicas en pasos de un electrón (Barton, C. E. et al., 2010), se puede mejorar la eficiencia de las mismas con el uso de catalizadores capaces de acelerar reducciones multielectrónicas (Watts, $R$. J. et al., 1991).

Los grupos de Brewer, (Elvington, M. et al., 2006, 2007, Holder, A. A. et al., 2004), Nocera, (Heyduk, A. F. et al., 2001, Esswein, A. J. et al., 2005, Rosenthal, J. et al., 2005) y Bocarsly (Pfennig, B. W. et al., 1999, 2002) han investigado complejos fotoquímicamente activos algunos de los cuales catalizan la formación de $\mathrm{H}_{2}$ en condiciones adecuadas. De éstos, sólo un complejo, el trímero $\mathrm{Ru}^{\mathrm{II}}$ - Ir"II$\mathrm{Ru}$ ", es capaz de almacenar electrones en los orbitales del ligando aceptor, en este caso, los dos electrones son almacenados en diferentes ligandos (Molnar, $S$. M. et al., 1994). 
Existen varias publicaciones donde se utilizan complejos de rutenio polipiridilo como cromóforos para la formación fotocatalítica de $\mathrm{H}_{2}$, (Hawecker, J. et al., 1983, Rau, S. et al., 2006, Ozawa, H. et al., 2006, Masayuki, K. et al., 2010, Amouyal, E. et al., 1980, Kirch, M. et al., 1979), normalmente adicionando un co-catalizador a la solución o combinándolo directamente al cromóforo de rutenio, sin embargo, el mecanismo y los intermediarios a través de los cuales se forma $\mathrm{H}_{2}$ no están bien definidos.

En nuestro grupo se analizaron diferentes complejos de $\mathrm{Ru}$ con el fin de estudiar su actividad fotocatalítica (Tacconi, N.R., Lezna, R.O. et al., 2005, Wouters, K.L. et al., 2006, Tacconi, N. R., Lezna, R.O. et al., 2008a;2008b, Lezna, R.O., Muñoz Zúñiga, J. et al., 2009, Shreeyukta, S. et al., 2011). Uno de los trabajos publicados es la síntesis y caracterización del complejo dinuclear $\left[\left(\text { phen) }{ }_{2} \mathrm{Ru}(\operatorname{tatpp} \beta) \mathrm{Ru}(\text { phen })_{2}\right]^{4+}\right.$ denominado $2^{4+}$ (figura 1). (Kim, M-J. et al., 2002, Konduri, R. et al., 2004, Tacconi, N. R., Lezna, R.O. et al., 2005). $\mathbf{2}^{4+}$ es un complejo con una longitud mayor de $2 \mathrm{~nm}$ que posee una carga de +4 de los dos iones $\mathrm{Ru}$ (II) coordinados al ligando puente $[9,11,20,22$ - tetraazatetrapirido [3,2a:2'3'- c:3",2"- I:2"',3'] pentaceno] denominado tatpp $\beta$ (Konduri, R. et al., 2004); este ligando puente central juega un papel en las propiedades fotofísicas y redox del complejo (Kim, M-J. et al., 2002) ya que es el lugar destinado al almacenamiento electrónico, vide infra. $2^{4+}$ posee propiedades adecuadas para los objetivos buscados, tales como fuerte absorción en la región del espectro solar, capacidad de almacenamiento de dos electrones y fotoestabilidad ante la luz visible, (Kim, M-J. et al., 2002, Konduri, R. et al., 2004, Tacconi, N. R., Lezna, R.O. et al., 2005) sin embargo, sus potenciales de reducción no son lo suficientemente negativos para producir hidrógeno de manera eficiente, es decir, la fuerza impulsora para la transferencia de los electrones almacenados no es significativa. 


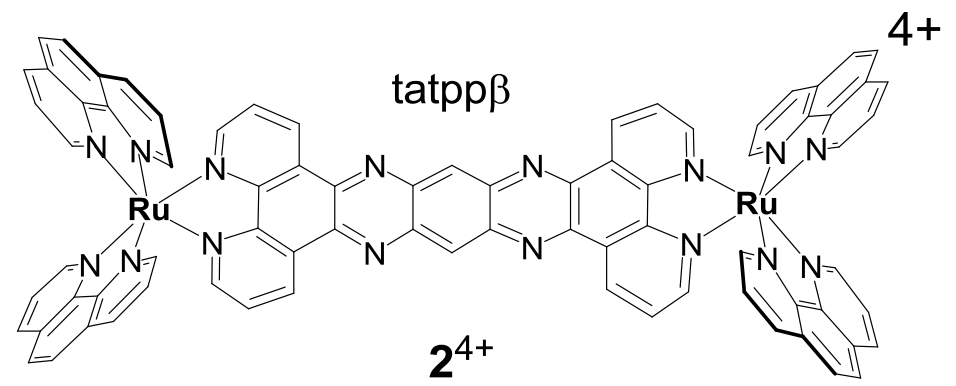

Figura 1. Estructura del complejo $\left[(\text { phen })_{2} R u(\operatorname{tatpp} \beta) R u(\text { phen })_{2}\right]^{4+}$, denominado $2^{4+}$

Estos resultados sugieren encaminar la búsqueda hacia complejos con mayor capacidad de almacenamiento electrónico y con potenciales de reducción más negativos. A tal efecto se ha sintetizado el complejo $\left[(\text { phen })_{2} R u(\text { tatpp } \alpha) R u(\text { phen })_{2}\right]^{4+}$ que denominaremos $1^{4+}$ (Shreeyukta, S., Muñoz Zúñiga, J. et al., 2011). $\mathbf{1}^{4+}$ es un isómero geométrico de $\mathbf{2}^{4+}$, ambos complejos contienen unidades fenantrolinas como ligandos axiales y se diferencian en el ligando puente central: tatpp $\alpha$ para $\mathbf{1}^{+4}$ y tatpp $\beta$ para $\mathbf{2}^{4+}$ (figura 2).

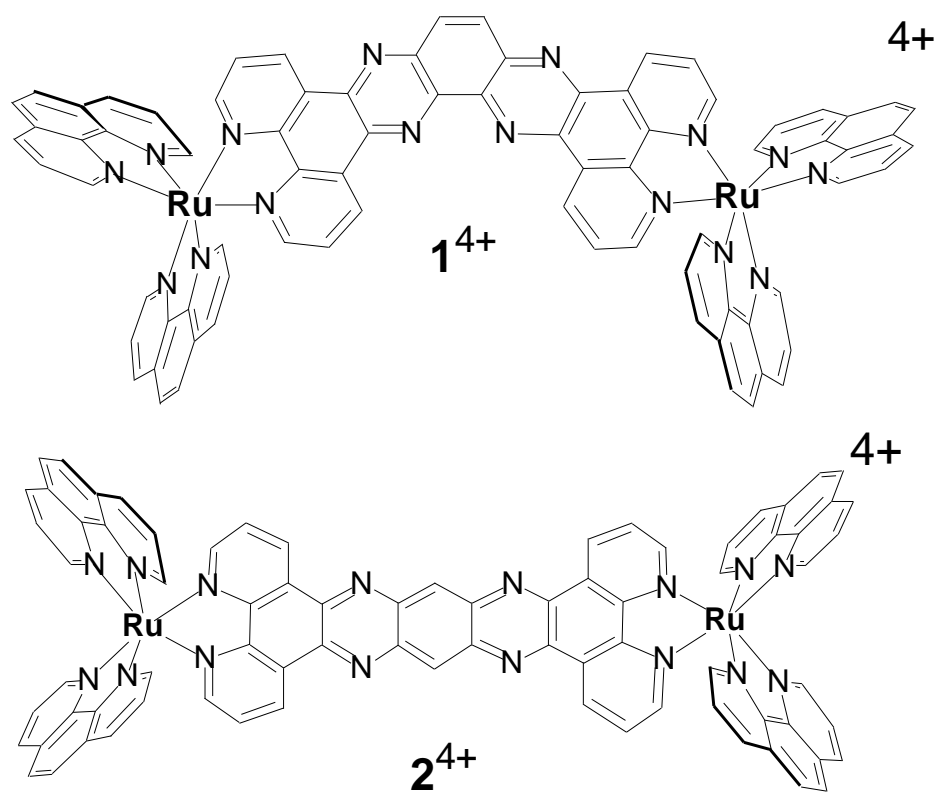

Figura 2. Estructura para los complejos $\mathbf{1}^{+4}$ y $\mathbf{2}^{+4}$ 
El ligando tatpp $\alpha$ tiene una estructura similar, con la misma fórmula química que su análogo tatpp $\beta$. La diferencia radica en que el ligando tatpp $\beta$ es lineal, mientras que tatpp $\alpha$ tiene una sustitución orto $(\alpha)$ sobre el benceno central, conduciendo a la aparición de un ángulo de $120^{\circ}$ en la estructura.

Mediante cálculos de orbitales moleculares (OM) utilizando el método DFT, (Density Functional Theory) (Ghosh, S.K. et al., 1982; Nagy, Á. 1998), se ha demostrado que el LUMO de $1^{+4}$ tiene una energía mayor que la correspondiente al complejo $\mathbf{2}^{+4}$, es decir, $\mathbf{1}^{+4}$ debería poseer un mayor potencial de reducción que su análogo lineal (Shreeyukta, S. et al., 2011). Teniendo en cuenta este resultado se realizaron medidas electroquímicas y espectroelectroquímicas para verificar la viabilidad de $\mathbf{1}^{+4}$ como fotocatalizador. 


\subsection{Electroquímica de $\left[(\text { phen })_{2} R u(\text { tatpp } \alpha) R u(\text { phen })_{2}\right]^{4+}$}

Se utilizaron técnicas de voltametría cíclica $(\mathrm{CV})$ y de alterna (ACV) para realizar el estudio electroquímico al complejo $\left[(\text { phen })_{2} R u(\operatorname{tatpp} \alpha) \operatorname{Ru}(\text { phen })_{2}\right]^{4+}\left(\mathbf{1}^{+4}\right)$.

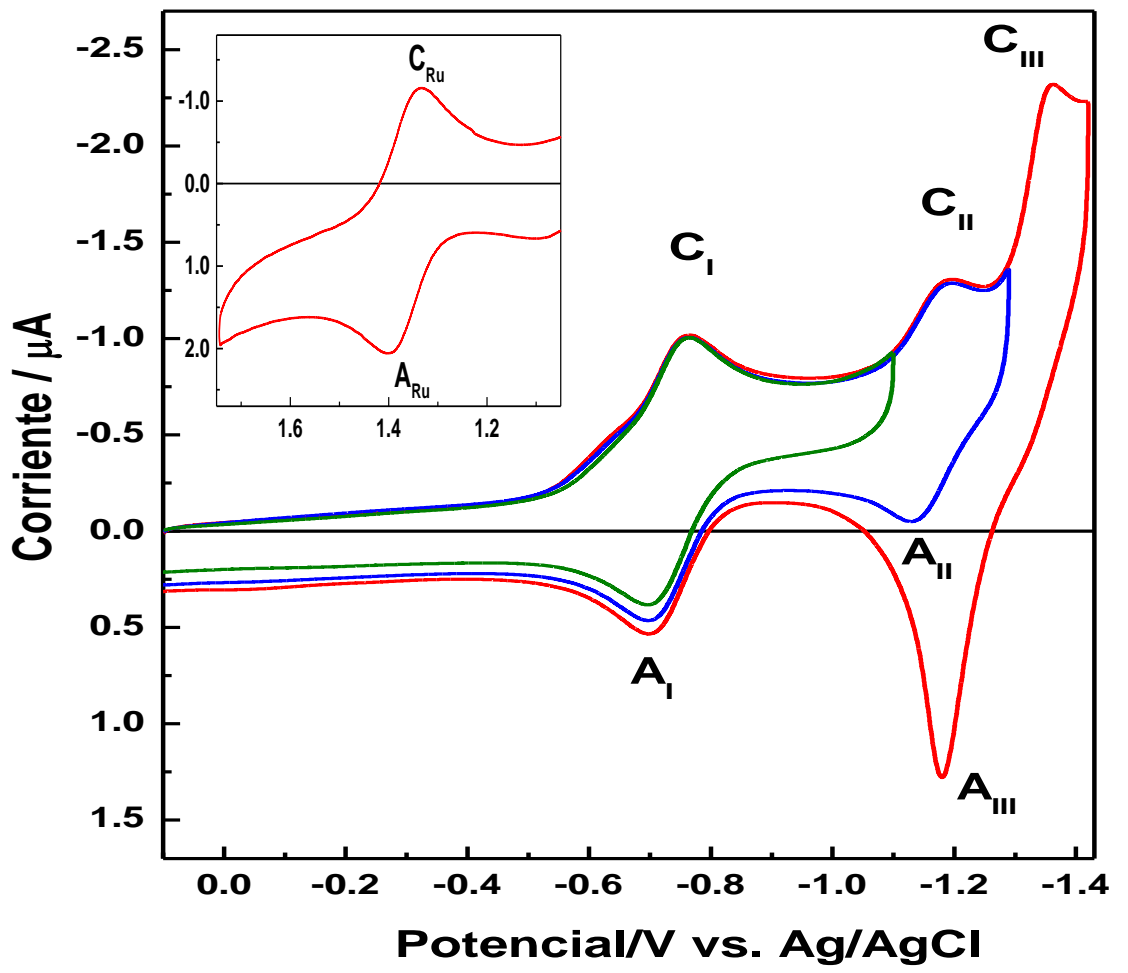

Figura 3. Voltamperometría cíclica de $\left[(\text { phen })_{2} R u(t a t p p \alpha) R u(\text { phen })_{2}\right]^{4+}\left(1^{+4}\right)$, en $\mathrm{MeCN}$, utilizando hexafluorofosfato de tetrabutilamonio como electrolito soporte $\left(\mathrm{TBAPF}_{6}\right)$.

En las figuras 3 y 4 se aprecian los perfiles de electroreducción del complejo $1^{4+}$, mientras que en el recuadro de la figura 3 se muestra la electrooxidación de Ru (II) en el mismo sistema. 
Los voltamperogramas presentados en la figura 3 muestran la reversibilidad tanto en la electroreducción del ligando tatpp $\alpha$ como en la electrooxidación del Ru (II) (recuadro). Se registraron tres voltamperogramas con límites catódicos de $-1.10 \mathrm{~V},-1.30 \mathrm{~V}$ y $-1.42 \mathrm{~V}$. Estos voltamperogramas permiten separar claramente 3 procesos sucesivos de transferencia de electrones en el complejo $1^{4+}$ indicados como $C_{\mid} / A_{I}, C_{\| I} / A_{\mid I}$ y $C_{\mid I I} / A_{\mid I I I}$ (donde $C$ hace referencia a los procesos catódicos y A a los procesos anódicos). El primer voltamperograma abarca el proceso $C_{l} / A_{l}$, mientras que el límite catódico para el segundo voltamperograma incluye al segundo proceso de reducción $C_{\|} / A_{\|}$. Cuando se extiende el potencial catódico a $-1.42 \mathrm{~V}$, se observa el tercer proceso de electroreducción $\mathrm{C}_{\| I I}$; el pico relacionado con $A_{\| I}$ es en realidad una onda que enmascara a $A_{\|}$debido a la proximidad de los potenciales para ambos picos. En efecto cada voltamperograma incluye la detección de una cupla redox (picos catódicos y anódicos relacionados). El primer proceso redox caracterizado como $C_{\mid} / A_{l}$ presenta un potencial de media onda $E_{1 / 2}=-0.73 \mathrm{~V}$, el segundo proceso $C_{\|}$ $/ A_{\|}$se asocia a $E_{1 / 2}=-1.14 \mathrm{~V}$ y el tercer proceso se encuentra en $E_{1 / 2}=-1.29 \mathrm{~V}$. En la parte superior (recuadro) de la figura 3 se observa el voltamperograma para la electrooxidación de $\mathrm{Ru}^{+2 /+3}$ del complejo $1^{4+}$ que presenta una onda reversible, situada en $\mathrm{E}_{1 / 2}=1.35 \mathrm{~V}$.

Tabla 1. Potenciales redox para $\mathbf{1}^{+4}, \mathbf{2}^{+4}$ y $\left[R u(\text { phen })_{3}\right]^{2+}$.

\begin{tabular}{|c|c|c|c|}
\hline \multirow[t]{2}{*}{ Complejo } & \multicolumn{2}{|c|}{ Potencial de reducción (V) } & \multirow{2}{*}{$\begin{array}{c}\text { Potencial de } \\
\text { oxidación (V) } \\
\mathrm{Ru}^{2+/ 3+}\end{array}$} \\
\hline & $1^{\mathrm{er}}$ & $2^{\text {do }}$ & \\
\hline $\begin{array}{c}{\left[(\text { phen })_{2} R u(\text { tatpp } \alpha) R u(\text { phen })_{2}\right]^{4+}} \\
\left(\mathbf{1}^{+4}\right) .\end{array}$ & -0.73 & -1.14 & 1.35 \\
\hline $\begin{array}{c}{\left[(\text { phen })_{2} \operatorname{Ru}(\operatorname{tatpp} \beta) R u(\text { phen })_{2}\right]^{4+}} \\
\left(\mathbf{2}^{+4}\right) .^{\mathbf{a}}\end{array}$ & -0.22 & -0.71 & 1.35 \\
\hline$\left[\mathrm{Ru}(\text { phen })_{3}\right]^{2+} . \mathbf{b}$ & -1.38 & -1.52 & 1.35 \\
\hline
\end{tabular}

Potenciales indicados vs $\mathrm{Ag} / \mathrm{AgCl}$, utilizando $\mathrm{TBAPF}_{6}$ como electrolito soporte en medio $\mathrm{MeCN}$. 
a (Konduri, R. et al., 2004, Tacconi, N. R. Lezna, R.O. et al., 2005)

b (Shreeyukta, S., Muñoz Zúníga, J. et al., 2011, Leventis, N. et al., 2004)

En la tabla 1 se pueden observar los potenciales de electroreducción y electrooxidación para los complejos de $\mathbf{1}^{4+}, \mathbf{2}^{4+}$ y $\left[\mathrm{Ru}(\text { phen })_{3}\right]^{2+}$. La primera reducción de $1^{4+3+}$ ocurre a un potencial $\sim 500 \mathrm{mV}$ más negativo que la observada para $2^{4+/ 3+}$, del mismo modo la segunda reducción de $1^{3+2+}$ se encuentra a un potencial $\sim 430 \mathrm{mV}$ más negativo que la de la cupla $2^{3+/ 2+}$. Podemos deducir que, como se diseñó en la preparación, la estructura tatpp $\alpha$ de $1^{4+}$ conduce a orbitales aceptores de mayor energía que los del ligando lineal tatpp $\beta$ de $2^{4+}$.

Los valores de potenciales de reducción, comparados en la tabla 1 confirman las propiedades aceptoras de electrones del ligando puente tatppa. Los ligandos fenantrolina axiales se reducen a potenciales más negativos (TokelTakvoryan, N. E. et al., 1973, Leventis, N. et al., 2004, Leveque, J. et al., 2005, Bracco, L. L. B., Muñoz Zúníiga, J. et al., 2011), los cuales no son explorados en la ventana de potencial de las diferentes medidas voltamperométricas realizadas.

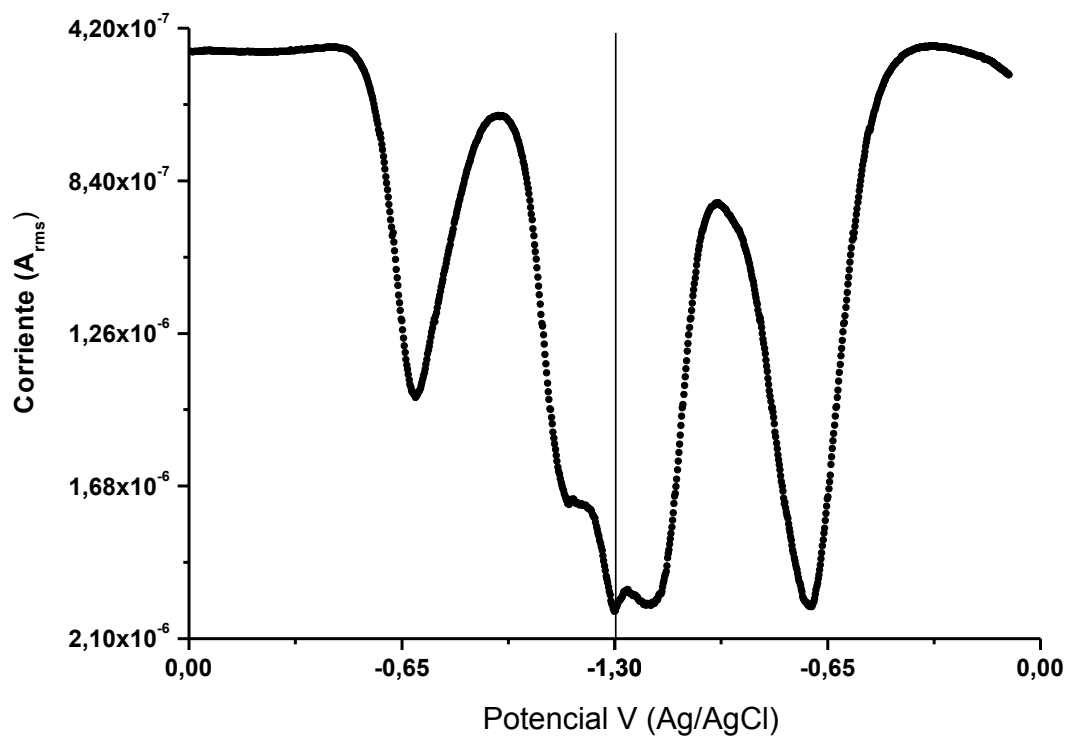

Figura 4. ACV del complejo $\left[(\text { phen })_{2} R u(\operatorname{tatpp} \alpha) R u(p h e n)_{2}\right]^{4+}\left(\mathbf{1}^{4+}\right)$, en $\mathrm{MeCN}$. Electrolito soporte TBAPF 6 . 
Utilizando la técnica de voltametría de alterna en el rango de medida de 0.0 a $-1.3 \mathrm{~V}$, se encuentran 2 picos correspondientes a la primera y segunda reducción de $1^{4+}$. Los valores de los potenciales de reducción - $0.69 \vee$ y $-1.15 \mathrm{~V}$ coinciden con los obtenidos inicialmente por voltametría cíclica y representan la formación de las especies $\mathbf{1}^{\cdot 3+}$ y $\mathbf{1}^{2+}$ respectivamente, indicando reducciones de 1 electrón. 


\subsection{Espectro electrónico de $1^{4+}$}

Para la interpretación de los resultados espectroelectroquímicos de $1^{4+}$ es importante tener una comprensión clara de su espectro de absorción. La figura 5 muestra los espectros electrónicos de los complejos dinucleares de $R u(I I), 1^{4+}$ y $2^{4+}$ en la figura 5 (a) y (b) respectivamente.

Los espectros de $1^{4+}$ presentan dos bandas intensas a 356 y $441 \mathrm{~nm}$, mientras que $2^{4+}$ se caracteriza por dos bandas principales situadas a 330 y 445 $\mathrm{nm}$. La banda de más baja energía (aproximadamente $441 \mathrm{~nm}$ ) en ambos complejos está asociada a una transición MLCT (metal-to-ligand charge-transfer) hacia las estructuras tipo fenantrolinas ubicadas en cada extremo del ligando central (phen - semejante). Fees y colaboradores han descrito a los orbitales "phen - semejante", como orbitales ópticos debido a su rol predominante en las propiedades ópticas del estado fundamental. (Fees, J. et al.,1993) .

La asignación de la banda MLCT se hizo por comparación con $\left[\mathrm{Ru}(\mathrm{phen})_{3}\right]^{2+}$. Diversos autores asignan bandas (con similar energía y forma) en aproximadamente $440 \mathrm{~nm}$ a la absorción de menor de energía de $\left[\mathrm{Ru}(\mathrm{phen})_{3}\right]^{2+}$, la cual es señalada como la transición MLCT Ru $(\mathrm{d} \pi) \rightarrow$ phen $\left(\pi^{\star}\right)$ que, a través de un cruzamiento entre sistemas, produce directamente el estado excitado triplete ${ }^{3} \mathrm{MLCT}$ responsable del comportamiento luminiscente de $\left[\mathrm{Ru}(\text { phen) })_{3}\right]^{2+}$ (Juris, $A$. et al., 1988, Kim, M-J. et al., 2002, Turro,C. et al., 1996). La banda MLCT para $2^{4+}$ es más aguda y estructurada que la banda plana ancha de $1^{4+}$ debido a contribuciones de transiciones centradas en el ligando (LC) de tatpp $\beta$ como lo muestra la figura $5(b)$. 


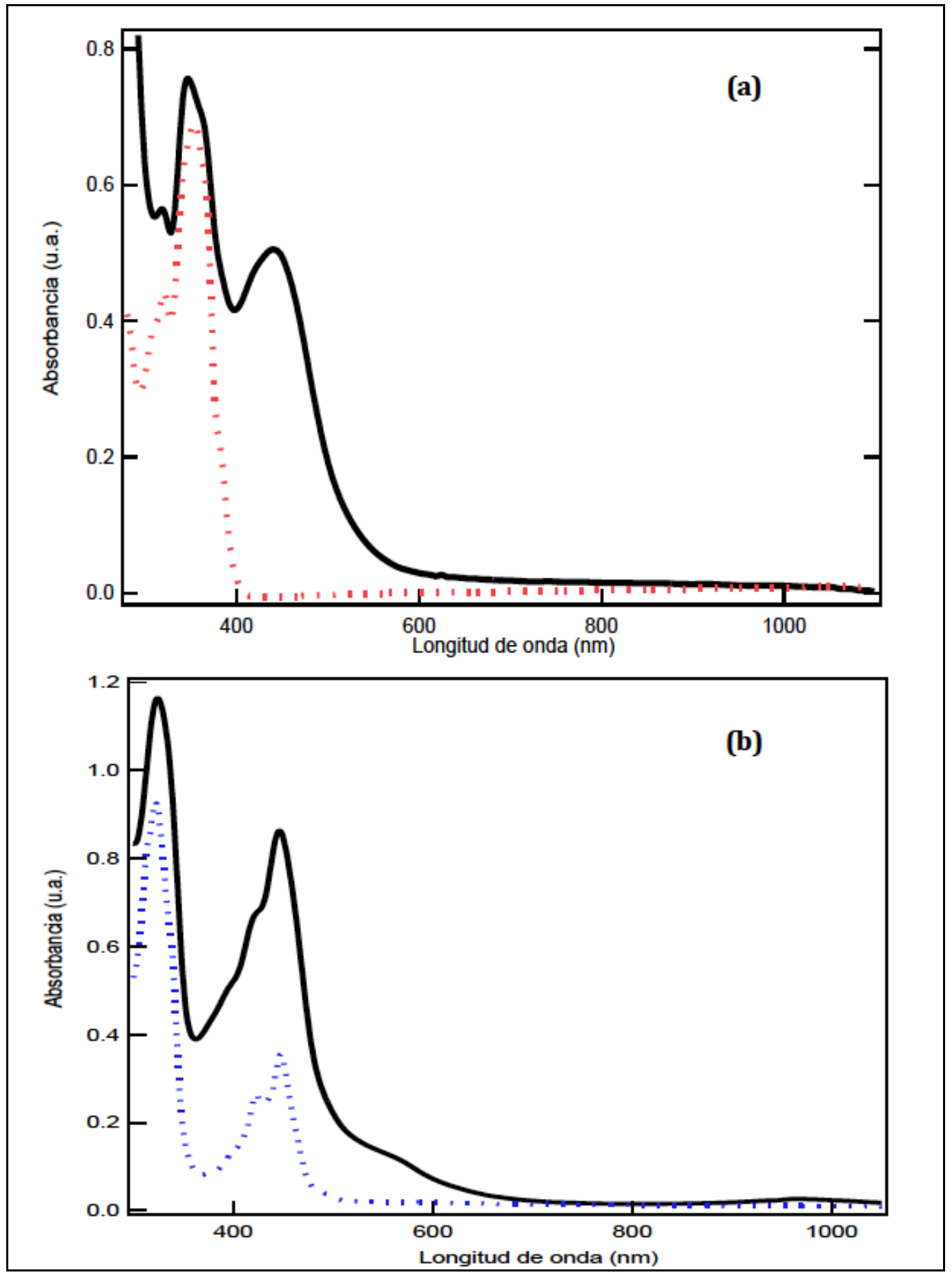

Figura 5. Comparación del espectro UV-Vis en MeCN de $20 \mu M$ de $1^{4+}$ (a) y $2^{4+}$ (b). Línea negra: espectro de cada complejo. Líneas punteadas: roja y azul: espectros del ligando tatpp $\alpha$ y tatpp $\beta$ respectivamente. 
La absorción espectral de los ligandos tatpp $\alpha$ y tatpp $\beta$ en $M e C N$, se observa en la figura 5 (a) y (b) respectivamente. Estos ligandos libres, son insolubles en este solvente y por lo tanto se hace necesario adicionar un exceso de iones Zn (II) para lograr su solubilización y obtener la banda de absorción del ligando central (LC) tanto en tatpp $\alpha$ como en tatpp $\beta$. Los complejos formados con Zn (II) están libres de transiciones MLCT (debido a la ausencia de Ru (II)). Aunque la coordinación con $\mathrm{Zn}$ (II) puede perturbar levemente algunas transiciones del ligando central (LC) (Wouters, K.L. et al., 2006), la magnitud de las perturbaciones observadas son pequeñas. En la figura 5 (b), el aducto $\mathrm{Zn}$ tatpp $\beta$ presenta dos absorciones intensas centradas en 325 y $445 \mathrm{~nm}$. El aducto $\mathrm{Zn}$ - tatpp $\alpha$ muestra un pico agudo en la misma región, centrado en $356 \mathrm{~nm}$, con un hombro en $321 \mathrm{~nm}$ figura 5 (a). Como se observa en la figura 5 (a), el complejo de rutenio $1^{4+}$ posee una transición MLCT ancha e intensa centrada en $441 \mathrm{~nm}$ y una transición LC de tatpp $\alpha$ a $356 \mathrm{~nm}$, esta última de similar intensidad y forma que la banda presente en el aducto de Zn (II). Una situación similar se observa en el espectro de $2^{4+}$, sin embargo en este caso, las transiciones LC a 445, 422 y $405 \mathrm{~nm}$ se solapan con la banda MLCT en $445 \mathrm{~nm}$, conduciendo a un espectro que resulta esencialmente la suma de las dos componentes. La banda LC tatpp $\beta$ a $325 \mathrm{~nm}$ se desplaza a $330 \mathrm{~nm}$ (para $2^{4+}$ ) y es ligeramente más intensa en $2^{4+}$ que en $\mathbf{1}^{4+}$, pero sin cambios de otro tipo (Shreeyukta, S.,Muñoz Zúñiga, J. et al., 2011).

El coeficiente de extinción molar $(\varepsilon)$ a $445 \mathrm{~nm}$ en MeCN para $\left[\mathrm{Ru}(\text { phen })_{3}\right]^{2+}$ es $19200 \mathrm{M}^{-1} \mathrm{~cm}^{-1}$ (Torres, A.S. et al., 1999) y para tatpp $\beta 17300 \mathrm{M}^{-1} \mathrm{~cm}^{-1}$; la suma de estos tres componentes, dos $\left[\mathrm{Ru}(\text { phen })_{3}\right]^{2+} \mathrm{y}$ un ligando tatpp $\beta$ resulta $\varepsilon_{445}=$ $55700 \mathrm{M}^{-1} \mathrm{~cm}^{-1}$ (Tacconi, N. R., Lezna, R.O. et al., 2005), ésta comparación concuerda con el coeficiente de extinción molar hallado para $2^{4+}, \varepsilon_{445}=65100 \mathrm{M}^{-1}$ $\mathrm{cm}^{-1}$ (Kim, M-J. et al., 2002) y se encontró que para el ligando tatppo el $\varepsilon_{445}$ corresponde a $1900 \mathrm{M}^{-1} \mathrm{~cm}^{-1}$, por lo tanto el coeficiente de extinción molar para $1^{4+}$ es $\varepsilon_{445}=40300 \mathrm{M}^{-1} \mathrm{~cm}^{-1}$. 


\subsection{Características de la forma oxidada y reducida de $1^{4+}$}

La reducción estequiométrica del complejo $1^{4+}$ con cobaltoceno en MeCN bajo condiciones anaeróbicas, se llevó a cabo con el fin de identificar las especies reducidas de $1^{4+}$ y conocer sus espectros correspondientes (figura 6).

El cobaltoceno es un agente reductor, con un potencial redox $\left[\mathrm{Co}(\mathrm{Cp})_{2}\right]^{+/ 0}$ de $-1.15 \mathrm{~V}$ vs $\mathrm{Ag} / \mathrm{AgCl}$ sat. $\mathrm{KCl}$ (Khanova, L. A. et al., 2006) capaz de reducir a $1^{4+}$, para generar $1^{\cdot 3+}$ y potencialmente $1^{2+}$ que corresponden a las especies observadas por voltametría en el primer y segundo potencial de reducción respectivamente ( -0.73 y $-1.14 \mathrm{~V}$ ); teniendo en cuenta la tabla 1 se puede deducir que ninguno de los dos potenciales señalados corresponde a la reducción en la phen del ligando axial (antena), ya que se ha encontrado que la reducción para $\left[\mathrm{Ru}(\text { phen })_{3}\right]^{2+} /\left[\mathrm{Ru}(\text { phen })_{3}\right]^{+}$ocurre a un potencial mucho más negativo $(-1.38$ V).

El ión cobaltoceno $\left[\mathrm{Co}(\mathrm{Cp})_{2}\right]^{+}$generado, generalmente tiene una solubilidad baja en solventes como el MeCN, posee un color amarillo muy claro ( $\lambda_{\max }=409$ $\mathrm{nm} ; \varepsilon=200)$; su presencia no interfiere apreciablemente con los espectros de absorción del visible e IR cercano (350 a 1100 nm). (Connelly, N. G. et al., 1996, Sohn, Y. S. et al., 1971).

La formación de la especie reducida $1^{\bullet 3+}$ por la adición de un equivalente de $\mathrm{Co}(\mathrm{Cp})_{2}$ se observa en la figura 6 (traza azul), la cual se caracteriza por la aparición de dos bandas en 460 y 505 nm, la desaparición de la banda a 356 nm y la presencia de una banda ancha de baja energía centrada en 
aproximadamente $930 \mathrm{~nm}$ es coherente con la formación de un anión radical con carga deslocalizada sobre el ligando tatpp $\alpha$.

El anión radical análogo $2^{\cdot 3+}$, también muestra picos anchos de energía baja a 855 y $965 \mathrm{~nm}$, los cuales representan una transición LC modulada por acoplamientos vibracionales. (Konduri, R. et al., 2004, Tacconi, N. R., Lezna, R.O. et al., 2005).

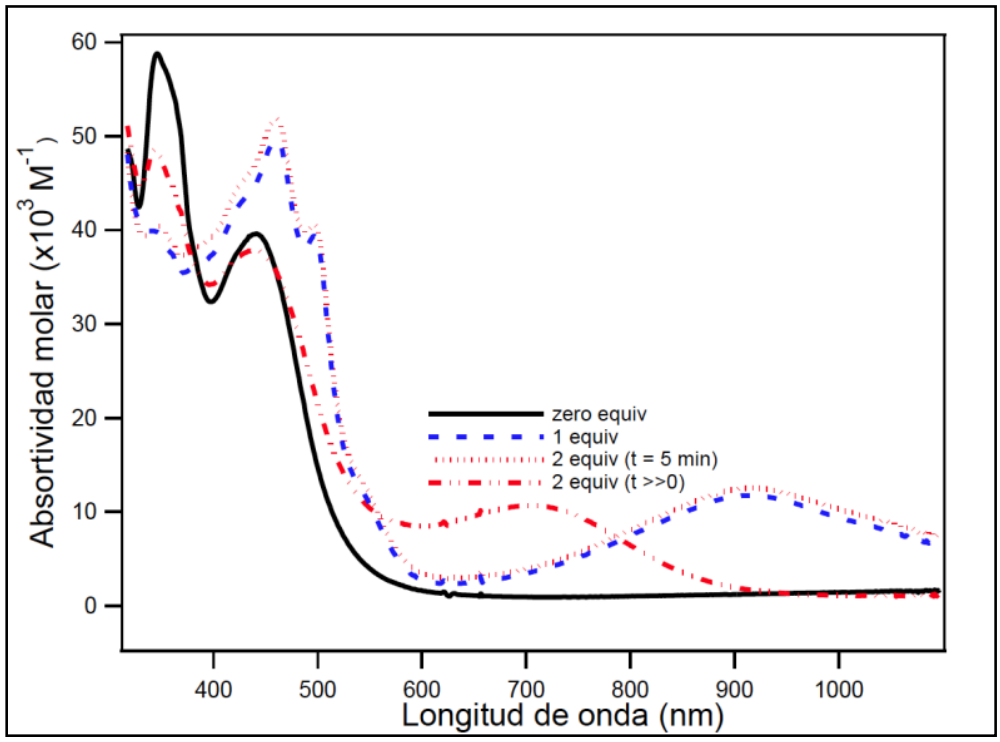

Figura 6. Evolución de la absorción espectral de $\mathbf{1}^{+4}$ bajo reducción química con cobaltoceno en $\mathrm{MeCN}$.

La adición de un segundo equivalente de $\mathrm{Co}(\mathrm{Cp})_{2}$, muestra inicialmente un aumento de las características espectrales vistas para $\mathbf{1}^{\mathbf{0}^{3+}}$ (figura 6 , trazas rojas). Sin embargo, cuando la solución se deja reposar durante toda la noche, se observa la aparición de nuevas bandas. Este nuevo espectro figura 6 (líneas y puntos rojos) muestra una banda ancha con un máximo en aproximadamente 700 nm, acompañado por la desaparición de bandas en 930, 460 y $505 \mathrm{~nm}$ (asociadas a la primera reducción del complejo $\left.\left(\mathbf{1}^{\bullet+}\right)\right)$. También se detecta una recuperación 
parcial de la banda de $356 \mathrm{~nm}$. Este cambio espectral es similar al observado para la formación de $\mathbf{2}^{2+}$ (Tacconi, N. R., Lezna, R.O. et al., 2005), por lo tanto, se puede relacionar con la especie doblemente reducida $1^{2+}$ a la que se asigna.

Se utilizaron agentes reductores más fuertes como el decametilcobaltoceno pero los resultados no aportaron información diferente. Como se verá más adelante, la lentitud puede deberse en parte a la dimerización del radical $\mathbf{1}^{\mathbf{3 +}}$ no observada para $2^{\bullet 3+}$.

Se han realizado medidas fotoquímicas para ${1^{4+}}^{4}$ en condiciones anaeróbicas utilizando trietilamina como reductor de sacrificio (Shreeyukta, S. , Muñoz Zúñiga, J. et al., 2011), los espectros de absorción resultantes durante la fotólisis son comparables a los presentados en la figura 6 para las dos reducciones estudiadas. 


\subsection{Espectroelectroquímica del complejo $\left[(\text { phen })_{2} \operatorname{Ru}(\text { tatpp } \alpha) \operatorname{Ru}(\text { phen })_{2}\right]^{4+}\left(1^{+4}\right)$}

La identificación espectroelectroquímica y el seguimiento de las especies que se generan durante la electrooxidación de $\mathrm{Ru}^{2+}$ a $\mathrm{Ru}^{3+}$ y la electroreducción del ligando puente central tatpp $\alpha$ de $\mathbf{1}^{4+}$, se realizaron mediante dos métodos complementarios, evolución temporal de los espectros de absorción UV-vis en los potenciales seleccionados y reflectancia diferencial $(\delta R / R)$ a longitudes de onda fijas.

Los cambios espectrales debido a la electrooxidación de los centros de rutenio en $\mathbf{1}^{4+}$ a $\mathbf{1}^{5+}$ y finalmente a $\mathbf{1}^{6+}$ se muestran en la figura 7 . El primer espectro corresponde a la línea negra sólida. Al aumentar el tiempo de electrólisis podemos ver un decrecimiento de la banda MLCT ( $445 \mathrm{~nm}$ ), esta desaparición es continua e indicativa de la oxidación simultánea de los dos centros de Ru (II). En el recuadro superior de la figura 3, se observa un solo pico para el proceso de electrooxidación, indicando que los dos centros de Ru (II) están débilmente acoplados. En el espectro también podemos ver la aparición de una nueva banda ancha a $690 \mathrm{~nm}$ que parece ser común en complejos de tris-diimina de $\mathrm{Ru}^{3+}$ (Gafney, H. D. et al., 1972, Elliott, C. M. et al., 1986, Wilson, R. A. et al.,2010). Finalmente, se observa que la banda LC a $356 \mathrm{~nm}$ aumenta en intensidad acompañada de un ligero corrimiento hacia $368 \mathrm{~nm}$.

Utilizando la técnica de reflectancia diferencial, se pudo obtener un panorama más detallado de los sistemas generados electroquímicamente a partir del complejo inicial $\left[(\text { phen })_{2} R u(\text { tatpp } \alpha) R u(\text { phen })_{2}\right]^{4+}\left(\mathbf{1}^{+4}\right)$. 


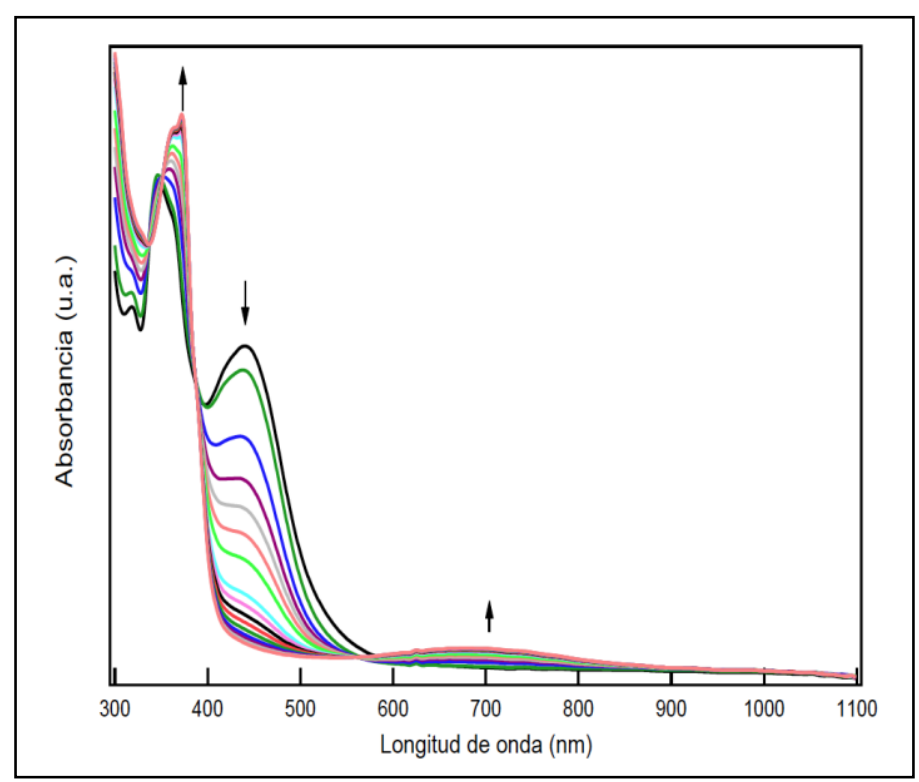

Figura 7. Espectroelectroquímica de la electrooxidación de $R u^{2+} \rightarrow R u^{3+}$ en $1^{4+}(80 \mu M)$ en MeCN y $0.1 \mathrm{M} \mathrm{TBAPF}_{6}$. Barrido de potencial de $0.0 \mathrm{Va}+1.6 \mathrm{~V}$ durante $180 \mathrm{~s}$. La adquisición de estos espectros se llevo a cabo en una celda capilar.

Los valores de absorción ( $\lambda \mathrm{s}$ ) obtenidos al realizar la caracterización de las especies reducidas químicamente con cobaltoceno provenientes de la figura 6 , se tomaron como referencias para llevar a cabo las medidas espectroelectroquímicas de reflectancia diferencial. Se realizaron medidas a las longitudes de onda de 850 $\mathrm{nm}, 460 \mathrm{~nm}$ y $650 \mathrm{~nm}$, elegidas para las especies formadas $\left(1^{\cdot 3+}\right.$ y $\left.1^{2+}\right)$ durante la electroreducción (figura 8 (a)). Los perfiles correspondientes a la figura 8 , muestran una imagen dinámica del destino del ingreso del primer y segundo electrón que ocurre a los potenciales de los picos $C_{\mid}$y $C_{\|}$respectivamente (figura 3). 

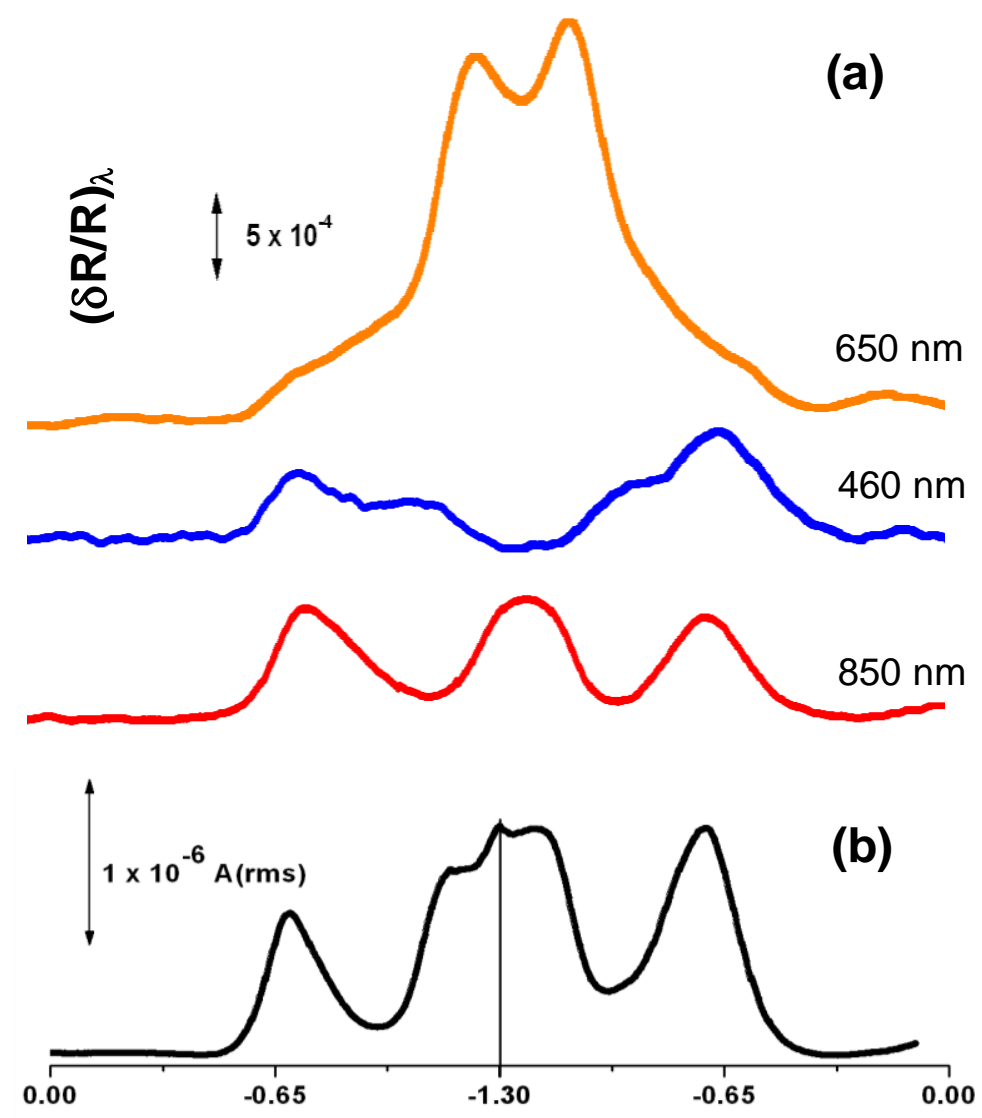

Potencial (V) ( $\mathrm{Ag} / \mathrm{AgCl})$

Figura 8. (a) Curvas $\delta R / R$ para $1^{+4}$ a $650 \mathrm{~nm}, 460 \mathrm{~nm}$ y $850 \mathrm{~nm}$ (b) ACV para $1^{4+}$. Ventana de potencial $=0.0 /-1.3 \mathrm{~V}$, concentración de $1^{4+}: 45 \mu \mathrm{M}$.

Estas medidas de $\delta \mathrm{R} / \mathrm{R}$ se realizaron empleando una onda sinusoidal de 50 $\mathrm{mV}_{\text {pk-pk }}$ (la mayor amplitud respecto a la usada para ACV se debe a la necesidad de generar una señal óptica, naturalmente mucho más pequeña que la correspondiente eléctrica, de magnitud medible), a una frecuencia de $6 \mathrm{~Hz}$ superpuesta a un barrido de potencial de $3 \mathrm{mV} / \mathrm{s}$. El voltamperograma ACV correspondiente a estas medidas, se muestra en la parte inferior, figura 8 (b). Los perfiles $\delta R / R$ para $850 \mathrm{~nm}$ y $460 \mathrm{~nm}$ se midieron para monitorear la especie generada $\left(1^{\bullet+3}\right)$ en la primera reducción de $1^{4+}$, mientras que con el perfil $\delta R / R$ a $650 \mathrm{~nm}$ se hace el seguimiento de la especie generada $\left(1^{2+}\right)$ en la segunda reducción de $1^{4+}$. Se ha elegido $650 \mathrm{~nm}$ y no $700 \mathrm{~nm}$ según la figura 6 para seguir 
la formación del complejo doblemente reducido $1^{2+}$, porque a esta longitud de onda hay menos solapamiento con el pico ancho de $930 \mathrm{~nm}$ propio de $1^{\bullet 3+}$.

Al realizar el estudio del sistema $\mathbf{1}^{{ }^{3+}}$ correspondiente al primer potencial de reducción $(-0.69 \mathrm{~V})$, la señal de $\delta \mathrm{R} / \mathrm{R}$ para $850 \mathrm{~nm}$ presenta una sola banda coincidente con el primer pico voltamétrico tanto en el barrido de ida como en el de regreso. El perfil de $\delta \mathrm{R} / \mathrm{R}$ para $460 \mathrm{~nm}$ muestra una señal que coincide con el de $850 \mathrm{~nm}$ seguido de un hombro que desaparece por completo al final del barrido (-1.3 V), este perfil es reversible.

Continuando con el análisis de la figura 8 , el perfil de $\delta R / R$ para $650 \mathrm{~nm}$ muestra la absorción del segundo electrón en -1.15 V. En este espectro también aparece un hombro en $-0.69 \mathrm{~V}$ proveniente de la primera reducción de $1^{4+}$. Lo mismo ocurre en el perfil $\delta R / R$ de 850 a potenciales más negativos (que $-0.69 \mathrm{~V}$ ), donde el aumento de su absorbancia es debido al crecimiento del pico ancho a $650 \mathrm{~nm}$ durante el proceso de formación de $\mathbf{1}^{2+}$.

Las figuras 9 (a) y (b) muestran el efecto de la frecuencia en las medidas de reflectancia diferencial $\delta R / R$ para la primera reducción de $1^{4+}$ (460 nm y 850 nm). En la figura 9 (c) se observa la voltametría de corriente alterna para el proceso de reducción de $1^{4+}$ correspondiente a la cupla $1^{4+/ 3+3+}$.

A las frecuencias de 6 y $11 \mathrm{~Hz}$ se puede notar que la intensidad de $\delta R / R$ aumenta inversamente proporcional a la frecuencia tanto para $850 \mathrm{~nm}$ (figura 9 (a)) como para $460 \mathrm{~nm}$ (figura 9 (b)). En el perfil de $\delta \mathrm{R} / \mathrm{R}$ a $850 \mathrm{~nm}$ se observa un solo pico en los barridos de ida y de vuelta a ambas frecuencias, mientras que $\delta \mathrm{R} / \mathrm{R}$ a $460 \mathrm{~nm}$ presenta asimetría en el pico correspondiente al barrido de ida y un pico ancho doble en el barrido de vuelta; esta respuesta puede ser indicativa 
de reacciones químicas posteriores. La desviación del comportamiento electroquímico ideal, presencia de un hombro, es más evidente a la frecuencia de modulación más baja, $6 \mathrm{~Hz}$ (figura 9 (b)).

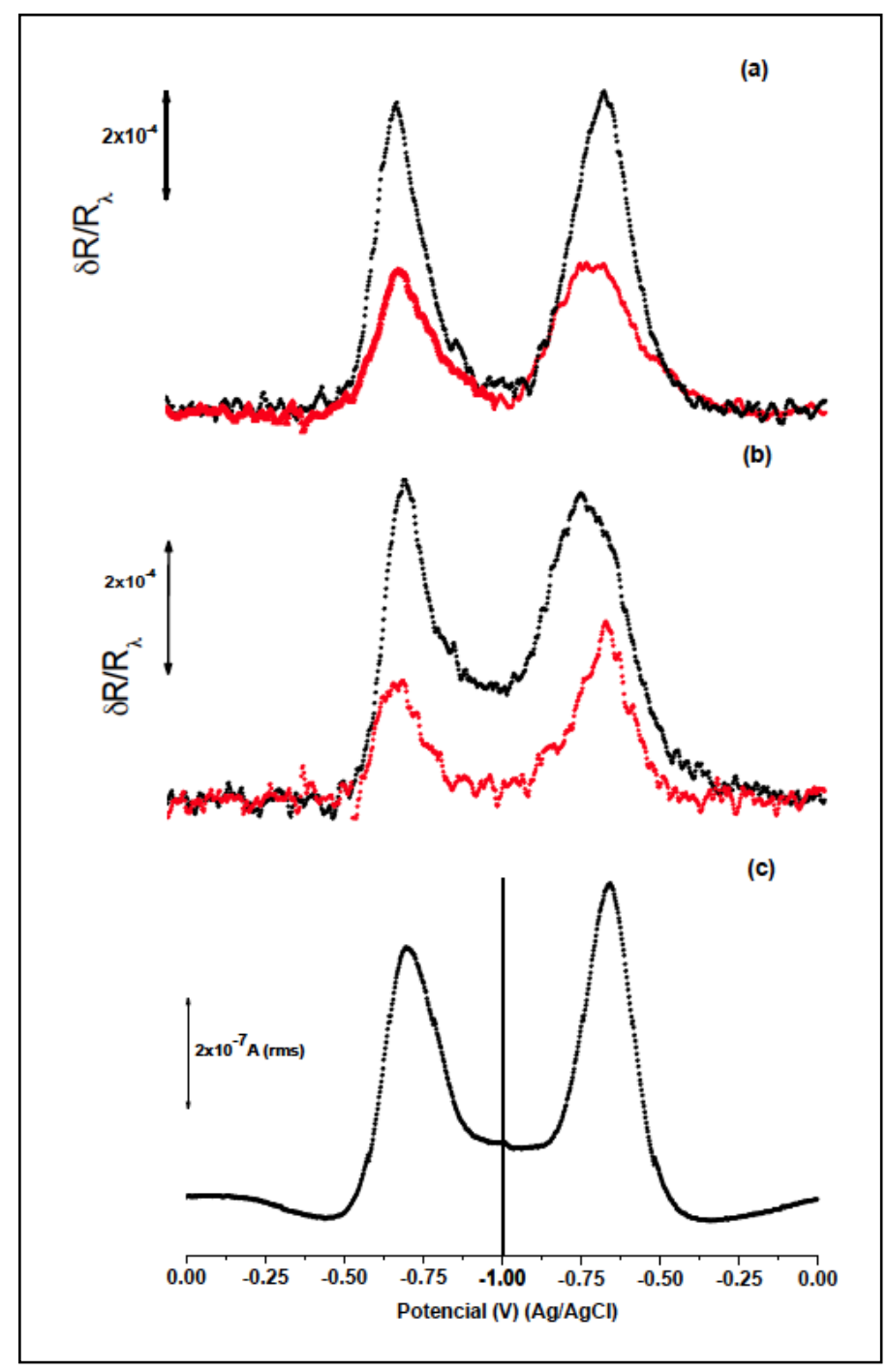

Figura 9. Efecto de la frecuencia de modulación sobre los perfiles $\delta R / R$ para el primer proceso de reducción de $\mathbf{1}^{4+}$. (a) $850 \mathrm{~nm}$ y (b) $460 \mathrm{~nm}$ (c) ACV para $\mathbf{1}^{4+}$. Los perfiles $\delta R / R$ para 6 y $11 \mathrm{~Hz}$ se muestran en trazas negro y rojo respectivamente. 


\subsection{Discusión}

Los isómeros $1^{4+}$ y $2^{4+}$, se diferencian en el patrón de sustitución sobre el anillo de benceno central en el ligando puente, conduciendo a una estructura "bent o doblada" con un ángulo de $120^{\circ}$ en $1^{4+}$, mientras $2^{4+}$ posee una estructura lineal (figura 2). Esta variación estructural es clave en las propiedades del estado fundamental. El primero y segundo potencial de reducción del ligando tatpp $\alpha$ en $\mathbf{1}^{4+}$ se desplazan a potenciales mucho mas negativos que para tatpp $\beta$ en $\mathbf{2}^{4+}$. Este resultado fue anticipado por los cálculos de OM, donde se muestra que la energía del LUMO en tapp $\alpha$ es sensiblemente mayor que para tatpp $\beta$. Es importante destacar que estos cálculos también indican que los orbitales aceptores centrales tienen energía más baja que los orbitales ópticos (phen - semejante), entre éstos se encuentran los orbitales de tatpp $\beta$ y de dppz, lo que sugiere que la capacidad de almacenamiento de múltiples electrones puede mantenerse a pesar de que los potenciales de reducción sean significativamente más negativos (Shreeyukta, S., Muñoz Zúñiga, J. et al., 2011).

Electroquímicamente los complejos $1^{4+}$ experimentan dos pasos reversibles de transferencia de un electrón cada uno, de la siguiente manera:

$$
\begin{array}{llll}
\mathbf{1}^{4+}+\mathrm{e}^{-} & \rightleftarrows & \mathbf{1}^{\cdot 3+} & \mathrm{E}_{1 / 2}(\mathrm{I})=-0.73 \mathrm{~V} \\
\mathbf{1}^{\cdot 3+}+\mathrm{e}^{-} & \rightleftarrows & \mathbf{1}^{2+} & \mathrm{E}_{1 / 2}(\mathrm{II})=-1.14 \mathrm{~V}
\end{array}
$$

Estos dos procesos son reversibles y bien separados en la escala de potenciales (figura 3 y 4 ). Se propone inicialmente el mecanismo de las reacciones 1 y 2, antes de incorporar la formación de un dímero al cual se hará referencia más adelante. 
La absorción espectral para los complejos $2^{4+}$ y $1^{4+}$, son similares. Ambos muestran una fuerte absorción MLCT en la región entre 440 - 480 nm típica para los complejos de Ru (II)-polipiridilo. La transición LC de más baja energía a 444 nm para $2^{4+}$ es coherente con la banda MLCT, y el espectro resultante es una sumatoria simple de las dos componentes. Esta transición LC es desplazada a $356 \mathrm{~nm}$ en $\mathbf{1}^{4+}$ y corresponde a un aumento de la energía de $0.69 \mathrm{eV}$ que coincide con el incremento calculado en la energía del LUMO, $0.64 \mathrm{eV}$ (Shreeyukta, S. et al., 2011). La banda LC de $444 \mathrm{~nm}$ de $\mathbf{2}^{4+}$, refleja la menor energía del LUMO en el ligando tatpp $\beta$. Esta banda también se manifiesta a potenciales de reducción más bajos asociados con el primer pico de electroreducción en $2^{4+}$ vs $1^{4+}$ (tabla 1 ).

La reducción química de $1^{4+}$ con cobaltoceno, muestra la formación de dos especies: $\mathbf{1}^{\mathbf{3 +}}$ y $\mathbf{1}^{2+}$, como se observa en la figura 5 . Al adicionar el primer equivalente de cobaltoceno se produce $\mathbf{1}^{\bullet+3+}$, mientras que la reducción subsiguiente para generar $\mathbf{1}^{2+}$ es más lenta en comparación al tiempo necesario para producir $\mathbf{2}^{2+}$ (Tacconi, N. R., Lezna, R.O. et al., 2005). No está clara la razón de la lentitud del proceso de formación de $\mathbf{1}^{2+}$, aunque podría deberse en parte a la dimerización del radical $\mathbf{1}^{\bullet+}$ como se verá más adelante.

El análisis de los datos espectroelectroquímicos de reflectancia diferencial

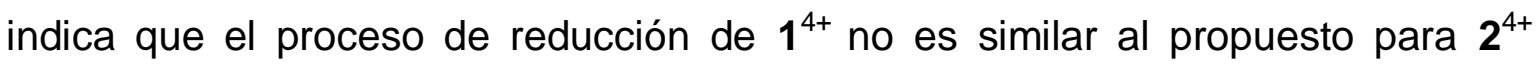
(Tacconi, N. R., Lezna, R.O. et al., 2005). En el caso de $1^{4+}$ se postula una dimerización del anión radical $\left(\mathbf{1}^{\bullet+}\right)$ para explicar las figuras 8 y 9 , que muestran a $1^{4+}$ experimentando dos procesos secuenciales reductivos cuasi reversibles a bajas velocidades de barrido y bajas frecuencias de modulación. La primera onda de reducción de $1^{4+}$ (pico $C_{l}$ del voltamperograma; figura 3 ) es dinámicamente seguida por $\delta \mathrm{R} / \mathrm{R}$ a 850 y $460 \mathrm{~nm}$ (figura 8 (a), 9 (a) y (b)) y la segunda reducción de $\mathbf{1}^{4+}$ (pico $C_{\|}$del ACV ; figura 3) por $\delta R / R$ a $650 \mathrm{~nm}$ (figura 8 (a)). 
Los perfiles de $\delta R / R$ a 460 y $850 \mathrm{~nm}$, muestran un pico que coincide con el del voltamperograma $A C$ correspondiente al ingreso del primer electrón $(-0.73 \mathrm{~V})$ (figura 9). A potenciales negativos del primer pico de reducción en el ACV de la figura 8 (b), la señal a $850 \mathrm{~nm}$ disminuye como se espera (figura 8 (a) inferior), sin embargo, la señal a $460 \mathrm{~nm}$ cae parcialmente pero luego muestra un pico ancho (dímero) que desaparece a -1.20 V (figura 8 (a) centro). El aumento de la señal a $850 \mathrm{~nm}$ a potenciales cercanos a $-1.3 \mathrm{~V}$ no es genuino, proviene de la superposición con la reducción siguiente, más negativa, que posee un pico ancho a $690 \mathrm{~nm}$, el que también se observa en la region de $850 \mathrm{~nm}$.

Se han propuesto los siguientes pasos mecanísticos basados en los perfiles de $\delta R / R$ en función del potencial. Esencialmente se trata de un mecanismo ECE (electroquímico-químico-electroquímico) como se indica a continuación:

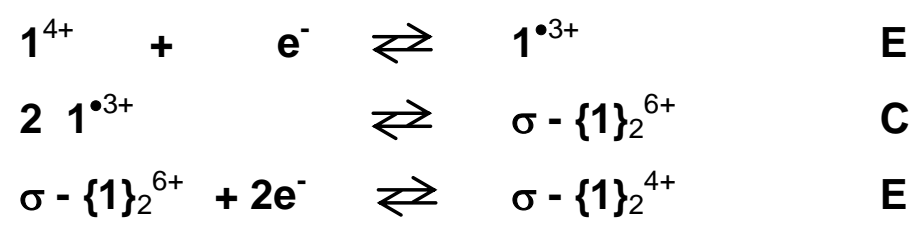

La reducción, mostrada en la reacción 3, es seguida por la dimerización de los radicales tatpp $\alpha$ (reacción 4) en un mecanismo tipo EC. La reacción química (4) involucra la dimerización de los dos radicales $1^{\cdot 3+}$ que forman un enlace $\sigma$ entre los ligandos centrales tatpp $\alpha$ formando $\sigma-\{1\}_{2}{ }^{6+}$. Podemos especular que la estructura "bent" ó doblada del ligando tatpp $\alpha$ favorece la formación del dímero uniendo los ligandos por sus cabezas, como se observa en la figura 10. Al parecer esta configuración no es estéricamente posible con el ligando lineal tatpp $\beta$ en $\mathbf{2}^{\mathbf{*}^{3+}}$. 


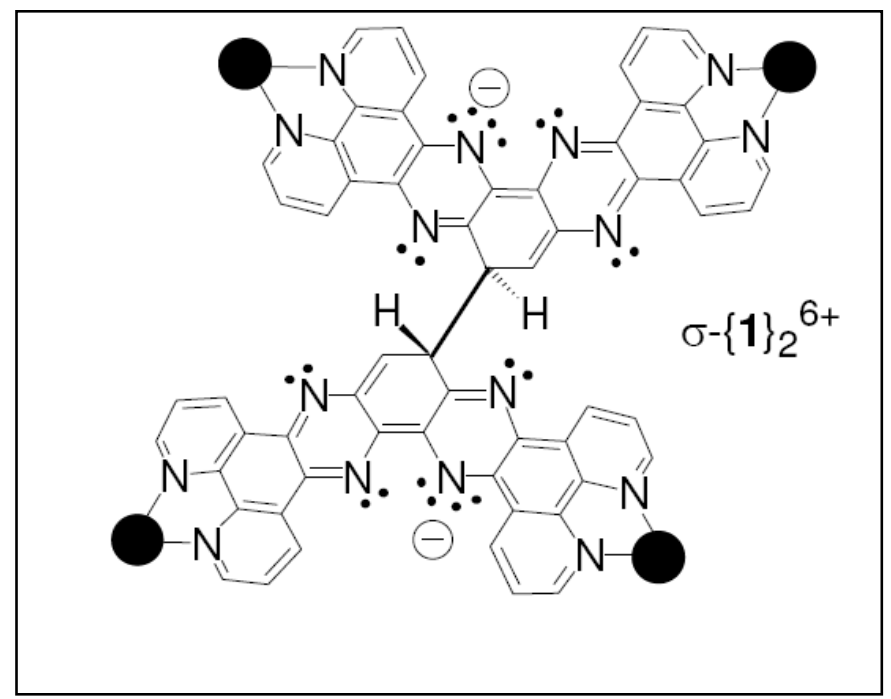

Figura 10. Estructura del dímero $\sigma-\{1\}_{2}^{6+}$ (los círculos sólidos representan los grupos $R u$ $\left.(\text { phen })_{2}^{2+}\right)$

La formación del dímero (C, reacción 4) sigue a la primera electroreducción (E, reacción 3) y es responsable del segundo pico observado a $460 \mathrm{~nm}$ en la figura 8 (a). El proceso de electroreducción siguiente $\left(\mathrm{C}_{\|} / \mathrm{A}_{\|}\right)$es asociado solamente con el mecanismo $\mathrm{E}$ (reacción 5) el cual involucra un electrón por complejo o dos electrones por dímero. La reacción 5 representa la reducción de dos electrones del nuevo dímero $\sigma-\{1\}_{2}{ }^{6+}$, en el que cada ligando tatpp $\alpha$ se reduce para producir $\sigma-\{1\}_{2}^{4+}$; este proceso aparece como un solo pico en el ACV y es acompañado por la señal a $650 \mathrm{~nm}$ figura 8 (a). Este paso de dimerización explicaría la lentitud en el segundo proceso de reducción. La formación del dímero indica que el anión radical tatppa es químicamente más reactivo que el anión radical relacionado en tatpp $\beta$.

El proceso extremadamente lento de reoxidación del producto doblemente reducido, respalda la propuesta de la formación de la estructura dimérica y, más importante aún, podría eventualmente limitar la utilidad del complejo para conducir reacciones multielectrónicas. Para evitar la dimerización que resulta en un 
proceso lento habría que buscar un ligando adecuado y un diseño apropiado de la estructura del complejo.

El desarrollo de fotocatalizadores capaces de impulsar reacciones multielectrónicas, por ejemplo, desprendimiento de hidrógeno, exige atenuar la formación de reacciones secundarias para prevenir dimerizaciones y evitar sustituyentes que sean buenos grupos salientes. 


\subsection{Conclusiones}

Los complejos ${1^{4+}}^{4} \mathbf{2}^{4+}$, son especies adecuadas para fotoimpulsar la reducción multielectrónica, sin embargo, cada uno muestra diferentes fortalezas y algunas debilidades respecto a su desempeño como fotocatalizadores funcionales. El ligando lineal tatpp $\beta$ de $2^{4+}$, muestra una reducción rápida, fácil y reversible de dos electrones como fue demostrado por métodos electroquímicos y/o fotoquímicos, sin embargo, el potencial de reducción de estos electrones almacenados no es suficientemente grande y parte de la energía absorbida en el proceso fotoquímico se disipa como calor.

El complejo $1^{4+}$ con tatpp $\alpha$ - doblado como ligando central, también presenta, de manera gradual, una reducción fotoinducida de dos electrones y los almacena a potenciales $500 \mathrm{mV}$ más negativos que $2^{4+}$. Un cálculo simple revela que el almacenamiento fotoreducido de $1^{2+}$ posee por lo menos $90 \mathrm{~kJ} / \mathrm{mol}$, más energía que $\mathbf{2}^{2+}$, sin embargo, la utilidad del proceso de fotoreducción en $\mathbf{1}^{4+}$ está en cierta manera acotada por las reacciones colaterales que conducen al radical anión dimerizado que limita su utilidad fotocatalítica. Para mejorar este aspecto se realizarán síntesis teniendo en cuenta los volúmenes estéricos para prevenir así reacciones secundarias indeseadas en los sitios probables de dimerización.

Es importante destacar que se ha demostrado que es posible aumentar significativamente la energía del orbital redox en el ligando aceptor tatpp $\alpha$ conservando la función aceptora multielectrónica en procesos fotoquímicos. El excelente acuerdo entre las propiedades redox observadas y las energías de orbitales calculadas por la teoría DFT de $1^{4+}$ y $\mathbf{2}^{4+}$ (Shreeyukta, S., Muñoz Zúñiga, 
J. et al., 2011), sugiere que los modelos de cálculo de distribuciones energéticas y electrónicas de los orbitales de estructuras potencialmente aceptoras, sean un medio útil y eficaz para anticipar resultados probables previos a la síntesis de laboratorio. 


\subsection{Referencias}

Amouyal, E.; Keller, P.; Moradpour, A. J. Chem. Soc., Chem.Commun. 1980, $1019-1020$.

Amouyal, E. Sol. Energy Mater. Sol. Cells 1995, 38, 249 - 276.

Barton, C. E.; Lakkaraju, P. S.; Rampulla, D. M.; Morris, A. J.; Abelev, E. y Bocarsly, A. B. J. Am. Chem. Soc. 2010, 132, 11539 - 11551.

Bracco, L. L. B.; Lezna, R. O.; Muñoz Zuñiga, J.; Ruiz, G.T; Féliz, M.R.; Ferraudi, G.J.; Einschlag García, F. S. y Wolcan, E. Inorganica Chimica Acta. 2011, 370, 482 - 491.

Campagna, S.; Puntoriero, F.; Nastasi, F.; Bergamini, G. y Balzani, V. Top. Curr. Chem. 2007, 280, 117 - 214.

Connelly, N. G. y Geiger, W. E. Chem. Rev. 1996, 96, 877 - 910.

Elliott, C. M. y Redepenning, J. G. J. Electroanal. Chem. 1986, 197, 219 232.

Elvington, M. y Brewer, K. J. Inorg. Chem. 2006, 45, 5242 - 5244.

Elvington, M.; Brown, J.; Arachchige, S. M. y Brewer, K. J. J. Am.Chem. Soc. 2007, 129, 10644 - 10645. 
Esswein, A. J.; Veige, A. S.; Nocera, D. G. J. Am. Chem. Soc. 2005, 127, $16641-16651$.

Fees, J.; Kaim, W.; Moscherosch, M.; Matheis, W.; Klima, J.; Krejcik, M.; Zalis, S. Inorg. Chem. 1993, 32, 166 - 174.

Gafney, H. D.; Adamson, A. W. J. Am. Chem. Soc. 1972, 94, 8238 - 39.

Ghosh, S.K.; Deb, B.M.; PHYSICS REPORTS (Review Section of Physics Letters) 1982, 92,1 - 44.

Hawecker, J.; Lehn, J-M.; Ziessel, R. New J. Chem. 1983, 7, 271.

Heyduk, A. F.; Nocera, D. G. Science 2001, 293, 1639 - 1641.

Holder, A. A.; Swavey, S.; Brewer, K. J. Inorg. Chem. 2004, 43, 303 - 308.

Juris, A.; Balzani, V.; Barigelletti, F.; Campagna, S.; Belser, P.; von Zelewsky, A. Coord. Chem. Rev. 1988, 84, 85 - 277.

Khanova, L. A.; Topolev, V.V; Krishtalik, L. I. Chemical Physics 2006, 326, 33 $-42$.

Kim, M-J.; Konduri, R.; Ye, H.; MacDonnell, F.M.; Puntoriero, F.; Serroni,S.; Campagna, S.; Holder, T.; Kinsel, G. y Rajeshwar, K. Inorg. Chem. 2002, 41, $2471-2476$.

Kirch, M.; Lehn, J-M.; Sauvage, J-P. Helv. Chim. Acta 1979, 62,1345 - 1384.

Konduri, R.; de Tacconi, N. R.; Rajeshwar,K. y MacDonnell, F. M. J. Am. Chem. Soc. 2004, 126, 11621 - 11629. 
Leventis, N.; Rawashdeh, A.-M. M.; Elder,I. A.; Yang,J.; Dass, A. y SotiriouLeventis, Ch. Chem. Mater. 2004, 16, 1493 - 1506.

Leveque, J.; Elias, B.; Moucheron, C.; y De Mesmaeker, A. K. Inorg. Chem. 2005, 44, 393 - 400.

Lezna, R. O.; de Tacconi, N. R.; Janaratne, T.; Muñoz Zúñiga, J. y MacDonnell, F. M. J. Argent. Chem. Soc. 2009, 97 (1), 273 - 288.

MacDonnell, F. M. In Solar Hydrogen Generation: Toward a Renewable Energy Future; Rajeshwar, K., Licht, S., Eds.; Springer Publishers: New York, 2008; Chapter 6.

Masayuki, K.; Shigeyuki, M.; Sakai, K. Molecules 2010, 15, 4908 - 4923.

Meyer, T. J. Acc. Chem. Res. 1989, 22, 163 - 169.

Molnar, S. M.; Nallas, G.; Bridgewater, J. S.; Brewer, K. J. J. Am. Chem. Soc. 1994, 116, 5206 - 5210.

Nagy, Á. Physics Reports 1998, 298, 1 - 79.

Ozawa, H.; Haga, M. A.; Sakai, K. J. Am. Chem. Soc. 2006, 128, 4926 - 4927.

Pfennig, B. W.; Lockard, J. V.; Cohen, J. L.; Watson, D. F.; Ho, D. M.; Bocarsly, A. B. Inorg. Chem. 1999, 38, 2941 - 2946.

Pfennig, B. W.; Mordas, C. J.; McCloskey, A.; Lockard, J. V.; Salmon, P. M.; Cohen, J. L.; Watson, D. F.; Bocarsly, A. B. Inorg. Chem. 2002, 41, 4389 4395. 
Rau, S.; Schafer, B.; Gleich, D.; Anders, E.; Friedrich, M.; Gorls, H.; Henry, W. y Vos, J. G. Angew. Chem., Int. Ed. 2006, 45, 6215 - 6218.

Rosenthal, J.; Bachman, J.; Dempsey, J. L.; Esswein, A. J.; Gray, T. G.; Hodgkiss, J. M.; Manke, D. R.; Luckett, T. D.; Pistorio, B. J.; Veige, A. S. Y Nocera, D. G. Coord. Chem. Rev. 2005, 249, 1316 - 1326.

Shreeyukta, S.; de Tacconi, N.R.; Diaz, N.R.G.; Lezna, R. O.; Muñoz Zuñiga, J.; Abayan, K. y MacDonnell, F.M. Inorg. Chem. 2011, 50, (19), 9318 - 9328.

Sohn, Y. S.; Hendrickson, D.N. y Gray, H. B. J. Am. Chem. Soc. 1971, 93,(15),3603 - 3612.

Tacconi, N. R.; Lezna, R. O.; Konduri, R.; Ongeri, F.; Rajeshwar, K. y MacDonnell, F. M. Chem. Eur. J. 2005, 11, 4327 - 4339.

Tacconi, N. R.; Chitakunye, R.; MacDonnell, F.M. y Lezna, R. O. J. Phys. Chem. 2008 a, 112, 497 - 507.

Tacconi, N. R.; Lezna, R. O.; Chitakunye, R. y MacDonnell, F. M. Inorg. Chem. 2008 b, 47, 8847 - 8858.

Tokel-Takvoryan, N. E.; Hemingway, R. E. y Bard, A. J. J. Am. Chem. Soc. 1973, 95 (20), 6582 - 6589.

Torres, A.S.; Maloney, D.J.; Tate, D.; Saad, Y. y MacDonnell, F.M. Inorg. Chim. Acta. 1999, 293, 37- 43.

Turro, C.; Chung,Y. C.; Leventis,N.; Kuchenmeister, M. E.; Wagner, P.J. y Leroi, G. E. Inorg. Chem. 1996, 35, 5104 - 5106.

Watts, R. J. Comm. Inorg. Chem. 1991, 11, 303 - 337. 
Wilson, R. A.; Pinyayev, T. S.; Membreno, N. y Heineman, W. R. Electroanalysis 2010, 22, 2162 - 2166.

Wouters, K. L.; de Tacconi, N. R.; Konduri, R.; Lezna, R. O. y MacDonnell, F. M. Photosynth. Res. 2006, 87, 41 - 55. 


\section{Reducción reversible}

multielectrónica del 9,11,20,22tetraaza-tetrapiridopentaceno

(tatpp) como ligando axial en el complejo [(bpy) ${ }_{2} \mathrm{Ru}($ tatpp) $] \mathrm{Cl}_{2}$ 
Reducción reversible de tatpp en $\mathbf{3}^{2+} \mid 2$ Capítulo 4. 
Capítulo 4. Reducción reversible multielectrónica del 9,11,20,22- tetraazatetrapiridopentaceno (tatpp) como ligando axial en el complejo $\left[(\text { bpy })_{2} \mathrm{Ru}(\right.$ tatpp $\left.)\right] \mathrm{Cl}_{2}$.

4.1 Introducción

4.2 Parte experimental

4.3 Electroquímica de $\left[(\text { bpy })_{2} R u(\text { tatpp })\right]^{2+}\left(3^{2+}\right)$ y del aducto $Z n$-tatpp

4.4 Espectro de absorción para $3^{2+}$ y de las especies relacionadas formadas químicamente.

4.5 Espectroelectroquímica del complejo $\left[(\text { bpy })_{2} R u(\text { tatpp })\right]^{2+}\left(3^{+2}\right)$ y del aducto $\mathrm{Zn}$-tatpp.

4.6. Discusión

4.7 Mecanismo de electroreducción

4.8. Conclusiones

4.9 Referencias. 


\subsection{Introducción}

En los últimos años, se ha mostrado interés en fotocatalizadores moleculares diseñados para el uso de energía solar para generar $\mathrm{H}_{2}$ y/o convertir el $\mathrm{CO}_{2}$ en combustibles útiles como metanol y metano (Sun, L. et al., 2001, Balzani, V. et al., 2003, Alstrum-Acevedo, J. H. et al., 2005, Dempsey, J. L. et al., 2005, Eisenberg, R. et al., 2005, Armaroli, N. et al., 2007). Hasta la fecha, sólo unos pocos fotocatalizadores artificiales han mostrado capacidad de almacenamiento electrónico múltiple, reductor u oxidante, en un proceso fotoquímico (Molnar, S. M. et al., 1994, Chang, C. C. et al., 2000, Heyduk, A. F. et al., 2001, Konduri, R. et al., 2002, Pfennig, B. W. et al., 2002, Borgstrom, M. et al., 2005, Esswein, A. J. et al., 2005, Rosenthal, J. et al., 2005, Elvington, M. et al. 2006) a pesar de que estas funciones son esenciales para los fotosistemas en la naturaleza. La transferencia de protones es también una característica esencial de procesos fotoinducidos que acompaña a la transferencia electrónica en procesos de almacenamiento de energía para sistemas naturales (Kirmaier, K. et al., 1988, Tommos, C. et al., 1998). Del mismo modo, la transferencia de protones desempeña un papel importante en la fotocatálisis artificial. Por todo lo dicho, es necesario un mejor entendimiento de los mecanismos de procesos multielectrónicos acompañados por protonación (Cukier, R. I. et al., 1998, Huynh, M. H. et al., 2007).

Se ha demostrado que los complejos dinucleares de rutenio (II), $\left[(\text { phen })_{2} R u(\text { tatpp }) R u(\text { phen })_{2}\right]^{4+} \quad\left(2^{4+}\right)$ y $\quad\left[(\text { bpy })_{2} R u(\text { tatpp }) R u(\text { bpy })_{2}\right]^{4+} \quad\left(4^{4+}\right)$, son fotoquímicamente activos y presentan dos reducciones en el ligando tatpp bajo irradiación con luz visible en presencia de donores de sacrificio (Konduri, $R$. et al., 2002, 2004, de Tacconi, N. R., Lezna, R. O. et al., 2005). El complejo con 
fenantrolina ha mostrado también actividad biológica ya que su forma doblemente reducida se une al ADN por un mecanismo que parece incluir un radical intermediario centrado en el carbono (Janaratne, T. K. et al., 2007). Los complejos mononucleares relacionados, $\quad\left[(\text { bpy }){ }_{2} R u(\text { tatpp })\right]^{2+} \quad\left(3^{2+}\right)$ y $\left[(\text { phen })_{2} \mathrm{Ru} \text { (tatpp) }\right]^{2+}$ muestran procesos redox similares en MeCN. Sin embargo, la química redox es complicada debido a la formación espontánea de estructuras conectadas por uniones $\pi$ (facilitadas por la superposición de ligandos tatpp planos) en MeCN, así como la formación de dímeros $\sigma$ entre las especies de radicales (Tacconi, N. R., Lezna, $R$. O. et al., 2008a).

El proceso de electroreducción para $\mathbf{3}^{2+}$ en solución acuosa no conduce a agregación ni a dimerización de radicales, mientras que cuando se utiliza $\mathrm{MeCN}$, el complejo permanece en gran medida desagregado y por lo tanto es más fácil examinar tanto la protonación como los detalles mecanísticos de electroreducción basados en el ligando tatpp (Tacconi, N. R. , Lezna, R. O. et al., 2008b).

En este capítulo, se comparan los procesos de reducción multi-electrónica del complejo $\mathbf{3}^{2+}$ y del aducto $Z$ n-tatpp, mostrados en la figura 1. El ligando tatpp libre es insoluble en todos los solventes comunes y la coordinación con $\mathrm{Zn}^{2+}$ es necesaria para la solubilización. En estas condiciones de exceso de $\mathrm{Zn}^{2+}$, lo más probable es que ambos extremos estén coordinados a los iones $\mathrm{Zn}^{2+}$, sin embargo, por simplicidad, nos referimos al aducto como Zn-tatpp. 


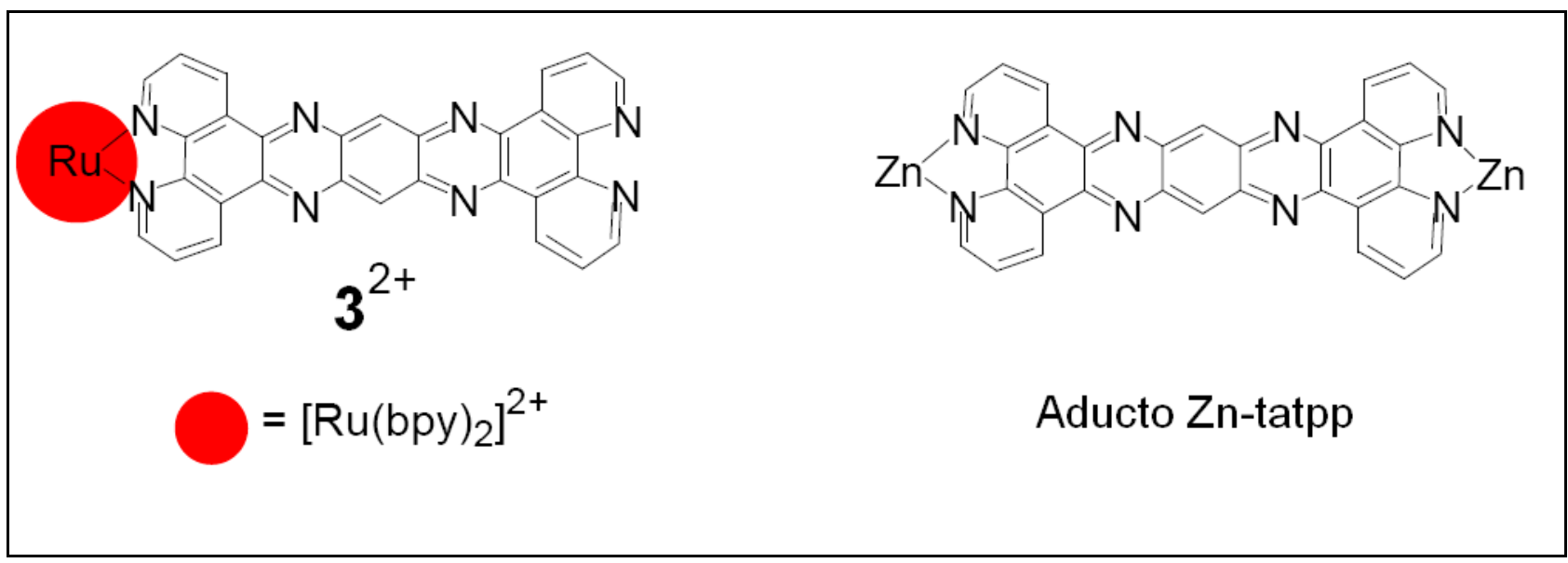

Figura 1. Estructura de $\left[(\text { bpy })_{2} R u(\text { tatpp })\right]^{2+}, 3^{2+}, y$ del aducto Zn-tatpp

Se espera que las propiedades ópticas de la molécula tatpp no cambien de manera significativa cuando esté coordinado al $\mathrm{Zn}^{2+}$, aunque probablemente resulte perturbado el potencial redox del ligando tatpp, fundamentalmente a través de interacciones electrostáticas. Por el contrario, la coordinación de tatpp a $\mathrm{Ru}^{2+}$ da lugar a una interacción mucho más covalente, además de electrostática, que conduce a perturbaciones en los procesos redox del ligando tatpp. La espectroelectroquímica se utilizó para determinar las especies formadas durante la captación (reducción) multielectrónica reversible en ambos sistemas. Para el análisis de las especies generadas durante la electroreducción de $\mathbf{3}^{2+}$, se eligió una solución acuosa básica para evitar la dimerización del radical, mientras que para la solubilidad del aducto de $\mathrm{Zn}$-tatpp, se utilizó una mezcla $\mathrm{MeCN}$ : $\mathrm{H}_{2} \mathrm{O}$ con exceso molar, 10X, de $\mathrm{Zn}^{2+}$ por tatpp. En ambos casos, se demuestra la incorporación reversible de cuatro electrones, con diferentes mecanismos que fueron resueltos con la ayuda de mediciones espectroelectroquímicas. 


\subsection{Parte Experimental}

Como electrodo de trabajo se utilizó carbón vítreo Cypress $(1.5 \mathrm{~mm}$ de diámetro de disco). Antes de su uso, el electrodo se pule a espejo utilizando alúmina húmeda (Buehler, $0.05 \mathrm{~m}$ ), a continuación se enjuaga con agua Millipore Milli-Q y se sonica. Para el complejo $3^{2+}$, las soluciones de electrolito fueron preparadas a partir del buffer $\mathrm{NaH}_{2} \mathrm{PO}_{4} / \mathrm{K}_{2} \mathrm{HPO}_{4}$, el pH se ajustó con $\mathrm{NaOH} 0.5$ M; mientras que para la molécula tatpp, se utilizó tetrabutilamonio hexafluorofosfato $\left(\mathrm{TBAPF}_{6}\right) \quad 0.1 \mathrm{M}$ como electrolito soporte en una mezcla de $\mathrm{MeCN}: \mathrm{H}_{2} \mathrm{O} /$ 90:10. Antes de cada medida, las soluciones fueron desoxigenadas con burbujeo de argón y se mantuvo esta atmósfera sobre la solución electroquímica durante todo el transcurso del experimento. Todos los experimentos se realizaron a la temperatura ambiente de laboratorio (ca. $20 \pm 2^{\circ}$ C).

Tanto el cobaltoceno $\left(\mathrm{Co}(\mathrm{Cp})_{2}\right)$ como el ácido trifluoroacético (TFA), (ambos de Alfa Aesar), se emplearon sin purificación adicional. Las titulaciones redox y de protonación se llevaron a cabo en una caja seca en atmósfera de nitrógeno, utilizando soluciones estándar de $3\left[\mathrm{PF}_{6}\right]_{2}\left(10^{-4} \mathrm{M}\right), \mathrm{Co}(\mathrm{Cp})_{2}(0.01 \mathrm{M})$, y TFA (0.01 M). Los compuestos $3^{\circ+}$ y $3^{0}$ fueron generados por la adición de 1 y 2 equivalentes de $\mathrm{Co}(\mathrm{Cp})_{2}$, respectivamente. Las especies protonadas se generaron por la adición estequiométrica de TFA a las soluciones de $3^{\bullet+}$ y $3^{0}$.

Para las determinaciones espectroelectroquímicas de UV-visible fueron utilizados dos configuraciones: 
(a) Las mediciones de absorbancia espectroelectroquímica se realizaron a potencial constante en una cubeta de $4 \mathrm{~mm}$, con una película semitransparente de óxido de indio dopado con estaño (ITO) sobre un sustrato de vidrio como electrodo de trabajo y se presentan como diferencias de espectros de absorción, $\Delta \mathrm{A}$ (espectros integrales), para describir los cambios espectrales que ocurren en la interfase electrodo/electrolito. Los datos espectroelectroquímicos se registraron en función del tiempo mediante el uso de un espectrómetro convencional de diodos (Hewlett-Packard modelo 8453). El contra electrodo (alambre de platino) y el microelectrodo de referencia de $\mathrm{Ag} / \mathrm{AgCl}$ (Cypress EE009) fueron colocados lateralmente en la cubeta de cuarzo.

(b) Las mediciones de reflectancia, utilizando un electrodo de oro pulido a espejo, fueron realizadas con un OMA, durante un barrido lento de potencial $(<5 \mathrm{mV} / \mathrm{s})$, los datos se presentan como $\Delta R / R$ vs potencial a longitudes de onda fijas, seleccionadas. Los cambios de reflectancia, $\Delta R=$ $\mathrm{R}-\mathrm{R}_{0}$, se miden con respecto a la reflectancia del sistema en un potencial $\left(R_{0}\right)$, donde no hay reacciones (típicamente región de doble capa). 


\subsection{Electroquímica de $\left[(\mathrm{bpy})_{2} \mathrm{Ru}(\operatorname{tatpp})\right]^{2+}\left(3^{2+}\right)$ y del aducto $\mathrm{Zn}$-tatpp}

Los voltamperogramas representativos de la electroreducción del complejo $3^{2+}$ y del aducto $\mathrm{Zn}$-tatpp se muestran en la figura 2. Para el complejo $3^{2+}$, la figura 2a compara el efecto del solvente acetonitrilo vs. agua a un $\mathrm{pH}$ de 10.5 , en los perfiles voltamperométricos obtenidos con un electrodo de carbón vítreo. Los dos principales procesos redox, $C_{\mid} / A_{\mid}$y $C_{\|} / A_{\|}$están aproximadamente en los mismos potenciales, para ambos solventes a -0.41 y $-0.81 \mathrm{~V}$, respectivamente, sin embargo, el pico $C_{\mid} / A_{l}$ se define mejor en medio acuoso, mientras que en acetonitrilo se detectan dos contribuciones superpuestas de dímero-monómero descriptas en Tacconi, N. R., Lezna, R. O. et al., 2008a. A potenciales más negativos que -1.2. $\mathrm{V}$ se observan los procesos redox reversibles relacionados con el ligando bipiridilo, estos picos no son accesibles en medio acuoso debido a la interferencia de la reacción de desprendimiento de hidrógeno. Para el aducto Zn-tatpp, también se observan dos procesos redox en $0.02 \mathrm{~V}$ y $-0.30 \mathrm{~V}$, respectivamente (figura $2 \mathrm{~b}$ ), los que se encuentran a potenciales significativamente más positivos que en el complejo $3^{2+}$. A potenciales más negativos se observa una cupla adicional de óxido-reducción ( $C^{\prime} / A^{\prime}$ ) que se relaciona con la reducción/(stripping) de $\mathrm{Zn}\left(\mathrm{Zn}^{2+} / \mathrm{Zn}^{0}\right)$ ya que $\mathrm{Zn}(\mathrm{BF} 4)_{2}$ se encuentra en exceso de concentración. Teniendo en cuenta que los dos primeros procesos redox en el complejo $3^{2+}$ (figura $2 \mathrm{a}$ ) y el aducto $\mathrm{Zn-tatpp} \mathrm{(figura} 2 \mathrm{~b}$ ) se asocian a la electroreducción del ligando tatpp, los potenciales más positivos en el aducto $\mathrm{Zn}$-tatpp revelan una menor deslocalización de electrones sobre el ligando tatpp en el caso del $\mathrm{Zn}^{2+}$ en relación al $\mathrm{Ru}^{2+}$, consistente con una fuerte retrodonación $\pi$ en el complejo de $\mathrm{Ru}$. Ciclando repetidamente el potencial entre los límites $0.5 \mathrm{~V}$ a $-0.8 \mathrm{~V}$, el aducto de $\mathrm{Zn}$ forma una película insoluble sobre la superficie del electrodo como se observa en la figura 3 . En los primeros 15 ciclos 


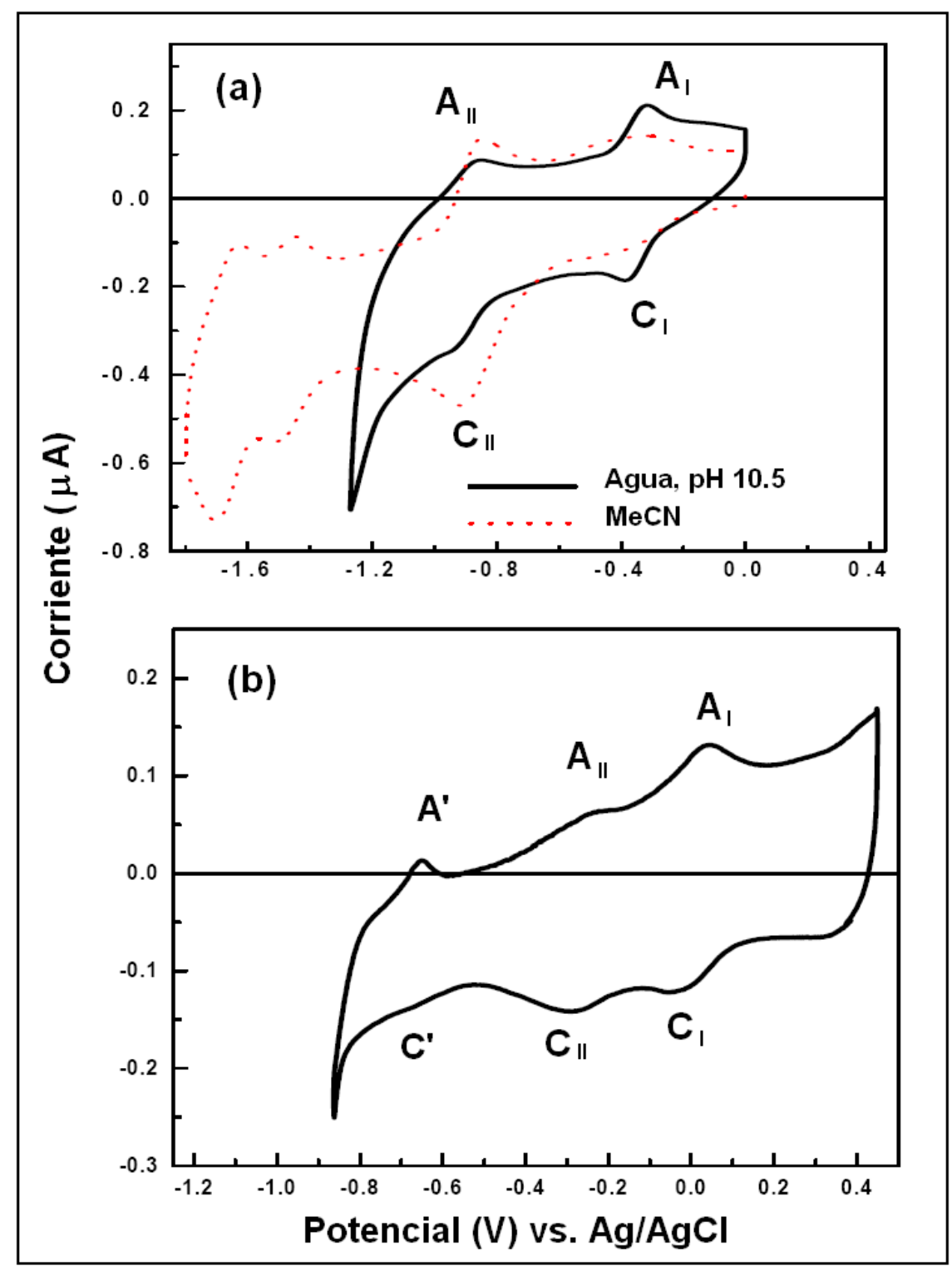

Figura 2. (a) Voltamperogramas a $20 \mathrm{mV} / \mathrm{s}$ para la electroreducción - electrooxidación del complejo $3^{2+}(20 \mu \mathrm{M})$, en medio acuoso ( $p \mathrm{H}$ 10.5) (línea negra sólida hasta -1.3 V) y en $\mathrm{MeCN}$ (línea roja punteada hasta $-1.8 \mathrm{~V}$ ). (b) Voltamperograma a $40 \mathrm{mV} / \mathrm{s}$ para la electroreducción/ electrooxidación de Zn-tatpp (25 $\mu \mathrm{M})$

(solamente se muestran los ciclos 1,7 y 15 para mayor claridad), hay un aumento progresivo de la corriente en los dos procesos redox hasta ca. 20 ciclos para luego disminuir dejando la superficie del electrodo cubierta por una película azul. 
El cambio gradual negativo en el potencial de reducción catódico sugiere que algunos $\mathrm{Zn}^{2+}$ se pierden en el proceso ya que la eliminación de $\mathrm{Zn}^{2+}$ modifica negativamente al potencial. La eficacia del crecimiento de la película, medida por el aumento de la corriente de pico, se ha encontrado que depende tanto del exceso de $\mathrm{Zn}^{2+}$ como de la concentración del electrolito soporte TBAPF 6 . La disminución de la concentración de 0.1 a $0.03 \mathrm{M}$ en $\mathrm{TBAPF}_{6}$, reduce significativamente el crecimiento de la película, cuando la concentración de $\mathrm{TBAPF}_{6}$ es menor que ca. $0.03 \mathrm{M}$ las películas directamente no se forman.

Esta dependencia con la concentración parece estar justificada en la necesidad de compensar la carga durante el crecimiento potenciodinámico de la película. De hecho, la película requiere el ingreso de cationes (probablemente protones) durante el proceso de electroreducción y su liberación en la oxidación, con el fin de compensar la carga generada por las reacciones electroquímicas confinadas en la película. Este comportamiento electroquímico es semejante al crecimiento potenciodinámico de las películas de los análogos del Azul de Prusia (Itaya, K. et al 1986, Kulesza, P. J. et al., 1999, Tacconi, N. R., Lezna, R. O. et al., 2003), así como también de los polímeros conductores, por ejemplo, polianilina y polipirrol (Hernandez, R. , 1984, Feldberg, S. W., 1984, Sadki, S. et al., 2000, Sayyah, S. M. et al., 2003, Hong, S. Y. et al., 2005, Marmisollé, W. A. et al., 2008 ) . 


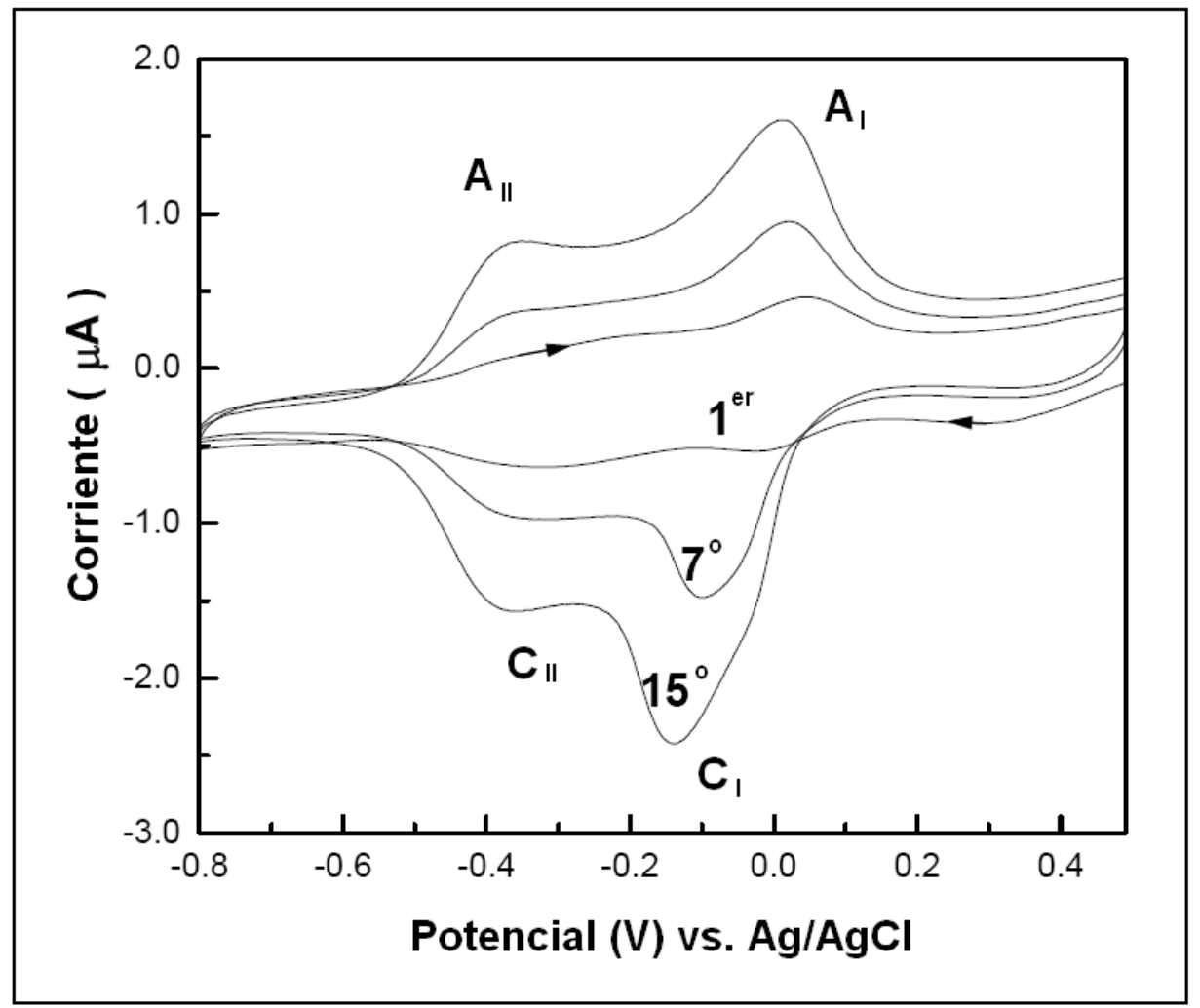

Figura 3. Voltamperogramas seleccionados $\left(1^{\mathrm{er}}, 7^{\circ}, 1^{\circ}\right)$ para $\mathrm{Zn}$-tatpp, registrados durante ciclos repetitivos a $100 \mathrm{mV} / \mathrm{s}$, en $0.15 \mathrm{M} \mathrm{TBAPF}_{6}$ utilizando $\mathrm{MeCN}: \mathrm{H}_{2} \mathrm{O}$ 90:10 como solvente. 


\subsection{Espectro de absorción para $3^{2+}$ y de las especies relacionadas formadas químicamente.}

Para identificar las especies formadas electroquímicamente en $3^{2+}$, se realizaron reducciones y protonaciones químicas estequiométricas, in situ. Estas titulaciones se realizaron en $\mathrm{MeCN}$ seco para obtener las especies reducidas no protonadas, utilizándose TFA para la protonación estequiométrica.

La figura 4 muestra los espectros de absorción tanto de las especies no protonadas, $\left[(\text { bpy })_{2} R u\left(\operatorname{tatpp}{ }^{\bullet-}\right)\right]^{+}\left(3^{\bullet+}\right),\left[(b p y){ }_{2} R u\left(\right.\right.$ tatpp $\left.\left.^{2-}\right)\right]\left(3^{0}\right)$ como de las especies protonadas $\left[(\text { bpy })_{2} \mathrm{Ru}\left(\mathrm{Htatpp}^{-}\right)\right]^{+}\left(\mathrm{H}^{+}\right)$y $\left[(\text {bpy })_{2} \mathrm{Ru}\left(\mathrm{H}_{2} \text { tatpp }\right)\right]^{2+}\left(\mathrm{H}_{2} 3^{2+}\right)$ obtenidas a partir de la valoración de $3^{2+}$ con $\mathrm{Co}(\mathrm{Cp})_{2}$ y TFA respectivamente. Co $(C p)_{2}$ es un agente reductor fuerte $\left(\mathrm{E}_{0}=-1.0 \mathrm{~V}\right.$ vs $\mathrm{Ag} / \mathrm{AgCl}$ en $\mathrm{MeCN}$ ) (Gubin, S. P. et al., 1971) y por lo tanto es termodinámicamente capaz de generar especies como $3^{\bullet+}$ y $3^{0}$ (Tacconi, N. R., Lezna, R. O. et al., 2008a). Los iones cobaltocinio generados en la titulación redox, no se observan en los espectros de absorción en el visible ni en el IR cercano (350-1100 nm) (Koelle, U. et al., 1988), por lo tanto no afectan los espectros obtenidos.

El espectro del complejo $3^{2+}$ (figura 4, línea continua) incluye la contribución del ligando tatpp a 330 y $445 \mathrm{~nm}$ (Tacconi, N. R., Lezna, R. O. et al., $2005)$ y se superpone con el del cromóforo $\left[\mathrm{Ru}(\mathrm{bpy})_{2}\right]^{2+}$ que absorbe también a $445 \mathrm{~nm}$. Los radicales $3^{\bullet+}$, formados por la adición de un equivalente de $\mathrm{Co}(\mathrm{Cp})_{2}$, muestran dos nuevas absorciones en el rojo, a 855 nm (débil) y $960 \mathrm{~nm}$ (fuerte), además de una banda a $400 \mathrm{~nm}$, así como la desaparición parcial de la absorción 
a $330 \mathrm{~nm}$. La especie doblemente reducida $3^{0}$, que se obtiene después de la adición de un segundo equivalente de $\mathrm{Co}(\mathrm{Cp})_{2}$, muestra una fuerte banda en 692 nm con un hombro en $642 \mathrm{~nm}$ (ver figura 4) y una desaparición parcial de la absorción a $400 \mathrm{~nm}$. La especie, doblemente reducida - protonada, $\mathrm{H3}^{+}$, formada con TFA, se caracteriza por bandas a $640 \mathrm{~nm}$ y $708 \mathrm{~nm}$ además de un hombro pequeño, pero perceptible, en ca. $605 \mathrm{~nm}$, mientras que el intermediario, doblemente reducido - doblemente protonado, $\mathrm{H}_{2} 3^{2+}$, muestra una banda ancha de absorción con un máximo en 565 nm (figura 4). 


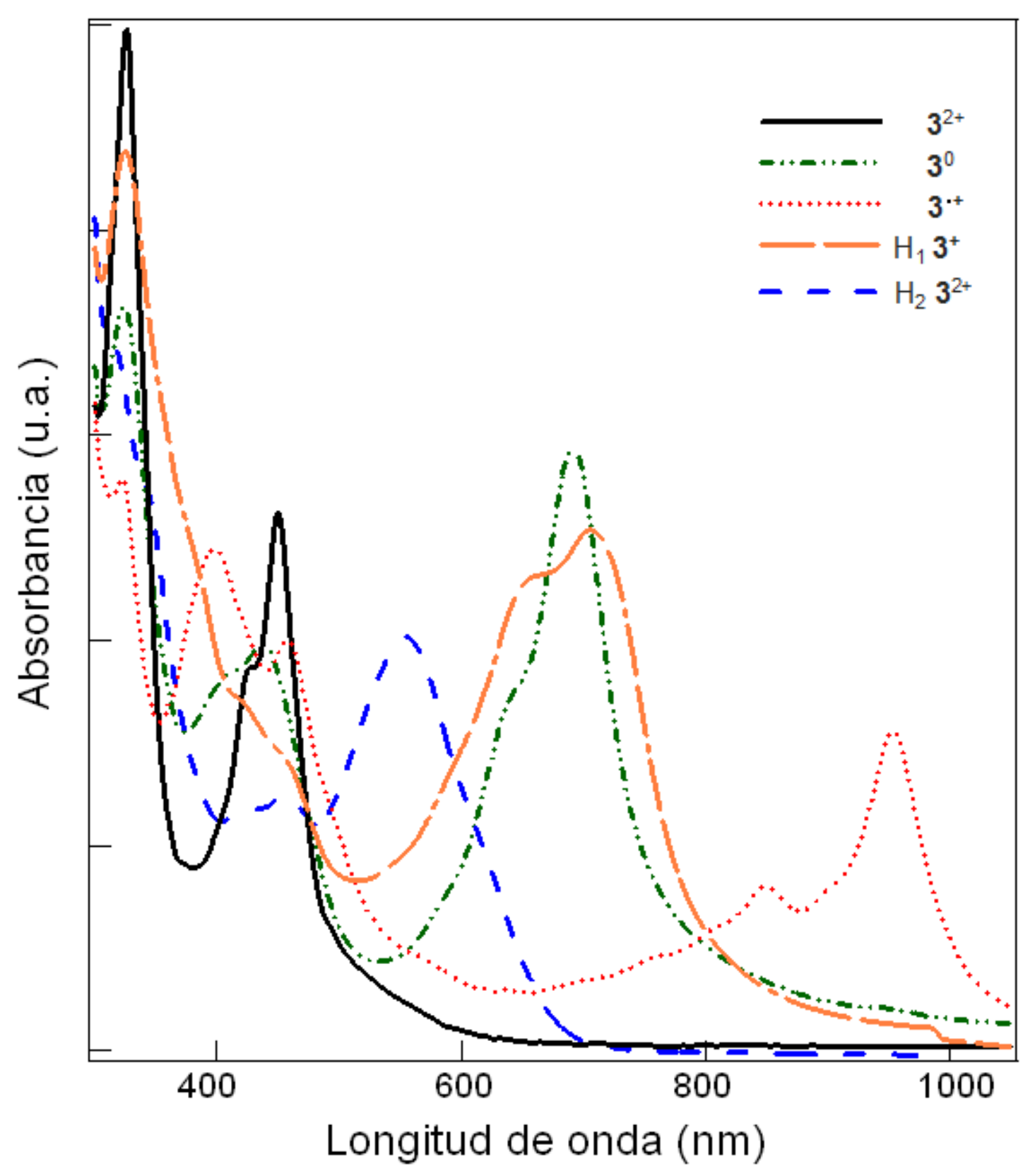

Figura 4. Espectro electrónico (MeCN) de $3^{2+}$ (línea negra) y el espectro resultante después de la adición de 2 equivalentes de $\mathrm{Co}(C p)_{2}$, que forma las especies $3^{\text {0+ }}$ (línea roja punteada ) y $3^{0}$ (línea verde punteada). Espectro de $3^{2+}$ después de la adición de 2 equivalentes de $\mathrm{Co}(\mathrm{Cp})_{2}$ y 1 equivalente de TFA , $\mathrm{H3}^{+}$, (línea naranja punteada ), 2 equivalentes de $\mathrm{Co}(C p)_{2}$ y 2 equivalentes de TFA , $\mathrm{H}_{2} 3^{2+}$, (línea azul punteada) 


\subsection{Espectroelectroquímica del complejo $\left[(\text { bpy })_{2} R u(\operatorname{tatpp})\right]^{2+}\left(3^{+2}\right)$ y del aducto $\mathrm{Zn}$-tatpp}

Los datos espectroelectroquímicos de $\mathbf{3}^{2+}$ y $\mathrm{Zn}$-tatpp se muestran en las figuras 5 y 6 respectivamente. La figura 5 muestra la evolución espectral luego de aplicar a la celda un escalón doble de potencial de 0.0 a -1.2 V (ida y vuelta) como también diferencias de espectros de absorción, $\Delta \mathrm{A}$, usando como referencia la absorbancia al potencial inicial. La perturbación utilizada se incluye en el recuadro de la figura $5 \mathrm{a}$. El espectro resultante contiene bandas que apuntan hacia arriba en el caso de aparición de nuevas especies en solución, mientras que lo contrario es válido para las bandas que crecen hacia abajo. El cuadro superior (figura 5a) corresponde al pulso de potencial negativo (reducción), y el cuadro inferior (figura 5b) se obtuvo durante el pulso positivo posterior, (oxidación); en cada cuadro, se indica con flechas la evolución temporal de los picos espectrales correspondientes. La similitud de los perfiles espectrales en las figuras $5 \mathrm{a}$ (reducción) y $5 b$ (oxidación) es una clara evidencia de la alta reversibilidad electroquímica del complejo $3^{2+}$.

Como se observa en la figura 5a, la absorción intensa en 330 y $455 \mathrm{~nm}$ (bandas apuntando hacia abajo) desaparece durante el proceso de electroreducción, mientras que dos nuevas bandas (hacia arriba) a 855 y $960 \mathrm{~nm}$ comienzan a crecer. Estas dos señales se transforman en una banda ancha con absorción en $565 \mathrm{~nm}$ con un hombro en $712 \mathrm{~nm}$. La respuesta espectroelectroquímica a 855 y $960 \mathrm{~nm}$ es consistente con la obtenida por reducción de $3^{2+}$ a $3^{*+}$ con $\mathrm{Co}(\mathrm{Cp})_{2}$ (ver figura 4) y confirma que los dos procesos (electroquímico y de reducción química) forman el mismo producto. Del mismo 
modo, la posterior formación de especies electroquímicas alcanza un máximo de absorción en 565 y $712 \mathrm{~nm}$ (figura 5a), esto es coherente con los espectros obtenidos por reducción con $\mathrm{Co}(\mathrm{Cp})_{2}$ y protonación con TFA (figura 4), mostrando

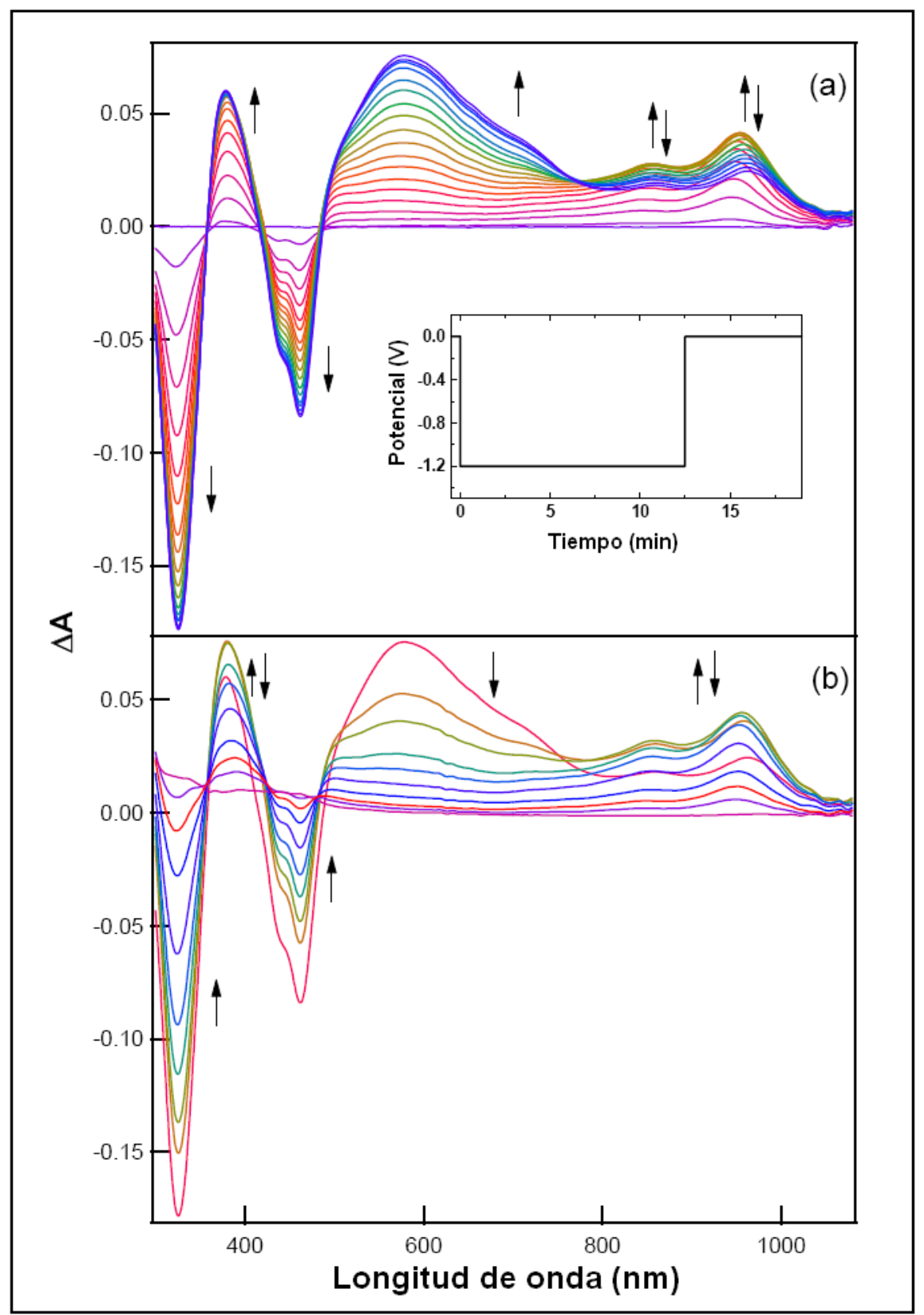

Figura 5. Diferencias de espectros de absorción, $\Delta A$, durante la electroreducción y posterior electrooxidación de $3^{2+}(50 \mu \mathrm{M})$ en solución acuosa a pH 10.5 utilizando un escalón doble de potencial de $0.0 \mathrm{Va}-1.2 \mathrm{~V}$. (a) y el regreso a $0.0 \mathrm{~V}$ (b). En el recuadro de (a) se muestra la perturbación utilizada. 
que las especies doblemente reducidas son formadas electroquímicamente en

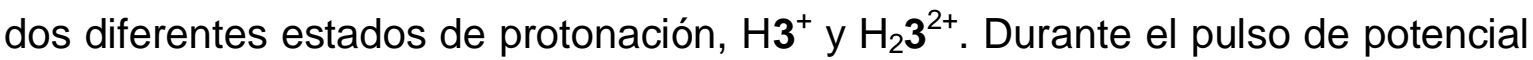
de $-1.2 \mathrm{~V}$ a $0.0 \mathrm{~V}$, se observa que los intermediarios $\mathrm{H}^{+}$y $_{2} 3^{2+}$ (712 y $565 \mathrm{~nm}$, respectivamente) se transforman en $\mathbf{3}^{\circ+}$ (con absorción en $855 \mathrm{~nm}$ y $960 \mathrm{~nm}$ ), que, a su vez, también desaparece llevando a $\Delta \mathrm{A} \rightarrow 0$ en todo el intervalo espectral. Es decir, el sistema regresa a la especie inicial $\mathbf{3}^{2+}$, cuyo espectro se tomó como referencia (figura $5 b$ ).

En la figura 7 se muestran los perfiles de absorbancia (concentración) de las especies $3^{\circ+}$ (855 nm), $\mathrm{H3}^{+}(712 \mathrm{~nm})$ y $\mathrm{H}_{2} 3^{2+}$ (565 nm) en función del tiempo (en $-1.2 \mathrm{~V}$ y en $0.0 \mathrm{~V}$ ), a las longitudes de onda correspondientes obtenidas a partir de los datos de la figura 5. En primer lugar, se observa el crecimiento con el tiempo de la especie $3^{\circ+}$ a $-1.2 \mathrm{~V}$, la que pasa por un máximo y luego decae debido a su transformación en $\mathrm{H}^{+} \mathrm{y} \mathrm{H}_{2} 3^{2+}$. La disminución de la concentración de $3^{\circ+}$ está claramente acompañada por el aumento constante de $\mathrm{H}^{+}$y $\mathrm{H}_{2} 3^{2+}$. Cuando el potencial regresa a $0.0 \mathrm{~V}$, se produce la regeneración de $3^{\circ+}$ y la disminución de las concentraciones de $\mathrm{H}^{+}{ }^{+} \mathrm{y} \mathrm{H}_{2} 3^{2+}$. Finalmente, en un tiempo relativamente corto (ca. $6 \mathrm{~min}$ ), el radical $3^{\circ+}$ se oxida para formar la especie inicial $3^{2+}$. A pesar de que la cinética para llegar a la condición de estado estacionario es diferente para los dos potenciales $(-1.2$ y $0.0 \mathrm{~V})$, los intermediarios redox involucrados reaparecen y desaparecen de tal manera que la especie $3^{2+}$ termina totalmente regenerada, por consiguiente la diferencia $A_{-1.2} \vee-A_{0.0} \vee$ retorna prácticamente a cero.

Para el aducto Zn-tatpp, la figura 6 muestra las diferencias de espectros de absorción, $\triangle \mathrm{A}$, registrados luego de dos escalones consecutivos de potencial que cubren los procesos de electroreducción $\left(\mathrm{C}_{\mathrm{I}}\right)$ y $\left(\mathrm{C}_{\|}\right)$(ver figura $2 \mathrm{~b}$ ). El potencial se pulsa a partir del valor de circuito abierto a $-0.15 \mathrm{~V}$ inicialmente (figura 6a) y luego 
a $-0.8 \mathrm{~V}$ (figura 6b). En el recuadro de la figura $6 \mathrm{a}$ se incluye el diagrama de la perturbación de potencial. En el escalón a $-0.15 \mathrm{~V}$ (figura 6 ), se forma una especie que absorbe a $855 \mathrm{~nm}$ y $960 \mathrm{~nm}$ que corresponde al radical Zn-tatpp ${ }^{\circ}$, sin embargo, esta especie es detectada solamente en cantidades menores (significativamente inferior a la de $3^{\bullet+}$, ver figura $5 a$ ). La especie dominante absorbe a 570, 638 y $692 \mathrm{~nm}$ y parece ser una mezcla del aducto doblemente reducido $\mathrm{Zn}$-(tatpp $\left.{ }^{2-}\right)$ en sus formas simple $\mathrm{Zn}$ - (Htatpp-) y doblemente protonado $\mathrm{Zn}-\left(\mathrm{H}_{2}\right.$ tatpp). La aparición de estructura vibracional, bien resuelta en la banda, sugiere que $\mathrm{Zn}$-(Htatpp-) es la especie dominante presente, ya que esta estructura se observa típicamente en el ligando protonado - doblemente reducido tatpp de $3^{2+}$ y $2^{4+}$. (Tacconi, N. R., Lezna, R. O. et al., 2005, 2008a, 2008b).

Estas bandas son significativamente mucho más agudas que las obtenidas en los espectros correspondientes a la electroreducción del complejo $3^{2+}$ (figura $5 a)$, resultado atribuido a los efectos del solvente (tatpp no se puede solubilizar en agua para comparar este comportamiento en el mismo medio que $3^{2+}$ ). Cuando el potencial es cambiado a $-0.8 \mathrm{~V}$, es decir, a potenciales más negativos que los del proceso $\mathrm{C}_{\|}$, las absorciones a 570, 638 y $692 \mathrm{~nm}$ disminuyen durante este segundo proceso de electroreducción (figura 6b). Este comportamiento contrasta con la observación de una acumulación continua de especies doblemente reducidas durante la electroreducción de $3^{2+}$ a potenciales más negativos que $\mathrm{C}_{\| I}$ (figura $5 \mathrm{a}$ ). 


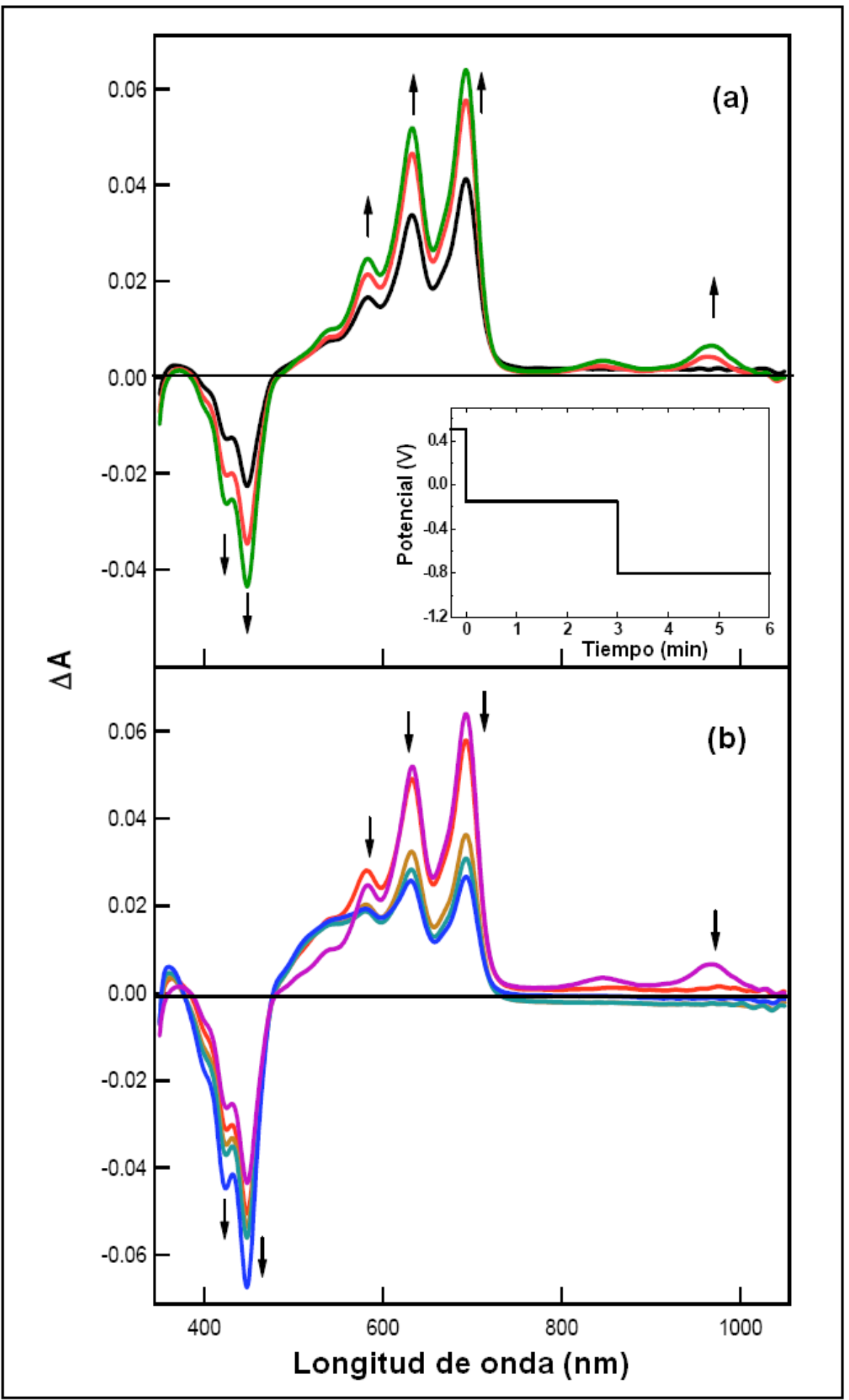

Figura 6. Diferencias de espectros de absorción, $\triangle A$, durante la electroreducción de $\mathrm{Zn}$-tatpp (60 $\mu \mathrm{M}$ ) en MeCN: $\mathrm{H}_{2} \mathrm{O}$ 90:10, en respuesta a 2 pulsos de potencial sucesivos (mostradas en el recuadro de (a)) del potencial a circuito abierto a $-0.15 \mathrm{~V}$ (a) y de $-0.15 a-0.8 \mathrm{~V}(b)$. 


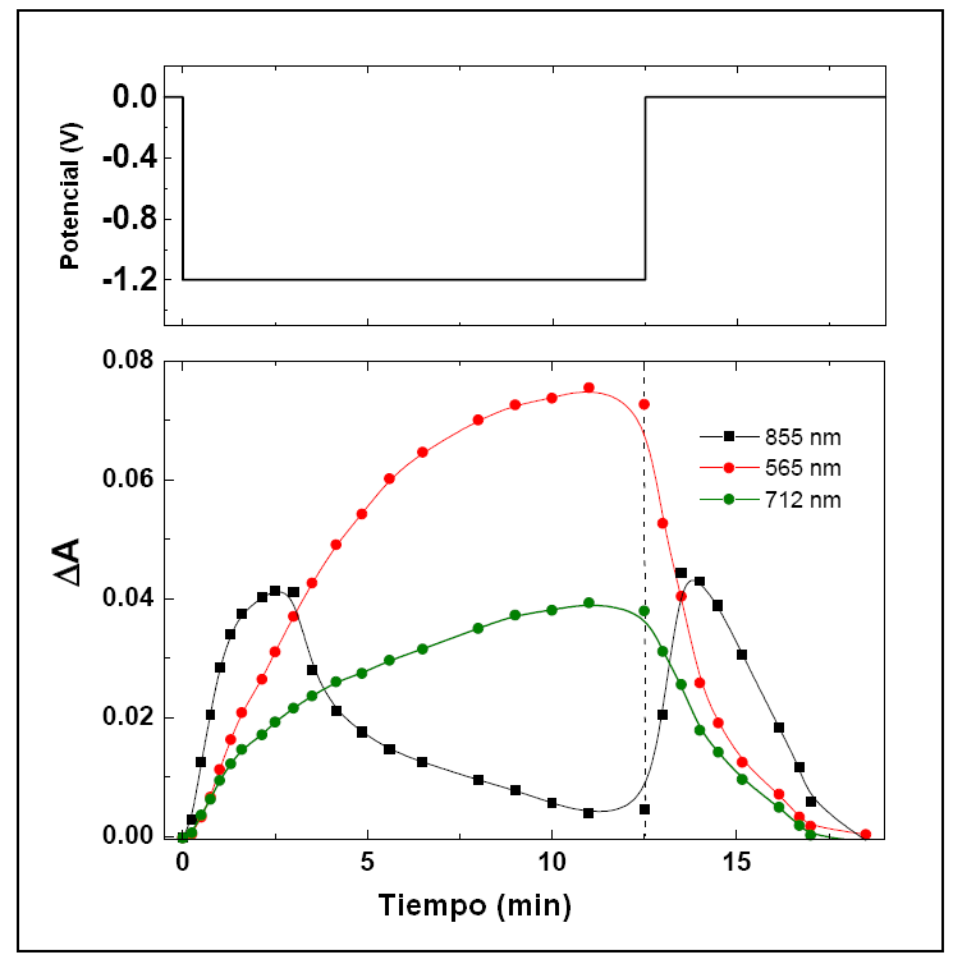

Figura 7. Perfil de $\Delta A$ vs. tiempo a longitudes de onda seleccionadas. (855 $\mathrm{nm}$ para $\mathbf{3}^{\mathbf{+}}$, $712 \mathrm{~nm}$ para $\mathrm{H}^{+}$y $565 \mathrm{~nm}$ para $\mathrm{H}_{2} 3^{2+}$ ). Datos de la figura 5 . 


\subsection{Discusión}

En el complejo $3^{2+}$, la primera especie electroreducida es el radical $\mathbf{3}^{\circ+}$, que se identifica fácilmente por las dos absorciones a longitudes de onda, 855 y 960 nm (Tacconi, N. R., Lezna, R. O. et al., 2008b). Estas bandas son claramente identificadas en la figura 5 , en su formación (fig. 5a) y desaparición (fig. 5b). La separación de ca. $1300 \mathrm{~cm}^{-1}$ sugiere que es probable que la estructura vibracional sea $v_{0-1}$ y $v_{0-0}$, respectivamente, en la misma transición electrónica. Los picos de baja energía en el espectro electrónico son comunes para los aniones radicales aromáticos (Fox, M. A.,1978), lo que permite observar la fracción tatpp del complejo $\left[(\text { bpy })_{2} R u\left(\text { tatpp }{ }^{*}\right)\right]^{+}\left(3^{*+}\right)$. La formación de radicales $3^{*+}$ produce la desaparición de las dos absorciones centradas en el ligando ( $L C$ ), $L C_{1}$ a $330 \mathrm{~nm}$ y $\mathrm{LC}_{0}$ con una absorción estructurada en $450 \mathrm{~nm}$ con un hombro significativo en $424 \mathrm{~nm}$ (fig. 4 y 5). Estas bandas se encuentran convenientemente interpretadas con una descripción MO localizada del ligando tatpp. $\mathrm{LC}_{0}$ es asignado a una transición $\pi-\pi_{0}{ }^{*}$ (ligando tatpp HOMO-LUMO), mientras que LC ${ }_{1}$ es una transición HOMO a LUMO +1 de tatpp $\left(\pi-\pi_{1}^{*}\right)$, como se comprueba por la presencia de la banda de absorción estructurada $\mathrm{LC}_{0}$ en la espectroelectroquímica del aducto $\mathrm{Zn}$ tatpp (ver figura 6). Es importante destacar que, en el complejo $3^{2+}$, la transición $\mathrm{LC}_{0}$ de tatpp se superpone con una transición más ancha MLCT $\mathrm{Ru}(\mathrm{d} \pi) \rightarrow$ ligando $\left(\pi^{\star}\right)$ centrada en ca. $450 \mathrm{~nm}$, típica de complejos Ru (II)-phen y Ru (II)bpy. El resultado neto es una banda intensa que alcanza un máximo en $450 \mathrm{~nm}$ con un coeficiente de extinción molar de $43.300 \mathrm{M}^{-1} \mathrm{~cm}^{-1}$ (Tacconi, N. R., Lezna, R. O. et al., 2005). 
El siguiente proceso de electroreducción para el radical $3^{\circ+}$ está asociado con la aparición de una banda ancha con un máximo en 565 nm y un hombro en ca. $710 \mathrm{~nm}$ (figura 5a). Esta banda está compuesta por las contribuciones de las especies de la doble reducción, protonada $\mathrm{H}_{1} 3^{+}$y doblemente protonada $\mathrm{H}_{2} 3^{2+}$ (ver figura 4). Es importante destacar que la aparición de estas bandas de absorción para las especies simple y doblemente reducidas coinciden con la desaparición parcial o total de las transiciones tatpp LC (absorción fuerte a 330 $\mathrm{nm}$ y $450 \mathrm{~nm}$ ), ya que la electroreducción se localiza en el ligando tatpp. A potenciales más negativos al proceso de electroreducción $\mathrm{C}_{\|}$, no hay desaparición de las bandas características de las especies $\mathrm{H}_{1} 3^{+}$ni $\mathrm{H}_{2} 3^{2+}$.

En el aducto Zn-tatpp, se detectan cantidades mínimas del radical Zn(tatpp*) a través de las bandas a $855 \mathrm{~nm}$ y $960 \mathrm{~nm}$, en potenciales que corresponden al proceso $C_{\text {I }}$ (figura 6 ). Este radical está presente en una cantidad relativamente menor que en el complejo $3^{2+}$ electroreducido. De hecho, las principales especies electroreducidas en el potencial $C_{l}$, se observan a través de la magnitud de las bandas correspondientes $(570 \mathrm{~nm}, 638 \mathrm{~nm}$ y $692 \mathrm{~nm}$ ) relacionadas con las especies doblemente-reducidas y protonadas, $\mathrm{Zn}$-(Htatpp $)$ y $\mathrm{Zn}$-( $\mathrm{H}_{2}$ tatpp). La fuente de protones es el agua en la mezcla. La especie doblemente reducida de $\mathrm{Zn}$-tatpp también comparte similitudes espectrales con $3^{0}, \mathrm{H}_{1} 3^{+}$y $\mathrm{H}_{2} 3^{2+}$. Cuando se protona $3^{0}$ para dar $\mathrm{H}^{+}{ }^{+}$y $\mathrm{H}_{2} 3^{2+}$ se observa un corrimiento de la absorción de la banda LC hacia el azul (de $692 \mathrm{~nm}$ a $565 \mathrm{~nm}$ ) (figura 4), como era de esperar de la estabilización de los pares libres de nitrógeno centrados en tatpp (Turro, N. J.,1991). Al cambiar el potencial a valores más negativos para incluir el proceso de electroreducción $\mathrm{C}_{\| 1}$, las bandas de $\mathrm{Zn}$ (Htatpp) y $\mathrm{Zn}$ ( $\mathrm{H}_{2}$ tatpp) desaparecen (figura 6b), a diferencia del caso cuando tatpp está coordinado, por ejemplo, en el complejo $\mathbf{3}^{+2}$. 


\subsection{Mecanismo de electroreducción}

En el complejo de $3^{2+}$, el pico $\mathrm{C}_{\text {l }}$ es en general un proceso de $2 \mathrm{e}^{-} / 2 \mathrm{H}^{+}$ (reacción 1), $\mathrm{H}_{2} 3^{2+}$ es siempre el producto dominante detectado espectroelectroquímicamente. Las reacciones de la 2 a la 5 muestran una serie de pasos elementales mediante los cuales puede ocurrir la reacción 1. Los términos $\mathbf{E}$ y $\mathbf{C}$ dados a las reacciones de la 2 a la 5 , son usados para describir un mecanismo secuencial que involucra reacciones tanto electroquímicas como químicas (Saveant, J-M., 2006). Las reacciones que implican la transferencia de electrones (ET) se indican con una $\mathbf{E}, \mathrm{y}$ de las reacciones químicas de primer orden o pseudo-de primer orden (por ejemplo, protonación) se indican con una C. El proceso general $\mathrm{C}_{\mid}$se describe en la reacción 1 :

$$
\begin{aligned}
& 3^{2+}+2 \mathrm{e}^{-}+2 \mathrm{H}^{+} \rightleftarrows \mathrm{H}_{2} 3^{2+} \\
& 3^{2+}+\mathrm{e}^{-} \rightleftarrows 3^{0^{+}} \mathrm{E} \\
& 3^{\circ+}+\mathrm{e}^{-} \rightleftarrows 3^{0} \mathrm{E} \\
& 3^{0}+\mathrm{H}^{+} \rightleftarrows \mathrm{H}^{+} \quad \mathrm{C} \\
& \mathrm{H}^{+}+\mathrm{H}^{+} \rightleftarrows \mathrm{H}_{2} 3^{2+} \mathrm{C}
\end{aligned}
$$

A pesar de que el proceso $C_{\mid}$no se resuelve en picos separados, el proceso consta de dos etapas de reducción de un electrón según la detección espectroelectroquímica, produciendo inicialmente $3^{\circ+}$ (reacción 2) y posteriormente una mezcla de $\mathrm{H}^{+}{ }^{+} \mathrm{y} \mathrm{H}_{2} 3^{2+}$. El radical monoreducido $3^{\circ+}$ no está protonado y su reducción requiere en primer lugar una ET seguida de transferencia de protones (PT), como se indica en las reacciones 3 y $4 . \mathrm{H}_{2} 3^{2+}$ se forma probablemente a través de la protonación de $\mathrm{H}^{+}$(reacción 5). Como $\mathrm{H}_{2} 3^{2+}$ 
es el producto dominante, este mecanismo coincide en gran medida con un proceso total de $2 \mathrm{e}^{-} / 2 \mathrm{H}^{+}$.

A los potenciales del proceso $\mathrm{C}_{\|}$, se observa una acumulación continua del producto doblemente reducido que absorbe a $712 \mathrm{~nm}\left(\mathrm{H}^{+}\right)$y $565 \mathrm{~nm}\left(\mathrm{H}_{2} 3^{2+}\right)$ (figuras 5 a y 7). Sin embargo, la desaparición de las bandas ópticas de $\mathrm{H}^{+}$y $\mathrm{H}_{2} 3^{2+}$ se ha observado claramente mediante espectroelectroquímica reportada en (Tacconi, N. R., Lezna, R. O. et al., 2008b), donde se confirma un mecanismo de $2 \mathrm{e}^{-/} \mathrm{2H}^{+}$(reacción 6) que conduce a la desaparición de la especie doblemente reducida en la capa de difusión, de la siguiente manera:

$$
\mathrm{H}_{2} 3^{2+}+2 \mathrm{e}^{-}+2 \mathrm{H}^{+} \rightleftarrows \mathrm{H}_{4} 3^{2+}
$$

No se espera que el producto de la reacción $6, \mathrm{H}_{4} 3^{2+}$, muestre bandas de absorción en el visible debido al llenado completo de sus orbitales moleculares LUMO y LUMO +1 en las formas protonadas del ligando tatpp ${ }^{4-}$. Esto también se observa en el complejo dinuclear $\mathrm{H}_{4} 2^{4+}$, que muestra una desaparición de todas las bandas visibles LC (Konduri, $R$. et al., 2004). En las medidas espectroelectroquímicas (figuras 5 y 7) se ha observado una acumulación constante de $\mathrm{H}_{2} 3^{2+}$ conduciendo a la formación de $\mathrm{H}_{4} 3^{2+}$ (reacción 6); sólo en presencia de una reacción química se consume rápidamente esta especie y se regenera $\mathrm{H}_{2} 3^{2+}$. Esta reacción (reacción 7) podría ser la comproporcionación (COMP) de $\mathrm{H}_{4} 3^{2+}$ con $3^{2+}$, que ocurriría en solución (por ejemplo, reacción 7).

$$
\mathrm{H}_{4} 3^{2+}+3^{2+} \rightleftarrows 2 \mathrm{H}_{2} 3^{2+} \quad \text { COMP }
$$

Esta suposición se ha confirmado a través del seguimiento espectroscópico hecho a $\mathrm{H}_{2} 3^{2+}$, una vez que la aplicación de potencial se ha interrumpido (circuito abierto), mediante la detección de la especie $\mathrm{H}_{2} 3^{2+}$ generada químicamente a través de la transferencia homogénea de electrones (reacción 7) entre $\mathrm{H}_{4} 3^{2+}$ y $3^{2+}$ en solución. La figura 8 muestra el crecimiento de la especie $\mathrm{H}_{2} 3^{2+}$ cuando el potencial se desconecta inmediatamente después de un barrido lineal de 
potencial entre $0.0 \mathrm{~V}$ hasta $-1.2 \mathrm{~V}$. La especie $\mathrm{H}_{2} 3^{2+}$ se detecta ópticamente a 565 $\mathrm{nm}$ durante su formación electroquímica (hasta $-1.2 \mathrm{~V}$, figura 8a) y también el incremento de su concentración después de que el circuito se ha abierto, reacción 7 (figura 8b).

La formación química de la especie $\mathrm{H}_{2} 3^{2+}$, se observa durante ca. $180 \mathrm{~s}$ hasta que difunde fuera de la trayectoria del haz. Esta medida proporciona una explicación de la acumulación continua de especies $\mathrm{H}_{2} 3^{2+}$ en los datos espectroelectroquímicos obtenidos a potenciales negativos del pico $\mathrm{C}_{\| I}$ (figura 5a).

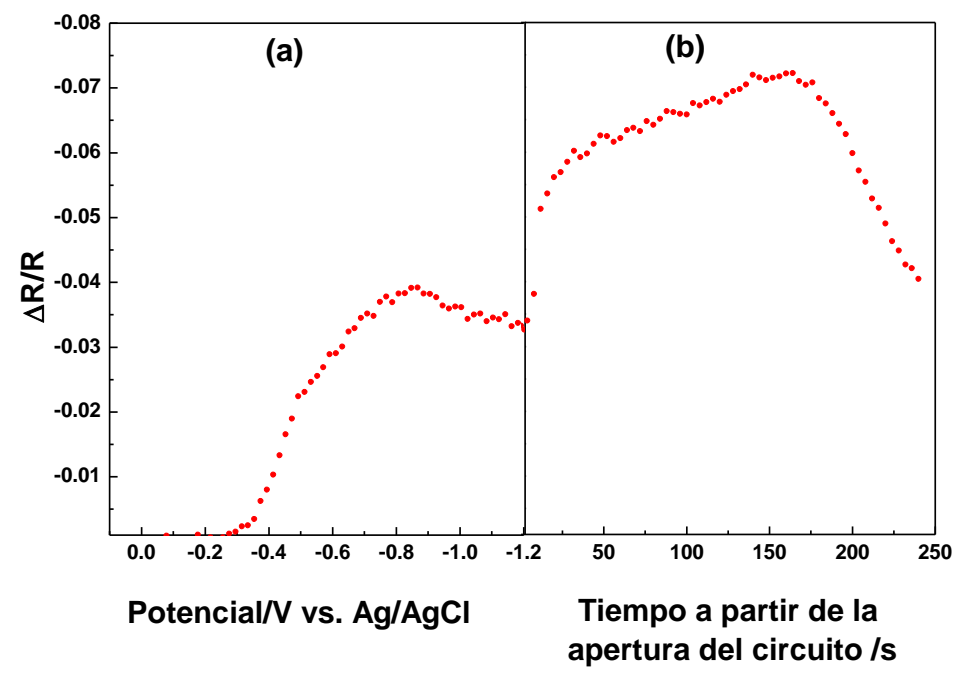

Figura 8. Reflectancia integral normalizada $\Delta R / R$, (a) monitoreando la presencia de $\mathrm{H}_{2} 3^{2+}$ (565 nm) a pH 10.5, durante un barrido de potencial de $0.0 \mathrm{~V} \mathrm{a}-1.2 \mathrm{~V}$ (que abarca los procesos $C_{1}$ y $\left.C_{l l}\right)(b)$ generación química de $\mathrm{H}_{2} 3^{2+}$ después de la apertura de circuito. En (a) y (b) los espectros fueron tomados con un OMA a intervalos de $4 \mathrm{~s} \equiv 20 \mathrm{mV}$.

En Zn-tatpp, la electroreducción $\mathrm{C}_{\text {| }}$ también se asocia a un proceso de 2 electrones (figura. 2b), donde predominan las formas $\mathrm{Zn}$ - (Htatpp') y en menor medida $\mathrm{Zn}-\left(\mathrm{H}_{2}\right.$ tatpp). Durante este proceso se observa una pequeña cantidad de 
Zn-(tatpp"), confirmando su papel como intermediario en el proceso global de las reacciones 8 a la 11 (omitiendo Zn para mayor claridad).

\begin{tabular}{|c|c|c|c|c|}
\hline $\begin{array}{l}\text { tatpp + } \\
\text { tatpp }{ }^{-}+\end{array}$ & $\begin{array}{l}\mathrm{e}^{-} \\
\mathrm{H}^{+}\end{array}$ & $\begin{array}{l}\rightleftarrows \\
\rightleftarrows\end{array}$ & $\begin{array}{l}\text { tatpp } \\
\text { Htatpp• }\end{array}$ & $\begin{array}{l}E \\
C\end{array}$ \\
\hline 2 Htatpp & $\rightleftarrows$ & tatpp + & $\mathrm{H}_{2}$ tatpp & DISP \\
\hline $\mathrm{H}_{2}$ tatpp & $\rightleftarrows$ & Htatpp & $\mathrm{D}^{-}+\mathrm{H}^{+}$ & C \\
\hline
\end{tabular}

La formación de Zn-(Htatpp $)^{*}$ es precedida por una primera electroreducción que produce $\mathrm{Zn}$-(tatpp*) (detectado en pequeñas cantidades), en equilibrio con $\mathrm{Zn}$-(Htatpp). Esta última especie es inestable y sufre rápidamente desproporcionación (reacción 10, de desproporcionación DISP) para dar Zn( $\mathrm{H}_{2}$ tatpp) y $\mathrm{Zn}$-tatpp (figura 6). La desprotonación de $\mathrm{Zn}$ - ( $\mathrm{H}_{2}$ tatpp) conduce a que Zn-(Htatpp-) sea el producto principal.

Este comportamiento contrasta con la coordinación de tatpp en el complejo $\mathbf{3}^{2+}$, donde el radical relacionado, $\mathbf{3}^{\mathbf{*}}$, se observa en cantidades relativamente mucho mayores, lo que indica menor protonación y por lo tanto, menor desproporcionación. Así, la reducción durante el proceso $C_{\mid}$para $3^{2+}$, transcurre principalmente a través de dos secuencias de un electrón cada una seguido por protonación. En $\mathrm{Zn}$-tatpp, el proceso $\mathrm{C}_{\text {। }}$ es también un proceso global de dos electrones que involucra una reducción a un radical anión, una protonación y una desproporcionación.

En el intervalo de potenciales que cubren el proceso $\mathrm{C}_{\| 1}$, tiene lugar la reducción de 2 electrones tanto de $\mathrm{H}_{2} 3^{2+}$ (reacción 6) como de $\mathrm{Zn}$ - (Htatpp) (reacción 12) a la especie cuádruplemente reducida del tatpp que no posee transiciones ópticas visibles. 


$$
\text { Zn- }\left(\text { Htatpp }^{-}\right)+2 \mathrm{e}^{-} \rightleftarrows \mathrm{Zn}-\left(\mathrm{Htatpp}^{3-}\right) \quad \mathrm{E}
$$

No se espera que los productos de las reacciones de 6 y 12 o formas protonadas de las mismas, muestren bandas de absorción en el visible debido a la ocupación de los orbitales moleculares, vide supra, del ligando tatpp cuádruplemente reducido. Esto se observó claramente en $\mathrm{Zn}$ - $\left(\mathrm{Htatpp}^{3-}\right)$, pero no con $\mathrm{H}_{4} 3^{4+}$. Existen dos razones para este comportamiento. En Zn-(Htatpp $\left.{ }^{3-}\right)$, el complejo parece formar una película sobre la superficie del electrodo de ITO que limita su difusión hacia la solución. En cambio en el caso de $\mathrm{H}_{4} 3^{4+}$, el complejo se encuentra en la interfase y la difusión hacia el seno de la solución conduce a la reacción 7 de comproporcionación, que fue seguida con claridad a circuito abierto (figura 8). 


\subsection{Conclusiones}

El complejo $3^{2+}$ muestra cuatro reducciones reversibles en el ligando central tatpp. Las reacciones redox ocurren mayormente como dos procesos de $2 \mathrm{e}-/ 2 \mathrm{H}^{+}$manifestadas en los picos voltamperométricos $\mathrm{C}_{\mid}$y $\mathrm{C}_{\|}$respectivamente.

La onda voltamperométrica $\mathrm{C}_{1}$ implica la formación de $\mathrm{H}^{+}$y $\mathrm{H}_{2} 3^{2+}$ pasando por el intermediario $\mathbf{3}^{\bullet+}$. Las especies fueron monitoreadas a través de medidas espectroelectroquímicas. $\mathrm{La}$ onda voltamperométrica $\mathrm{C}_{\|}$refleja una doble reducción y una doble protonación que conduce a la formación del producto $\mathrm{H}_{4} 3^{2+}$; esta especie sufre una rápida comproporcionación con $3^{2+}$ (presente en el seno de la solución) que conduce a su desaparición. Las mediciones ópticas realizadas a circuito abierto (figura 8 ), aportan una evidencia clara del continuo crecimiento de $\mathrm{H}_{2} 3^{2+}$ a través de la reacción de comproporcionación (reacción 7).

El aducto Zn-tatpp sufre reducciones múltiples acompañadas de protonaciones de un modo bastante similar a las del complejo $3^{2+}$. Sin embargo, muestra tres diferencias esenciales con respecto a $3^{+2}$ :

i) Las especies Zn-tatpp forman una película durante los procesos de electroreducción, mientras que el complejo $3^{2+}$ no lo hace.

ii) Se forman cantidades insignificantes de las primeras especies electroreducidas (radical tatpp`*) a lo largo de la onda voltamperométrica 
$\mathrm{C}_{\mathrm{l}}$, mientras que se detectó una cantidad mucho mayor del radical relacionado, $3^{*}$, durante la electroreducción del complejo $3^{2+}$. Tanto, tatpp" como $3^{\circ-}$ fueron seguidos por las mismas bandas en el infrarrojo cercano.

iii) En el proceso $C_{\|}$de electroreducción del complejo $3^{2+}$, ocurre una reacción rápida de comproporcionación; en contraste con la ausencia de esta reacción en el aducto Zn-tatpp. La libertad de movimiento de las moléculas de $3^{2+}$ en solución probablemente sea responsable de la reacción rápida de comproporcionación; mientras que en el segundo caso, las especies se encuentran confinadas sobre la superficie del electrodo y por tanto se inhibe su capacidad de movimiento evitando el encuentro con especies similares en solución.

Es importante destacar que este trabajo muestra la información adquirida mediante el uso de espectroelectroquímica en el seguimiento de las especies electroreducidas de tatpp ya sea como aducto $\mathrm{Zn}$-tatpp o como complejo $3^{2+}$. La comprensión de los detalles mecanísticos asociados con la configuración molecular proporciona un marco útil para describir y eventualmente modificar la capacidad de aceptación de múltiples electrones del ligando tatpp. 


\subsection{Referencias}

Alstrum-Acevedo, J. H.; Brennaman, M. K.; Meyer, T. J. Inorg. Chem. 2005, $44,6802$.

Armaroli, N. y Balzani, V. Angew. Chem. Int. Ed. 2007, 46, 52.

Balzani, V.; Ceroni, P.; Maestri, M.; Saudan, C. Y Vicinelli, V. Top. Curr. Chem. 2003, 228, 159.

Borgström, M.; Shaikh, N.; Johansson, O.; Anderlund, M. F.; Styring, S.; Akermark, B.; Magnuson, A. y Hammarström L. J. Am. Chem. Soc. 2005, 127, 17504.

Cukier, R. I. y Nocera, D. G. Ann. Rev. Phys. Chem. 1998, 49, 337.

Chang, C. C.; Pfennig, B. y Bocarsly, A. B. Coord. Chem. Rev. 2000, 2008, 33.

Dempsey, J. L.; Esswein, A. J.; Manke, D. R.; Rosenthal, J.; Soper, J. D. y Nocera, D. G. Inorg. Chem. 2005, 44, 6879.

Eisenberg, R. y Nocera, D. G. Inorg. Chem. 2005, 44, 6779.

Elvington, M. y Brewer, K. J. Inorg. Chem. 2006, 45, 5242.

Esswein, A. J.; Veige, A. S. y Nocera, D. G. J. Am. Chem. Soc. 2005, 127, 16641. 
Feldberg, S. W. J. Am. Chem. Soc. 1984, 106, 4671 - 4614

Fox, M. A. Chem. Rev. 1978, 78, 253.

Gubin, S. P.; Smirnova, S. A. y Denisovich, L. I. J. Organomet. Chem. 1971, 30, 257.

Heyduk, A. F. y Nocera, D. G. Science 2001, 293, 1639.

Hernandez, R. ; Diaz, A.F. ; Waltman, R y Bargon, J. The Journal of Physical Chemistry, 1984, 88 (15) 3333 - 3337.

Hong, S. Y.; Jung, Y. M.; Bin Kim, S. y Park, S. M. J. Phys. Chem. B 2005, 109,3855 .

Huynh, M. H. y Meyer, T. J. Chem. Rev. 2007, 107, 5004.

Itaya, K.; Uchida, I. y Neff, V. D. Acc. Chem. Res. 1986, 19, 162.

Janaratne, T. K.; Yadav, A.; Ongeri, F. y MacDonnell, F. M. Inorg. Chem. 2007, 46, 3420 .

Kirmaier, K. y Holten, D. The Photosynthetic Bacterial Reaction Center Structure and Dynamics; Plenum: New York, 1988.

Koelle, U.; Infelata, P. P. y Grätzel, M. Inorg. Chem. 1988, 27, 879.

Konduri, R.; Ye, H.; MacDonnell, F. M.; Serroni, S.; Campagna, S. y Rajeshwar, K. Angew. Chem. Int. Ed. 2002, 41, 3185.

Konduri, R.; de Tacconi, N. R.; Rajeshwar, K. y MacDonnell, F. M. J. Am. Chem. Soc. 2004, 126, 11621. 
Kulesza, P. J.; Malik, M. A.; Skorek, J.; Smolinska, A.; Miecznikowski, K.; Zamponi, S.; Berrettoni, M.; Giorgetti, M. y Marassi, R. J. Electroanal. Chem. 1999, 146, 3757.

Marmisollé, W. A.; Posadas,D. y Florit, M. I. J. Phys. Chem. B. 2008, 112, $10800-10805$.

Molnar, S. M.; Nallas, G.; Bridgewater, J. S. y Brewer, K. J. J. Am. Chem. Soc. 1994, 116, 5206.

Pfennig, B. W.; Mordas, C. J.; McCloskey, A.; Lockard, J. V.; Salmon, P. M.; Cohen, J. L.; Watson, D. F. y Bocarsly, A. B. Inorg. Chem. 2002, 41, 4389.

Rosenthal, J.; Bachman, J.; Dempsey, J. L.; Esswein, A. J.; Gray, T. G.; Hodgkiss, J. M.; Manke, D. R.; Luckett, T. D.; Pistorio, B. J.; Veige, A. S. y Nocera, D. G. Coord. Chem. Rev. 2005, 249, 1316.

Sadki, S.; Schottland, P.; Brodie, N y Sabouraud, G. Chem. Soc. Rev., 2000, 29, 283.

Saveant, J-M. Elements of Molecular and Biomolecular Electrochemistry - An Electrochemical Approach to Electron Transfer Chemistry; Wiley-Interscience: Hoboken, NJ, 2006.

Sayyah, S. M.; Abd El-Rehim, S. S. y El-Deeb, M. M. J. Appl. Polymer Sci. 2003, 90, 1783.

Sun, L.; Hammarstrom, L.; Akermark, B. y Styring, S. Chem. Soc. Rev. 2001, 30,36 . 
Tacconi, N. R.; Rajeshwar, K. y Lezna, R. O. Chem. Mater. 2003, 15, 3046; y referencias incluidas.

Tacconi, N. R.; Lezna, R. O.; Konduri, R.; Ongeri, F.; Rajeshwar, K. y MacDonnell, F. M. Chem. Eur. J. 2005, 11, 4327.

Tacconi, N. R.; Chitakunye, R.; Macdonnell, F. M. y Lezna, R. O. J. Phys. Chem. A, 2008 a, 112, 497.

Tacconi, N. R.; Lezna, R. O.; Chitakunye, R. y Macdonnell, F. M. Inorg. Chem 2008 b, 47, 8847.

Tommos, C. y Babcock, G. T. Acc. Chem. Res. 1998, 31, 18.

Turro, N. J. Modern Molecular Photochemistry; University Science Books: Sausalito, CA, 1991. 


\section{Espectroelectroquímica de} radicales aniones generados en la electroreducción de -

$$
\begin{gathered}
{\left[\operatorname{Re}^{\prime}(\mathrm{CO})_{3}(5-N i t r o-1,10-\right.} \\
\text { fenantrolina })]^{+} y-
\end{gathered}
$$

$\left[\operatorname{Re}^{\prime}(\mathrm{CO})_{3}(3,4,7,8\right.$-tetrametil-1,10fenantrolina) $]^{+}$en polímeros de poli- 4-vinilpiridina 
Espectroelectroquímica de complejos TF-5N, TF-TM,PV-5N, PV-TM 2 Capítulo 5. 

3

Capítulo 5. Espectroelectroquímica de radicales aniones generados en la electroreducción de $-\left[\operatorname{Re}^{\prime}(\mathrm{CO})_{3}(5-N i t r o-1,10-\text { fenantrolina })\right]^{+}$y $\quad$ $\left[\operatorname{Re}^{\prime}(\mathrm{CO})_{3}(3,4,7,8 \text {-tetrametil-1,10-fenantrolina) }]^{+}\right.$en polímeros de poli- 4vinilpiridina.

5.1 Medidas electroquímicas.

5.2 Espectroelectroquímica de los complejos TF-5N, TF-TM, PV-5N, PV-TM.

5.3 Discusión.

5.4 Conclusiones.

5.5 Referencias. 
Polímeros inorgánicos sólidos y en solución han sido objeto de numerosos estudios en relación a sus reacciones térmicas y fotoquímicas. El interés de realizar medidas electroquímicas y espectroelectroquímicas con este tipo de sistemas, especialmente con complejos de la forma $X \mathrm{Re}^{\prime}(\mathrm{CO})_{3} \mathrm{~L}$, es debido a su potencial uso, entre otros, en catálisis (e.g., algunos complejos monoméricos de la forma $\mathrm{XRe}(\mathrm{CO})_{3} \mathrm{~L}$, actúan como fotocatalizadores y/o electrocatalizadores para la reducción de $\mathrm{CO}_{2}$ a productos de interés), (Hawecker, J. et al., 1983, Kalyanasundaram, K. et al., 1993, Costamagna, J. et al., 1996, Vogler, A. et al., 2000) en dispositivos ópticos (Stufkens, D.J. et al., 1998, Wolcan, E. et al., 2000), y como sensores luminiscentes (Yoon, D.I. et al., 1992, Sacksteder, L. et al., 1993, Yam, V.W-W. et al., 1995); estos complejos, también pueden ser utilizados en áreas tales como estudios de transferencia de electrones (Fox, M.A. et al., 1988) y conversión de energía solar (Kalyanasundaram, K.,1982). Los complejos de metales de transición luminiscentes de $R e$ (I) y $R u$ (II) con ligandos polipiridil han sido reconocidos como potenciales candidatos a ser materiales útiles para medir pH (Higgins, B. et al., 2005).

Las propiedades de los polímeros $\left\{(\mathrm{vpy})_{2}-\mathrm{vpy}\left[\operatorname{Re}^{\prime}(\mathrm{CO})_{3}(\text { phen }]^{+}\right\}_{n \sim 200} \quad\right.$ y $\left\{(\text { vpy })_{2}-\mathrm{vpy}\left[\operatorname{Re}^{\prime}(\mathrm{CO})_{3}(\text { bpy })\right]^{+}\right\}_{n \sim 200}$ (figura 1 y 2 ) en fase orgánica fueron investigados en trabajos previos (Wolcan, E. et al., 2000,2003,2005, Bracco, L.L.B. et al.,2008, 2009, 2010, 2011). 
(a)

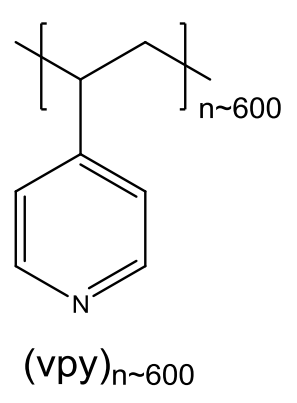

(b)

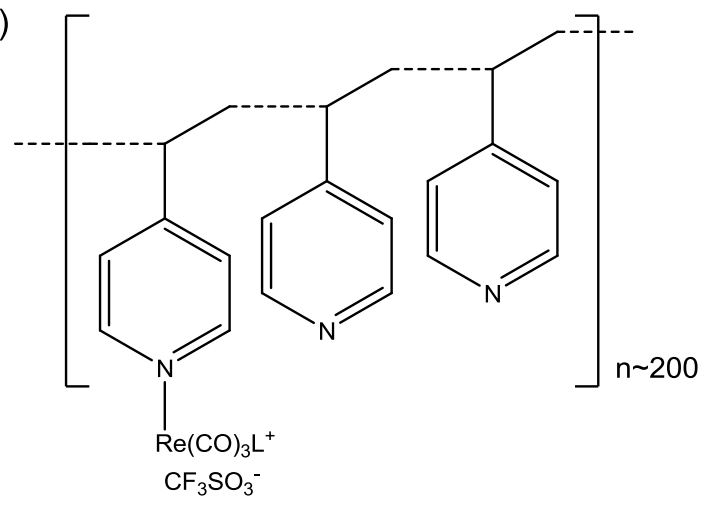

Figura 1. Fórmula estructural para (a) poly-4-(vinilpiridina), (b) polímeros derivados de la poli-4-(vinilpiridina).

El tema de este capítulo corresponde al estudio químico y electroquímico de radicales formados en la electroreducción de los complejos $\left[\mathrm{CF}_{3} \mathrm{SO}_{3}\right.$ $\operatorname{Re}^{\prime}(\mathrm{CO})_{3}\left(\mathrm{NO}_{2}\right.$-phen)],(TF-5N), [ $\mathrm{CF}_{3} \mathrm{SO}_{3} \mathrm{Re}^{\prime}(\mathrm{CO})_{3}($ tmphen)], (TF-TM), y de los polímeros $\mathrm{CF}_{3} \mathrm{SO}_{3}\left[\operatorname{Re}(\mathrm{CO})_{3}\left(\mathrm{NO}_{2}\right.\right.$-phen)-P4VPy] $(\boldsymbol{P V}-5 \mathrm{~N})$ y $\mathrm{CF}_{3} \mathrm{SO}_{3}\left[\operatorname{Re}(\mathrm{CO})_{3}\right.$ (tmphen)-P4VPy] (PV-TM), los polímeros contienen los complejos de $\operatorname{Re}(\mathrm{I})$ coordinados a los grupos piridínicos de la poli-4-vinilpiridina $\left(\mathrm{NO}_{2}\right.$-phen: 5-Nitro1,10-fenantrolina, tmphen: 3,4,7,8-tetrametil-1,10-fenantrolina, P4VPy: Poli-4vinilpiridina). La estructura de cada complejo se puede visualizar en las figuras 2 y 3 . 


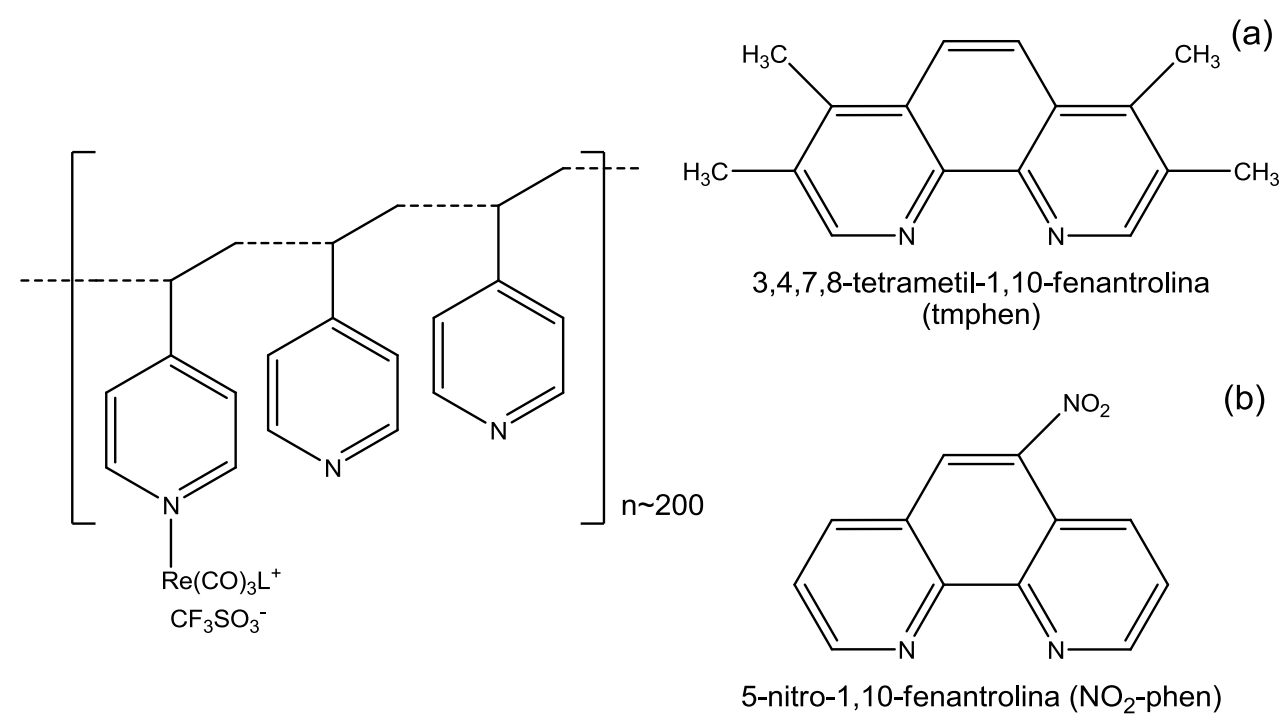

Figura 2. Estructura del polímero: Poli-4- vinilpiridina [P4VPy], cuando L es: (a) tmphen, se forma el complejo PV-TM. Cuando $L$ es (b) $\mathrm{NO}_{2}$-phen, se forma el complejo PV-5N.
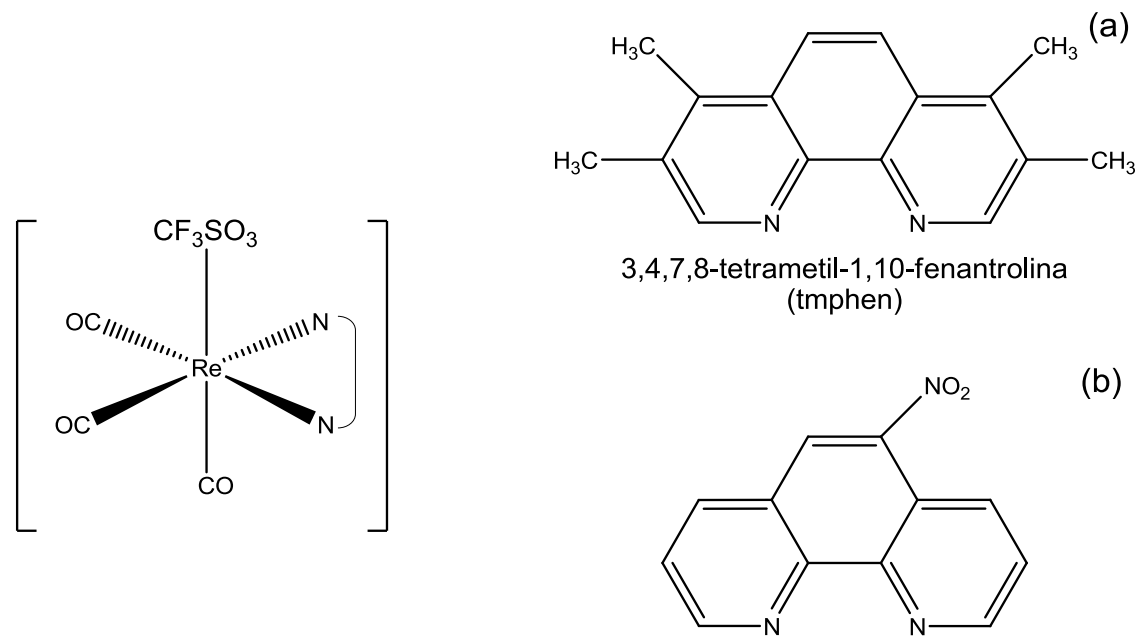

(b)

5-nitro-1,10-fenantrolina $\left(\mathrm{NO}_{2}\right.$-phen)

Figura 3. Complejos de Re (I), coordinados a: (a) tmphen, formando el complejo TF-TM. (b) $\mathrm{NO}_{2}$-phen, formando TF-5N. 


\subsection{Medidas electroquímicas}

Se llevaron a cabo medidas de voltamperometría de alterna con cada uno de los complejos de estudio (TF-TM, TF-5N, PV-TM y PV-5M), siguiendo el método descripto en el capítulo 2 (Materiales y Métodos). Se utilizó MeCN como solvente, $\mathrm{TBAPF}_{6}$ como electrolito soporte y $\mathrm{Ag} / \mathrm{AgCl}$ como electrodo de referencia, en el intervalo de potencial comprendido entre $0.0 \mathrm{~V}$ y $-2.0 \mathrm{~V}$.

En la figura 4 ( $a$ y b), se observan los ACVs para los complejos TF-TM y TF-5N respectivamente. El voltamperograma de TF-TM (figura 4a), presenta 3 picos de reducción en $-1.366 \mathrm{~V},-1.570 \mathrm{~V}$ y $1.766 \mathrm{~V}$, correspondientes a procesos de 1 e cada uno; estas reducciones se asignan al ligando tmphen del complejo. El voltamperograma de la figura 4b, correspondiente a la reducción de TF-5N, muestra 4 picos reversibles de $1 \mathrm{e}^{-}$en $-0.527 \mathrm{~V},-1.087 \mathrm{~V},-1.514 \mathrm{~V},-1.716 \mathrm{~V}$. La primera reducción se localiza en el grupo $\mathrm{NO}_{2}$ mientras que las tres restantes corresponden a la reducción de la parte phen del ligando $\mathrm{NO}_{2}-$ phen. 
(a)

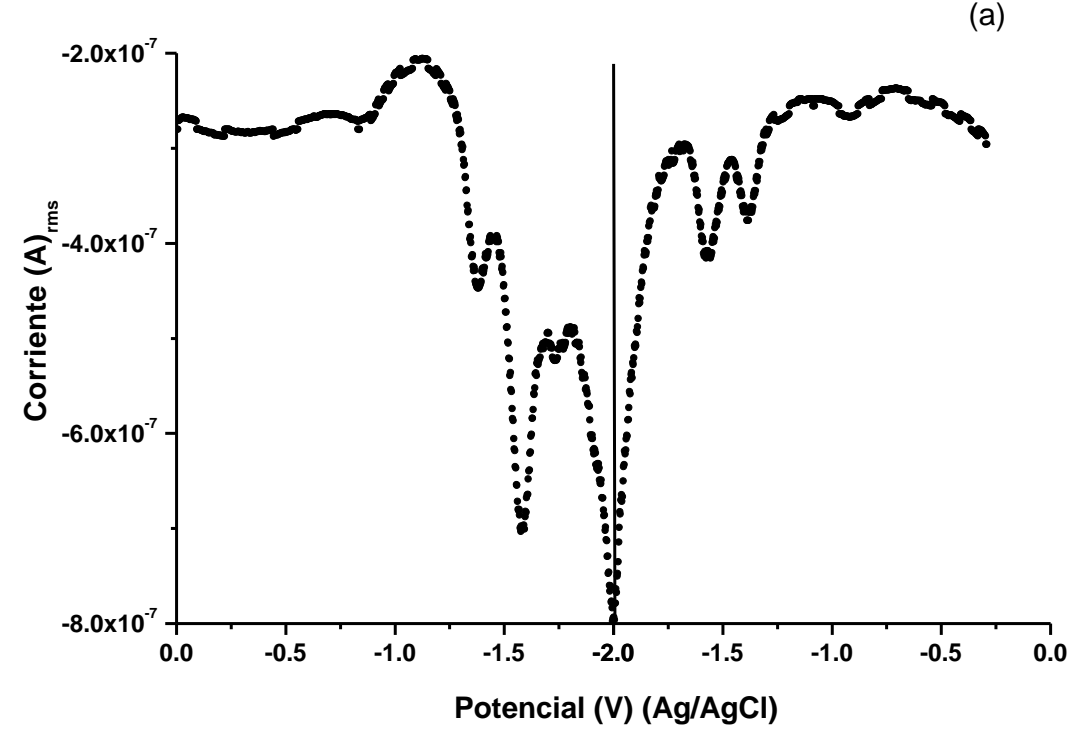

(b)

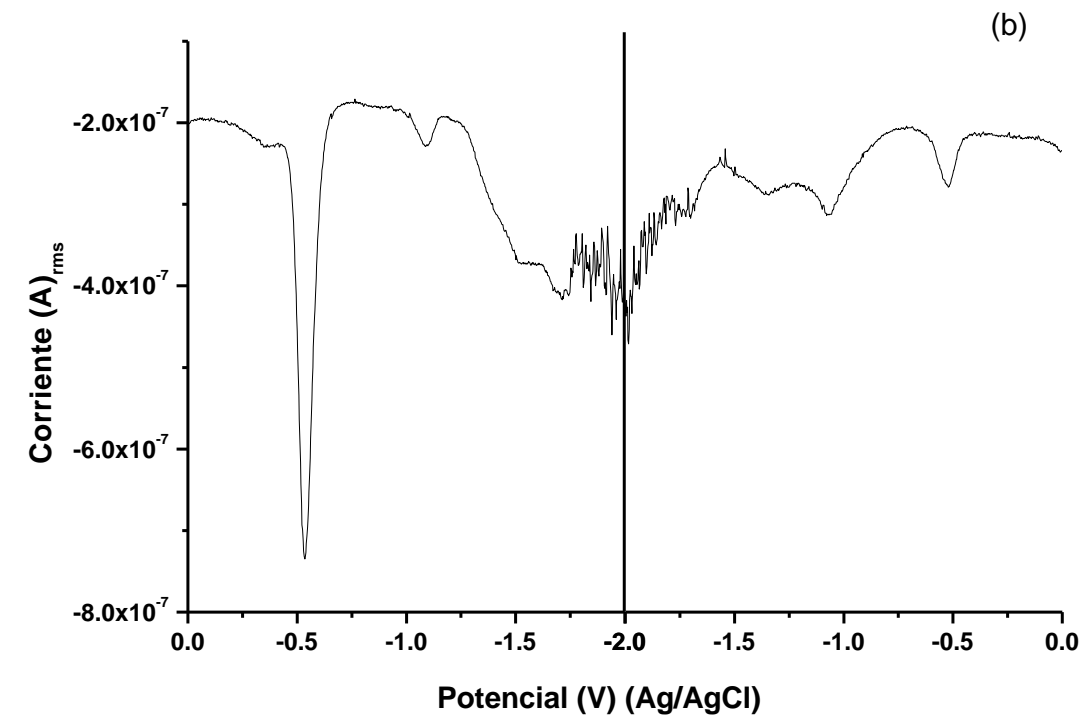

Figura 4. ACV de los complejos, (a) TF-TM, (b) TF-5N, en MeCN. Electrolito soporte TBAPF 6 . 

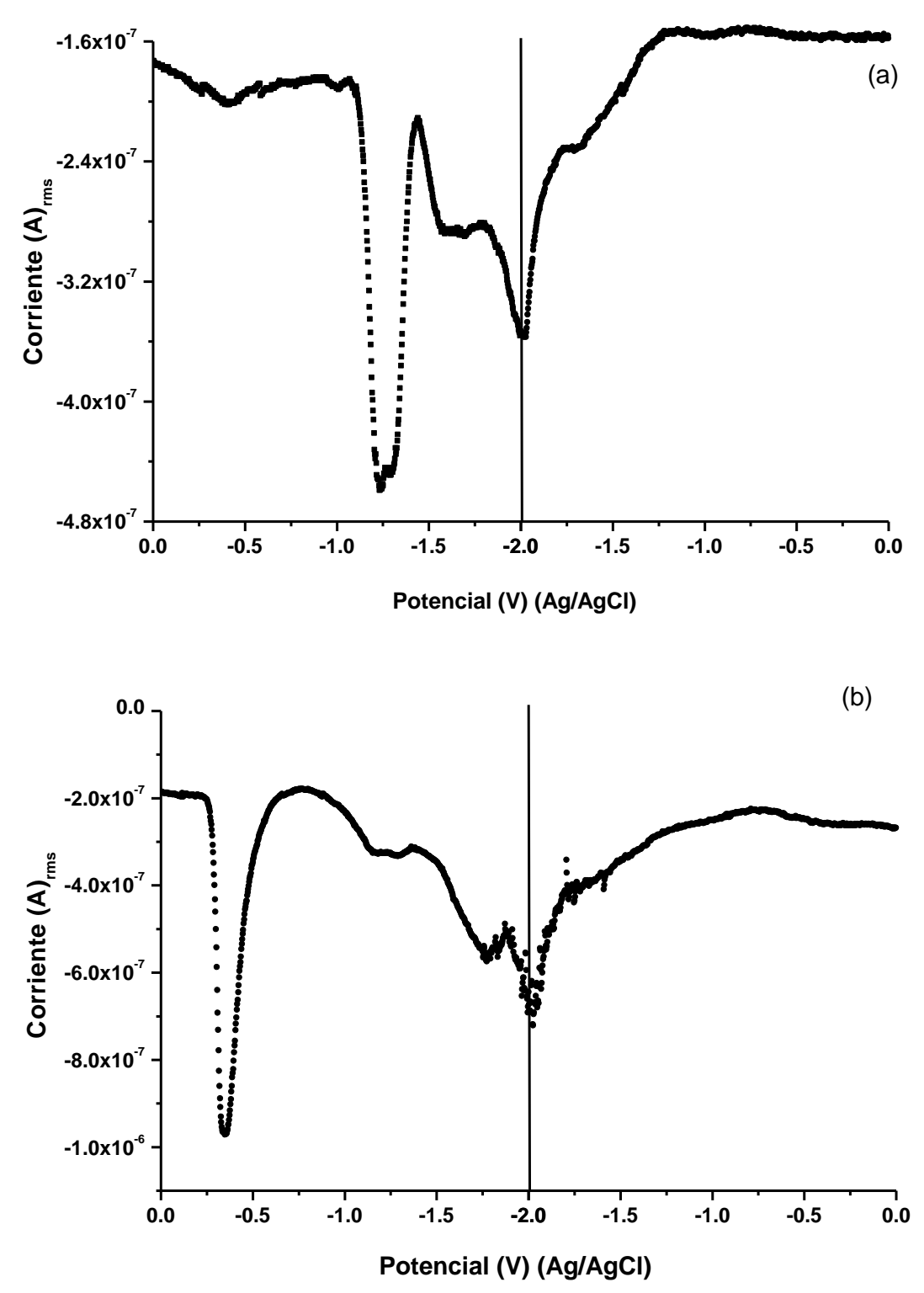

Figura 5. ACV de los complejos, (a) $P V-T M$, (b) $P V-5 N$, en MeCN. Electrolito soporte TBAPF $_{6}$.

Los voltamperogramas correspondientes a los complejos $\boldsymbol{P V}-\mathbf{T M}$ y $\boldsymbol{P V}-\mathbf{5 N}$ se observan en la figura 5. El complejo PV-TM, muestra tres reducciones de $1 \mathrm{e}^{-}$, irreversibles en $-1.231 \mathrm{~V},-1.339 \mathrm{~V}$ y $-1.590 \mathrm{~V}$ (figura 5a), asignadas a la reducción en el ligando tmphen del complejo; mientras que $\boldsymbol{P V}-5 \mathbf{N}$ presenta 4 
reducciones irreversibles de $1 \mathrm{e}^{-}$en $-0.349 \mathrm{~V},-1.157 \mathrm{~V},-1.296 \mathrm{~V}$ y $-1.773 \mathrm{~V}$, el primer potencial de reducción está relacionado con la parte $\mathrm{NO}_{2}$ del ligando, mientras que las tres siguientes reducciones ocurren en la porción phen del ligando (figura 5b).

Los potenciales de reducción de los complejos analizados (TF-5N, TF-TM, $\boldsymbol{P V}-\mathbf{5 N}$ y $\boldsymbol{P V}$-TM) están en concordancia con valores descriptos en la literatura para complejos tipo $\mathrm{XRe}^{\prime}(\mathrm{CO})_{3} \mathrm{~L}$ (ver tabla 1). (Luong, J.C. et al., 1978, Kalyanasundaram, K., 1986, Paolucci, F. et al., 1998, Gabrielsson, A. et al., 2005). Los potenciales presentados en la tabla 1, inicialmente se han reportado vs. electrodos diferentes a $\mathrm{Ag} / \mathrm{AgCl}$, por lo tanto, es necesario realizar su conversión para comparar resultados (tercera columna de la tabla 1) de acuerdo con Pavlishchuk et al.(Pavlishchuk, V.V. et al., 2000).

Tabla 1. Potenciales de reducción para complejos $X \operatorname{Re}^{\prime}(C O)_{3} L$.

\begin{tabular}{|c|c|c|}
\hline Complejos & $E(V)$ & $\mathrm{E}(\mathrm{V}) \mathrm{vs} . \mathrm{Ag} / \mathrm{AgCl}$ \\
\hline $\mathrm{ClRe}(\mathrm{CO})_{3}($ phen $)$ & $\begin{array}{l}-1.34 \text { vs. SCE }{ }^{a} \\
-1.27 \text { vs. SCE } \\
-1.36 \text { vs. SCE }\end{array}$ & $\begin{array}{l}-1.287 \\
-1.217 \\
-1.307\end{array}$ \\
\hline $\operatorname{Re}(\mathrm{CO})_{3}($ phen $)\left(\mathrm{CH}_{3} \mathrm{CN}\right)^{+}$ & -1.24 vs. SCE ${ }^{c}$ & -1.187 \\
\hline $\mathrm{ClRe}(\mathrm{CO})_{3}\left(\mathrm{NO}_{2}\right.$-phen $)$ & $-1.01,-1.68$ vs. $\mathrm{Fc} / \mathrm{Fc}^{+d}$ & $-0.589,-1.259$ \\
\hline $\begin{array}{l}{\left[\mathrm{CF}_{3} \mathrm{SO}_{3} \mathrm{Re}^{\prime}(\mathrm{CO})_{3}\left(\mathrm{NO}_{2-}^{-}\right.\right.} \\
\text {phen })] \mathrm{TF}-5 \mathrm{~N}\end{array}$ & $\begin{array}{l}-0.527,-1.087,-1.514 \\
-1.716 \text { vs. } \mathrm{Ag} / \mathrm{AgCl}\end{array}$ & $\begin{array}{l}-0.527,-1.087 \\
-1.514,-1.716\end{array}$ \\
\hline$\left[\mathrm{CF}_{3} \mathrm{SO}_{3} \operatorname{Re}^{\prime}(\mathrm{CO})_{3}(\mathrm{tmphen})\right]$ & $-1.366,-1.570,-1.766$ vs. & $-1.366,-1.570,-1.766$ \\
\hline
\end{tabular}




\begin{tabular}{|c|c|c|}
\hline$T F-T M$ & $\mathrm{Ag} / \mathrm{AgCl}$ & \\
\hline $\begin{array}{l}\mathrm{CF}_{3} \mathrm{SO}_{3}\left[\mathrm{Re}(\mathrm{CO})_{3}\left(\mathrm{NO}_{2^{-}}\right.\right. \\
\text {phen)-P4VPy] } \boldsymbol{P V}-5 \mathbf{N}\end{array}$ & $\begin{array}{l}-0.349,-1.157,-1.296 \\
-1.773 \text { vs. } \mathrm{Ag} / \mathrm{AgCl}\end{array}$ & $\begin{array}{l}-0.349,-1.157 \\
-1.296,-1.773\end{array}$ \\
\hline $\begin{array}{l}\mathrm{CF}_{3} \mathrm{SO}_{3}\left[\mathrm{Re}(\mathrm{CO})_{3} \text { (tmphen)- }\right. \\
\text { P4VPy] } \boldsymbol{P V}-\mathbf{T M}\end{array}$ & $\begin{array}{l}-1.231,-1.339,-1.590 \text { vs. } \\
\mathrm{Ag} / \mathrm{AgCl}\end{array}$ & $-1.231,-1.339,-1.590$ \\
\hline
\end{tabular}

a. (Luong, J.C. et al., 1978).

b. (Kalyanasundaram, K., 1986).

c. (Paolucci, F. et al., 1998)

d. (Gabrielsson, A. et al., 2005) 


\subsection{Espectroelectroquímica de los complejos $T F-5 N, T F-T M, P V-5 N, P V-$}

\section{TM.}

Con el fin de identificar las especies generadas electroquímicamente, asociadas a las ondas medidas por $\mathrm{ACV}$, se tomaron espectros in-situ a intervalos de $40 \mathrm{mV}$ con la ayuda de un OMA, utilizando una velocidad de barrido de $5 \mathrm{mV}$ $\mathrm{s}^{-1}$ en el intervalo de potencial entre 0.0 y $-2.0 \mathrm{~V}$. El primer espectro en $0.0 \mathrm{~V}$, en el cual no se detectó absorción, fue utilizado como referencia.

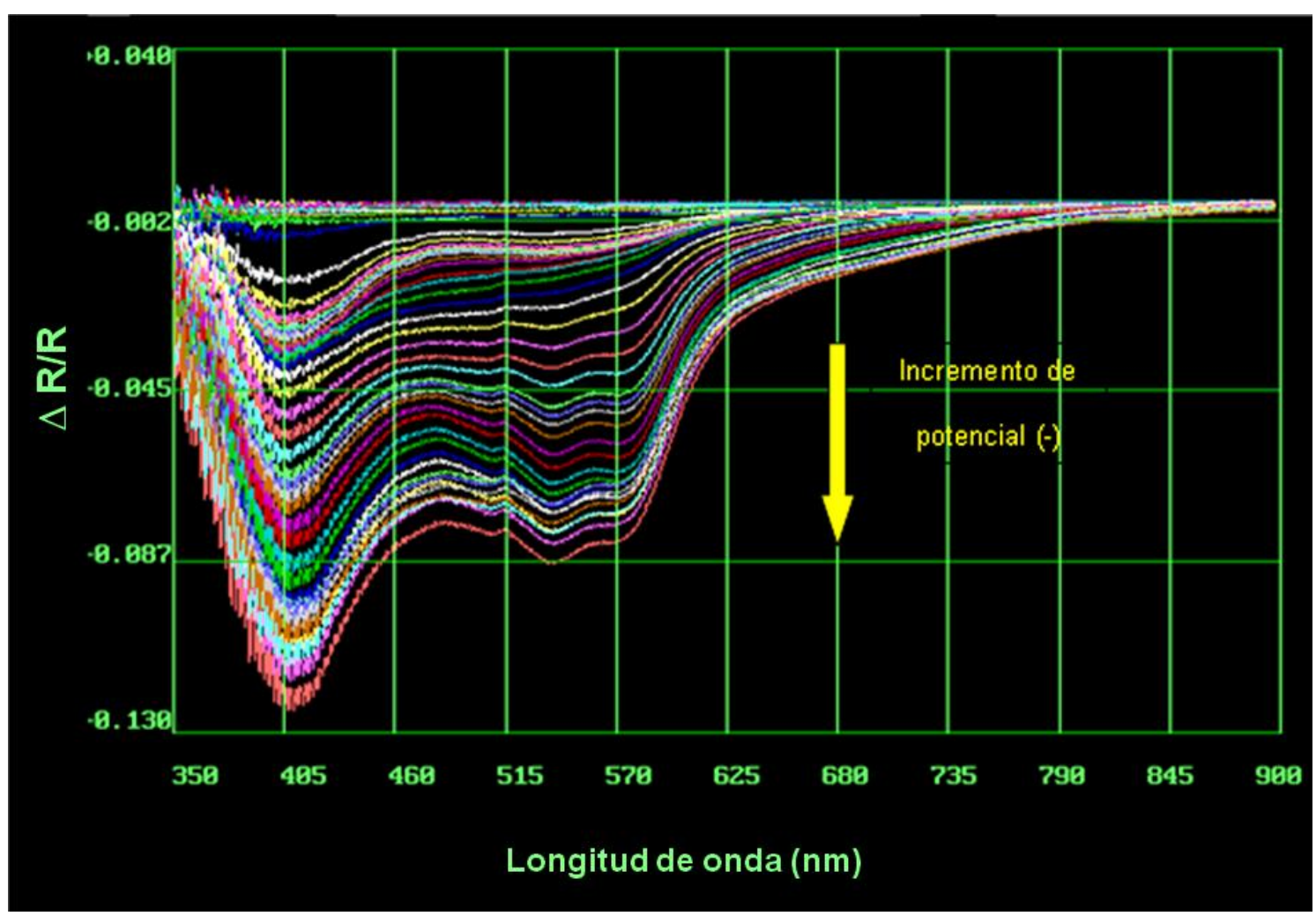

Figura 6. Respuesta espectroelectroquímica de TF-5 N, utilizando un OMA. $\mathrm{MeCN} / \mathrm{TBAPF}_{6}, 0.0-2.0 \mathrm{~V} / 5 \mathrm{mV} \mathrm{s}^{-1}$ 
En la figura 6, se muestra la respuesta medida con un OMA para el complejo TF-5N ( $\Delta \mathrm{R} / \mathrm{R} \sim \Delta \mathrm{A})$, cada espectro corresponde a un potencial determinado a lo largo del barrido entre 0.0 y $-2.0 \mathrm{~V}$. A medida que aumenta el potencial negativamente, se observa el crecimiento de una banda aguda, $\downarrow$, a 410 $\mathrm{nm}$ y una ancha a $535 \mathrm{~nm}$ que posteriormente se divide en dos componentes. Esta respuesta indica la aparición de nuevas especies producto de la reacción de reducción.

A continuación se presentan los espectros de interés para cada complejo, identificando las especies asociadas a las ondas medidas por ACV.

La figura 7a, muestra con claridad un conjunto reducido de espectros para $\left[\operatorname{Re}^{\prime}(\mathrm{CO})_{3}\left(\mathrm{NO}_{2} \text {-phen) }\left(\mathrm{CH}_{3} \mathrm{CN}\right)\right]^{+}(\mathrm{TF}-5 \mathrm{~N})\right.$, donde cada espectro es tomado a valores de potencial correspondiente a los picos de reducción descriptos en la figura $4 \mathrm{~b}$. Entre -0.5 y $-1.08 \mathrm{~V}$, se observa una banda aguda a $\lambda=410 \mathrm{~nm}$ y una ancha a $\lambda=535 \mathrm{~nm}$, indicando la reducción del grupo nitro del ligando $\mathrm{NO}_{2}$ phen, para formar el anión radical $\left[\left(\mathrm{NO}_{2}{ }^{-}\right)\right.$- phen]. A potenciales más negativos, es decir entre -1.0 y - $2.0 \mathrm{~V}$, la banda a $535 \mathrm{~nm}$ se divide en dos componentes en 543 y $570 \mathrm{~nm}$; el aumento de la banda a ca. $535 \mathrm{~nm}$ indica los procesos de reducción que tienen lugar en la porción phen del ligando $\mathrm{NO}_{2}$ - phen, formando $\left[\operatorname{Re}^{\prime}(\mathrm{CO})_{3}\left(\mathrm{NO}_{2}{ }^{\circ-}\right)-\left(\text { phen }{ }^{\circ}\right)\left(\mathrm{CH}_{3} \mathrm{CN}\right)\right]^{-}$como producto de la segunda reducción. (Wolcan, E. et al., 2000, Bracco, L.L.B. et al., 2009).

En los espectros correspondientes a $\boldsymbol{P V}-5 \boldsymbol{N}$ (figura $7 \mathrm{~b}$ ), se observan cambios similares (a TF-5N) aunque menos definidos. Las bandas observadas a $410 \mathrm{~nm}$ corresponden a la formación de $\left[\operatorname{Re}(\mathrm{CO})_{3}\left(\mathrm{NO}_{2}{ }^{--}\right)\right.$- (phen)] P4VPy y a potenciales más negativos el proceso de reducción se lleva a cabo en la parte phen del ligando, generando $\left[\operatorname{Re}^{\prime}(\mathrm{CO})_{3}\left(\mathrm{NO}_{2}{ }^{-*}\right)-\left(\text { phen }{ }^{*-}\right)\right]^{-}$P4VPy. Los potenciales 
seleccionados para generar los espectros mostrados en la figura $7 \mathrm{~b}$ provienen de los picos de reducción del ACV de la figura $5 b$.

En la figura 8a se muestran los espectros para TF-TM, a los potenciales tomados de la figura 4 a. Se observan 3 bandas, una aguda a $440 \mathrm{~nm}$ con un hombro a $420 \mathrm{~nm}$ y una banda ancha a $565 \mathrm{~nm}$ para el intervalo de potencial entre -1.3 y -1.8 V; estas bandas se atribuyen al producto de la reducción del grupo phen en el complejo, formando el radical anión (tmphen ${ }^{*}$ ). Cambios similares se observan en la reducción del polímero $\boldsymbol{P} \boldsymbol{V}-\boldsymbol{T M}$, figura 8b. Los espectros de la figura 8b, fueron seleccionados teniendo en cuenta los potenciales de reducción graficados en la figura 5a. 
(a)

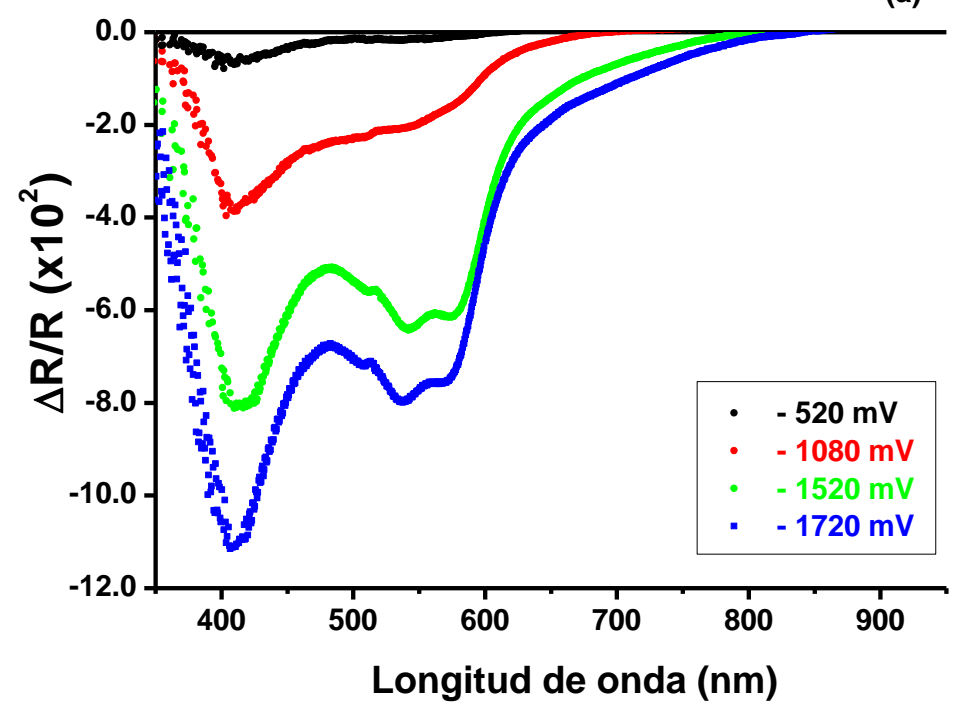

(b)

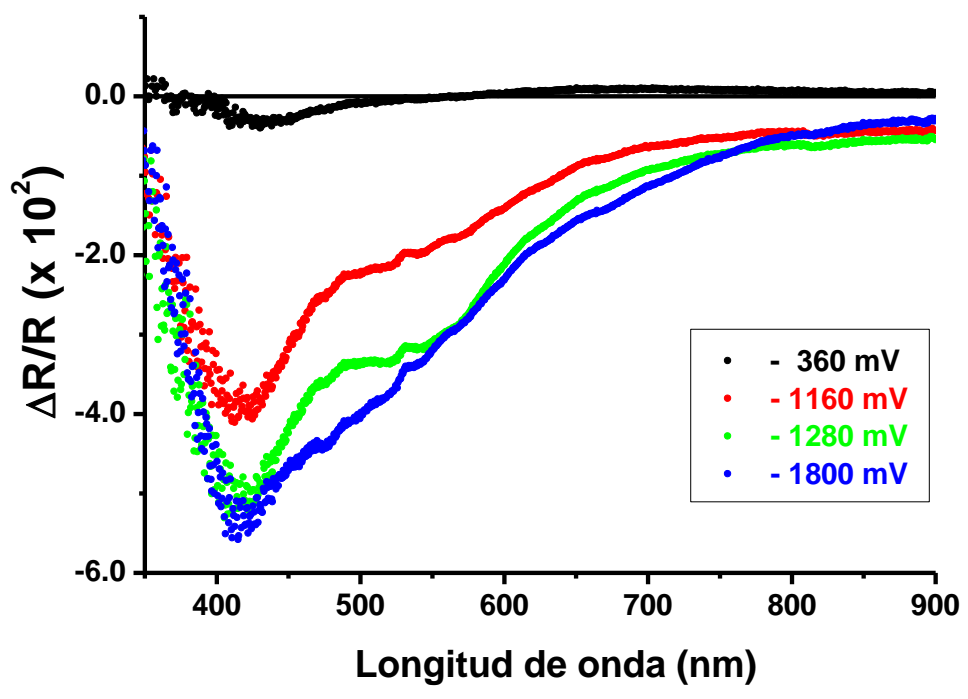

Figura 7. Espectroelectroquímica de los complejos (a) TF-5N. (b) PV-5N.

En ambos casos se utilizó: Pt / MeCN - $0.1 \mathrm{MBu}_{4} N P F_{6}$. 

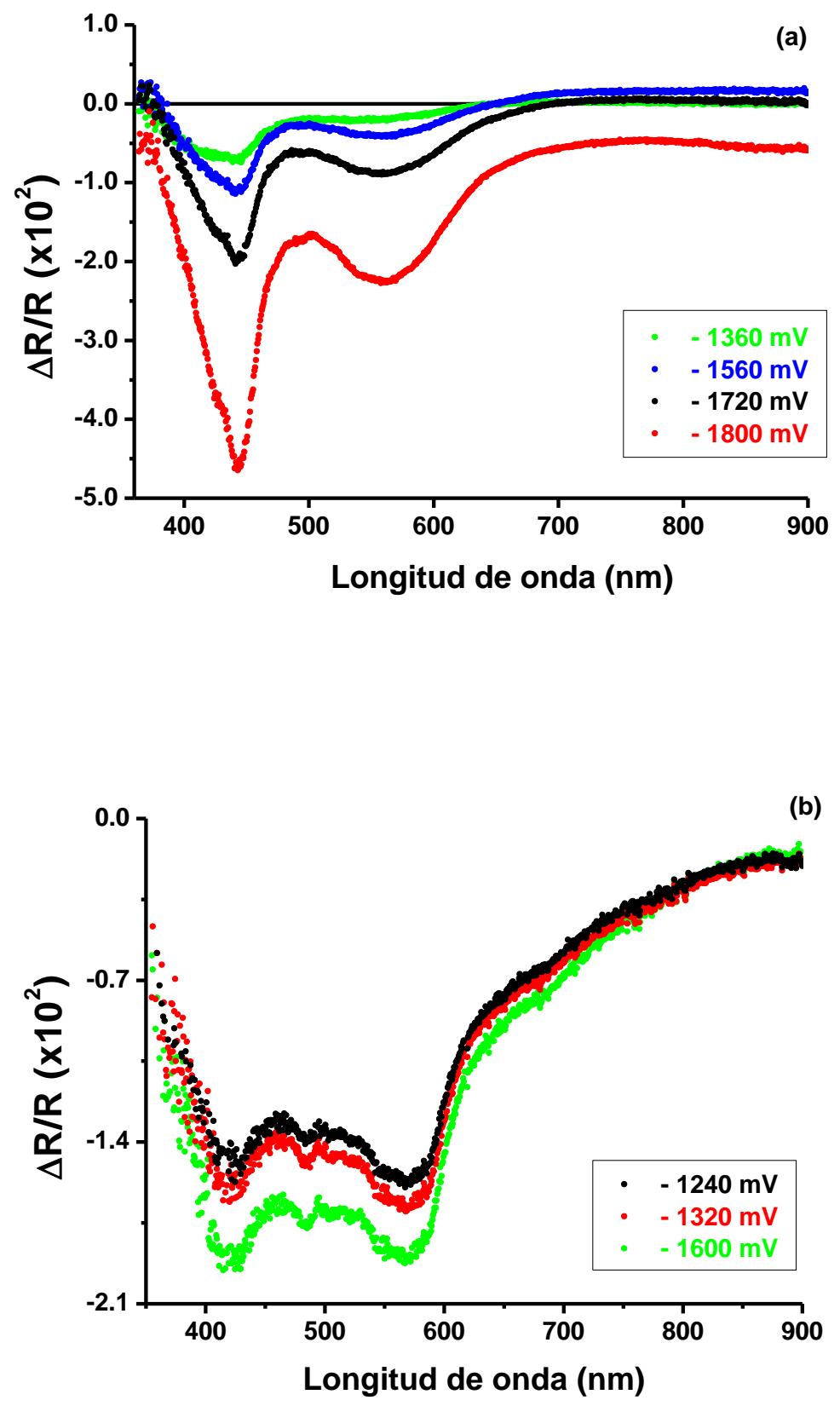

Figura 8. Espectroelectroquímica de los complejos (a) TF-TM. (b) PV-TM. En ambos casos se utilizó: Pt / MeCN - $0.1 \mathrm{M} \mathrm{Bu}_{4} N P F_{6}$. 


\subsection{Discusión}

La reducción electroquímica del grupo nitro de un compuesto aromático (Ar- $\mathrm{NO}_{2}$ ) en medios apróticos ( $\mathrm{CH}_{3} \mathrm{CN}$ y/o DMF) es por lo general, un proceso reversible monoelectrónico que conduce a la formación de un radical anión (Ar$\mathrm{NO}_{2}{ }^{*}$ ) con una estabilidad considerable (Álvarez - Griera, L. et al., 2009, Geske, D.H. et al., 1960, Mengoli, G. et al., 1970) de acuerdo a la reacción de electrodo:

$$
\mathrm{Ar}-\mathrm{NO}_{2}+\mathrm{e}^{-} \rightleftarrows \mathrm{Ar}-\mathrm{NO}_{2}{ }^{\cdot-}
$$

La primera onda de reducción de $T F-5 N$, es similar a la del $\mathrm{ClRe}(\mathrm{CO})_{3}\left(\mathrm{NO}_{2}-\right.$ phen), y es ca. $0.7 \mathrm{~V}$ más positiva que las del $\mathrm{ClRe}^{\prime}(\mathrm{CO})_{3}($ phen $)$ y $\left[\operatorname{Re}^{\prime}(\mathrm{CO})_{3} \text { (phen) }\left(\mathrm{CH}_{3} \mathrm{CN}\right)\right]^{+}$(ver tabla 1). Este cambio significativo en el potencial de reducción indica una fuerte localización de la densidad extra de electrones en el grupo $\mathrm{NO}_{2}$ (Gabrielsson, $A$. et al., 2005). Las tres reducciones siguientes 1.087, -1.514 y -1.716 V se localizan en la porción phen del ligando $\left(\mathrm{NO}_{2}-\right.$ phen) (Tokel-Takvoryan, N. E. et al., 1973, Bracco, L.L.B., Muñoz Zúniga, J. et al., 2011). Las tres ondas de reducción cuasi reversibles en $\left[\mathrm{Re}^{\prime}(\mathrm{CO})_{3}(\mathrm{tmphen})\left(\mathrm{CH}_{3} \mathrm{CN}\right)\right]^{+}$(TF-TM) aparecen a potenciales más negativos que las de $\mathrm{ClRe}^{\prime}(\mathrm{CO})_{3}$ (phen) y $\left[\operatorname{Re}^{\prime}(\mathrm{CO})_{3}(\text { phen })\left(\mathrm{CH}_{3} \mathrm{CN}\right)\right]^{+}$(tabla 1). En relación con estos dos últimos complejos (TF-TM y TF-5M), el efecto de los sustituyentes metilo unidos al ligando fenantrolina siguen el concepto de Hammett (Dewar, M. J. S. et al., 1980). Los grupos metilo, que son sustituyentes débiles donantes de electrones, cambian los potenciales de reducción de un electrón en dirección negativa. Por otro lado, es probable que el grupo nitro reducido tenga el efecto contrario ya que el segundo proceso de reducción en $\left[\mathrm{Re}^{\prime}(\mathrm{CO})_{3}\left(\mathrm{NO}_{2^{-}}\right.\right.$ phen) $\left.\left(\mathrm{CH}_{3} \mathrm{CN}\right)\right]^{+}(T F-5 N)$ (correspondiente a la primera reducción en la parte phen 
del ligando $\mathrm{NO}_{2}$-phen) se encuentra a potenciales menos negativos que para $\mathrm{CIRe}^{\prime}(\mathrm{CO})_{3}($ phen $)$ y $\left[\operatorname{Re}^{\prime}(\mathrm{CO})_{3}(\text { phen })\left(\mathrm{CH}_{3} \mathrm{CN}\right)\right]^{+}$(tabla 1$)$.

Los picos de reducción en los polímeros $\boldsymbol{P} \boldsymbol{V}-\mathbf{5 N}$ y $\boldsymbol{P} \boldsymbol{V}-\mathbf{T M}$, en ambos casos procesos irreversibles, aparecen, en general, a potenciales ligeramente menos negativos que para $\left[\operatorname{Re}^{\prime}(\mathrm{CO})_{3}\left(\mathrm{NO}_{2}-\text { phen }\right)\left(\mathrm{CH}_{3} \mathrm{CN}\right)\right]^{+} \quad(T F-5 N)$ y $\left[\operatorname{Re}^{\prime}(\mathrm{CO})_{3}(\text { tmphen })\left(\mathrm{CH}_{3} \mathrm{CN}\right)\right]^{+}$(TF-TM), respectivamente. El campo eléctrico positivo ejercido por las ca. 200 cargas positivas presentes en el polímero, puede explicar el aumento en la tendencia de reducción de los cromóforos coordinados $\left[\operatorname{Re}^{\prime}(\mathrm{CO})_{3}\left(\mathrm{NO}_{2} \text {-phen }\right)\right]^{+}$y $-\left[\operatorname{Re}^{\prime}(\mathrm{CO})_{3}(\text { tmphen })\right]^{+}$en polímeros $\left(\boldsymbol{P V}-5 \mathbf{N} \mathbf{N O}_{2^{-}}\right.$ P4VPy y (PV-TM) TM-P4VPy en relación con los de los complejos $\left[\operatorname{Re}^{\prime}(\mathrm{CO})_{3}\left(\mathrm{NO}_{2^{-}}\right.\right.$ phen $\left.)\left(\mathrm{CH}_{3} \mathrm{CN}\right)\right]^{+} \quad\left(\right.$ TF-5N) y $\quad\left[\operatorname{Re}^{\prime}(\mathrm{CO})_{3}(\text { tmphen })\left(\mathrm{CH}_{3} \mathrm{CN}\right)\right]^{+} \quad($ TF-TM $)$, respectivamente. El hecho de que los procesos de reducción en los polímeros sean irreversibles puede estar indicando que se producen reacciones químicas entre el barrido catódico y anódico que involucran el esqueleto de la cadena polimérica. Por ejemplo, la carga electrónica puede ser transferida del ligando azina inicialmente reducido a grupos vecinos a través de separadores piridina no coordinados.

Para TF-5N, en la figura 7a, las bandas observadas a partir de $-0.5 \mathrm{~V}$ en 410 $\mathrm{nm}$ (aguda) y $535 \mathrm{~nm}$ (ancha), se originan en la reducción del grupo nitro, es decir, en la formación de $\left[\operatorname{Re}^{\prime}(\mathrm{CO})_{3}\left(\mathrm{NO}_{2}{ }^{--}\right.\right.$-phen $\left.)\left(\mathrm{CH}_{3} \mathrm{CN}\right)\right]$. Este espectro es similar al del radical anión nitrobenceno (Kemula, W. et al., 1963, Núñez-Vergara, L.J. et al., 2000). A medida que el potencial ingresa a valores más negativos (a partir de $-1.0 \mathrm{~V}$ ), es decir, en la región en donde se reduce la porción phen del ligando $\mathrm{NO}_{2}$ - phen, se define claramente una nueva banda a $535 \mathrm{~nm}$ que indica que el complejo $\left[\operatorname{Re}^{\prime}(\mathrm{CO})_{3}\left(\mathrm{NO}_{2}{ }^{\bullet-}\right.\right.$-phen $)\left(\mathrm{CH}_{3} \mathrm{CN}\right)$ ] experimenta una reducción adicional, para dar $\left[\operatorname{Re}^{\prime}(\mathrm{CO})_{3}\left(\mathrm{NO}_{2}{ }^{\circ-}\right)\left(\text { phen }{ }^{\circ}\right)\left(\mathrm{CH}_{3} \mathrm{CN}\right)\right]^{-}$, es decir, el anión como producto de la segunda reducción. A potenciales más negativos tienen lugar reducciones adicionales como indica el ACV correspondiente, las cuales sólo se ven reflejadas en el aumento de la intensidad de la banda a $535 \mathrm{~nm}$ respecto de 
la de $410 \mathrm{~nm}$. Otras características espectrales no parecen ser alteradas por la posterior entrada de tres electrones en (phen $\left.{ }^{*}\right)$.

Los cambios espectrales debido a la reducción electrónica del polímero $\mathbf{P V - 5 N}$ son similares a los observados para $T F-5 N$ en la figura $7 \mathrm{a}$, aunque menos definidos (figura $7 \mathrm{~b}$ ). Las bandas de $\boldsymbol{P V}-\mathbf{5 N}$ observadas alrededor de $410 \mathrm{~nm}$, a partir de -0.3 V, provienen de $\left\{(\mathrm{vpy})_{2} \mathrm{vpy}\left[\operatorname{Re}^{\prime}(\mathrm{CO})_{3}\left(\mathrm{NO}_{2}{ }^{\circ-}-\text { phen }\right)\right]_{\mathrm{n} \sim 200}\right.$. Sin embargo, el polímero $\mathbf{P V}-\mathbf{5 N}$ consta de cromóforos - $\left[\mathrm{Re}^{\prime}(\mathrm{CO})_{3}\left(\mathrm{NO}_{2} \text {-phen) }\right]^{+}\right.$distribuidos al azar a través de la coordinación de piridinas al esqueleto de la poli-4-vinilpiridina. A nivel local, podría haber regiones sin espaciador piridina, o con uno o más separadores, etc. Debido a que entre los centros de $\mathrm{Re}^{l}$ han sido estimadas distancias tan cortas como $8 \AA$ (Bracco, L.L.B. et al., 2008) (figura 9), existe una distribución de los grupos metálicos en espacios reducidos de la cadena polímerica. Por lo tanto, $\mathbf{P V - 5 N}\left\{(\mathrm{vpy})_{2} \mathrm{Vpy}\left[\mathrm{Re}^{\prime}(\mathrm{CO})_{3}\left(\mathrm{NO}_{2}{ }^{*}-\text { phen }\right)\right\}_{n \sim 200}\right.$ es solamente una representación promedio de las diferentes distribuciones espaciales de los grupos $\mathrm{NO}_{2}{ }^{\circ}$-phen reducidos en el polímero. Como resultado, diferentes cromóforos $\left[\operatorname{Re}^{\prime}(\mathrm{CO})_{3}\left(\mathrm{NO}_{2}{ }^{*}\right.\right.$-phen $\left.)\right]$ en diversas configuraciones espaciales están contribuyendo a las características espectrales de la figura $7 b$, generando un ensanchamiento de las bandas de absorción. Nuevas reducciones a potenciales más negativos producen $\left\{(\mathrm{vpy})_{2} \mathrm{Vpy}\left[\operatorname{Re}^{\prime}(\mathrm{CO})_{3}\left(\mathrm{NO}_{2}{ }^{\circ-}{ }^{-p h e n}{ }^{\circ}\right)\right]\right\}_{n \sim 200}$ y reducciones subsecuentes en los ligandos phen**.

Una comparación de las figuras $8 \mathrm{a}$ y $8 \mathrm{~b}$ muestra que el espectro resultante de las especies reducidas de TF-TM es significativamente diferente del de las especies reducidas que se producen a partir del polímero $\mathbf{P V}$-TM. Por ejemplo, la relación $(\Delta R / R) 440 \mathrm{~nm} /(\Delta R / R) 565 \mathrm{~nm}$ es cercana a 2 en la reducción de TF-TM, mientras que $(\Delta R / R) 440 \mathrm{~nm} /(\Delta R / R) 565 \mathrm{~nm}$ es $\sim 1$ en la reducción de $\boldsymbol{P V}$ - $\boldsymbol{T M}$. Es probable que las especies reducidas principales que contribuyen a las características espectrales de las figuras $8 \mathrm{a}$ y $8 \mathrm{~b}$ sean $\left[\mathrm{Re}^{\prime}(\mathrm{CO})_{3}\left(\mathrm{tmphenH}^{*}\right)\left(\mathrm{CH}_{3} \mathrm{CN}\right)\right]^{+} \quad($ TF-TM $) \quad$ y $\left\{(\mathrm{vpy})_{2} \mathrm{vpy}\left[\mathrm{Re}^{\prime}(\mathrm{CO})_{3}\left(\text { tmphen }^{*}\right)\right]\right\}_{n \sim 200}$ $(\boldsymbol{P V}$ - $T M)$ respectivamente. 


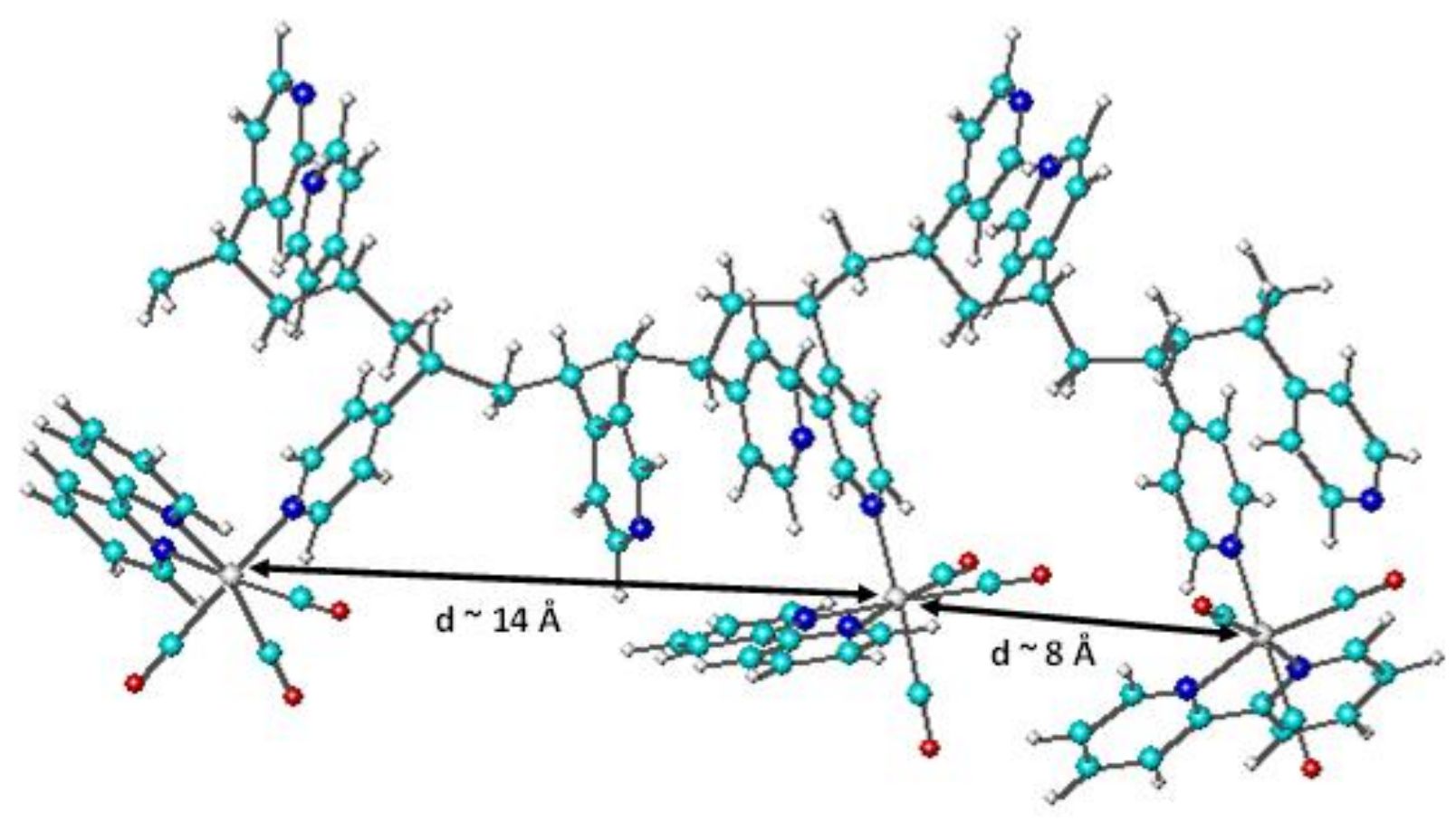

Figura 9. Representación de un fragmento (solamente 3 cromóforos de $R e^{\prime}$ ) de el polímero $\left\{(\mathrm{vpy})_{2}-\mathrm{vpy}\left[\mathrm{Re}^{\prime}(\mathrm{CO})_{3}(\mathrm{bpy})\right]\right\}_{n \sim 200}\left(\mathrm{CF}_{3} \mathrm{SO}_{3}\right)_{n \sim 200}$ mostrando la distancia promedio entre cromóforos. 


\subsection{Conclusiones}

Los radicales aniones $\left[\operatorname{Re}^{\prime}(\mathrm{CO})_{3}\left(\mathrm{NO}_{2}{ }^{*-}\right.\right.$ - phen $\left.)\right]$ y $\left[\operatorname{Re}^{\prime}(\mathrm{CO})_{3}\left(\right.\right.$ tmphen $\left.\left.{ }^{*}\right)\right]$, generados en la electroreducción de los complejos TF-5N y TF-TM respectivamente fueron caracterizados espectroelectroquímicamente.

Los radicales aniones $\left\{(\mathrm{vpy})_{2} \mathrm{vpy}\left[\mathrm{Re}^{\prime}(\mathrm{CO})_{3}\left(\mathrm{NO}_{2}{ }^{--}\right.\right.\right.$-phen $\left.\left.)\right]\right\}$y $\left\{(\mathrm{vpy})_{2} \mathrm{vpy}\left[\mathrm{Re}^{\prime}\right.\right.$ $(\mathrm{CO})_{3}\left(\right.$ tmphen $\left.\left.\left.^{*}\right)\right]\right\}$ generados en la electroreducción de polímeros de poli-4vinilpiridina, $\boldsymbol{P V}-\mathbf{5 N}$ y $\boldsymbol{P V}-\mathbf{T M}$ respectivamente fueron caracterizados espectroelectroquímicamente.

Siguiendo la reacción directa entre el $\mathrm{e}_{\text {solv }}^{-}$y el polímero $\mathbf{P V - 5 N}$ por radiólisis de pulsos (Bracco, L.L.B, et al., 2011) para generar el anión radical $\left\{(\mathrm{vpy})_{2} \mathrm{Vpy}\right.$ $\left[\operatorname{Re}^{\prime}(\mathrm{CO})_{3}\left(\mathrm{NO}_{2}{ }^{-}\right.\right.$-phen) $\left.]\right\}$, se ha encontrado coincidencia en el espectro de absorción con el radical reducido obtenido espectroelectroquímicamente a partir del mismo complejo polimérico. Mostrando en ambos casos, la absorción de la especie reducida aproximadamente a $410 \mathrm{~nm}$ como se puede ver en la figura 10 . 

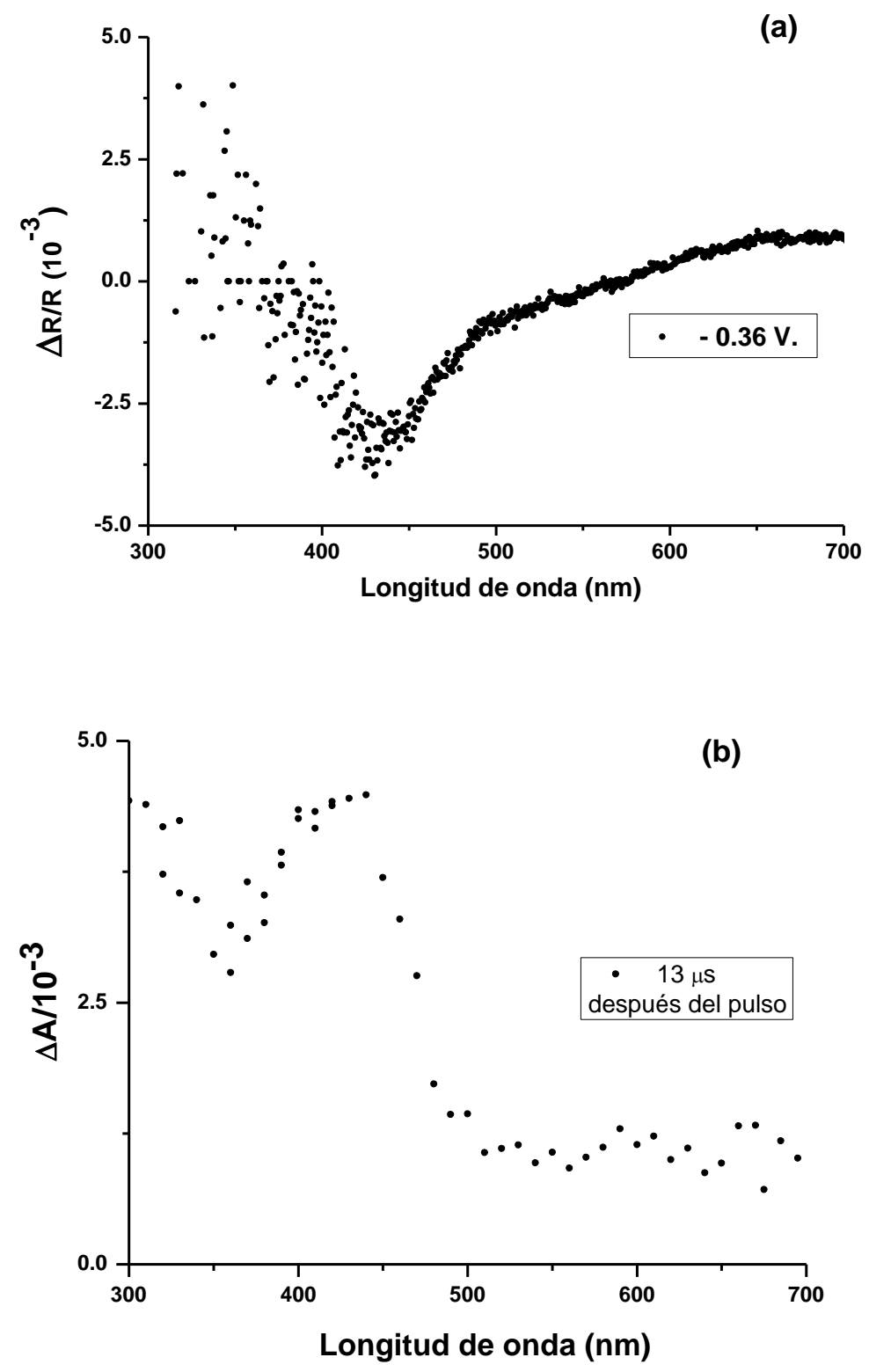

Figura 10. (a). Espectroelectroquímica de PV-5N para $-0.36 \mathrm{~V}$ (de la figura 7). (b) Espectro en presencia de $N_{2}$, reacción directa del $e_{\text {solv }}^{-}$y $\boldsymbol{P V}-5 \mathrm{~N}$ mediante radiólisis de pulso (Bracco, L.L.B. et al., 2011). 


\subsection{Referencias}

Álvarez - Griera, L.; Gallardo, I.; Guirado, G. Electrochim. Acta 2009, 54, $5098-5108$.

Bracco, L.L.B.; Juliarena, M.P.; Ruiz, G.T.; Féliz, M.R.; Ferraudi, G.J. y Wolcan, E. J. Phys. Chem. B. 2008, 112, 11506 - 11516.

Bracco, L.L.B.; Einschlag, F.S.G.; Wolcan, E. y Ferraudi, G. J., J. Photochem. Photobiol., A 2009, 208, 50 - 58.

Bracco, L.L.B.; Féliz, M.R y Wolcan, E. Journal of Photochemistry and Photobiology A: Chemistry. 2010, 210, 23 - 30.

Bracco, L.L.B.; Lezna, R. O.; Muñoz Zuñiga, J.; Ruiz, G. T.; Féliz, M. R.; Ferraudi, G. J.; García Einschlag, F. S. y Wolcan, E. Inorg. Chim. Acta. 2011, 370, 482 - 491.

Costamagna, J.; Ferraudi, G.; Canales, J.; Vargas, J. Coord. Chem. Rev. 1996, 148, 221 - 248.

Dewar, M.J.S. y Dougherty, R.C. Teoría De Las Perturbaciones De Los Orbitales Moleculares (PMO) En Química Orgánica. Reverté, S. A., Barcelona, 1980.

Fox, M.A.; Chanon, M. Photoinduced Electron Transfer; Elsevier, Amsterdam, 1988. 
Gabrielsson, A.; Matousek, P.; Towrie, M.; Hartl, F.; Zális, S. y Vlček Jr, A. J. Phys. Chem. A. 2005, 109, 6147-6153.

Geske, D.H. y Maki, A.H. J. Am. Chem. Soc. 1960, 82, 2671 - 2676.

Hawecker, J.; Lehn, J-M. y Ziessel, R. J. Chem. Soc., Chem. Commun. 1983, $536-538$.

Higgins, B.; DeGraff, B.A. y Demas, J.N. Inorg. Chem. 2005, 44, 6662 - 6669.

Kalyanasundaram, K. Coord. Chem. Rev. 1982, 46, 159 - 244.

Kalyanasundaram, K. J. Chem. Soc., Faraday Trans. 2, 1986, 82, 2401 2415.

Kalyanasundaram, K. y Grätzel, M. Photosensitization and Photocatalysis Using Inorganic and Organometallic Compounds, Kluwer Academic Publishers, Dordrecht, 1993.

Kemula, W. y Sioda, R. Nature. 1963, 197, 587 - 588.

Luong, J.C.; Nadjo, L. y Wrighton, M.S. J. Am. Chem. Soc. 1978, 100, 5790 5795.

Mengoli, G. y Vidotto, G. Makromol. Chem. 1970, 133, 279 - 288.

Núñez-Vergara, L.J.; Sturm, J.C.; Olea-Azar, C.; Navarrete-Encina, P.; Bollo, S. y Squella, J.A. Free Radical Res. 2000, 32 (5), 399 - 409.

Paolucci, F.; Marcaccio, M.; Paradisi, C.; Roffia, S.; Bignozzi, C.A. y Amatore, C. J.Phys. Chem. B. 1998,102, 4759 - 4769. 
Pavlishchuk, V.V. y Addison, A.W. Inorg. Chim. Acta 2000, 298, 97-102.

Sacksteder, L.; Lee, M.; Demas, J.N. y DeGraff, B.A. J. Am. Chem. Soc. 1993, $1158230-8238$.

Stufkens, D.J. y Vlček Jr, A. Coord. Chem. Rev. 1998, 177,127-179.

Tokel-Takvoryan, N. E.; Hemingway, R. E. y Bard, A. J. J. Am. Chem. Soc. 1973, 95 (20), 6582 - 6589.

Vogler, A. y Kunkely, H. Coord. Chem. Rev. 2000, 200-202, 991 - 1008.

Wolcan, E. y Ferraudi, G. J. Phys. Chem. A, 2000, 104 (41), 9281 - 9286.

Wolcan, E. y Féliz, M.R. Photochem. Photobiol. Sci. 2003, 2, 412 - 417.

Wolcan, E.; Alessandrini, J.L. y Féliz, M.R. J. Phys. Chem. B. 2005,109, $22890-22898$.

Yam, V.W-W.; Wong, K.M-C.; Lee, V.W.-M.; Lo, K.K.-W. y Cheung, K.-K. Organometallics 1995, 14, 4034 - 4036.

Yoon, D.I.; Berg-Brennan, C.A.; Lu, H. y Hupp, J.T. Inorg. Chem. 1992, 31, 3192 - 3194. 


\section{Trabajos publicados y}

presentaciones a congresos en el marco de la tesis. 
Trabajos publicados y presentaciones a congresos en el marco de la tesis. | ii 
Trabajos publicados y presentaciones a congresos en el marco del trabajo de tesis.

\section{- Participación en Congresos y Jornadas.}

"Estudio Electroquímico de Fotocatalizadores Multielectrónicos." Muñoz Zúñiga J. Jornada de becarios del INIFTA. La Plata, 10 - 14 de Octubre de 2008.

"Estudio Electroquímico de Fotocatalizadores Multielectrónicos para la Generación de Hidrógeno Solar" Muñoz Zúñiga J., Lezna, R.O., Tacconi, N. R. y MacDonnell, F. M. XVI Congreso Argentino de Fisicoquímica y Química Inorgánica. Universidad Nacional de Salta - Argentina. 2009.

"Electroquímica de Fotocatalizadores para la Generación de Hidrógeno Solar" Muñoz Zúñiga, J. Jornada de becarios del INIFTA, La Plata 13 - 16 de Octubre de 2009.

"Estudio Espectroelectroquímico del Fotocatalizador Mono- BentP para la Generación de Hidrógeno Solar". Muñoz Zúñiga, J. Jornada de becarios del INIFTA, La Plata 18 - 20 de Octubre de 2010. 
"Espectroelectroquímica de los Radicales Aniones Generados en la Electroreducción de - $\left[\operatorname{Re}^{\prime}(\mathrm{CO})_{3}(5-N \text { itro-1,10-fenantrolina })\right]^{+}$y $-\left[\operatorname{Re}^{\prime}(\mathrm{CO})_{3}(3,4,7,8\right.$ tetrametil-1,10-fenantrolina) $]^{+}$en Polímeros de poli-4-vinilpiridina.". Bracco, L. L. B.; Muñoz Zúñiga, J.; Wolcan, E.; Féliz, M. R. y Lezna, R. O. XVII Congreso Argentino de Fisicoquímica y Química Inorgánica. Universidad Nacional de Córdoba - Argentina. 2011.

\section{- Trabajos publicados en revistas periódicas.}

"Spectroelectrochemical Detection of the Multi-Electron Reduction and Protonation of the tatpp Ligand in the [(bpy) ${ }_{2} R u($ tatpp) $] \mathrm{Cl}_{2}$ Complex ". Lezna, R. O. ; Tacconi, N. R.; Muñoz Zúñiga, J. and MacDonnell F. M. Journal of the Argentine Chemical Society, 2009, 97, (1), 273 - 288.

"Photochemical Two-Electron Reduction of a Dinuclear Ruthenium Complex Containing a Bent Tetraazatetrapyridopentacene Bridging Ligand: Pushing Up the LUMO for Storing More Energy" . Shreeyukta, S.; de Tacconi, N.R.; Diaz, N.R.G.; Lezna, R. O.; Muñoz Zuñiga, J.; Abayan, K. y MacDonnell, F.M. Inorganic Chemistry, 2011, 50, (19), 9318 - 9328.

"On the mechanism of formation and spectral properties of radical anions generated by the reduction of $-\left[\operatorname{Re}^{\prime}(C O)_{3}(5 \text {-nitro-1,10-phenanthroline })\right]^{+}$and $\left[\operatorname{Re}^{\prime}(\mathrm{CO})_{3}(3,4,7,8 \text {-tetramethyl-1,10-phenanthroline })\right]^{+}$pendants in poly-4vinylpyridine polymers" Bracco, L. L.B.; Lezna, R. O.; Muñoz Zuñiga, J.; Ruiz, G. T.; Féliz, M. R.; Ferraudi, G. J.; García Einschlag, F. S. y Wolcan, E. Inorganica Chimica Acta, 2011, 370, 482 - 491. 